\title{
Developing high performance and stable hetero-structured cathodes and fundamental understanding of oxygen reduction and reaction behavior
}

Xinxin Zhang

Follow this and additional works at: https://researchrepository.wvu.edu/etd

\section{Recommended Citation}

Zhang, Xinxin, "Developing high performance and stable hetero-structured cathodes and fundamental understanding of oxygen reduction and reaction behavior" (2016). Graduate Theses, Dissertations, and Problem Reports. 7039.

https://researchrepository.wvu.edu/etd/7039

This Dissertation is protected by copyright and/or related rights. It has been brought to you by the The Research Repository @ WVU with permission from the rights-holder(s). You are free to use this Dissertation in any way that is permitted by the copyright and related rights legislation that applies to your use. For other uses you must obtain permission from the rights-holder(s) directly, unless additional rights are indicated by a Creative Commons license in the record and/ or on the work itself. This Dissertation has been accepted for inclusion in WVU Graduate Theses, Dissertations, and Problem Reports collection by an authorized administrator of The Research Repository @ WVU.

For more information, please contact researchrepository@mail.wvu.edu. 


\title{
DEVELOPING HIGH PERFORMANCE AND STABLE HETERO- STRUCTURED CATHODES AND FUNDAMENTAL UNDERSTANDING OF OXYGEN REDUCTION AND REACTION BEHAVIOR
}

\author{
Xinxin Zhang
}

\author{
Dissertation submitted to the \\ Benjamin M. Statler College of Engineering and Mineral Resources \\ at West Virginia University \\ in partial fulfillment of the requirements \\ for the degree of
}

Doctor of Philosophy

in

Mechanical Engineering

Department of Mechanical and Aerospace Engineering

Xingbo Liu, Ph.D., Chair

Harry W. Abernathy, Ph.D.

Ismail Celik, Ph.D.

Xueyan Song, Ph.D.

Edward Sabolsky, Ph.D.

Morgantown, West Virginia

2016

Keywords: Solid Oxide Fuel Cells; Hetero-structured Cathode Materials; Electrochemical Performance; Long-term Stability; Kinetic Driving Force Copyright 2016 Xinxin Zhang 


\section{Abstract \\ Developing High Performance and Stable Hetero-structured Cathodes and Fundamental Understanding of Oxygen Reduction and Reaction Behavior}

\section{ByXinxin Zhang}

New hetero-structured $\mathrm{La}_{2} \mathrm{NiO}_{4+\delta}(\mathrm{LNO}) / \quad\left(\mathrm{La}_{0.6} \mathrm{Sr}_{0.4}\right)_{0.95} \mathrm{Co}_{0.2} \mathrm{Fe}_{0.8} \mathrm{O}_{3-\delta} \quad(\mathrm{LSCF}) \quad \mathrm{SOFC}$ cathodes have been successfully fabricated by the infiltration technique for long-term stability and oxygen reduction reaction (ORR) kinetic consideration. The current comparative study of LSCF and LNO-infiltrated LSCF shows a significant reduction in polarization resistance by one order of magnitude and a $67 \%$ increase in maximum power density by using LNO-infiltrated LSCF cathodes at $750^{\circ} \mathrm{C}$ in air. The electronic conductivity relaxation (ECR) results demonstrate that the remarkable improved oxygen exchange properties of LNO/LSCF hetero-structured cathodes are attributed to not only the increase of surface reaction area, but also the introduction of LNO surface and LNO-LSCF interface with rapid oxygen exchange behavior. A trace amount of $\mathrm{Co}, \mathrm{Sr}$ and more oxygen defects stabilized in the LNO layer have been identified by TEM, EDXS, XPS, XRD and iodine titration experiments, giving rise to the enhancement of stability and surface catalytic activity. A low degradation rate of $0.39 \%$ at a constant current density of 250 $\mathrm{mA} \cdot \mathrm{cm}^{-2}$ can be still achieved for the fuel cell using LNO-infiltrated LSCF cathodes after long-term durability of about $500 \mathrm{~h}$ at $750^{\circ} \mathrm{C}$, accompanied by nanoparticles growth/aggregation, delamination and slight lanthanum enrichment on LNO surfaces. The benefits from the presence of the LNO nanoparticles demonstrate that LNO-infiltrated LSCF materials can act as high active surface oxygen exchange cathodes with promising fast ORR behavior and stability.

To further enhance the long-term stability of infiltrated $\left(\mathrm{La}_{0.6} \mathrm{Sr}_{0.4}\right)_{0.95} \mathrm{Co}_{0.2} \mathrm{Fe}_{0.8} \mathrm{O}_{3-\delta}$ (LSCF) cathode, two kinds of new hetero-structured cathodes, $\mathrm{CeO}_{2} \& \mathrm{LNO}$ co-infiltrated LSCF and $\mathrm{La}_{2-x} \mathrm{NiO}_{4+\delta}$-infiltrated $\mathrm{LSCF}$, have been designed. $\mathrm{La}_{2} \mathrm{NiO}_{4+\delta}(\mathrm{LNO})$, as an superior performance promoter, and $\mathrm{CeO}_{2}$, as a particle growth retardant due to the high melting point and good wettability, were co-infiltrated into the LSCF backbone to mitigate the delamination and growth/aggregation of in-situ formed nano-sized infiltrants. A more advantageous microstructure was confirmed in the co-infiltrated sample relative to those 
infiltrated with LNO alone. The original morphology was largely retained in the coinfiltrated sample after heat treatment, showing finer surface particles, better connection between the infiltrant network and the LSCF backbone. XPS and EIS results reveal that the substitution of stoichiometric $\mathrm{La}_{2} \mathrm{NiO}_{4+\delta}$ by A-site deficient $\mathrm{La}_{2-x} \mathrm{NiO}_{4+\delta}$ can effectively suppress lanthanum enrichment and enhance the stability after cathodic polarization treatment observed by measurement. The advantages of both modified hetero-structured cathodes suggest that $\mathrm{CeO}_{2} \& \mathrm{La}_{2-x} \mathrm{NiO}_{4+\delta}$ co-infiltrated LSCF is a promising heterostructured cathode to mitigate the LSCF electrode degradation. 


\section{ACKNOWLEDGMENTS}

My deepest gratitude goes first and foremost to my advisor Dr. Xingbo Liu for his continuous support of my Ph.D's study and research, for his patience, enthusiasm, inspiration, and expertise. I am also extremely grateful to Dr. Ismail Celik, Dr. Harry W. Abernathy, Dr. Xueyan Song and Dr. Edward Sabolsky for their mentoring and assistance, as well as being my committee members.

We acknowledge use of the WVU Shared Research Facilities. Thanks for Dr. Weiqiang Ding, Dr. Marcela Redigolo, and Harley Hart for their skills and help in XPS, SEM/EDS, XRD analysis.

Thanks all my group members, Dr. Wenyuan Li, Dr. Greg Collins, Bo Guan, Dr. Youkun Tao, Yue Zhou, Shanshan Hu, Nan Zhang and He Qi, for their assistance in experimental and discussion. I also want to thank Tao Yang for his generous and knowledgeable suggestions. I appreciate that I can get help from all of them and I am very happy we can have a memorable happy time.

Last but not the least; I would like to thank my parents for giving birth to me at the first place and supporting me spiritually throughout my life. 


\section{Table of Contents}

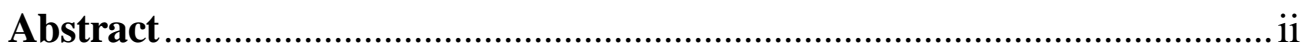

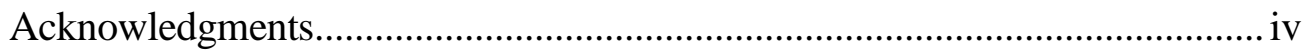

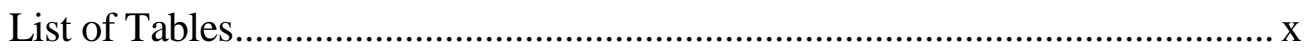

Chapter 1: Introduction and Research Objectives ........................................ 1

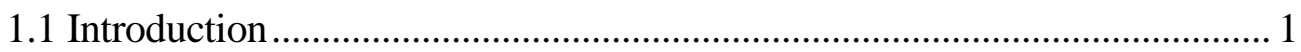

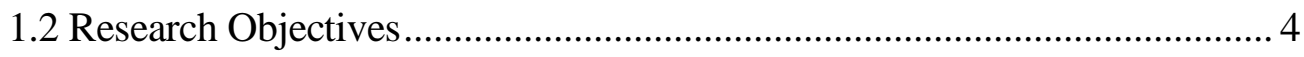

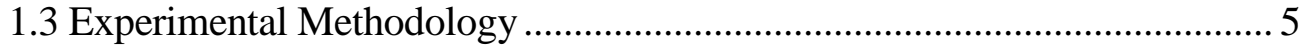

1.3.2Dense Hetero-Structured Cathode Layers Fabrication ........................ 6

1.3.3Symmetrical Cells and Fuel Cells Assembling.................................. 6

1.3.4 Oxygen Reduction Reaction Activity Measurements........................ 7

1.3.5 Ex situ Characterization................................................................... 9

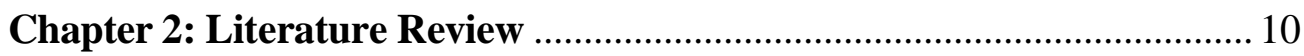

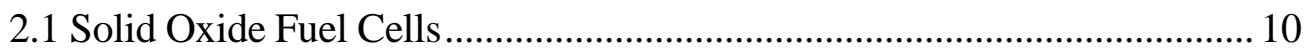

2.1.1 Introduction and Mechanism........................................................... 10

2.1.2 Component Requirements .......................................................... 14

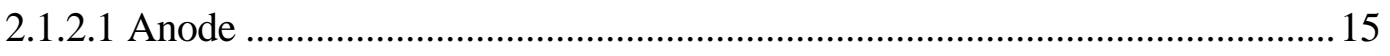

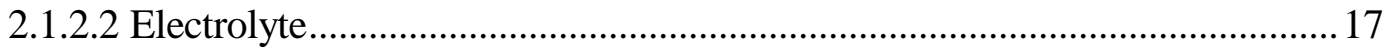

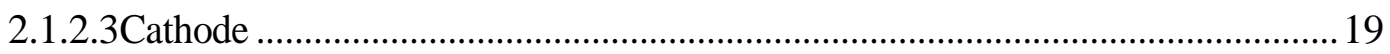

2.2 Fundamental Background of Solid Oxide Fuel Cells Cathode................... 21

2.2.1Cathode Oxygen Reduction Reaction Mechanism Models............... 21

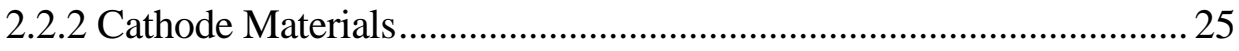

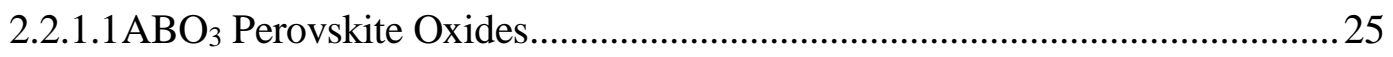

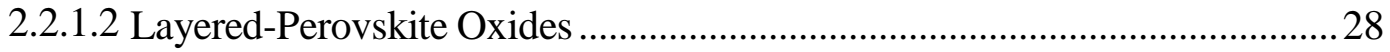

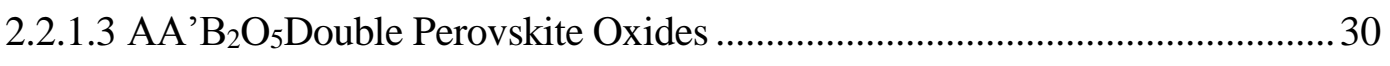

2.2.3 Long-term Stability of Sr-doped Perovskite Cathode....................... 32

2.2.4 Hetero-Structured Cathode ............................................................... 34

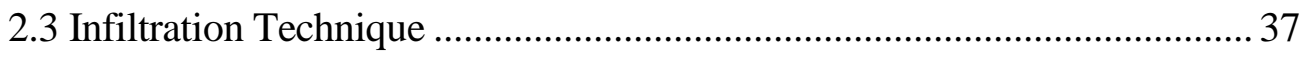

Chapter 3: $\mathrm{La}_{2} \mathrm{NiO}_{4+\delta}$-Infiltrated (La0.6Sr0.4) $)_{0.95} \mathrm{Co}_{0.2} \mathrm{Fe}_{0.8} \mathrm{O}_{3-\delta}$ Hetero-structure

Cathode 40 


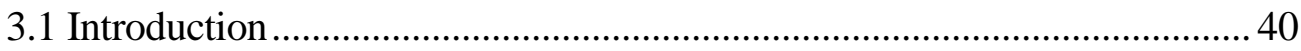

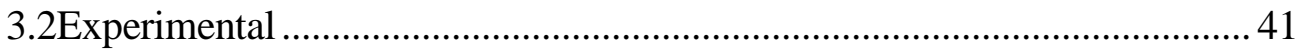

3.3Results and Discussion .............................................................................. 43

3.3.1 Phase Composition ........................................................................ 43

3.3.2 Infiltration techniques........................................................................ 44

3.3.3Electrochemical Performance of Infiltrated Cathode............................ 48

3.3.3 Surface and Interface Oxygen Exchange Kinetics Behavior.............. 52

3.3.5 Surface and Bulk Elements Distribution and Crystal Structure of LNO Layers 55

3.3.6 Long-term stability and Degradation mechanisms .............................. 64

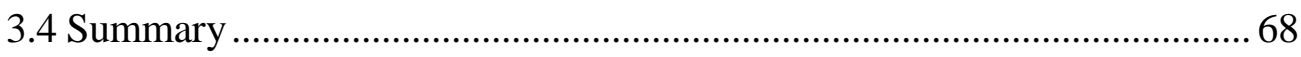

Chapter 4: Effect of A-site Deficient $\mathrm{La}_{2} \mathrm{NiO}_{4+\delta}$ on Stability of Hetero-structured

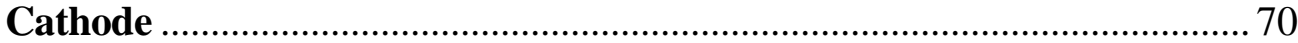

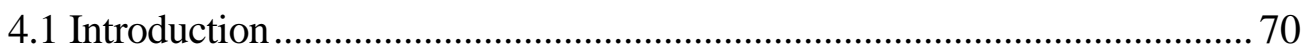

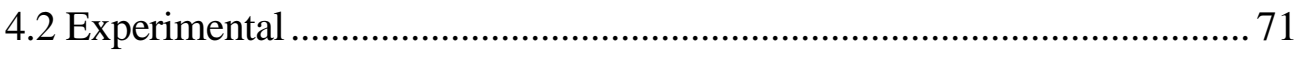

4.2 Results and Discussion ............................................................................ 73

4.2.1 Phase Composition and Structure ………………………………….... 73

4.2.2 Conductivity, Oxygen Non-Stoichimetry and Electrochemical Performance77

4.2.3 Infiltrated Hetero-structure A-site deficient LNO-LSCF Cathode ... 82

4.3 Summary 85

Chapter 5: $\mathrm{CeO}_{2} \& \mathrm{La}_{2} \mathrm{NiO}_{4+\delta} \mathrm{Co}$-Infiltrated $\left(\mathrm{La}_{0.65} \mathrm{Sr}_{0.4}\right)_{0.95} \mathrm{Co}_{0.22} \mathrm{Fe}_{0.8} \mathrm{O}_{3-\delta}$ Cathode87

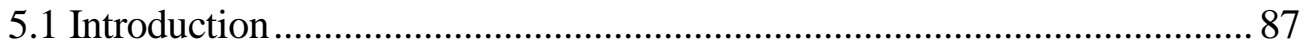

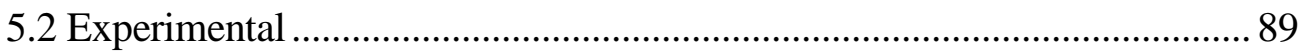

5.3 Results and Discussion .......................................................................... 91

5.3.1 X-ray Diffraction Pattern for phase composition................................. 91

5.3.2 Effect of microstructure before and after long-term stability .............93

5.3.3 Electrochemical Performance ........................................................... 95

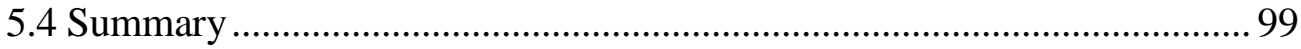

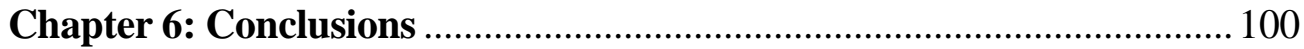

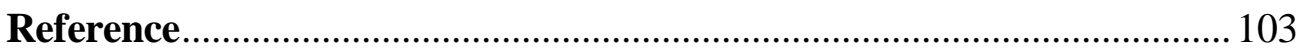




\section{List of Figures}

Figure 2.1 Schematic of an electrolyte supported single SOFC cells ......................................11

Figure 2.2 Structure of simple cubic perovskite..................................................................26

Figure 2.3 Structure of layered perovskite …………………..........................................29

Figure 2.4 Structure of double-layered perovskite …………………………………….......

Figure 3.1 XRD patterns of (a) bare LSCF cathode and (b) LNO-infiltrated LSCF on

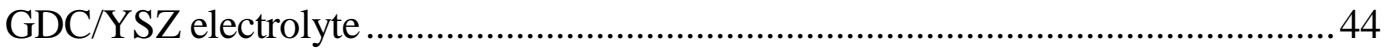

Figure 3.2 Microstructure of LNO-infiltrated LSCF electrode with different heating rate for low-temperature preprocess

Figure 3.3 Microstructure of LNO-infiltrated LSCF electrode with different mass of disperser TX100 with 1mol LNO

Figure 3.4 Microstructure of LNO-infiltrated LSCF electrode with different concentration of LNO infiltration solutions

Figure 3.5 Microstructure of LNO-infiltrated LSCF electrode with and without vacuum treatment during infiltration process

Figure 3.6 SEM image of LSCF cathode (a) without LNO infiltration or with (b) 8.60 wt. \%, (c) 15.4 wt. \% and (d) 20.3 wt. \% of LNO infiltration after $850^{\circ} \mathrm{C}$ sintering for $1 \mathrm{~h}$

Figure 3.7 Typical electrochemical impedance spectra of symmetrical cells with different LNO loading under $\mathrm{OCV}$ at $650^{\circ} \mathrm{C}$ and $700^{\circ} \mathrm{C}$

Figure 3.8 Cathode polarization resistance as function of measuring temperature for LSCF cathode with different LNO loading .48

Figure 3.9 Typical electrochemical impedance spectra of symmetrical cells using LSCF cathodes (a) without and (b) with LNO infiltration under OCV at 700 and $750^{\circ} \mathrm{C} .50$

Figure 3.10 Typical electrochemical impedance spectra of symmetrical cells using LSCF cathodes (a) without and (b) with LNO infiltration under OCV at 700 and $750^{\circ} \mathrm{C} .51$

Figure 3.11 Cell voltage and power density as function of current density for (a) pure LSCF and (b) LNO-infiltrated LSCF measured at $750^{\circ} \mathrm{C}$ during cell operation of $500 \mathrm{~h} . .52$ 
Figure 3.12 Typical ECR data and fitting results of (a) LSCF and (b) LNO-coated LSCF (LNO/LSCF) samples at $700^{\circ} \mathrm{C}$ under air conditions

Figure 3.13 Surface oxygen exchange coefficient of (a) LSCF and (b) LNO-coated LSCF $\left(\mathrm{LNO} / \mathrm{LSCF}\right.$ ) samples as a function of $\mathrm{PO}_{2}{ }^{1 / 2}$ at $700^{\circ} \mathrm{C}$

Figure 3.14 TEM images and EDAX spectra of LNO-infiltrated LSCF cathode (a) before on-cell testing and (b) after long term testing .57

Figure 3.15 Cross-section morphology of LNO-coated LSCF material...............................58

Figure 3.16 EDX line scan results for LNO-coated LSCF ...............................................58

Figure 3.17 Ni 3p, Co 2p, Fe 2p and Sr 3d XPS spectra of $\mathrm{La}_{2} \mathrm{NiO} 4-\delta$-coated LSCF surface59

Figure 3.18 Sr 3d XPS spectra of LSCF (a) fresh surface; (b) surface after polarization and of $\mathrm{La}_{2} \mathrm{NiO}_{4+\delta}$-coated LSCF (c) fresh surface and (d) surface after polarization.........60

Figure 3.19 XRD data for (a) pure LNO pellet and (b) LNO layer coated on LSCF surface recorded at room temperature. $(+)$ represent the experimental data points, solid red line is refined data fit

Figure 3.20 The crystal structure of $\mathrm{A}_{2} \mathrm{BO}_{4}(*)$ represent the interstitial oxygen atomics ...63

Figure 3.21 Cell voltage as function of time at a constant current density of $250 \mathrm{~mA} \mathrm{~cm}^{-2}$ for both bare LSCF and LNO-infiltrated LSCF. 65

Figure 3.22 SEM image of LSCF cathode with LNO infiltration (a) and (b) without longterm operation; (c) and (d) with $1000 \mathrm{~h}$ long-term heat treatment at $750^{\circ} \mathrm{C}$; (e) and (f) with $500 \mathrm{~h}$ long-term operation under a constant current density of $250 \mathrm{~mA} \cdot \mathrm{cm}^{-2} .67$

Figure 3.23 Ni 3p and La 3d XPS spectra of $\mathrm{La}_{2} \mathrm{NiO}_{4+\delta}$-coated LSCF (a) and (b) fresh surface; (c) and (d) surface after polarization .68

Figure 4.1 XRD pattern of $\mathrm{La}_{2-\mathrm{x}} \mathrm{NiO}_{4+\delta}(\mathrm{x}=0-0.2)$ powders after sintering $1000^{\circ} \mathrm{C}$ for $6 \mathrm{~h}$ in air: (a) $\mathrm{x}=0$; (b) $\mathrm{x}=0.05$; (c) $\mathrm{x}=0.10$; (d) $\mathrm{x}=0.15$; (e) $\mathrm{x}=0.2$. .74

Figure 4.2 Rietveld refinement profiles of A-site deficient $\mathrm{La}_{2-x} \mathrm{NiO}_{4+\delta}$ after being sintered at $1100^{\circ} \mathrm{C}$ (space group I4/mmm): (a) $\mathrm{x}=0$; (b) $\mathrm{x}=0.05$; (c) $\mathrm{x}=0.10$; (d) $\mathrm{x}=0.15$. (+) represent the experimental data points, solid red line is refined data fit .76

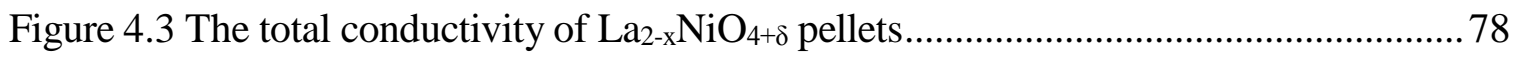

Figure 4.4 Temperature-dependent polarization cathode resistance of $\mathrm{La}_{2-\mathrm{x}} \mathrm{NiO}_{4+\delta}(\mathrm{x}=0-0.2) 80$ 
Figure 4.5 Temperature-dependent polarization cathode resistance of $\mathrm{La}_{2-\mathrm{x}} \mathrm{NiO}_{4+\delta}(\mathrm{x}=0-0.2) 80$

Figure 4.6 Temperature-dependent polarization cathode resistance of $\mathrm{La}_{2-\mathrm{x}} \mathrm{NiO}_{4+\delta}(\mathrm{x}=0-0.2)$

for high frequency arcs

Figure 4.7 Temperature-dependent polarization cathode resistance of $\mathrm{La}_{2-\mathrm{x}} \mathrm{NiO}_{4+\delta}(\mathrm{x}=0-0.2)$

for high frequency arcs. 81

Figure 4.8 Temperature-dependent polarization cathode resistance of $\mathrm{La}_{2-\mathrm{x}} \mathrm{NiO}_{4+\delta}(\mathrm{x}=0-0.2)$

for low frequency arcs

Figure 4.9 Temperature-dependent polarization cathode resistance of $\mathrm{La}_{2-\mathrm{x}} \mathrm{NiO}_{4+\delta}(\mathrm{x}=0-0.2)$ infiltrated LSCF.

Figure 4.10 Nyquist plot of three electrode cell with A-site deficient $\mathrm{La}_{2-\mathrm{x}} \mathrm{NiO}_{4+\delta}$ coated

$\mathrm{LSCF}$ cathode at $700^{\circ} \mathrm{C}$ after $-0.2 \mathrm{~V}$ cathodic polarization (a) $\mathrm{La}_{2} \mathrm{NiO}_{4+\delta}$, (b)

$\mathrm{La}_{1.95} \mathrm{NiO}_{4+\delta}$ and (c) $\mathrm{La}_{1.85} \mathrm{NiO}_{4+\delta}$ .85

Figure 5.1 Schemic of the aggregation and sintering process of particles...........................89

Figure 5.2 XRD pattern of $\mathrm{CeO}_{2} \& \mathrm{LNO}$ co-infiltrated LSCF/GDC/LSCF cells .................92

Figure 5.3 XRD pattern of $\mathrm{CeO}_{2} \& \mathrm{LNO}$ powders co-synthesized by sol-gel solution........92

Figure 5.4 SEM image of (a) and (b) as-prepared LNO-infiltrated LSCF electrode, (c) and

(d) LNO-infiltrated LSCF electrode with $1080 \mathrm{~h}$ heat treatment at $750^{\circ} \mathrm{C}$, (e) and (f) as-prepared 20 mol. \% $\mathrm{CeO}_{2} \& \mathrm{LNO}$ co-infiltrated electrode, (g) and (h) 20 mol. \% $\mathrm{CeO}_{2} \& \mathrm{LNO}$ co-infiltrated LSCF electrode with $1080 \mathrm{~h}$ heat treatment at $750^{\circ} \mathrm{C} .95$

Figure 5.5 Nyquist diagram of electrochemical impedance spectra for LNO infiltrated

LSCF symmetrical cells with and without $\mathrm{CeO}_{2}$ co-infiltration. 97

Figure 5.6 Arrhenius plots of fitted electrochemical impedance spectra for LNO infiltrated

LSCF symmetrical cells with and without $\mathrm{CeO}_{2}$ co-infiltration. 98

Figure 5.7 Nyquist diagram of electrochemical impedance spectra for LNO infiltrated LSCF symmetrical cells with and without $\mathrm{CeO}_{2}$ co-infiltration .98 
List of Tables

Table 3.1 The surface oxygen exchange coefficient $(k)$ and of $\mathrm{La}_{2} \mathrm{NiO}_{4+\delta}$, LSCF and LNOcoated LSCF (LNO/LSCF) cathode at $700^{\circ} \mathrm{C}$ under different oxygen pressure ........55

Table 3.2 Refined cell parameters and error value in I4/mmm space group .........................63

Table 3.3 Refined atomic position for pure LNO in I4/mmm space group............................63

Table 3.4 Refined atomic position for pure LNO in I4/mmm space group............................63

Table 3.5 Refined band length in $\mathrm{I} / \mathrm{mmm}$ space group ........................................................64

Table 3.6 The valence of transition metal and the amount of nonstoichiometric oxygen in

LNO-based materials by iodometric titration ...............................................................64

Table 4.1 Refined cell parameters and error value of A-site deficient LNO in I4/mmm

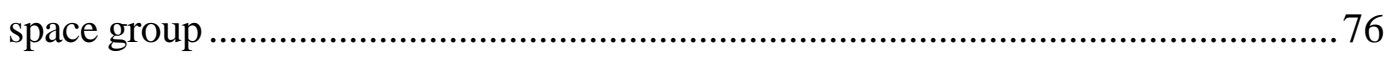

Table 4.2 Refined band length of A-site deficient LNO in I4/mmm space group .................76

Table 4.3 The valence of transition metal and the amount of nonstoichiometric oxygen in A-site deficient LNO materials by iodometric titration.............................................. 78

Table 4. 4 The ratio of $\mathrm{La} / \mathrm{Ni}$ in $\mathrm{La}_{2-\mathrm{x}} \mathrm{NiO}_{4+\delta}$ with and without long-term operation .............85 


\section{Chapter 1: Introduction and Research Objectives}

\subsection{Introduction}

Perovskite families of Sr-doped lanthanum cobaltite ( $\mathrm{La}, \mathrm{Sr}) \mathrm{CoO}_{3-\delta}$ (LSC) and ferrite $(\mathrm{La}, \mathrm{Sr})(\mathrm{Co}, \mathrm{Fe}) \mathrm{O}_{3-\delta}(\mathrm{LSCF})$ have advantages on high electrochemical activity for oxygen reduction at the intermediate temperature (IT) range $\left[{ }^{1},{ }^{2}\right]$. As LSCF and LSC readily become oxygen non-stoichiometric (deficient) at relatively low temperatures $\left(600^{\circ} \mathrm{C}\right)$ and ambient $\mathrm{PO}_{2}$, they show significant mixed electronic and ionic conductivity (MIEC) (within the ranges of $10^{2} \mathrm{~S} / \mathrm{cm}$ and $10^{-2} \mathrm{~S} / \mathrm{cm}$, respectively). This renders entire cathode surfaces electro-catalytically active for the oxygen reduction reaction (ORR) process, thus substantially improving electrode polarization activation. The technical advantages distinguish LSCF and LSC cathodes as prospective candidates for intermediate temperature solid oxide fuel cells (IT-SOFCs) operation over ( $\mathrm{La}, \mathrm{Sr}) \mathrm{MnO}_{3-\delta}$ (LSM), and attract intensive efforts of material optimization without necessitating the overhaul of existing industrially-viable electrode architecture.

Accompanying the performance promises, recent studies have identified that the ORR kinetics on LSCF and LSC type cathodes can be significantly limited by surface reaction processes and, consequently, the surface chemistry/structure of the materials are key to the electrode activity, which is sensitive to the electrode processing/operation histories. In particular, the activation/performance degradation of LSCF cathodes are found to strongly correlate to the enrichment/depletion of certain surface cations, which can be tailored by inherent segregation from cathode bulk and/or external material sources as functions of applied electrode over-potential and temperature. Cation-related surface chemistry studies have become the research focus of perovskite cathodes lately, and various advanced characterization techniques have been utilized for in-situ and ex-situ monitoring of the surface layer properties such as phase composition, conductivity, microstructure, elastic strain and oxygen exchange rates. Even with these progressive efforts in recent years, the mechanisms and effective resolution controlling the surface phase/structure transition of MIEC cathode oxides, especially under electrochemical 
operation conditions, remain an open question without unanimous agreement on experimental findings and even theoretical interpretation of fundamental observations with well-defined model electrodes and epitaxial thin-film structures. The lack of an effective method to improve the stability of LSCF calls for further investigation in which the surface properties are modified in a performance/stability directly relevant to practical SOFC operation.

The recently reported rapid oxygen reduction activity at the interface of $(\mathrm{La}, \mathrm{Sr}) \mathrm{CoO}_{3-\delta}$ (LSC113) and ( $\mathrm{La}, \mathrm{Sr})_{2} \mathrm{CoO}_{4}(\mathrm{LSC} 214)$ phases opened up new research questions for the potential role of hetero-structured interfaces in the development of advanced cathodes for solid oxide fuel cells (SOFCs). The investigations proved that many hetero-structured interfaces exhibit fantastic physical, chemical, and electrochemical properties including enhanced oxygen electro-catalytic activity and electronic/ionic conductivity compared with the nature of the bulk. Nickelates $\mathrm{Ln}_{2} \mathrm{NiO}_{4+\delta}(\mathrm{LNO}, \mathrm{Ln}=\mathrm{La}, \mathrm{Pr}, \mathrm{Nd})$ and related oxides with a Ruddlesden-Popper (RP) phase have recently shown promise as alternative ITSOFC cathode materials. Unlike oxygen-deficient perovskite counterparts in which oxygen exchange and mobility are dictated by oxygen vacancies, oxygen transport in nickelates predominantly occurs through interstitial oxygen over the entire temperature range for IT-SOFC operation. The high oxygen hyper-stoichiometry $(\delta>0.1)$ exhibited by nickelate-type materials provides rapid bulk oxygen diffusion and surface oxygen exchange properties.

The addition of a Ruddlesden-Popper (RP) ( $\left.\mathrm{La}_{0.5} \mathrm{Sr}_{0.5}\right)_{2} \mathrm{CoO}_{4+\delta}(\mathrm{LSC113})$ phase on the surface of the perovskite $\mathrm{La}_{0 .} \mathrm{Sr}_{0.2} \mathrm{CoO}_{3-\delta}$ (LSC214) significantly enhances the surface activity of the oxygen reduction reaction at the hetero-structured boundary between the two phases by 2-3 orders of magnitude. It has also been found that the LSC214 decoration can increase the oxygen surface exchange coefficient of the $\mathrm{La}_{0.6} \mathrm{Sr}_{0.4} \mathrm{Co}_{0.2} \mathrm{Fe}_{0.8} \mathrm{O}_{3-\delta}$ (LSCF113) by 2 times $\left[{ }^{3,4}\right]$, but it needs to be notice in these reports that LSCF113 (001) thin film has much larger oxygen surface exchange coefficient than LSC113. Meanwhile, density functional theory (DFT) calculations have shown that a large driving force exists in the inter-diffusion of A-site cationic across the hetero-structure interface, especially the lower diffusion energy of Sr from LSCF113 into LSC214Such inter-diffusion has been postulated 
to stabilize $\mathrm{Sr}$ in LSC214 and La in LSC113. The introduction of hetero-structured interfaces provides a new possible solution for $\mathrm{Sr}$ segregation in the development of advanced LSC/LSCF cathodes for solid oxide fuel cells (SOFCs).

Meanwhile, more active interfaces have been introduced in this hetero-structured cathode, and more complexity of reaction mechanisms is involved in the oxygen reduction reaction procedures. A number of investigations have been conducted to uncover the underlying mechanism of the high catalytic activity of these modified hetero-structured catalysts and tried to reveal reliable information to optimize electrodes and improve electrode performance and stability. However, so far the mechanisms of oxygen reduction and transport in different cathode interfaces are still indistinct in different types of single MIEC cathode materials, let alone in the hetero-structured cathode. Especially under polarization conditions, the variation of intrinsic properties of cathode materials is hard to detect due to the lack of advanced techniques operating under electrochemical conditions. The lack of intrinsic properties of materials and over-potential distribution theory under electrochemical conditions limits the understanding of the ORR mechanism and the optimization of electrochemical performance and stability of cathode. Therefore, a clear understanding of the function of electrochemical conditions, both on the intrinsic properties of materials and the corresponding ORR mechanisms, is one of the urgent issues in the development of advanced cathodes.

The main focus of this work is to develop an advanced hetero-structured cathode with remarkable stability and catalytic activity and to gain the fundamental understanding of over-potential on oxygen reduction reactions on the hetero-structured cathode. As is shown by a literature review on prior works, LNO oxides, as interstitial oxygen conductors, have a higher surface oxygen exchange coefficient together with an Sr-free chemical structure as compared to LSCF. Utilization of LNO as a surface catalyst on LSCF is believed to enhance the surface-limited ORR kinetics for LSCF as the result of a modified overall surface oxygen exchange coefficient and electrochemical reaction steps resulting from interstitial oxygen transport in LNO. Furthermore, the hetero-structured cathode surface can also be expected to maintain high activity under electrode polarization with fewer negative effects from surface cation segregation of $\mathrm{Sr}$, which is 
known to cause degradation issues for conventional LSCF and LSC cathodes, thus improving the cathodes' long-term stability. Meanwhile, this work opens a window into the deep understanding of the oxygen reduction reaction mechanism under electrochemical conditions. The relationship between thermodynamic and electrochemical theory has been discussed based on Nernst equations. Projecting a steady state to an equivalent equilibrium status is quite a common practice, e.g. calculating the effective oxygen activity in a particular component under operation conditions from the over-potential based on the Nernst equation. It finds a wide application in SOFCs electrochemistry, such as yielding critical initial parameters for the modeling studies or providing evaluation of the stability of electrodes under loading. In fact, the equivalency casts influence the kinetics of electrochemical reactions as well, mediating the exchange rate at equilibrium status by the balanced reactant compositions vs. mediating the net reaction rate in a steady state by the electrical potential. Then, it appears interesting to ask whether such equivalency based on the thermodynamic principle, i.e. the Nernst equation, can be applied to the kinetics to show that the reaction rate for a steady state (net rate) is related to that under an equivalent equilibrium status (exchange rate).

\subsection{Research Objectives}

The primary objectives of this project are to (1) develop a cathode with a hetero-structured surface that possesses high performance and stability via a low-cost infiltration method; (2) develop a new method to inhibit the deadly issues of nanoparticles aggregation/growth and delamination in infiltration techniques, and (3) develop the fundamental understanding of ORR mechanisms under open circuit voltage (OCV) and electrode polarization conditions, especially the equivalence relation between thermodynamic and electrochemical driving force on the cathode reaction through systematic experimental investigations and theoretical modeling. The objectives can be further illustrated as below.

In this work, a high performance and stable SOFCs cathode with a hetero-structured surface is developed by infiltration techniques. The commercial cathode materials for ITSOFCs, $\left(\mathrm{La}_{0.6} \mathrm{Sr}_{0.4}\right)_{0.95} \mathrm{Co}_{0.2} \mathrm{Fe}_{0.8} \mathrm{O}_{3-\delta}$ (LSCF), can serve as the backbone. A novel infiltrated material nickelate $\mathrm{A}_{2} \mathrm{BO}_{4+\delta}$, which possesses high ionic conductivity due to interstitial 
oxygen, is used as the surface layer to improve the electrode performance and surface stability. The correlation parameters of infiltration processes, such as infiltration solution composition, sintering temperature and loading amount et al. have been optimized to achieve the best cathode stability and electrochemical performance. The initial electrochemical performance has been evaluated by electrochemical testing of symmetrical and full cells. Long-term on-cell testing is planned to check the stability of the optimized cathode. Furthermore, fundamental mechanisms of oxygen reduction reaction behavior in the hetero-structured cathode have been studied by experimental investigation, with the focus on the hetero-structured surface and its effect on oxygen exchange behavior between the surface and the bulk of LSCF cathodes. Degradation of oxygen reduction ability for the infiltrated cathode has been quantitatively characterized by electrochemical measurement, electrical conductivity relaxation, energy dispersive X-ray detector and X-ray photoelectron spectroscopy. The electrical conductivity relaxation technique serves as the main experimental method to characterize oxygen transport behavior in the cathode especially at the infiltrated/cathode backbone interface. In addition, the fundamental understanding of oxygen reduction reaction mechanism on the different interfaces under electrochemical conditions is detected to reveal the underlying mechanisms of high catalytic activity in the hetero-structured cathode and provide valuable information in the cathode optimization.

\subsection{Experimental Methodology}

\subsubsection{Infiltrated Hetero-Structured Cathode Materials Preparation}

The infiltration precursor solution with a concentration of $1 \mathrm{~mol} / \mathrm{L}$ was composed of stoichiometric nitrates (Alfa Aesar) dissolved in a mixture of ethanol and deionized water with a volume ratio of 1:0.6. The appropriate amount of ethanol can promote the formation of the infiltration phase and improve its distribution status on cathode substrate. After infiltrating the nitrate solution into each side of a porous cathode backbone, the cells were sintered at different temperatures for $1 \mathrm{~h}$ to allow the formation of $\mathrm{s}$ single infiltration phase on the cathode backbone. Repeated infiltration followed by firing process was carried out to increase the amount of infiltration phase in the modified cathode. 


\subsubsection{Dense Hetero-Structured Cathode Layers Fabrication}

The investigated powders were synthesized by the sol-gel method. Stoichiometric nitrates (Alfa Aesar) were dissolved into distilled water separately together with complexing agent citric acid (Alfa Aesar). The mixed solution was held at $80^{\circ} \mathrm{C}$ and stirred until gelation. The gel was heated up to $600^{\circ} \mathrm{C}$ to decompose nitrates and organic residuals. Then, the resultant powders were sintered in air at $1100^{\circ} \mathrm{C}$ for $4 \mathrm{~h}$ and examined by $\mathrm{X}$-ray diffraction to check phase purity.

The resultant powders along with polyvinyl butyral (PVB) and fish oil at the weight ratio of 100:2:1.5 was added into ethanol (5 g powders to $30 \mathrm{ml}$ ethanol), ball-milled for $24 \mathrm{~h}$ to produce suspension, and then air-brushed onto both sides of the electrolyte to fabricate a thin layer. In brief, the electrolyte in $1.5 \mathrm{~g}$ was dry pressed under $50 \mathrm{MPa}$ in the die with a diameter of $16.5 \mathrm{~mm}$. The suspension was evenly sprayed onto the electrolyte surface, followed by an intermediate pressing of $200 \mathrm{MPa}$. The spray process was repeated, followed by a final pressing at $300 \mathrm{MPa}$. Electrode/electrolyte/electrode sandwiches were sintered to $1300^{\circ} \mathrm{C} 4 \mathrm{~h}$ for densification. In order to avoid any possible element enrichment in the surface, the sintered pellets were polished manually using sandpaper and polishing cloth of $2 \mu \mathrm{m}$. The removed thickness for each sample could not be precisely controlled, but was estimated at about $50 \mu \mathrm{m}$ after inspection by scanning electron microscopy (SEM, Hitachi S-4700F), depending on individual samples.

\subsubsection{Symmetrical Cells and Fuel Cells Assembling}

A symmetric cell configuration of LSCF|GDC|YSZ|GDC|LSCF was assembled starting with the preparation of $8 \% \mathrm{Y}_{2} \mathrm{O}_{3}$ - stabilized $\mathrm{ZrO}_{2}$ (YSZ, TZ8Y, Tosoh, Japan) electrolyte supports. A tape casting technique followed by sintering at $1450^{\circ} \mathrm{C}$ for $5 \mathrm{~h}$ was used to form dense YSZ electrolyte pellets with a thickness of about $300 \mu \mathrm{m}$. Then, identical GDC buffer layers were screen printed symmetrically on both sides of the YSZ electrolyte and then sintered at $1300^{\circ} \mathrm{C}$, to avoid interactions between the YSZ electrolyte and the LSCF cathode. A-site deficiency $\left(\mathrm{La}_{0.6} \mathrm{Sr}_{0.4}\right)_{0.95} \mathrm{Co}_{0.2} \mathrm{Fe}_{0.8} \mathrm{O}_{3-\delta}$ (LSCF) powder from Fuel Cell Material Co. was dispersed into an organic vehicle ink (VHE, Fuel Cell Material Co. in US.) to form a slurry. Porous LSCF layers with a thickness of 10-20 $\mu \mathrm{m}$ were deposited on 
the GDC buffer layers by screen printing. Eventually, the bonded layers were co-fired at $1150^{\circ} \mathrm{C}$ for $3 \mathrm{~h}$ to obtain the symmetric cell using the LSCF cathode with an active surface area of about $0.7 \mathrm{~cm}^{2}$. For comparison and performance evaluation, LNO was infiltrated into both sides of the LSCF layers in the LSCF|GDC|YSZ|GDC|LSCF cell, as described below, to form a symmetric cell using LNO infiltrated LSCF cathodes. Pt meshes were attached to both electrode sides of the symmetric cells by using Pt paste as current collectors. For comparison, anode-supported fuel cells using LSCF or LNO infiltrated LSCF cathodes were prepared and measured to form NiO-YSZ|YSZ|GDC|LSCF and NiOYSZ|YSZ|GDC|LNO-infiltrated LSCF. Anode-supported half fuel cells Ni-YSZ/YSZ were commercially available (MSRI, Salt Lake City, UT) and consisted of a $\sim 10 \mu \mathrm{m}$-thick YSZ electrolyte and $750 \mu \mathrm{m}$-thick Ni-YSZ anode. The GDC buffer layer and bare/modified LSCF cathodes were sequentially applied to the YSZ electrolyte using the same procedures for the fabrication of symmetric cells as mentioned above. The whole cell was then sealed using mica material gaskets, attached using Pt meshes with Pt paste for cathode and $\mathrm{Ni}$ paste for anode, and fixed in a conventional single cell test stand (installed at NETL, Morgantown, WV site).

\subsubsection{Oxygen Reduction Reaction Activity Measurements}

Electrochemical impedance spectroscopy (EIS) is common in situ high temperature and high pressure electrochemical techniques, which can provide great insight into how an electrode performs. This technique has been widely utilized for cathodic oxygen reduction reaction (ORR) studies. EIS possesses the ability to evaluate how sensitive each electrode is to temperature, pressure and bias voltages. Also it can be utilized to elicit various reaction mechanisms.

The electrochemical performance of the hetero-structured cathode was characterized by EIS through Solartron $1286 \& 1260$ or Autolab Model 2000. EIS measurements were collected on a large variety of our cathode samples under different oxygen partial pressure $\left(1 \mathrm{~atm}\right.$ to $\left.2 * 10^{-4} \mathrm{~atm}\right)$ and bias voltages $(-0.2 \mathrm{~V}$ to $0.2 \mathrm{~V})$. The designed oxygen partial pressure was produced by mixing oxygen or air with $\mathrm{N}_{2}$, controlled via mass flow meters (Alicat Scientific). A $10 \mathrm{mV}$ AC perturbation signal was applied over the frequency range 
from $0.01 \mathrm{~Hz}$ to $1 \mathrm{MHz}$ in each measurement. The obtained EIS spectra were deconvoluted and analyzed using Z-view software. Specific EIS experimental details were provided within each respective chapter.

The conductivity relaxation technique involves measurements of time variations of the electrical conductivity of a sample after a stepwise change in the ambient oxygen partial pressure. To determine oxygen surface exchange (k) and bulk diffusion coefficients (D), a correlation between a mean conductivity and the corresponding mean non-stoichiometry had been established.

$$
\frac{\delta(t)-\delta(0)}{\delta(\infty)-\delta(0)}=\frac{\sigma(t)-\sigma(0)}{\sigma(\infty)-\sigma(0)}
$$

$\delta(0), \delta(\infty)$, and $\delta(t)$ are separately standing for initial, final and time-related nonstoichiometry values. $\sigma(0), \sigma(\infty)$, and $\sigma(t)$ are conductivities correspond to $\delta(0), \delta(\infty)$, and $\delta(t)$, respectively.

Based on Fick's second law $\left(\frac{\partial C}{\partial t}=D \frac{\partial^{2} C}{\partial x^{2}}\right)$, the relationship between conductivity and time can be established:

$$
\begin{gathered}
\frac{\sigma(t)-\sigma(0)}{\sigma(\infty)-{ }^{(0)}}=1-\sum_{n=1}^{\infty} \frac{2 L^{2} \exp _{2}\left(-b^{2} D t / a^{2}\right)}{b_{n} b_{n} L L} L \\
L=\frac{a}{l}=\frac{a k}{D}=b_{n} \tan b_{n}
\end{gathered}
$$

Here, $a(\mathrm{~cm})$ is the half thickness of the tested pellet sample in the diffusion solution. $L$ is a unit less parameter from which the characteristic thickness $l_{\mathrm{c}}(\mathrm{cm})$ can be derived as shown in the above equation. $b_{\mathrm{n}}$ is a parameter generated during the process solving Fick's second law.

Keithley 2400 was used to offer the applied constant current and the corresponding voltage was measured by Keithley $2182 \mathrm{~A}$. The oxygen partial pressure ( $0.21 \mathrm{~atm}$ to $0.02 \mathrm{~atm}$ ) was 
obtained by mixing air with nitrogen. The gas flow rate and other data were collected by using LabVIEW software.

\subsubsection{Ex situ Characterization}

X-ray diffraction patterns were gathered by a Panalytical X'Pert PRO diffractometer with $\mathrm{Cu} \mathrm{K \alpha}$ radiant. The phase composition of samples was analyzed by Jade 5 and GSAS refinement software.

The surface morphology and cross section microstructure of the electrodes and cells were observed by using scanning electron microscope (JEOL JSM-7600F), and elements distribution and diffusion of the hetero-structured electrode were characterized with an Oxford Inca energy-dispersive X-ray spectrometry (EDS) system equipped with SEM. X-ray photoelectron spectroscopy (XPS) is a surface sensitive technique that analyzes the top 25 to 50 angstroms of a sample exterior. The XPS measurements were carried out using a Physical Electronics, PHI 5000 Versa Probe (XPS/UPS) spectrometer (Chanhassen, MN) with a monochromatic $\mathrm{Al} \mathrm{K} \alpha$ source operated at $300 \mathrm{~W}$ and a base pressure of $1 * 10^{-7}$ Torr. Transmission electron microscopy (TEM) observations and elemental identifications were carried out on a JEOL JEM-2100 LaB6 microscope equipped with an energy dispersion spectrometry (EDAX, Evex Nano Analysis). 


\section{Chapter 2: Literature Review}

There is a consensus that global warming is occurring as a result of effluent gas emission, mainly $\mathrm{CO}_{2}$. Global surface temperatures have been increasing at an average of almost $0.6^{\circ} \mathrm{C} /$ century for the past century, and this trend has rapidly increased in the past 30 years based on reports from some well-known environments monitoring centers $\left.{ }^{5}\right]$. Nevertheless, the world population still keeps growing at a rate of 1.2-2\% per year. For economic development to keep up with the population explosion, global energy demands are expected to increase up to an order of magnitude by 2050, meaning, primary-energy demands are expected to increase by 1.5 to 3 times $\left[{ }^{6}\right]$. With worldwide oil supplies shrink, governments and researchers have put relentless effort toward the development of new substitutable power generation technologies to meet the fuel needs of a rising population. The exploitation of clean and regenerative energy is one of the major challenges for researchers. Fuel cells converting chemical energy of different fuel gas reaction directly into electrical energy are efficient and environmentally clean, since no combustion reaction is involved in conversion $\left[{ }^{7}\right]$.

\subsection{Solid Oxide Fuel Cells}

\subsubsection{Introduction and Mechanism}

The utilization of a solid electrolyte and higher operating temperatures (typically at 700$900^{\circ} \mathrm{C}$ ) for solid oxide fuel cells (SOFCs) are a benefit with respect to efficiency, reliability, modularity, fuel flexibility, and environmental friendliness as compared with other conventional power-generating systems $\left[^{8}\right]$. In addition, an SOFC system usually operates at extremely high temperatures $\left(600-1000^{\circ} \mathrm{C}\right)$. Such high operating temperatures allow internal reforming, facilitates rapid electro-catalysis with non-precious metals and produces high quality byproduct heat for co-generation, thereby enhancing the efficiency up to approximately $70 \%$ with an additional $20 \%$ as heat recovery [ $\left.{ }^{9}\right]$.Noble metals-free of SOFCs provide a competitive price and a potential long life expectancy of more than 40000-80000 h $\left.{ }^{10}\right]$. Hence, SOFC technology is extremely suited to applications in either 
full-scale industrial stations or large-scale electricity-generating stations. Some Toyota Company developers are also expected to use SOFCs in motor vehicles $\left[{ }^{11-13}\right]$.

An SOFC consists of two electrodes separated by a hard ceramic electrolyte such as the remarkable ceramic material called zirconia. Hydrogen or other hydrocarbon fuels are fed into the fuel cell through anode, and oxygen, usually from air, flows into the cathode of the cell. When a load is placed on the external circuit between cathodes and anodes, electrons flow from anodes to cathodes. Additionally, a continuous supply of oxygen ions flows through the internal circuit due to (1) oxygen concentration gradient between cathode and anode and (2) overall electrical charge balance. Thereby, a useful electrical power is generated by the combustion of the fuel, as shown in Fig $2.1\left[{ }^{14}\right]$. The only byproduct of the whole processes with hydrogen fuel is water and heat from the exothermic reaction. Reforming natural gas or other hydrocarbon fuels to extract the necessary hydrogen can be accomplished within the fuel cells, eliminating the need for an external reformer, as is the case for other types of fuel cells.

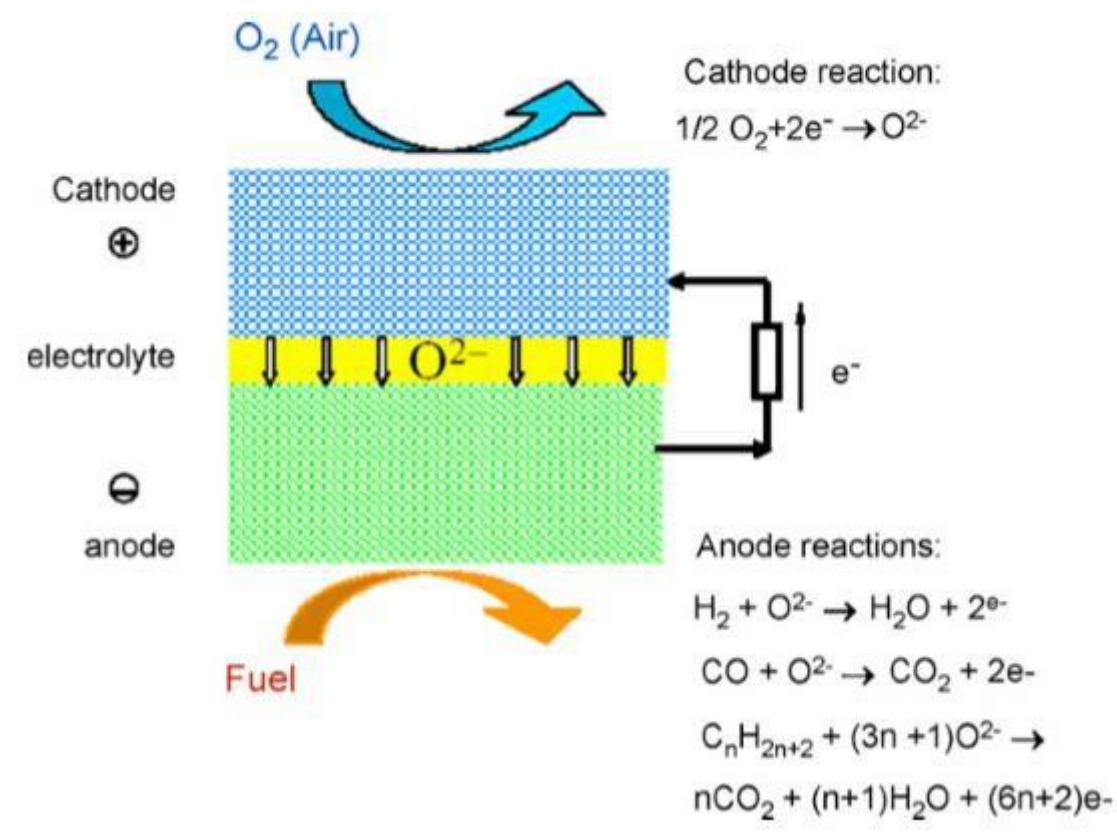

Figure 2.1 Schematic of an electrolyte supported single SOFC cells

The SOFCs reactions include:

Anode side: 


$$
\begin{gathered}
\mathrm{H}_{2}+\mathrm{O}^{2-} \rightarrow \mathrm{H}_{2} \mathrm{O}+2 e \\
\mathrm{CO}+\mathrm{O}^{2-} \rightarrow \mathrm{CO}_{2}+2 e \\
\mathrm{CH}_{4}+4 \mathrm{O}^{2-} \rightarrow 2 \mathrm{H}_{2} \mathrm{O}+\mathrm{CO}_{2}+8 e
\end{gathered}
$$

Cathode side:

$$
O_{2}+4 e \rightarrow 20^{2-}
$$

All the above electrochemical processes are driven by the gradient of the oxygen ions concentration/ oxygen partial pressure $\left(\mathrm{PO}_{2}\right)$ /oxygen chemical potential across the electrolyte. Thus, SOFCs can also be called oxygen concentration cells. The theoretical reversible voltage under open circuit, EOCv, is determined by the standard electrode potential and the different oxygen concentration between two electrodes, as given by Nernst equation:

$$
E_{\text {OCV }}=E^{\Theta}+\frac{R T}{4 F} \ln \left(\frac{P_{O} \text { cathode }}{P_{O_{2}} \text { anode }}\right)
$$

Where $\mathrm{R}$ is the universal gas constant, $\mathrm{T}$ is temperature in degrees Kelvin, and $\mathrm{F}$ is Faraday's constant $(96500 \mathrm{C} / \mathrm{cm})$. The coefficient 4 represents the number of transferred electrons per mole of oxygen molecule reduced in the cell. The $\mathrm{PO}_{2}$ at the cathode is 0.21 atm when air used as an oxidant through the cathode side of SOFCs. In laboratory experiments, the commonly used hydrogen gas with steam is generally fed into the anode. Consequently, the $\mathrm{PO}_{2}$ at the anode can be deduced based on the equilibrium constant and the Gibbs free energy of the following equation:

$(\mathrm{g}) \Leftrightarrow H$

$$
H(g)+{ }_{-}^{1} O O(g) \quad(2.6
$$

$2^{2} 2^{2} \quad 2$

Therefore, at the anode side, the change in Gibbs free energy and oxygen partial pressure can be expressed using Equations (2.6-2.8) by assuming all the water exists in the form of steam during the gas delivery process between the water bubbler and the SOFC anode,

$$
K_{e q}=\frac{P_{H}^{P_{H}}{ }_{\mathrm{H}_{2}}\left(\mathrm{P}_{\mathrm{O}_{2}}\right)^{1 / 2}}{P_{2}}
$$




$$
\Delta G_{T}=\Delta H_{T}-T \Delta S_{T}=-R T \ln \left(K_{e q}\right)
$$

Oxygen partial pressure can be predicted as:

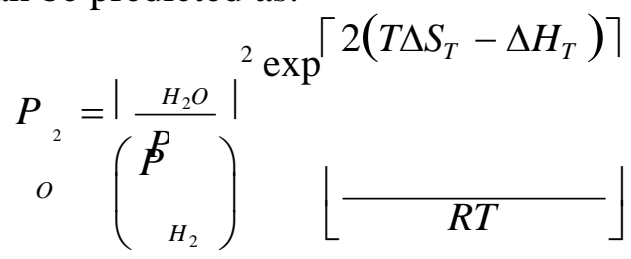

Here, $\mathrm{K}_{\mathrm{eq}}$ is the equilibrium constant of Equation (2.6), $\Delta H_{T}$ is heat change, and $\Delta S_{T}$ is entropy change. The theoretical Nernst potential at any SOFC operation temperature can be estimated by substituting $\mathrm{PO}_{2}$ in Equation (2.9) and $\mathrm{PO}_{2}$ of cathode in Equation (2.5) $\left[{ }^{15}\right]$. However, the actual cell voltage obtained under operation conditions is always lower than the theoretical Nernst value $\left[{ }^{16}\right]$. The departure of the operating voltages from the theoretical values due to the passage of faradaic current is defined as polarization. Overpotential $\eta$ can be indicative of the extent of polarization, which is induced by ohmic loss $\left(\eta_{\text {ohm }}\right)$, charge transfer (activation) polarization $\left(\eta_{\text {act }}\right)$ or diffusion (concentration) polarization $\left(\eta_{\mathrm{con}}\right)$.

$$
\eta=\eta_{\mathrm{oh} m}+\eta_{c o n}+\eta_{a c t}
$$

Ohmic loss arises from the resistance to ionic transport flow in ionic conductors or electrons transport in electronic conductors or interfacial contact between cell components, i.e., electrodes and electrolyte. The main contribution of the ohmic polarization is the slow ionic transport in electrolyte material, which is directly proportional to the magnitude of current flowing through the cell based on ohm's law $\Delta E=i R_{s}\left[{ }^{17,18}\right]$. Activation polarization occurs when reacting chemical species are involved in electrode reaction[ $\left.{ }^{10}\right]$. Thus, for an electrode chemical reaction, the activation energy barrier of the rate limiting step of the reaction happening in multiple steps has to be overcome, which requires an extra potential energy. And the chemical reaction rate is governed via the Butler-Volmer relation:

$$
i=i_{0}|\exp | \frac{\alpha n F \eta_{a c t}}{R T}\left|-\exp \left(\frac{\left.\left.-(1-\alpha) n F \eta_{a c t}\right)\right\rceil}{R T}\right)\right|
$$

Where $i_{0}$ is the exchange current density, $\alpha$ is the transfer coefficient, $\mathrm{n}$ is the number of electrons taking part in the reaction at the electrode, and $\eta_{\text {act }}$ is the activation over- 
potential. This equation relates to the rate of charge transfer process reacting at the 
electro-catalyst (electrode)/electrolyte and electro-catalyst (electrode)/gas interface and representing with current density to the activation over-potential. Activation polarization is typically an issue occurring in cathodes. This is attributed to the slow oxygen reduction reaction kinetics which involves the breaking of high strength bonds present in oxygen molecules. Thus, the reaction rate using oxygen gas as an oxidizer (at the cathode) becomes slower by several orders of magnitude compared with fuel oxidation reactions at the anode $\left[{ }^{15,17}\right]$. Concentration polarization occurs when the reacting molecules are consumed at a faster rate, or when products are not removed quickly enough from the reaction sites. This lowers the concentration of reactant species, i.e., low oxygen partial pressures at the cathode or low partial pressure of fuel at the anode compared with the bulk supply. Such a behavior leads to a reduction in the voltage output, and creates a significant disadvantage when high current is drawn from the SOFC. In extreme cases, the concentrations of reacting molecules reduce to zero at the reaction site, current drops to a limiting unsustainable value, and the fuel cell ceases to run. These losses cannot be eliminated, but it is possible to minimize these losses by incorporating a proper choice of materials, organized microstructural engineering, and optimized fabrication/or design of each cell component $\left[{ }^{18}\right]$.

In order to delineate the significance of SOFC components, the sequence of processes supported at each component of SOFC must be isolated. Hence, it becomes essential to assess the reactions occurring at each of the electrodes, the oxide diffusion through electrolyte, passage of electronic current through interconnects, and the separation of fuel and oxidizer through sealants. Thus, in the following sections each cell component of SOFC will be described based on their functionality, material and microstructural aspects, and their associated problems.

\subsubsection{Component Requirements}

Traditionally, an yttrium-stabilized zirconia (YSZ) electrolyte, lanthanum strontium manganite (LSM) cathode and a Ni-YSZ anode comprise the major parts of SOFCs, which needs a high operating temperature $\left(1000^{\circ} \mathrm{C}\right)$ to ensure a good performance $\left.{ }^{19}\right]$. However, the main challenges of the operation of SOFCs at high operating temperatures $\left(800-1000^{\circ} \mathrm{C}\right)$ 
include: (1) extended startup times since a period of fuel burning is required in order to reach the operating temperatures; (2) exert sealing problems due to the coefficient of thermal expansion (CTE) mismatch of adjoining materials; (3) the requirement of expensive interconnect and integration of materials for SOFC stacks for the tapping of useful power; (4) thermal stresses at electrolyte-electrode interfaces; as well as (5) elements inter-diffusion between cell components $\left[{ }^{10}\right]$. High temperature thermal cycles not only induce a substantial thermal stress on the materials, but also increase the diffusion kinetics which can change the chemistry of the adjoining components. Hence, these aspects must also be kept under consideration during SOFC operation. Therefore, an effective approach to solve these problems is to reduce the operating temperature to $600-800^{\circ} \mathrm{C}$. An added bonus of lowered operating temperatures is the possibility of direct (and cleaner) oxidation of methane without any carbon deposition, which would eliminate the need of reforming fuel. For these reasons, recent research focus has been towards lowering the operating temperature of SOFC. Unfortunately, lowering the operating temperature induced poorer cell performance due to increased polarization resistances of the electrode reactions and decreased electrolyte conductivity, and the barriers to low operating temperatures are many. To gain a better insight into lowering the operating temperature of SOFC, we must first understand the construction of SOFC, the various reactions mechanism and energy losses that occur in the different components of SOFCs.

\subsubsection{Anode}

The porous anode provides electrochemical oxidation reaction sites of the fuel, offers a path for released electrons to be transferred from the gas/electrolyte/anode reaction sites to the current collector in SOFC stacks, and allows the fuel and by-products to be delivered and removed from the surface sites. It must show high electro-catalytic characteristics toward oxidation of fuel gases, and rapid electrochemical catalysis on the hydrocarbon reforming. The desired anode is expected to be a mixed electronic and ionic conductor firstly with remarkable electronic conductivity to allow the passage of electrons. Secondly the excellent dimensional and chemical stability is expected for anode materials selection in reducing atmospheres at high operating temperature of SOFCs. And except for self- 
stability of anode materials under lower oxygen partial pressures, chemical compatibility and CTE matching with neighboring components are required for anode materials selection. Besides, anode materials are also supposed to display excellent resistance on carburization and sulfidation issues. $\left[{ }^{20}\right]$.

Porous composite $\mathrm{Ni} / \mathrm{YSZ}$ is currently the most common anode material for SOFC applications due to powerful electro-catalytic property and low cost. Metal nickel provides electronic conductivity and excellent electro-catalytic activity for hydrogen oxidation and natural gases reforming. The addition of YSZ adds ionic conductivity and helps create a suitable CTE, thus effectively broadening the triple phase boundary (TPB) areas and enhancing the dimensional stability. Moreover, $\mathrm{Ni}$ and YSZ are chemically stable in reducing atmospheres, essentially immiscible with each other and non-reactive in a wide temperature range. In the development of Ni-YSZ anode, excellent low anode overpotential has been achieved by optimizing the microstructure $\left.{ }^{21,22}\right]$. Two prominent drawbacks, poor carbon and sulfur resistances, limit the widely application of Ni-YSZ anode cermet. Carbon deposition on nickel particles surface give rise to the rapid deterioration of cell performance with dry methane and other hydrocarbon fuel gases due to the incomparable excellent electro-catalyst of nickel and insufficient steam and transported oxygen ions in anode. Another limitation to the use of hydrocarbon gas, as fuel, results from poor sulfur tolerance of Ni-YSZ anode. The deactivation from sulfur poisoning occurs due to strongly adsorption of $\mathrm{H}_{2} \mathrm{~S}$ on active sites of nickel, and thereby leads to the substantial rapid anode failure occurring at TPBs $\left[{ }^{23,24}\right]$.

In the search for alternative anode materials with fuel flexibility and capability of resisting sulfur contamination and carbon deposition, ceria-based anodes are widely confirmed to be effective in inhibiting carbon deposition since ceria has long exhibited excellent electrocatalyst on methane oxidation reaction. Both doped and un-doped ceria are provided with mixed ionic and electronic conducting at low oxygen partial pressures, which offers sufficient oxygen ions and directly converts the methane to carbon oxide and water. Therefore, they are benefit for the utilization of hydrocarbon fuels with a low steam content $\left[{ }^{25}\right]$. And to further improve the stability and performance of anode, the addition of metal $\mathrm{Cu}$ in ceria based anode have been investigated because $\mathrm{Cu}$ shows high electronic 
conductivity and excellent carbon deposition resistance. These composite anodes exhibits better electrochemical performance and higher resistance to carbon deposition than most current available conventional anode materials for SOFCs $\left[{ }^{26}\right]$. Meanwhile, some perovskite oxides with mixed ionic and electronic conductivity have attracted considerable attention. Lanthanum chromite has been firstly explored as anode materials due to its appropriate thermal and chemical stability. A site $\mathrm{Sr}$ and $\mathrm{B}$ site $\mathrm{Ti}, \mathrm{V}, \mathrm{Nb}$, Mo and $\mathrm{W}$ substitution in $\mathrm{LaCrO} 3$ display excellent resistance to carbon deposition even though its weak activity upon the methane reforming is not appreciably enhanced $\left[{ }^{14}\right]$. Recently, Goodenough and co-workers have reported that the double perovskite $\mathrm{Sr}_{2} \mathrm{Mg}_{1-\mathrm{x}} \mathrm{Mn}_{\mathrm{x}} \mathrm{MoO}_{6-}$ xcould provide remarkable long-term stability and tolerance to sulfur as anode materials with natural gas as the fuel in the operating temperatures range of $650-1000^{\circ} \mathrm{C}$ with $\left[{ }^{27}\right]$. Further modification of the double perovskite $\mathrm{Sr}_{2} \mathrm{Mg}_{1-\mathrm{x}} \mathrm{Mn}_{\mathrm{x}} \mathrm{MoO}_{6-\mathrm{x}}$ by $\mathrm{La}$, $\mathrm{Pr}, \mathrm{Ba}, \mathrm{Sr}$ doping on A-site and $\mathrm{Co}, \mathrm{Fe}, \mathrm{Mn}$ substitution on B-site have been gradually valued by researchers $\left[{ }^{28}\right]$.

Although progressive efforts have been made in the development of alternative anode materials, significant problems remain, particularly with the low resistance to carbon deposition and sulfur poisoning. Nevertheless, the desirable catalytic activity comparable with nickel and the high electronic conductivity properties warrant their further study.

\subsubsection{Electrolyte}

An electrolyte in SOFCs provides transmission medium for reduced oxygen ion and conducts it from cathode side to anode side where it reacts with hydrocarbons to produce $\mathrm{H}_{2} \mathrm{O}$ and $\mathrm{CO}_{2}$, and thereby completes the overall electrochemical redox reaction. A thermally activated process with oxygen vacancy hopping mechanism was assumed for oxygen ions conduction in the electrolyte. Large interionic open space, combined with low migration enthalpy less than $1 \mathrm{eV}$, is desired to achieve a high level of point defect hopping in the electrolyte crystal structure. Therefore, the main requirements for an efficiently electrolyte are: (1) sufficiently high oxygen ion conductivity $(0.1 \mathrm{~S} / \mathrm{cm}$ at operating temperature); (2) extremely low electronic transference properties to avoid current leaking $\left(<10^{-3}\right)$; (3) terrific thermodynamic and chemical stability in a wide range of temperatures 
(room temperature- $1000^{\circ} \mathrm{C}$ ) and oxygen partial pressures $\left(1-10^{-22} \mathrm{~atm}\right)$; (4) chemically inert toward both electrode materials under operating conditions; (5) compatibility of CTE with neighboring cell components; and (6) sufficient mechanical strength (>400 MPa) $\left[{ }^{29}\right]$.

A variety of ceramic materials have been employed as solid non-porous electrolyte in current technology. Stabilized zirconia with oxygen ions conductivity, especially yttriumstabilized zirconia (YSZ, $\left.\left(\mathrm{ZrO}_{2}\right)_{0.92}\left(\mathrm{Y}_{2} \mathrm{O}_{3}\right)_{0.08}\right)$, scandium-stabilized zirconia (ScSZ, $\left.\left(\mathrm{ZrO}_{2}\right)_{0.90-0.88}\left(\mathrm{Y}_{2} \mathrm{O}_{3}\right)_{0.10-0.12}\right)$, are the most common electrolyte to date. Adding Y or Sc into the fluorite structure-based zirconia introduces more oxygen vacancy defects and inhibits the phase transformation successfully. The ionic conductivity of ScSZ is much higher than that of YSZ at high temperature. The higher conductivity of $\mathrm{ScSZ}$ is attributed to the similarity of ionic size between $\mathrm{Zr}^{4+}$ and $\mathrm{Sc}^{3+}\left(\mathrm{Y}^{3+}>\mathrm{Sc}^{3+}>\mathrm{Zr}^{4+}\right)$ that leads to a smaller energy for defect association, and thereby increases oxygen ions mobility. However, the conductivity advantage disappears below $500^{\circ} \mathrm{C}$, becoming similar to or then lower than that of YSZ. Moreover, phase transformations in ScSZ appear from a distorted fluorite phase to a tetragonal phase after 5000h aging, and the conductivity of ScSZ decreases to the same level as that of YSZ, which was initially about twice as high as that of YSZ $\left.{ }^{30-32}\right]$. Similar fluorite structure-based ceria is another common electrolyte selection for SOFCs. Compared to pure zirconia, ceria owns much more intrinsic oxygen defects, particularly at low temperatures, and thereby can be a potential electrolyte material for IT-SOFCs. To increase the concentration of oxygen vacancy defects, ceria is also doped with low valence elements. The rapid oxygen transport properties have been obtained for gadolinium and samarium (GDC and SDC) doping with the lowest size mismatch with cerium. The primary disadvantage of doped-ceria is current leaking in the internal cells and reduction of the open circuit voltage that result from electronic conduction at low oxygen partial pressure $\left[{ }^{33,34}\right]$. Another class of fluorite-type structure electrolytes, based on $\mathrm{Bi}_{2} \mathrm{O}_{3}$, also has a highly deficient oxygen sub-lattice at low temperature. Unfortunately, $\mathrm{Bi}_{2} \mathrm{O}_{3}$-based materials possess a number of disadvantage to prevent the application in solid oxide fuel cells, including decomposition in reducing atmospheres, a high corrosion activity and low mechanical strength $\left[{ }^{35,36}\right]$. 
The perovskite, $\mathrm{LaGaO}_{3}$, doped with strontium and magnesium, $\mathrm{La}_{1-\mathrm{XSrxGa}} \mathrm{Sry}_{1-\mathrm{yg}} \mathrm{Og}_{3}$ (LSGM), is another serial electrolyte materials for low-temperature or intermediate SOFCs. The conductivities of LSGM is higher than that of YSZ and ScSZ at low temperature. Even though LSGM has similar to or lower conductivity than that of GDC, it is still superior to GDC as electrolyte in low oxygen partial pressures due to excellent chemical stability and inertia. Meanwhile, the chemical compatibility LSGM with common perovskite electrodes is much better than YSZ and GDC since similar crystal structure and elements introduce simple mutual inter-diffusion of elements instead of forming an insulting second phase in YSZ or GDC electrolyte. However, restriction on the processing conditions is a challenge for LSGM formation of a pure single-phase perovskite structure. And one of the challenges for LSGM electrolytes application is the development of cost-effective processes to fabricate the desired single-phase structure $\left[{ }^{37,38}\right]$.

Aluminum or magnesium doped $\mathrm{La}_{10} \mathrm{Si}_{6} \mathrm{O}_{27}$ with the apatite structure have also been investigated. The ionic conduction properties of doped $\mathrm{La}_{10} \mathrm{Si}_{6} \mathrm{O}_{27}$ were enhanced by increasing non-stoichiometric oxygen species, which suggests an interstitial ionic conduction mechanism $\left[{ }^{39,40}\right]$. And the pyrochlore structure electrolytes have also been investigated for potential electrolyte materials. Similar structure with the cubic fluorite ceria and zirconia could provide one oxygen defect per unit cell and exhibit a good oxygen ion conduction properties $\left[{ }^{41}\right]$.

\subsubsection{Cathode}

On the cathode side, diatomic oxygen molecules are reduced to oxygen ions by accepting electrons from the external circuit and produced oxygen ions are taken into oxygen vacancy defect in the electrolyte. Therefore, these electrochemically active steps are restricted to an area close to triple phase boundaries. Mixed ionic and electronic conductors (MIEC) offering more TPBs areas gradually attract the considerable attention in the development of cathode materials. The investigation of oxygen reduction reaction mechanisms in the MIEC cathode have indicated that the elementary reactions in different interface and bulk are usually considered to separate as follows $\left[{ }^{36,37}\right]$ : (1) the surface reduction of $\mathrm{O}_{2}$ molecules involving adsorption, dissociation and incorporation of the oxygen species into the lattice 
of the cathode materials; (2) ionic transport through the porous cathode toward the electrolyte driven by concentration difference of oxygen vacancy; and (3) the charge transfer of oxygen ions at the cathode/electrolyte interface. A satisfactory cathode material should supply sufficient electron transport $(100 \mathrm{~S} / \mathrm{cm})$, oxygen defect transport $(0.01 \mathrm{~S} / \mathrm{cm})$, oxygen molecule supply (adequate porosity), and high catalytic activity for oxygen reduction reaction in accordance with the oxygen reduction mechanisms. Meanwhile, superior self-chemical stability and excellent thermodynamic and chemical compatibility with adjacent components are expected in the development of cathode materials. Based on the above requirement, various new cathode materials have been developed. Composites of Sr-doped $\mathrm{LaMnO}_{3}(\mathrm{LSM})$ and YSZ are a preferred cathode for high-temperature SOFCs operating at $800-1000^{\circ} \mathrm{C}$. Prominent MIEC materials, such as perovskite $(\mathrm{La}, \mathrm{Sr})(\mathrm{Co}, \mathrm{Fe}) \mathrm{O}_{3}$ $\delta$, layered perovskite $\mathrm{Ln}_{2}(\mathrm{Ni}, \mathrm{Co}, \mathrm{Fe}) \mathrm{O}_{4+\delta}$ and double perovskite $\mathrm{LnBa}(\mathrm{Co}, \mathrm{Fe})_{2} \mathrm{O}_{5+\delta}$ have been employed as cathode materials for IT-SOFCs $\left(500-750^{\circ} \mathrm{C}\right)$. Remarkable initial electrochemical performances have been obtained with these MIEC materials and the cathode polarization resistances decrease to about $0.1 \Omega \mathrm{cm}^{2}$ at $750^{\circ} \mathrm{C}$. However, the stability of cathode performance is worth to be concerned after through different time, temperature, thermal cycling, polarization and operating conditions treatment. Cations segregation on cathode surface and impurities production have frequently been reported in research after long-term operation $\left[{ }^{42-45}\right]$. Moreover, instead of pure electronic conductive materials, more and more mixed ionic and electronic conductors (MIEC) with single, layer or double perovskite crystal structure have been developed and applied as commercial cathode materials $\left[{ }^{46-51}\right]$, which exhibit excellent oxygen bulk transport properties. As a consequence, the internal reactive surface and bulk transport pathway (2PB) are activated because of their high ionic conductivity. More interfaces have been introduced in ORR process, more complexity of reaction mechanism involved. The mechanisms of oxygen reduction and transport are still indistinct in different types of MIEC cathode materials. Especially under polarization conditions, those variation of intrinsic properties are hard to detect due to the lack of advanced techniques operating under electrochemical conditions. The lack of intrinsic properties of materials and over-potential distribution theory under electrochemical conditions limits the understanding of the oxygen reduction reaction 
mechanism and the optimization of electrochemical performance and stability of cathode. Therefore numerous models have been established to simulate the electrode reaction procedures and predict the electrode performance on operation cells based on those intrinsic parameters and well-known ORR mechanism. Therefore, important issues in the field of cathode development are exploring the influence of various factors, especially polarization, to the oxygen reduction reaction mechanism and developing advanced materials, microstructure, or buffer layers to inhibit the segregation and impurities production.

\subsection{Fundamental Background of Solid Oxide Fuel Cells Cathode}

\subsubsection{Cathode Oxygen Reduction Reaction Mechanism Models}

Electrode reaction mechanism is a fundamental issue for electrochemistry study, which reveals how to optimize electrode and performance. Over the past 30 years numerous SOFCs cathode models have been gradually built up by researchers due to the lack of solid state ionic conductivity theories and heterogeneous reaction mechanisms. Modeling and simulation techniques have been used to improve understanding of the reaction mechanisms and kinetics of electrode processes in SOFCs. It has been well accepted that two charge transfer processes occur across the electrode/electrolyte and electrode/current collector interface coupling with over-potential $\left[{ }^{46,52}\right]$. Over-potential relates to the potential difference between a thermodynamically determined standard electrode reduction potential and the experimentally observed electrode potential. The electrochemical activity of these two charge transfer processes generally are expressed by Butler-Volmer equation under electrochemical driving forces. The bulk oxygen transports in electrode or electrolyte obey Fick's law and Nernst-Planck equation, either based on constant electric field or local charge neutrality.

The most arguable issue for micro-models is whether the surface oxygen reduction reaction involves an electrochemical process and multiple intermediate species. Adler $\left[{ }^{52,53}\right]$, as one of the early researchers on ORR mechanisms, claimed the oxygen reduction reaction is a purely chemical process at the gas/electrode interface and charge transfer processes occur 
through the electrode/electrolyte and electrode/current collector interface. The different processes can response to the various cell impedances. With further research, in view of complexity of oxygen reduction reaction, some researchers proposed that oxygen reduction needs to be finished in many sequential steps and products some chargeable intermediates during the reaction process, instead of a single chemical step. And since oxygen reduction is consist of chargeable particles involved elementary reactions, it has been believed that the cathode oxygen reduction reaction of SOFCs was an electrochemical process $\left.{ }^{54-60}\right]$. Various elementary reactions were offered by researchers based on the overall oxygen reduction reaction:

$$
{ }_{z}^{1} O(\text { gas })+\left\{V_{o}^{\bullet \bullet}+2 e^{\prime}\right\}(\text { MIEC / Electrolyte }) \Leftrightarrow O_{o}^{\times}(\text {MIEC / Electrolyte })
$$

Adsorbed oxygen species, $\mathrm{O}^{-}$and $\mathrm{O}_{2}^{-}$were all possibly appear during one or more steps. F.H. van Henveln assumed that there were three pathways for the charge transfer near 3PB with LSM electrode.

Model 1

$$
\begin{aligned}
& O_{2}(g) \Leftrightarrow 2 O_{a d} \\
& O_{a d}+e^{\prime} \Leftrightarrow O_{a d}^{-} \\
& O_{a d}^{-} \Leftrightarrow O_{T P B}^{-} \\
& O_{T P B}^{-}+e^{\prime}+V_{O}^{\bullet \bullet} \Leftrightarrow O_{O}^{\times}
\end{aligned}
$$

Model 2

$$
\begin{aligned}
& O_{2}(g)+e^{\prime} \Leftrightarrow O_{2, a d}^{-} \\
& O_{2, a d}^{-}+e^{\prime} \Leftrightarrow 2 O_{a d}^{-} \\
& O_{a d}^{-} \Leftrightarrow O_{T P B}^{-} \\
& O_{T P B}^{-}+e^{\prime}+V_{O}^{\bullet \bullet} \Leftrightarrow O_{O}^{\times}
\end{aligned}
$$

Model 3

$$
\begin{aligned}
& O_{2}(g)+e^{\prime} \Leftrightarrow O_{2, a d}^{-} \\
& O_{2, a d}^{-} \Leftrightarrow O^{-} \\
& O_{2, T P B}^{-}+e^{\prime}+V_{o}^{\bullet \bullet} \Leftrightarrow O_{O}^{\times}+O_{a d} \\
& O_{a d}+e^{\prime}+V_{O}^{\bullet \bullet} \Leftrightarrow O_{O}^{\times}
\end{aligned}
$$


Liu et al. proposed several possible reaction pathway for the reaction on gas/MIEC interface. Firstly only $\mathrm{O}^{-}$was considered as charged intermediates in oxygen reduction,

Model 4

$$
\begin{aligned}
& \frac{1}{2} O_{2, g a s} \Leftrightarrow \frac{1}{2} O_{2, a d} \\
& \frac{1}{2} O_{2, a d} \Leftrightarrow O_{a d} \\
& O_{a d}+e^{\prime} \Leftrightarrow O_{a d}^{-} \\
& O_{a d}^{-}+e^{\prime}+V_{O}^{\cdot \bullet^{\circ}} \Leftrightarrow O_{O}^{\times}
\end{aligned}
$$

Model 5

$$
\begin{aligned}
& \frac{1}{2} O_{2, g a s} \Leftrightarrow \frac{1}{2} O_{2, a d} \\
& \frac{1}{2} O_{2, a d}+e^{\prime} \Leftrightarrow O_{2, a d}^{-} \\
& O_{2, a d}^{-}+e^{\prime} \Leftrightarrow 2 O_{a d}^{-} \\
& O_{a d}^{-}+e^{\prime}+V_{o}^{\bullet \bullet} \Leftrightarrow O_{O}^{\times}
\end{aligned}
$$

Or, both $\mathrm{O}^{-}$and $\mathrm{O}_{2}^{-}$were involved in the reaction:

Model 6

$$
\begin{aligned}
& \frac{1}{2} O_{2, g a s} \Leftrightarrow \frac{1}{2} O_{2, a d} \\
& \frac{1}{2} O_{2, a d} \Leftrightarrow O_{a d} \\
& O_{a d}+e^{\prime} \Leftrightarrow O_{a d}^{-} \\
& O_{a d}^{-}+e^{\prime} \Leftrightarrow O_{a d}^{2-} \\
& O_{a d}^{2-}+V_{O}^{\bullet \bullet} \Leftrightarrow O_{O}^{\times}
\end{aligned}
$$

Model 7

$$
\begin{aligned}
& \frac{1}{2} O_{2, g a s} \Leftrightarrow \frac{1}{2} O_{2, a d} \\
& \frac{1}{-} O+e^{\prime} \Leftrightarrow{ }_{-}^{1} O^{-} \\
& 2^{2, a d} \\
& \frac{1}{2} O^{-}+e^{2, a d} \\
& 2^{2, a d} O^{-} \\
& O_{a d}^{-}+e^{\prime} \Leftrightarrow O_{a d}^{2-} \\
& O_{a d}^{2-}+V_{O}^{\bullet \bullet} \Leftrightarrow O_{O}^{\times}
\end{aligned}
$$


Further, they discussed the instantaneous relationship of intermediate species that is influenced by kinetics more than thermodynamics. Hence the reaction confirmed as electrochemical. However, based on present available techniques, it is still hard to determine the exact nature of intermediate species in the reaction.

Meanwhile, since researchers considered that cathode reaction as an electrochemical process, surface over-potential needs to be introduced into modeling simulations except for the single electrode/electrolyte interface over-potential, which results from the adsorbed chargeable intermediates. The distributions and relationship of those potentials (interface over-potential and surface over-potential) and resultant influence on electrode processes are complex and still unclear in current investigation. J. Fleig $\left[{ }^{56}\right]$ discussed the application of surface over-potential change $\Delta \chi$ under electron transfer step control and ion transfer step control conditions. He also studied the relationship of MCE surface over-potential $(\Delta \chi)$ and electrode/electrolyte interface over-potential $(\eta)$. Liu $\left[{ }^{61}\right]$ developed a 2-D model based on Fleig's theory of MIEC surface over-potential. The simulation results show that under low over-potential the ionic transportation will be the rate limiting step which means low overpotential doesn't strongly affect oxygen reduction on the surface. Under high over-potential $(750 \mathrm{mV})$, the sheet resistance will be the main factor of the reaction rate which means that the surface exchange step will be the rate limiting step. In MIEC bulk, based on high electronic and ionic conductivity, the uniform electrochemical potentials are assumed in many models. However, the assumption of uniform Fermi-level and perfect current collection throughout the cathode might be problematic for the systems with high oxygen vacancy concentration and certain thin-film structure. And the influence of over-potential on the bulk pathway were discussed based on drift diffusion equations by our group. Therefore, besides the thermodynamic concentration gradient, electrical field force produced by the electrostatic potential is also the driving forces in the ion diffusion and introduces new concepts in handling chemical and electrical contributions in the overall electrochemical response of electrode system. But because of the lack of technology, the detection of single surface over-potential and distinction between different over-potential is hard to perform to prove the theoretical analysis. Therefore, how surface over-potential works on surface molecular diffusion and exchange processes, how interface over-potential 
and surface over-potential influence with each other and how electrostatic potential distributes in bulk between surface and interface area are still the open questions in the development of cathodes for IT-SOFCs. It is found that the electrode bulk is not locally electroneutral and the resultant electrostatic gradient can be established in the region near electrolyte due to the uneven distributions of ions and electrons.

\subsubsection{Cathode Materials}

Three types series of ceramic oxide that have been studied as cathode materials in the past 30 years are perovskites $\mathrm{ABO}_{3-\delta}$, layered perovskites $\mathrm{A}_{2} \mathrm{BO}_{4+\delta}$, and ordered double perovskites $\mathrm{AA}^{\prime} \mathrm{B}_{2} \mathrm{O}_{5+\delta}$ as below.

\subsubsection{1 $\mathrm{ABO}_{3}$ Perovskite Oxides}

A significant number of oxide compositions with the $\mathrm{ABO}_{3-\delta}$ perovskite structure have been investigated as cathode materials in IT-SOFCs. A perovskite-type oxide has the general formula $\mathrm{ABO}_{3}$, in which $\mathrm{A}$ and $\mathrm{B}$ are cations with a total charge of +6 . The A-site cation is a mixture of the rare and alkaline earth (such as $\mathrm{La}$ and $\mathrm{Sr}, \mathrm{Ca}$ or $\mathrm{Ba}$ ), while the Bsite cation is a reducible transition metal such as $\mathrm{Mn}, \mathrm{Fe}, \mathrm{Co}$ or Ni. The larger A-site cations are coordinated to twelve oxygen anions while the B cations occupy the much smaller room and are coordinated to six oxygen anions. Fig. 2.2 shows the typical structure of the cubic perovskite $\mathrm{ABO}_{3-\delta}$. The $\delta$ oxygen vacancies are formed along with divalent alkaline mixture of A-site and the valence alternation of B-site transition metal based on charge compensation principle. Vast oxygen defects and valence alteration in cubic perovskites phase generate ionic and electronic conductivity to promote oxygen reduction activity on cathode surface. Full or partial substitution of A or B cations with cations of different valence to further design and optimize the cathode materials [ $\left.{ }^{62,63}\right]$. However, the distortion of $\mathrm{BO}_{6}$ octahedral occurs in many doped perovskite materials without cubic symmetry due to the ionic radius of the A and B substituted atoms, which impacts the superior mixed ionic and electronic conduction properties. The degree of distortion or tilt in $\mathrm{ABO}_{3}$ perovskites can be determined in term of the Goldschmidt tolerance factor ( $\mathrm{t}$ ) as follows: 


$$
t=\left(r_{A}+r_{B}\right) / \sqrt{2}\left(r_{B}+r_{O}\right)
$$

Where $r_{\mathrm{A}}, r_{\mathrm{B}}$ and $r_{\mathrm{O}}$ are the effective ionic radii of $\mathrm{A}, \mathrm{B}$ and $\mathrm{O}$ ions, respectively. When the tolerance factor is near unity, an ideal cubic structure can be obtained. The introduction of smaller A or bigger B cations reduce the tolerance factor with a cation displacement and tilting of $\mathrm{BO}_{6}$ octahedral and transfer phase structure to an orthorhombic symmetry. Therefore, with a rational selection of A- and B- site cations, a large and stable number of oxygen ion vacancies can be introduced into cathode materials facilitating surface oxygen reduction and bulk ionic oxygen transport $\left.{ }^{62,64-71}\right]$. Meanwhile, various investigation on doped lanthanum ferrites/cobaltite, it indicates that the enhancement of ionic conductivity is heavily influenced by $\mathrm{Sr}$ concentration at the A-site while the electronic conductivities is impacted by $\mathrm{Fe}$ and $\mathrm{Co}$ concentration at the B-site $\left[{ }^{50,54,72-77}\right]$. The A-site deficiency has only a small effect on the CTE while much more $\mathrm{Sr}$ content results in a higher CTE due to higher oxygen vacancy concentrations.

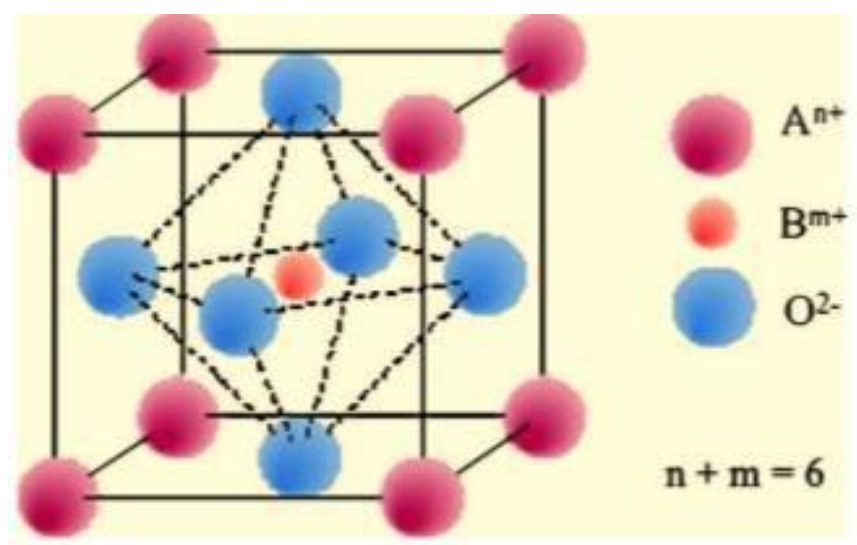

Figure 2.2 Structure of simple cubic perovskite

Strontium-doped lanthanum manganite (LSM) is a common perovskite materials used as cathode in SOFCs. It remains the preferred choice for high-temperature SOFCs in the 800$1000^{\circ} \mathrm{C}$ range because of its high electrical conductivity and high catalytic activity for oxygen reduction reaction. Moreover, LSM shows excellent stability for long-term performance due to high thermal stability, and chemical compatibility with standard 
electrolytes at operating conditions. Researchers continue to improve its performance at intermediate temperatures $\left(500-750^{\circ} \mathrm{C}\right)\left[{ }^{19,78-84}\right]$.

Perovskite-related cobaltites have high mixed electronic and ionic conductivities and exceptional electrochemical activity for oxygen reduction and possess considerably better cathodic and transport properties [ $\left.{ }^{51,85-89}\right]$. As for the manganite and ferrite electrodes, the primary attention for potential electrochemical applications is drawn to perovskite-type $(\mathrm{Ln}, \mathrm{Sr}) \mathrm{CoO}_{3-8}$ and solid solutions on their base. $\mathrm{LaCoO}_{3}$ exhibits a rather high electronic density of states near the Fermi level. The marked catalytic properties of $\mathrm{LaCoO}_{3}$ are associated with electron occupation of the filled $d$ state near $\mathrm{E}_{\mathrm{F}}$ and with the buildup of surface charge so as to enhance the electron transfer between a surface cation and a potentially catalyzed species $\left[{ }^{90-93}\right]$. $\mathrm{La}_{1-\mathrm{x}} \mathrm{Sr}_{\mathrm{x}} \mathrm{CoO}_{3-\delta}$ exhibits a complex behavior with respect to the dependence of non-stoichiometry, electrical, and magnetic properties on the strontium content, temperature. Sr-doped samarium cobaltite $\left(\mathrm{Sm}_{1-\mathrm{x}} \mathrm{Sr}_{\mathrm{x}} \mathrm{CoO}_{3}, \mathrm{SSC}\right)$ is another widely studied cathode material for IT-SOFCs showing a higher electrical conductivity. The electrical conductivity of this composition shows metallic-like behavior and reaches up to $10^{3} \mathrm{~S} / \mathrm{cm}$ in the temperature range of $800-1100^{\circ} \mathrm{C}\left[{ }^{94-97}\right]$. Unfortunately, they typically have high CTE $\left(>20 \times 10^{-6} \mathrm{~K}^{-1}\right)$ as a result of the formation of oxygen vacancies, spin-state transitions associated with $\mathrm{Co}^{3+}$, and the relatively weaker $\mathrm{Co}-\mathrm{O}$ bond compared with the Mn-O bond. Therefore, modification of the composition has been studied to mitigate the effects of thermal expansion. Moreover, undesirable interfacial reaction between the electrode and YSZ electrolyte forms an insulating phase at low temperatures $\left.\left(700^{\circ} \mathrm{C}\right){ }^{[98}\right]$. The utilization of perovskite-related cobaltite requires an additional intermediate barrier layer, typically of doped ceria, to prevent the formation of low conductive impurities without negatively effects on the electrochemical performance. Lanthanum ferrite is expected to be more stable than cobaltite perovskites due to the stable electronic configuration $3 \mathrm{~d}^{5}$ of $\mathrm{Fe}^{3+}$ ion $\left[{ }^{99}\right]$. Sr-doped $\mathrm{LaFeO}_{3}$ (LSF) cathodes have shown promising performance concerning the power density and stability at $750^{\circ} \mathrm{C}$. In iron-based perovskite cathodes, TECs are relatively comparable with those of the YSZ and GDC electrolyte. In addition, reactivity with YSZ is remarkably reduced [100-106]. By incorporating $\mathrm{La}$ deficiency in $\mathrm{La}_{0.8} \mathrm{Sr}_{0.2} \mathrm{FeO}_{3}$, Ralph et al. $\left[{ }^{99}\right]$ found that the area specific 
resistance (ASR) of this cathode was significantly reduced and reached $0.1 \Omega \mathrm{cm}^{2}$ at $800^{\circ} \mathrm{C}$. No significant degradation over $500 \mathrm{~h}$ operation indicates A-site deficient $\mathrm{La}_{0.8} \mathrm{Sr}_{0.2} \mathrm{FeO}_{3}$ is a potential cathode material for lower-temperature SOFCs.

Combining the extraordinary catalysis of cobaltite with the stability of ferrite, $\mathrm{La}_{1-}$ ${ }_{x} \mathrm{Sr}_{\mathrm{x}} \mathrm{Co}_{\mathrm{y}} \mathrm{Fe}_{1-\mathrm{y}} \mathrm{O}_{3-\delta}(\mathrm{LSCF})$ have been widely investigated in the series of perovskite materials. Remarkable electronic (300 S/cm)and ionic conductivity $(0.01 \mathrm{~S} / \mathrm{cm})$ and catalysis have been observed at intermediate temperature $600-800^{\circ} \mathrm{C}\left[{ }^{107-111}\right]$. The oxygen self-diffusion coefficient of LSCF is $2.6 \times 10^{-9} \mathrm{~cm}^{2} / \mathrm{s}$ at $500^{\circ} \mathrm{C}$, superior to that of LSM with $10^{-12} \mathrm{~cm}^{2} / \mathrm{s}$ at $1000^{\circ} \mathrm{C}$. In addition, the TECs is $13.8 \times 10^{-6} \mathrm{~K}^{-1}$ for $\mathrm{La}_{0.6} \mathrm{Sr}_{0.2} \mathrm{Co}_{0.2} \mathrm{Fe}_{0.8} \mathrm{O}_{3-\delta}$ at $700^{\circ} \mathrm{C}$, which matches with commonly used electrolytes $\left[{ }^{112-114}\right]$. Mai et al. $\left[{ }^{115}\right]$ recently observed that a small A-site deficiency and a high strontium had a particularly positive effect on the cell performance using $\mathrm{La}_{1-\mathrm{x}-\mathrm{y}} \mathrm{Sr}_{\mathrm{x}} \mathrm{Co}_{0.2} \mathrm{Fe}_{0.8} \mathrm{O}_{3-\delta}$ as a cathode material. The measured current densities of cells consisting of $\mathrm{La}_{1-x-y} \mathrm{Sr}_{\mathrm{x}} \mathrm{Co}_{0.2} \mathrm{Fe}_{0.8} \mathrm{O}_{3-\delta}$ cathode, GDC electrolyte, and a NiOYSZ anode were as high as $1.76 \mathrm{~A} / \mathrm{cm}^{2}$ at $800^{\circ} \mathrm{C}$ and $0.7 \mathrm{~V}$, which is about twice the current density of cells with LSM/YSZ cathodes.

However, long-term degradation of strontium-doped perovskite cathode materials remains a concern for this class of materials due to Sr out-diffusion on the cathode surface. As a result, less effective reaction areas and more secondary insulating phase formation suppress the overall oxygen reduction reaction activity. It has been found that partial decomposition of the perovskite usually has severely influence on the cell degradation than interface reactions during the operation $\left[{ }^{116-123}\right]$. The improvement of cathode long-term performance is one of the most important issues for the development of IT-SOFCs.

\subsubsection{Layered-Perovskite Oxides}

Recently, many investigation on $\mathrm{A}_{2} \mathrm{BO}_{4}(\mathrm{~A}=\mathrm{La}, \mathrm{Pr}$ and $\mathrm{Nd}, \mathrm{B}=\mathrm{Co}, \mathrm{Fe}$ and Ni) oxides with the $\mathrm{K}_{2} \mathrm{NiF}_{4}$-type structure were reported as a promising candidate of cathode materials for IT-SOFCs $\left[{ }^{124-132}\right]$. The ideal $\mathrm{K}_{2} \mathrm{NiF}_{4}$-type structure can be regarded as a combination of the $\mathrm{ABO}_{3}$ perovskite and $\mathrm{Ln}_{2} \mathrm{O}_{2}$ rock-salt layers stacked one upon the other along the c-axis as shown in Fig. 2.3. This structure has been demonstrated to be able to accommodate a significant interstitial oxygen (in the center of lanthanum tetrahedron) or generate lots of oxygen vacancies (in perovskite layer) with proper doping strategy. The two types of non- 
stoichiometry oxygen enable the alteration of the physical-chemical properties in a wide range. Previous studies $\left[{ }^{85,97,100,113}\right]$ have shown that rapid oxygen diffusion, better thermochemical stability and thermal expansion coefficient have been evidenced in $\mathrm{A}_{2} \mathrm{BO}_{4}$ oxides compared with perovskite $\mathrm{ABO}_{3}$ oxides.

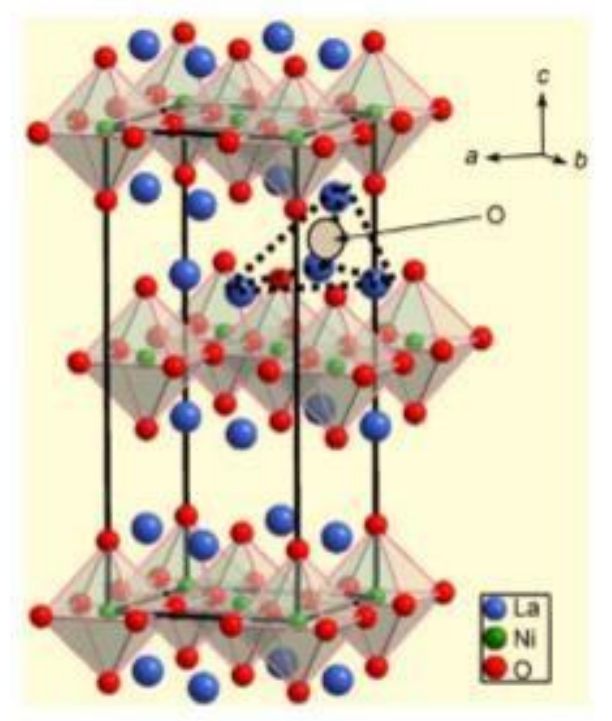

Figure 2.3 Structure of layered perovskite

A collection of surface oxygen exchange coefficients and bulk oxygen diffusion coefficients of different $\mathrm{A}_{2} \mathrm{BO}_{4+\delta}$ indicates that the layered perovskite have relatively higher bulk oxygen diffusion properties than LSCF. The substitution of A-site cations by divalence element has negative effect on oxygen diffusion, which reduces the amount of interstitial oxygen defects and results in the decrease of the oxygen diffusion coefficient, but it has no significant effect on surface oxygen exchange performance $\left[{ }^{129,133}\right]$. Whereas B-site doping has greatly different impact on $k$ and $D$ values, depending on the intrinsic properties of doping element, as that observed before in the perovskite materials $\left[{ }^{134-136}\right]$. In general, substituting nickel with cobalt dramatically enhances both $k$ and $D$ due to larger oxygen non-stoichiometry generation and high catalytic activity of cobalt. Further investigation on the $k$ coefficients for $\mathrm{La}_{2-\mathrm{x}} \mathrm{Sr}_{\mathrm{x}} \mathrm{Ni}_{1-\mathrm{y}} \mathrm{M}_{\mathrm{y}} \mathrm{O}_{4}$ compounds $(\mathrm{M}=\mathrm{Fe}, \mathrm{Cu}, \mathrm{Co})$ indicated that substituting copper for nickel seems to reduce $k$ values and substitution of iron, at least for small amounts, seems to have slightly influence on the catalytic activity. 
Compared to the widespread $\mathrm{Sr}$ and Co co-doped perovskite cathodes $(\mathrm{LaSr})(\mathrm{CoFe}) \mathrm{O}_{3-\delta}$ (LSCF), $\mathrm{La}_{2} \mathrm{NiO}_{4+\delta}(\mathrm{LNO})$ displays smaller thermal expansion coefficient making it more compatible with intermediate temperature electrolytes such as GDC and LSGM, and thus enhances the long-term stability $\left[{ }^{133,137-139}\right]$. It has been shown that substitutions at the A site by $\mathrm{Sr}$ or at the $\mathrm{B}$ site of $\mathrm{A}_{2} \mathrm{BO}_{4}$ compounds influence the amount of oxygen stoichiometry, which in turn effects on the thermal expansion properties $\left[{ }^{140-142}\right]$. Most of $\mathrm{A}_{2} \mathrm{BO}_{4+\delta}$ compounds exhibit lower conductivities ranging from 40 to $100 \mathrm{~S} / \mathrm{cm}$ at 400 $800^{\circ} \mathrm{C}$ than the well-known perovskite materials with p-type conducting behavior. By comparing the total electronic conductivity of different Sr-doped Ln2MO4 ( $\mathrm{M}=\mathrm{Ni}, \mathrm{Co}, \mathrm{Fe}$, $\mathrm{Mn})$ materials, it can be found that $\mathrm{Ln}_{2} \mathrm{NiO}_{4+\delta}$ always have the highest conductivity and the lowest activation energy [ $\left.{ }^{143}\right] . \mathrm{Ln}_{2} \mathrm{CoO}_{4+\delta}$ compounds achieve comparable conductivity values with the analogues nickelates above $600^{\circ} \mathrm{C} . \mathrm{Ln}_{2}(\mathrm{Fe}, \mathrm{Mn}) \mathrm{O}_{4+\delta}$ oxides exhibits relatively low conductivity, less than $20 \mathrm{~S} / \mathrm{cm}$ at $600^{\circ} \mathrm{C}$ in air. By further improving the total conductivity of different $\mathrm{A}_{2} \mathrm{BO}_{4+\delta}$ compounds, di-valent cations, such as $\mathrm{Sr}$, have been doped into oxides considering the different electro-neutrality compensation mechanisms. It can be found $\mathrm{Sr}^{2+}$ doping seems to have great effect on the conductivity. The ferrites doped with $1.2 \mathrm{~mol} \mathrm{Sr}^{2+}$ show the highest conductivity values among the $\mathrm{La}_{2}-\mathrm{Sr}_{x} \mathrm{FeO}_{4}$. Substitution of strontium in $\mathrm{Ln}_{2-\mathrm{x}} \mathrm{Sr}_{\mathrm{x}} \mathrm{M}_{1-\mathrm{y}} \mathrm{M}^{\prime}{ }_{\mathrm{y}} \mathrm{O}_{4}\left(\mathrm{Ln}=\mathrm{La}, \mathrm{Nd} ; \mathrm{M}, \mathrm{M}^{\prime}=\mathrm{Cu}, \mathrm{Fe}, \mathrm{Ni}, \mathrm{Co}\right.$ ) has effectively retarded chemical reactivity with GDC and SDC electrolyte at high temperature $\left[{ }^{143-147}\right]$. The investigation indicated that $\mathrm{Sr}$-doped $\mathrm{Ln}_{2} \mathrm{MO}_{4}$ exhibited remarkable chemical compatibility. No reaction was observed at $1000^{\circ} \mathrm{C}$ in air, even for a prolonged $144 \mathrm{~h}$ sintering duration. Sr- doped $\mathrm{Pr}_{2} \mathrm{MO}_{4}$ have also been studied. The result shows that the addition of Sr has dramatically improved thermo-stability and chemical compatibility of $\mathrm{A}_{2} \mathrm{BO}_{4+\delta}$ with YSZ electrolyte at high temperature. All above information indicated that it is the situation of $\mathrm{Sr}$ and Co doping in $\mathrm{Ln}_{2} \mathrm{NiO}_{4}$.

\subsubsection{3 $\mathrm{AA}^{\prime} \mathrm{B}_{2} \mathrm{O}_{5}$ Double Perovskite Oxides}

In the mixed conducting oxides with the general formula $\mathrm{AA}^{\prime} \mathrm{Co}_{2} \mathrm{O}_{5+\delta}(\mathrm{A}=$ rare earths, $\mathrm{Y}$ and $\mathrm{A}^{\prime}=\mathrm{Ba}, \mathrm{Sr}$ ), the combination of rare and alkaline earths on the A-site gives rise to the existence of A site ordering. The ideal structure of this family of compounds can be 
represented by the stacking sequence $\mathrm{LnO} \delta-\mathrm{CoO}_{2}-\mathrm{BaO}-\mathrm{CoO}_{2}$. The structures of representative examples of this class of compounds are shown in Fig. 2.4. Transformation of a simple cubic perovskite with randomly A-sites occupation into a overlapped crystal structure with alternating lanthanide oxide and alkali earth oxide planes reduces the strength of oxygen binding and tenders disorder-free channels for ionic transport, thereby theoretically improving the oxygen diffusivity by many orders of magnitude $\left[{ }^{148-156}\right]$.

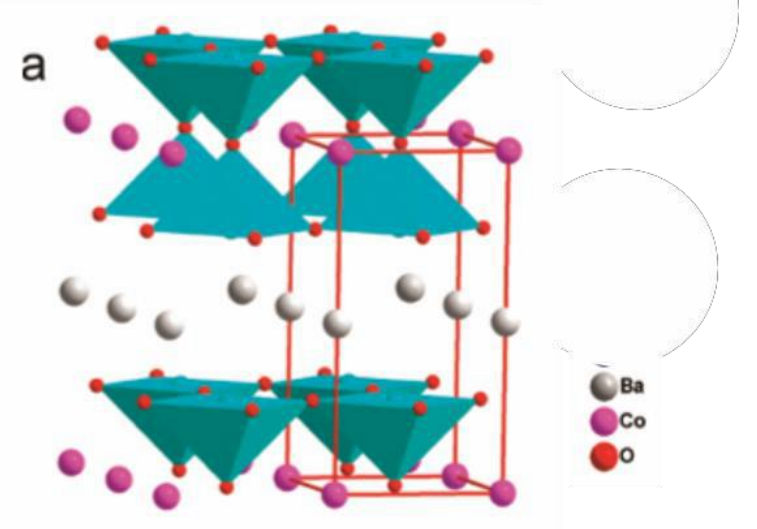

Figure 2.4 Structure of double-layered perovskite

Taskin et al. $\left[{ }^{157}\right]$ have observed high rates of oxygen incorporation in $\mathrm{GdBaB}_{2} \mathrm{O}_{5+\delta}(\mathrm{B}=\mathrm{Mn}$, $\mathrm{Co}$ ), indicating the importance of vacancy layer on this process. Oxygen diffusivity could be enhanced by several orders of magnitude by eliciting crystallographic ordering of lanthanide and alkali-earth oxide layers in the A-site sub-lattice. Zhang et al. $\left.{ }^{158}\right]$ found that the ionic radius of $\mathrm{A}$ site lanthanide cations in $\mathrm{LnBaCo}_{2} \mathrm{O}_{5+\delta}$ is directly related to the stability of ordered structure under reduction condition. The ordered double perovskite could be kept for the composition with $\mathrm{Ln}=\mathrm{Pr}, \mathrm{Nd}, \mathrm{Sm}$ and $\mathrm{Gd}$ but not for $\mathrm{Ln}=\mathrm{La}$ or $\mathrm{Y}$. Rapid oxygen diffusion, exceeding $10^{-5} \mathrm{~cm}^{2} / \mathrm{s}$ at $600^{\circ} \mathrm{C}$, and high surface exchange rates have been yielded with quite low activation energy, and the ionic conductivity of about $0.01 \mathrm{~S} / \mathrm{cm}$ could be achieved at nearly $500^{\circ} \mathrm{C}$ as well. Tarancon et al. $\left[{ }^{154,159}\right]$ have investigated the intrinsic oxygen transport and exchange of ceramic $\mathrm{GdBaCo}_{2} \mathrm{O}_{5+\delta}$ by IEDP/SIMS method, and achieved suitable tracer oxygen diffusion coefficient and oxygen surface exchange coefficient with low activation energies compared to other cobaltite such 
as LSC and LSCF. These compounds showed superior electrode performances with SDC electrolyte, especially $\mathrm{PrBaCo}_{2} \mathrm{O}_{5+\delta}$ which possessed an ASR of $0.213 \Omega \mathrm{cm}^{-2}$ at $600^{\circ} \mathrm{C}$. Moreover, excellent full cell performance with $\mathrm{PrBaCo}_{2} \mathrm{O}_{5+\delta}$ cathode and SDC electrolyte have been observed, showing considerable maximum power density $600 \mathrm{mWcm}^{-2}$ at $600^{\circ} \mathrm{C}$. All the properties mentioned above show that ordered double perovskite have a promising development potential as MIEC cathode materials for low-temperature SOFCs $\left(\leq 600^{\circ} \mathrm{C}\right)$. Nevertheless, as usually observed in cobaltite cathodes, $\mathrm{LnBaCo}_{2} \mathrm{O}_{5+\delta}$ also shows large thermal expansion coefficient $\left(16.0-20.0 \times 10^{-6} \mathrm{~K}^{-1}\right)$, considerably higher than those of typical electrolytes such as YSZ, GDC and LSGM, giving rise to delamination and cracking during cells fabrication and thermal cycling of operation process. The overlap of characteristic x-ray diffraction peaks increases the level of challenge to distinguish ordered double perovskite from perovskite structure. Therefore, composite electrodes with an incorporation of electrolyte materials were investigated to reduce the thermal mismatch and extend the TPB. Moreover, another feasible thought is the B-site substitution of Co by other transition metals, such as $\mathrm{Ni}, \mathrm{Fe}$ or $\mathrm{Cu}$, to lower TEC and optimize the electrochemical performance. Advanced characterization techniques need to be developed to identify cations ordering arrangement.

\subsubsection{Long-term Stability of Sr-doped Perovskite Cathode}

The successful application of strontium doped perovskites (LSC, LSM, LSCF and SSC) as a mixed ionic and electronic conducting electrode in SOFCs stems from its tolerance of numerous oxygen defects by introduction of di-valence $\mathrm{Sr}$ cations at operating temperatures. However, long-term performance degradation remains a concern for this class of materials. Dense and polished strontium doped perovskite samples were examined to clearly identify surface morphology variation and surface phase and chemical composition alternation $\left[{ }^{118}\right]$. Surface phase structure and chemical composition of strontium doped perovskite cathode materials were investigated after long-term treatment by Auger electron spectroscopy (AES) and transmission electron microscopy (TEM). The detailed nature of the Sr-excess is discussed by means of depth and take-off angle dependent XPS spectra, in combination with chemical and thermal treatments. The degradation of cathode performance is related to 
Sr enrichment and subsequent formation of SrO surface precipitates. As a result, the concentration of B-site transition metal was found to substantially decrease in the outermost surface layers, which generally believed in playing a critical role for catalytic activity of $\mathrm{ABO}_{3}$ perovskite materials. Moreover, the interactions of gaseous/electrode and electrode/electrolyte heterogeneous are seriously impacted by alternated surface composition and structure. Consequently, the formation of strontium-enriched layers on LSCF surfaces is a potential degradation mechanism for LSCF cathode-based cells.

$\mathrm{SrO}$ is electrically insulator and $\mathrm{Sr}$ segregation results in the depletion of B-site transition metals. Consequently, the surface catalytic activity is substantially degraded by the SrO/electrode heterogonous surface structure. Besides surface catalysis, the purity of electrode/YSZ interface is also implicated by $\mathrm{Sr}$ segregation, and the insulator $\mathrm{SrZrO} 3$ is formed inhibiting oxygen ions transfer.

$$
\mathrm{SrO}(s)+\mathrm{ZrO}_{2}(g) \Leftrightarrow \mathrm{SrZrO}_{3}
$$

Meanwhile, the surface strontium layer becomes more basic, reacting with acidic substances, such as the gaseous species $\mathrm{CO}_{2}, \mathrm{SO}_{2}, \mathrm{CrO}_{2}(\mathrm{OH})_{2}, \mathrm{Si}(\mathrm{OH})_{4}$ and $\mathrm{H}_{3} \mathrm{BO}_{3}$. Typical gaseous acid-SrO reactions can be expressed:

$$
\begin{gathered}
\mathrm{SrO}(s)+\mathrm{CO}_{2}(g) \Leftrightarrow \mathrm{SrCO}_{3} \\
\mathrm{SrO}(s)+\mathrm{H}_{2} \mathrm{O}(g) \Leftrightarrow \mathrm{Sr}(\mathrm{OH})_{2}
\end{gathered}
$$

The Sr segregation accelerates the formations of insulating secondary phase measured via X-ray Absorption Spectroscopy (XAS). Another degradation mechanisms related with Sr segregation is deposition and poisoning of chromium main parts of metallic interconnect materials in SOFCs. On the cathode side, $\mathrm{Cr}_{2} \mathrm{O}_{3}$ reacts with oxygen and steam, forming various gaseous acid such as $\mathrm{CrO}_{3}$ and $\mathrm{CrO}_{2}(\mathrm{OH})_{2}$ under the operation temperature of SOFCs. The overall reaction between $\mathrm{Cr}$ involved gaseous acid and segregated $\mathrm{SrO}$ species can be written as follow:

$$
\mathrm{SrO}(s)+\mathrm{CrO}_{3}(\mathrm{~g}) \Leftrightarrow \mathrm{Cr}-\mathrm{Sr}-\mathrm{O} \Leftrightarrow \mathrm{SrCrO}_{4}
$$


This reaction is thermodynamically favored and kinetically rapid. $\mathrm{SrCrO}_{4}$ is insulator and its formation results in reduction of cathode conductivity and porosity, making it harmful to the long term stability of cathode performance.

Thus, the development of Sr-doped perovskite cathode requires an understanding of $\mathrm{Sr}$ surface enrichment behavior. Some workers have suggested that the strontium segregation may be driven by oxygen vacancy concentration variation. Doom et al. found that $\mathrm{Sr}$ segregation at the oxygen-lean side of LSC membranes with a segregation depth of $10 \mathrm{~nm}$ $\left[{ }^{160}\right]$. The concentration of surface strontium has increased with oxygen partial pressure drop and over-potential introduction, suggesting that the surface oxygen vacancy concentration plays a significant role in controlling the degree of segregation. However, Elshof et al. $\left.{ }^{161}\right]$ found that $\mathrm{Sr}$ was enriched within a depth of 60-80 nm on both LSCF membranes side. Lein et al. $\left[{ }^{27}\right]$ demonstrated that $\mathrm{Sr}$ enrichment appeared at the surface that was exposed to the high partial pressure and vanished at the other lower partial pressure side. Another possible driving force of $\mathrm{Sr}$ segregation has been attributed to the uncoordinated ionic radius between doped $\mathrm{Sr}$ and host La cations since the surface $\mathrm{Sr}$ segregation has been observed even without a gradient of oxygen partial pressure. The much larger $\mathrm{Sr}^{2+}$ cation is under a compressive strain state in the bulk resulting in a migration of $\mathrm{Sr}$ towards the surface that can relax materials to a lower energy state. Although various driving forces have been proposed, the detailed mechanisms of $\mathrm{Sr}$ segregation are still an open question. It is imperative to balance these driving forces for the development of new Sr-free cathode materials and modification of the Sr-enriched heterogeneous surface to, enhance the long term stability of SOFCs.

\subsubsection{Hetero-Structured Cathode}

The recently reported rapid oxygen reduction activity at the interface of $(\mathrm{La}, \mathrm{Sr}) \mathrm{CoO}_{3-\delta}$ (LSC113) and ( $\mathrm{La}, \mathrm{Sr})_{2} \mathrm{CoO}_{4}(\mathrm{LSC} 214)$ phases opened up new research questions for the potential role of hetero-structured interfaces in the development of advanced cathodes for solid oxide fuel cells (SOFCs). The investigation have proved that many hetero-structured interfaces exhibit fantastic physical, chemical, and electrochemical properties including enhanced oxygen electro-catalytic activity and electronic/ionic conductivity compared with 
the nature of bulk. As first shown by Sase et al. [ ${ }^{162,163}$, the addition of a RuddlesdenPopper (RP) ( $\left.\mathrm{La} 0.5_{5} \mathrm{Sr}_{0.5}\right)_{2} \mathrm{CoO}_{4+\delta}$ phase on top of the perovskite $\mathrm{La}_{0.8} \mathrm{Sr}_{0.2} \mathrm{CoO}_{3-\delta}$ significantly enhances the surface activity of the oxygen reduction reaction at the heterostructured boundary between the two phases. The secondary ion mass spectrometry (SIMS) with ${ }^{18} \mathrm{O}$ isotope exchange images suggested that a fast oxygen-incorporation paths exists along the hetero-phase boundary of LSC113 and LSC214. And the depth profile ${ }^{18} \mathrm{O}$ of indicated that the surface exchange coefficient, $k$ for the hetero-phase is larger than the value of $k$ for the surface of single phase LSC113 by three orders of magnitude. This effect can enhance the overall rate of $\mathrm{O}_{2}$ reduction on LSCO films by three orders of magnitude compared with that on each phase alone.

A number of investigations have been done to uncover the underlying mechanism of fast ORR kinetics at the LSC113/214 system by using well-defined thin film systems and understand the mechanism of the high catalytic activity of these modified hetero-structured catalysts from a defect chemistry and electronic structure perspective [126,130,164-183 $]$. It shown that the extremely enhancement of electrode performance are strongly attributed to the low valence cations enrichment on the LSC113/214 hetero-structured interface, the anisotropic oxygen incorporation kinetics on the LSC214 and the lattice strain near the LSC113/214 hetero-structured interface.

Density functional theory (DFT) calculations have shown that a large driving force exists in the inter-diffusion of A-site cationic across the hetero-structure interface, stabilizing $\mathrm{Sr}$ in LSC214 bulk. Such inter-diffusion has been postulated to stabilize Sr in LSC214 and La in LSC113 with predicted Sr enrichment near the interface and Sr depletion in LSC113. The strong coupling of Sr enrichment with the increased oxygen vacancy content near the surface and is predicted to result in the reduction of the oxygen vacancy formation energy that may play a direct role in enhancing the oxygen surface exchange or the electronic activation of LSC214 $\left[{ }^{165}\right]$. The coherent Bragg rod analysis method (COBRA) $\left.{ }^{180}\right]$ was applied to study the atomic structure and strontium distribution layer-by-layer in about 6 nm thick LSC214-LSC113 hetero-structure thin films. Significant Sr segregations have been found at the LSC214-LSC113 interface and LSC214 surface. The COBRA results also reveal a significant A-site cationic ordering in the LSC214 phase near to LSC113 side 
that may partially be attributed to the enhancement of catalytic activity of the LSC112/LSC214 hetero-structured thin films. It was also found that a small amount of secondary phase "Sr"-decoration with nanoparticles coverage in the range from $50 \%$ to $80 \%$ of a (001) LSC113 surface improved the surface exchange coefficient, $k$, by an order of magnitude. Based on atomic force microscopy, X-ray diffraction, and X-ray photoelectron spectroscopy results, it suggests that the enhancement with "Sr"-decoration heterostructured surfaces can be attributed largely to catalytically active hetero-structured interface regions between surface Sr-enriched particles and the LSC surface [ ${ }^{168,171-}$ 174,180,183-188]. While, another cationic accumulation have been investigated in the LSC214 phase near to LSC113 edge with lower valence. The reducibility of the Co cation and the valence band offset across LSC112/LSC214 hetero-structured interface were detected by X-ray photoelectron spectroscopy with depth profiling $\left[{ }^{166,167}\right]$. The Co2p core-level photoelectron spectra showed the presence of $\mathrm{Co}^{2+}$ across the LSC113/214 interfaces in both layers. While this is similar to the Co valence state in LSC113 single phase films, it is contrary to the single phase of LSC214 films which reflect the higher oxidation state of cobalt, $\mathrm{Co}^{3+}$ Mostly. The greater reducibility of cobalt across the LSC113/214 heterostructured interface relative to that of Co in the LSC214 single phase film was ascribed to electron donation and transfer of oxygen vacancies from LSC113 across the interfaces, and it is one possible mechanism of enhancement of the oxygen reduction activity at the LSC113/214 hetero-structure $\left[{ }^{167}\right]$.

Another factor that can alter the oxygen reaction activity near a dissimilar interface is the lattice strain induced by the lattice mismatch at the hetero-structured interface [ ${ }^{164,165,175-}$ 179,181,182,189].. Lattice strain exhibits a significant impact on promoting oxygen defect formation, oxygen ion transport and surface adsorption. The surface chemical state and electronic structure of LSC113 have been investigated in both tensilely and compressivelystrained LSC film surfaces. The impact of lattice strain on the surface electronic structure demonstrates that the formation of oxygen vacancy defects in tensile strained LSC113 surface results in a more enhanced electronic density of states (DOS) and indicates a more highly active surface for electron charge transfer in oxygen reduction reaction. Consequently, much faster surface exchange and diffusion properties can be detected in the 
tensilely strained films compared to the compressively strained one. Moreover, the strain can also lead to the reduction of the Co spin transition temperature and Co-O bond strength. The weak Co-O bond strength and enhanced spin moment of the thin films result in a significant enhancement of the oxygen surface exchange kinetics of LSC113 by up to two orders of magnitude. The electrochemical impedance spectroscopy of LNO214 as function of film thickness indicated that increasing volumetric strains in the LNO214 films were correlated with the enhancement of surface exchange kinetics and the reduction of film thickness. Volumetric strains may alter the formation energy of interstitial oxygen in LNO214 films and influence on the surface oxygen exchange kinetics of the LNO214 films. Therefore, surface-decoration of perovskites with heterogeneous interface can strongly affect the oxygen reduction activity, and therefore provide new insights into developing catalysts as SOFC cathode materials with enhanced activity and stability.

\subsection{Infiltration Technique}

Infiltration has been applied extensively in the composite cathode fabrication in recent years based on its unique advantages among many composite techniques. In general, the infiltration technique is used to deposit nanoparticles within a preformed electrode structure. There are several advantages to infiltrated electrodes [ $\left.{ }^{190,191}\right]$ : 1) the nanoparticles provide larger surface area; 2) compared with conventional composite cathode, the infiltrated cathode offer larger contact area between different phases; 3) the low temperature fabrication prevents the reaction between different phases. In recent years, there have been several excellent reviews on SOFC cathode infiltration, mainly from the materials selection point-of-view [ $\left.{ }^{191-193}\right]$.

The oxygen ion conductors were added into perovskite oxides to form a composite cathode which was believed to efficient for improving the cathode performance due to the extension of the active TPB region from the electrolyte/LSM interface to the electrode bulk. The results showed that the infiltration technique was an effective method to enlarge the TPB length compared with conventional composite cathodes [ $\left.{ }^{140,194-201}\right]$.A series of perovskitebased infiltrated cathodes have been studied with different ionic conductors. Cathode symmetric cells prepared by infiltration of LSC into porous YSZ substrates showed a 
polarization resistance of $0.03 \Omega \mathrm{cm}^{2}$ at $700^{\circ} \mathrm{C}\left[{ }^{202}\right]$. The cathode impedances for the LSFYSZ composites were estimated with 40 wt. \% LSF loading at $700^{\circ} \mathrm{C}$, which was approximately $0.1 \Omega \mathrm{cm}^{2}\left[{ }^{190}\right]$. At the same time, the two steps of infiltration allowed separate sintering temperatures for the electrolyte phase and the other components of the electrodes; the porous YSZ scaffold could be sintered at high temperature to ensure the interface connection and MIEC components could be formed at low temperature. This approach was successfully used to fabricate the composite cathodes LSC-YSZ, LSCF-YSZ and LSF-YSZ [ $\left.{ }^{203-208}\right]$. The composition of LSC-YSZ infiltrated cathode was measured followed by sintering LSC in air at different temperature $\left[{ }^{209}\right]$. The formation of LSC perovskite phase was confirmed after calcination at $700^{\circ} \mathrm{C}$. Moreover, since the infiltrated layer was just tens of nanometers, the unique thin heterogeneous interfaces provide new insights into the enhancement of stability and performance due to stress and quantum effect. Recently, Liu et al. found that the stability of LSCF was enhanced, when a dense thin film of LSM was coated on the LSCF surface by infiltration $\left[{ }^{210}\right]$. According to the TEM and EDXS data, it was shown that Co diffused from the LSCF substrate into the interface between LSCF and LSM and made the interface become a hybrid $\operatorname{LSM}(\mathrm{C})$ with properties residing between those of LSM and LSCF which maybe contributed to the long stability of LSCF-based cathode.

Infiltration involves several steps: 1) the preparation of porous electrode scaffold, the porous scaffold is prepared by screen printing or tape casting usually, followed by firing at high temperature to obtain good structural stability and connectivity; 2) the carrier of active materials, either liquid or sol-gel, permeated into the substrate materials and adsorbed onto the surface of particles by capillary force; 3) after reaching equilibrium, the infiltrated electrode is dried and sintered to remove the extra solution and drive the active materials react and deposit on the substrate surface forming nanoscale infiltration phase. As a result, the morphology of the substrate, the composition/ concentration of the infiltration solution and the infiltration process post-treatment, would influence the morphology and properties of infiltrated nanoparticles. In term of the functions of infiltration materials, it's necessary for infiltration nanoparticles to form different morphologies on substrate surface, either isolated island or dense thin film [ $\left.{ }^{140,211-213}\right]$. 
Hence, infiltration is widely used in fabrication of composite cathodes for solid oxide fuel cells. The introduction of nanoparticles of catalytically active, ionically conductive or mixed conductive oxides has provided high rates of oxygen reduction reaction and improved the chemical and thermal stability of composite cathodes. 


\section{Chapter 3: $\quad \mathrm{La}_{2} \mathrm{NiO}_{4+\delta}-$ Infiltrated $\quad\left(\mathrm{La}_{0.6} \mathrm{Sr}_{0.4}\right)_{0.95} \mathrm{Co}_{0.2} \mathrm{Fe}_{0.8} \mathrm{O}_{3-\delta} \quad$ Hetero-structure Cathode}

\subsection{Introduction}

Although a great deal of research has been conducted to develop new cathode materials, for operation at lower temperatures $\left(500-750^{\circ} \mathrm{C}\right), \mathrm{La}_{1-\mathrm{x}} \mathrm{Sr}_{\mathrm{x}} \mathrm{Co}_{1-\mathrm{y}} \mathrm{Fe}_{\mathrm{y}} \mathrm{O}_{3-\delta}(\mathrm{LSCF})$ based cathodes are currently the most popular for solid oxide fuel cells (SOFCs) today. However, main challenges associated with long-term application of LSCF-based cathodes are material instability such as $\mathrm{Sr}$ segregation and surface-limited oxygen reduction reaction (ORR) kinetics. The Sr segregation in LSCF cathode changes the composition stoichiometry and forms inactive surface/interface species (e.g. $\mathrm{SrO}$ ) key issues generating inferior catalytic activity.

In this chapter, a new LSCF-based composite cathode prepared by infiltration is developed, namely $\mathrm{La}_{2} \mathrm{NiO}_{4+\delta}$ (LNO)-infiltrated LSCF. The special interest associated with the employment of LNO materials mainly lies in the following points: 1) Rapid bulk oxygen diffusion and surface oxygen exchange can be offered in the LNO nickelates based on their additional conduction pathway in high concentration of oxygen interstitials. Skinner et al. have confirmed that LNO-based materials show higher bulk oxygen diffusion coefficient D of about $5 \times 10-8 \mathrm{~cm}^{2} \mathrm{~s}^{-1}$ than that of LSCF materials by one order of magnitude, and slightly higher surface oxygen exchange coefficient $\mathrm{k}$ of about $2-5 \times 10^{-7} \mathrm{cms}^{-1}$ at $700-750^{\circ} \mathrm{C}$ ); 2) LNO as $\mathrm{Sr}$ acceptor shows promise by improving cathode stability and electro-catalytic properties without invoking deleterious LSCF surface reactions related to the formation of an inactive $\mathrm{Sr}$ species. Reports have shown there is obvious increase in electronic conductivity and oxygen transfer capability of Sr-containing LNO in comparison to LNO itself. Unlike previous research attempting to physically block Sr-enrichment on the surface of LSCF with thin surface films, a chemical driving force in the LNO/LSCF combination is expected for diffusion of Sr from LSCF into LNO to benefit surface oxygen exchange and material stability of the composite cathode. 3) Hetero-structured interface would potentially extend the active regions of two phase boundary ( $2 \mathrm{~PB}, \mathrm{LSCF} / \mathrm{LNO})$ and triple phase boundary (TPB, LSCF/LNO/gas) with high oxygen transfer, leading to total ORR kinetic 
enhancement of SOFC cathode. Efforts in development of $(\mathrm{La}, \mathrm{Sr}) \mathrm{CoO}_{3-\delta} /(\mathrm{La}, \mathrm{Sr})_{2} \mathrm{CoO}_{4+\delta}$ (LSC113/LSC214) hetero-structured architecture via pulsed laser deposition (PLD) has been made by MTI group. It was found that the LSC214 decoration can enhance the oxygen exchange coefficient $(k)$ of the LSC113 by $\sim 2$ orders of magnitude, and the doubledigit enhancement in the $\mathrm{k}$ was observed for the LSC214-decorated LSCF thin films. The unique interfacial regions were proposed to be responsible for the enhanced ORR kinetics. For these reasons, the main aim of this study is to examine the influence of LNO infiltration into a porous LSCF cathode, on ORR behavior and long-term stability of the LNO-infiltrated LSCF cathodes.

\subsection{Experimental}

The effect of LNO infiltration on electrochemical properties of LSCF cathodes was first investigated by symmetric cells associated with related impedance characterizations. A symmetric cell configuration of LSCF|GDC|YSZ|GDC|LSCF were assembled starting with the preparation of $8 \% \mathrm{Y}_{2} \mathrm{O}_{3}-$ stabilized $\mathrm{ZrO}_{2}$ (YSZ, TZ8Y, Tosoh, Japan) electrolyte supports. Tape casting followed by sintering at $1450^{\circ} \mathrm{C}$ for $5 \mathrm{~h}$ in air was used to form dense YSZ electrolyte pellets with a thickness of about $300 \mu \mathrm{m}$. Identical GDC buffer layers were screen printed symmetrically on both sides of the YSZ electrolyte and then sintered at $1200^{\circ} \mathrm{C}$, to avoid interaction between the YSZ electrolyte and LSCF cathode. Asite deficiency $\left(\mathrm{La}_{0.6} \mathrm{Sr}_{0.4}\right)_{0.95} \mathrm{Co}_{0.2} \mathrm{Fe}_{0.8} \mathrm{O}_{3-\delta}$ (LSCF) powder (Fuel Cell Materials) , was dispersed into an organic vehicle ink (VHE, Fuel Cell Materials) to form a slurry. By subsequent screen printing, porous LSCF layers with a thickness of $20 \mu \mathrm{m}$ were deposited on the GDC buffer layers, Eventually, the bonded layers were co-fired at $1150^{\circ} \mathrm{Cfor} 3 \mathrm{~h}$ to obtain the symmetric cell using LSCF cathode with an active surface area of about $0.7 \mathrm{~cm}^{2}$. For comparison and performance evaluation, LNO was infiltrated into the both sides of LSCF layers in the LSCF $\mid$ GDC $|Y S Z| G D C \mid L S C F$ cell, as described below, to form a symmetric cell using LNO infiltrated LSCF cathode.

The LNO precursor solution with a concentration of $1 \mathrm{~mol} / \mathrm{L}$ was composed of $\mathrm{La}\left(\mathrm{NO}_{3}\right)_{3} \cdot 6 \mathrm{H}_{2} \mathrm{O}$ and $\mathrm{Ni}\left(\mathrm{NO}_{3}\right)_{2} \cdot 6 \mathrm{H}_{2} \mathrm{O}(99.9 \%$, Alfa Aesar) dissolved in a mixture of ethanol and deionized water in a ratio of 1:0.6. We confirmed that an appropriate amount of ethanol 
can promote the formation of LNO phase and improve its distribution status on LSCF substrate. After infiltrating the nitrate solution into each side of porous LSCF cathode, the cells were sintered at $900^{\circ} \mathrm{C}$ for $1 \mathrm{~h}$ to allow the formation of LNO single phase on the LSCF backbone. Repeated infiltration, followed by firing, was carried out to achieve the desired amount of LNO phase in the modified cathode.

Prior to electrochemical characterization, Pt meshes were attached as current collectors to both electrode sides of the symmetric cells by using Pt paste. Electrochemical impedance spectroscopy (EIS) measurements for the symmetrical cells were carried out by an Auto lab station (AUT85484) in ambient air under open circuit voltage (OCV). The frequency range was within 0.01 to $100 \mathrm{kHz}$ at alternating circuit (AC) signal amplitude of $10 \mathrm{mV}$, and the measured temperature was controlled from 550 to $750^{\circ} \mathrm{C}$. All impedance data were collected after holding at the desired temperature for more than $30 \mathrm{~min}$.

For comparison, anode-supported fuel cells using LSCF or LNO-infiltrated LSCF cathodes were prepared and measured to form NiO-YSZ|YSZ|GDC|LSCF and $\mathrm{NiO}-$ YSZ|YSZ|GDC|LNO-infiltrated LSCF. Anode-supported half fuel cells Ni-YSZ/YSZ were commercially available (MSRI, Salt Lake City, UT), consisting of about $10 \mu \mathrm{m}$-thick YSZ electrolyte and $750 \mu$ m-thick Ni-YSZ anode. The GDC buffer layer and bare/modified LSCF cathodes were sequentially applied to the YSZ electrolyte using the same procedures for the fabrication of symmetric cells as mentioned above. The whole cells was then sealed using mica gaskets, attached using Pt meshes with Pt paste for cathode and Ni paste for anode, and installed in a single cell test stand (at the NETL Morgantown, WV site).

The on cell measurements were eventually performed at $750^{\circ} \mathrm{Cwith}$ dry hydrogen as the fuel for anode and ambient air as the oxidant for cathode at each flow rate of $400 \mathrm{sccm}$. A constant current density of $250 \mathrm{~mA} \mathrm{~cm}^{-2}$ was applied to the fuel cells using a DC electronic load to monitor the long-term stability of SOFC cathodes. In addition to the continuous observation of cell voltage, intermittent current-voltage (I-V) curves were also carried out using a Solartron 1287 electrochemical interface combined with a Solartron 1260 frequency response analyzer.

A Panalytical X'pert Pro X-ray diffractometer (XRD) with $\mathrm{Cu} K \alpha$ radiation $(\lambda=1.5406 \AA)$ was used to identify phase composition and crystalline structure. The surface morphology 
analyses for both LSCF and LNO infiltrated LSCF materials were performed using a field emission scanning electronic microscope (FESEM, JEOL 7600F) at an acceleration voltage of $20 \mathrm{kV}$. Transmission electron microscopy (TEM) observations and elemental identifications were also carried out on a JEOL JEM-2100 LaB6 microscope equipped with an energy dispersion spectrometer (EDAX, Evex NanoAnalysis).

3.3Results and Discussion

\subsubsection{Phase Composition}

Fig. 3.1 displays the XRD patterns of LSCF pellets with and without LNO infiltration. All of the XRD peaks of the bare LSCF sample on the electrolyte can be labeled in terms of LSCF, GDC and YSZ phases. While the appearance of single Ruddlesden-Popper (RP) phase for LNO infiltrated LSCF sample confirms the existence of LNO in the LSCF backbone. The diffraction peaks of $\mathrm{LNO}$ phase can be identified as tetragonal $\mathrm{K}_{2} \mathrm{NiF}_{4}$ structure of I4/mmm space group (JCPDS card No. 34-0314), which shows excellent agreement with those reported in related literature. 


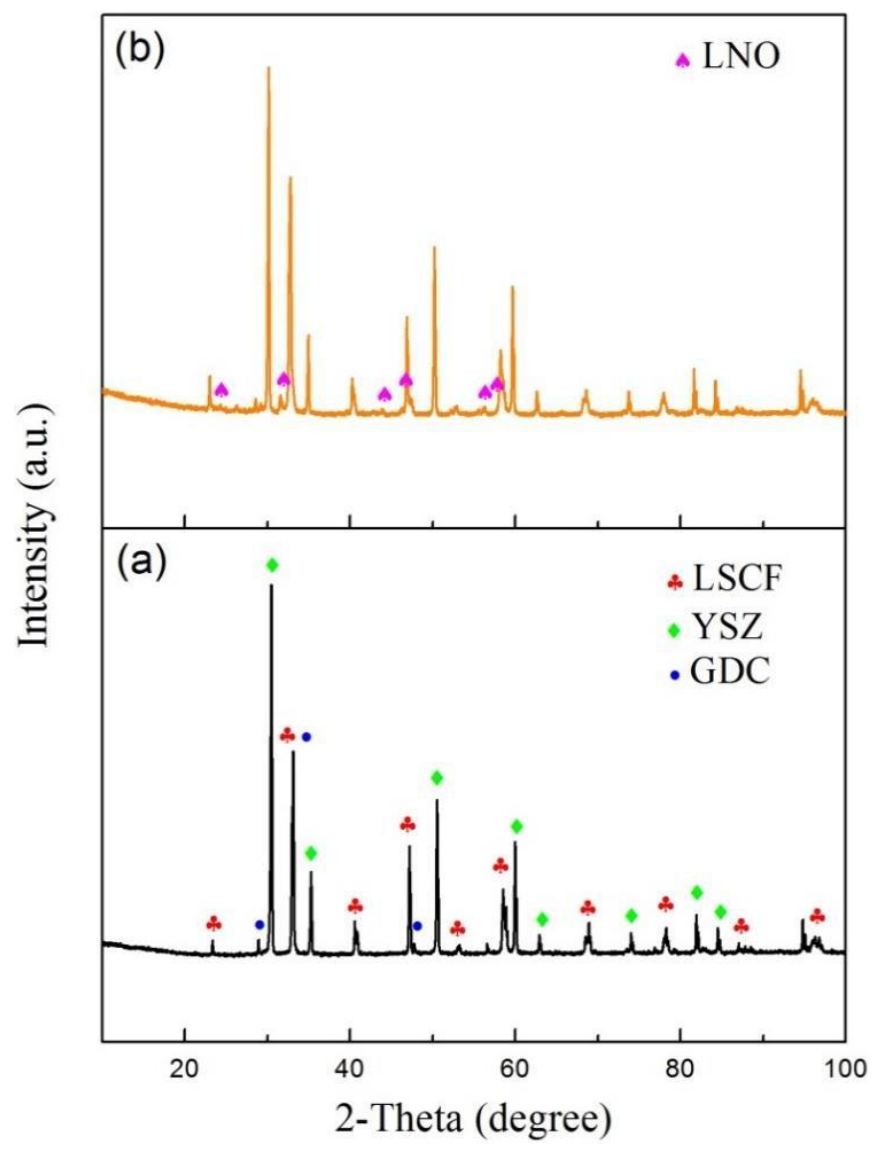

Figure 3.1 XRD patterns of (a) bare LSCF cathode and (b) LNOinfiltrated LSCF on GDC/YSZ electrolyte.

\subsubsection{Infiltration techniques}

To obtain uniform LNO nano-layer on LSCF surface, microstructure of LNO-infiltrated LSCF was studied as function of dispersant of the infiltration solution (TX100), drying rate, and concentration of infiltration solution, vacuum treatment and LNO loading.

Fig. 3.2 and 3.3 displays the microstructures of LNO-infiltrated LSCF with different heat treatment for low-temperature preprocesses and different mass of disperser. Reducing the heating rate from $5^{\circ} \mathrm{C} / \mathrm{min}$ to $2^{\circ} \mathrm{C} / \mathrm{min}$ for low-temperature preprocesses and introduction of dispersant TX100 with $0.9 \mathrm{~g}$ in $1 \mathrm{~mol}$ LNO solution improved the homogeneity of the solution and its evaporation, resulting in more uniform LNO nanoparticles. The change of concentration of infiltration solutions and the application of vacuum have little effect on the 
microstructure, as shown in Figure 3.4 and 3.5. Therefore, to simplify infiltration process, a $1 \mathrm{~mol} / \mathrm{L}$ infiltration solution was chosen in our future work without application of vacuum.

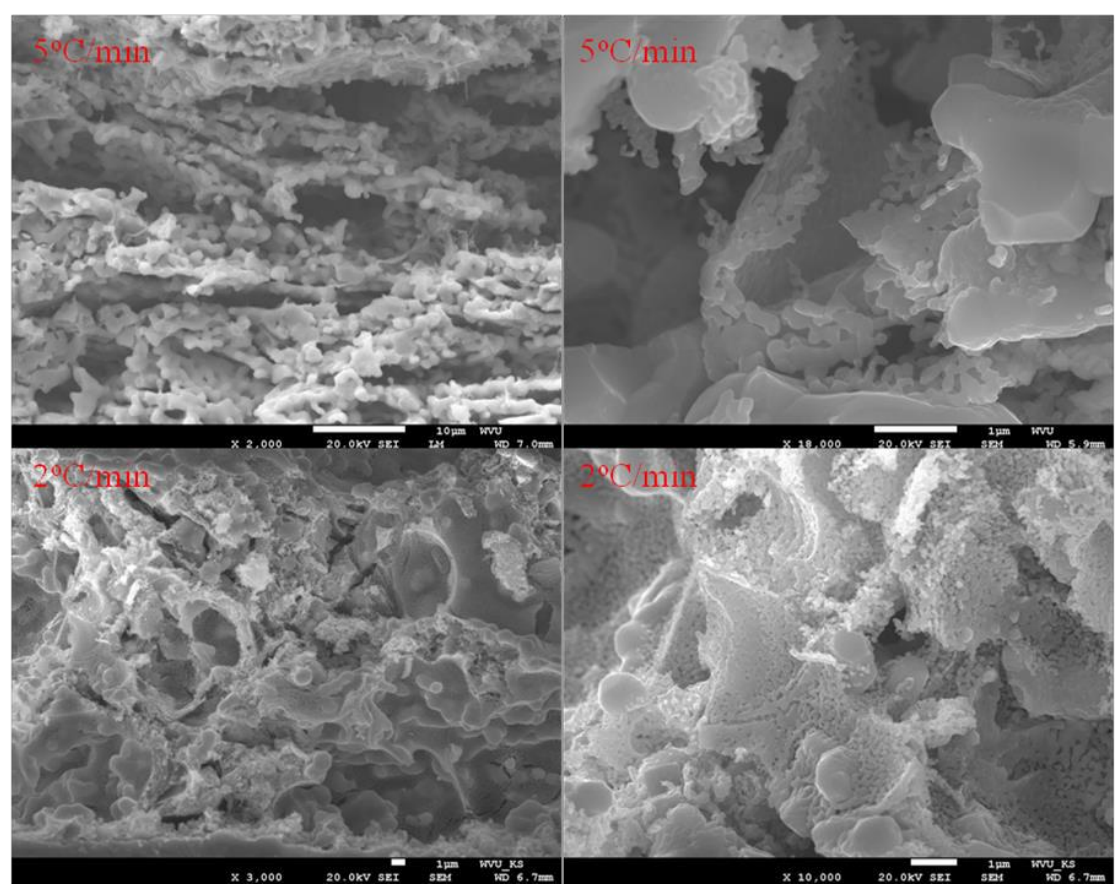

Figure 3.2 Microstructure of LNO-infiltrated LSCF electrodes with different heating rate for low-temperature preprocess

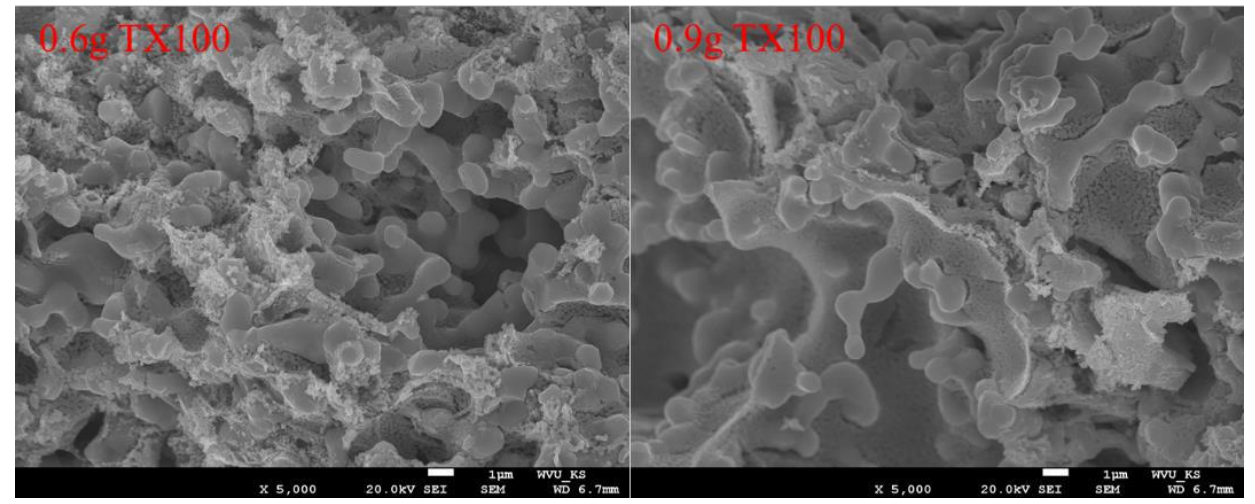

Figure 3.3 Microstructure of LNO-infiltrated LSCF electrodes with different mass of dispersant TX100 with $1 \mathrm{~mol} / \mathrm{L}$ LNO 


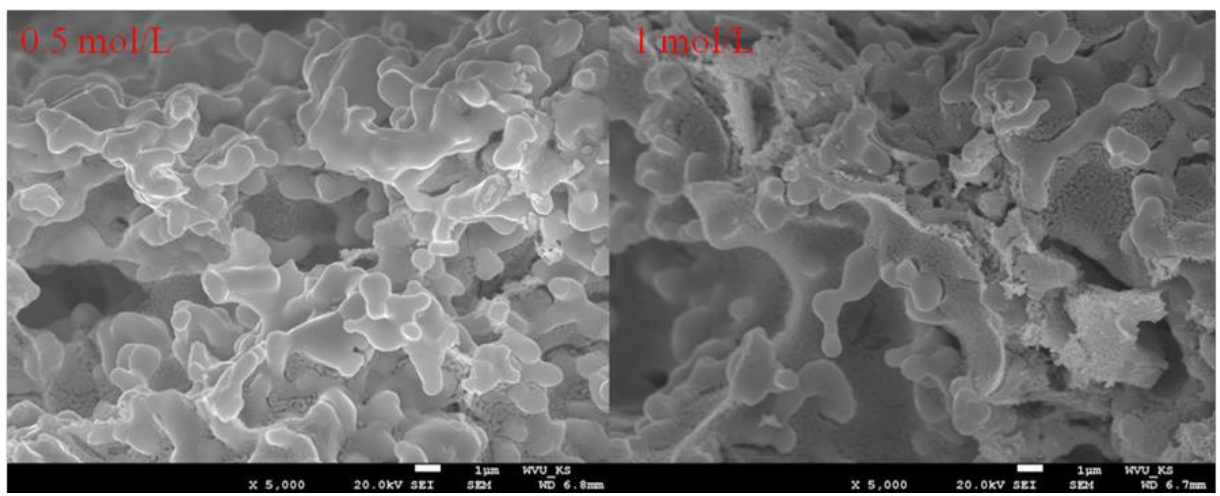

Figure 3.4 Microstructure of LNO-infiltrated LSCF electrodes with different concentration of LNO infiltration solutions

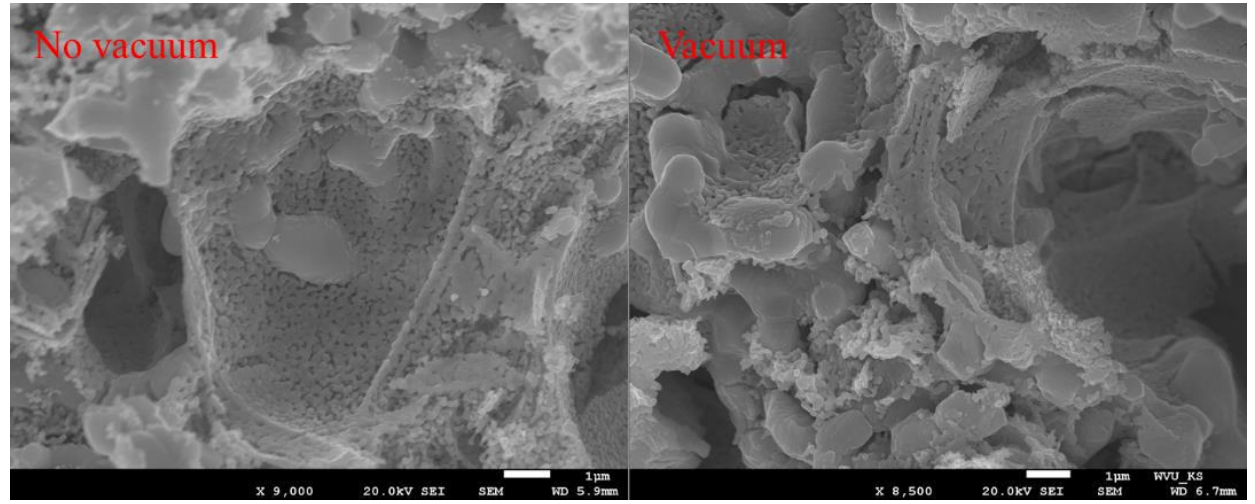

Figure 3.5 Microstructure of LNO-infiltrated LSCF electrodes with and without vacuum treatment during infiltration process

Fig. 3.6 displays the microstructures of LNO-infiltrated LSCF with different loading. The dispersant TX100 and ethanol were applied in the infiltration solution to enhance the surface tension and the dispersity of LNO nanoparticles. After repeating the infiltration process, LSCF cathode with different amounts of infiltrated LNO were obtained. With increasing the loading of LNO, the distribution of LNO nanoparticles in the LSCF substrate are islands, continuous films or multiple sheets. With 15.4 wt. \% LNO infiltration, a uniform LNO nano-layer almost completely covers the LSCF surface with particles of 50$150 \mathrm{~nm}$ diameter. By continuously increasing the LNO loading to about $20 \mathrm{wt}$. \%, a multiple layer of LNO formed and extra LNO nanoparticles were found on the LNO layer surface. Fig. 3.7 shows the typical electrochemical impedance spectra of LSCF/GDC/YSZ/GDC/LSCF symmetrical cells with different amount of LNO infiltration 
under OCV. A small quantity of LNO infiltration, less than $10 \mathrm{wt}$. \%, obtained the largest electrode resistance $0.16 \Omega \cdot \mathrm{cm}^{-2}$ because of the inadequate specific surface area and LNO/LSCF interface, but still lower than the blank LSCF one (about $0.3 \Omega \cdot \mathrm{cm}^{-2}$ ). A slight increase of cathode resistance was measured for the electrode with $20 \mathrm{wt}$ \% LNO loading, compared with the single layer LNO (15 wt. \%) infiltration electrode (0.06 VS. $0.08 \Omega \cdot \mathrm{cm}^{-}$ ${ }^{2}$ under $700^{\circ} \mathrm{C}$ ). Therefore, 15 wt. \% LNO loading, which covers the LSCF surface completely with a single uniform LNO nano-layer, was applied in the next work.
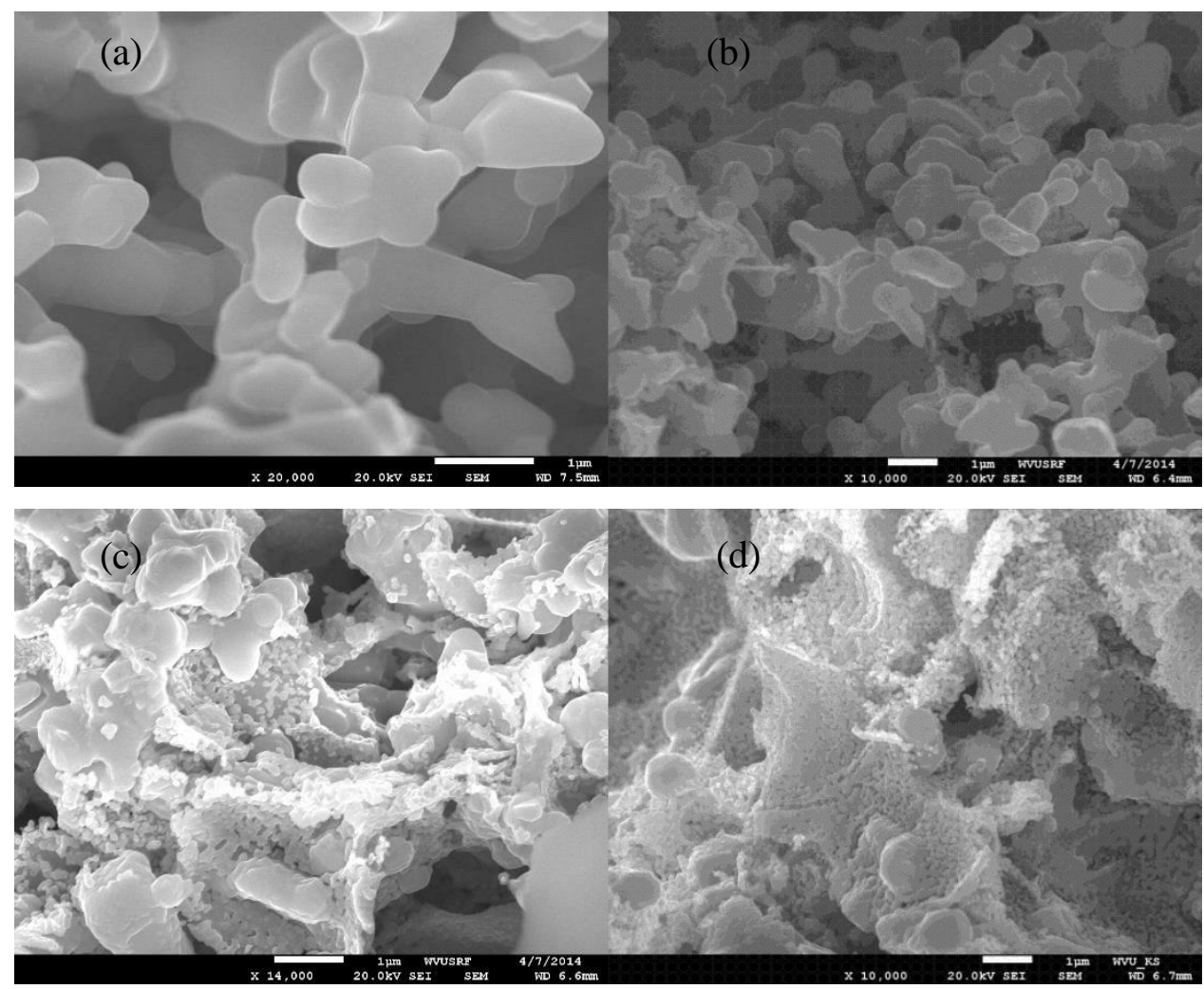

Figure 3.6 SEM image of LSCF cathode (a) without LNO infiltration or with (b) 8.60 wt. \%, (c) 15.4 wt. \% and (d) 20.3 wt. \% of LNO infiltration after $850^{\circ} \mathrm{C}$ sintering for $1 \mathrm{~h}$ 


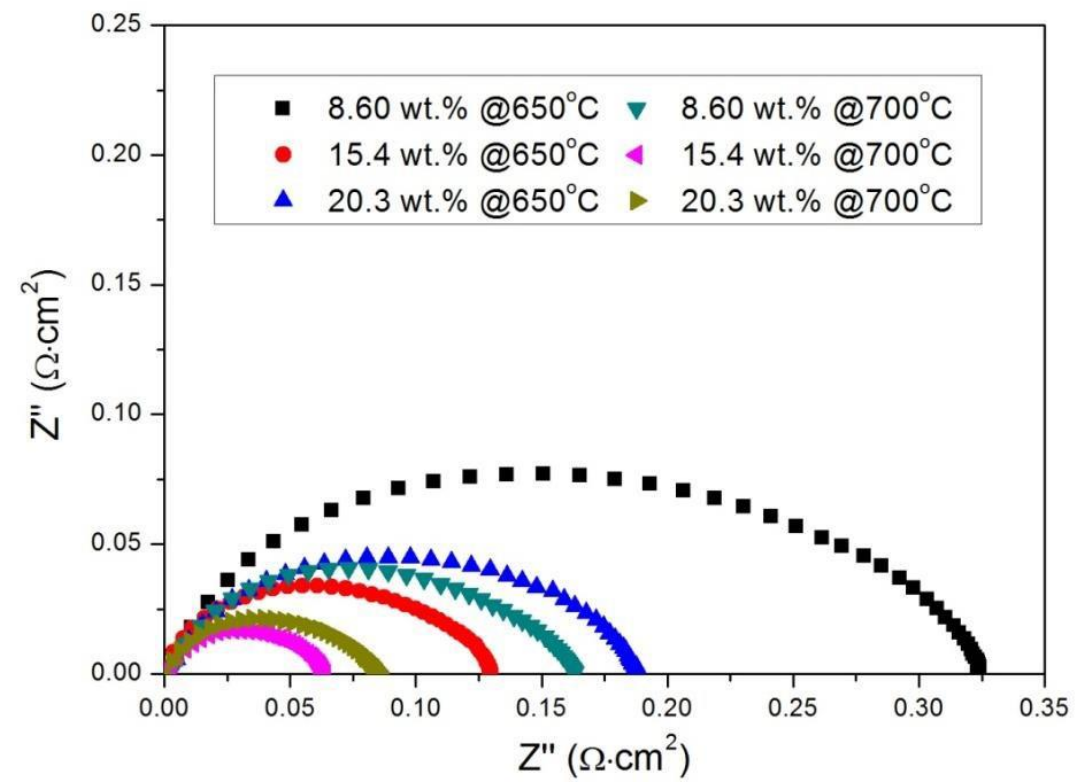

Figure 3.7 Typical electrochemical impedance spectra of symmetrical cells with different LNO loading under $\mathrm{OCV}$ at $650^{\circ} \mathrm{C}$ and $700^{\circ} \mathrm{C}$

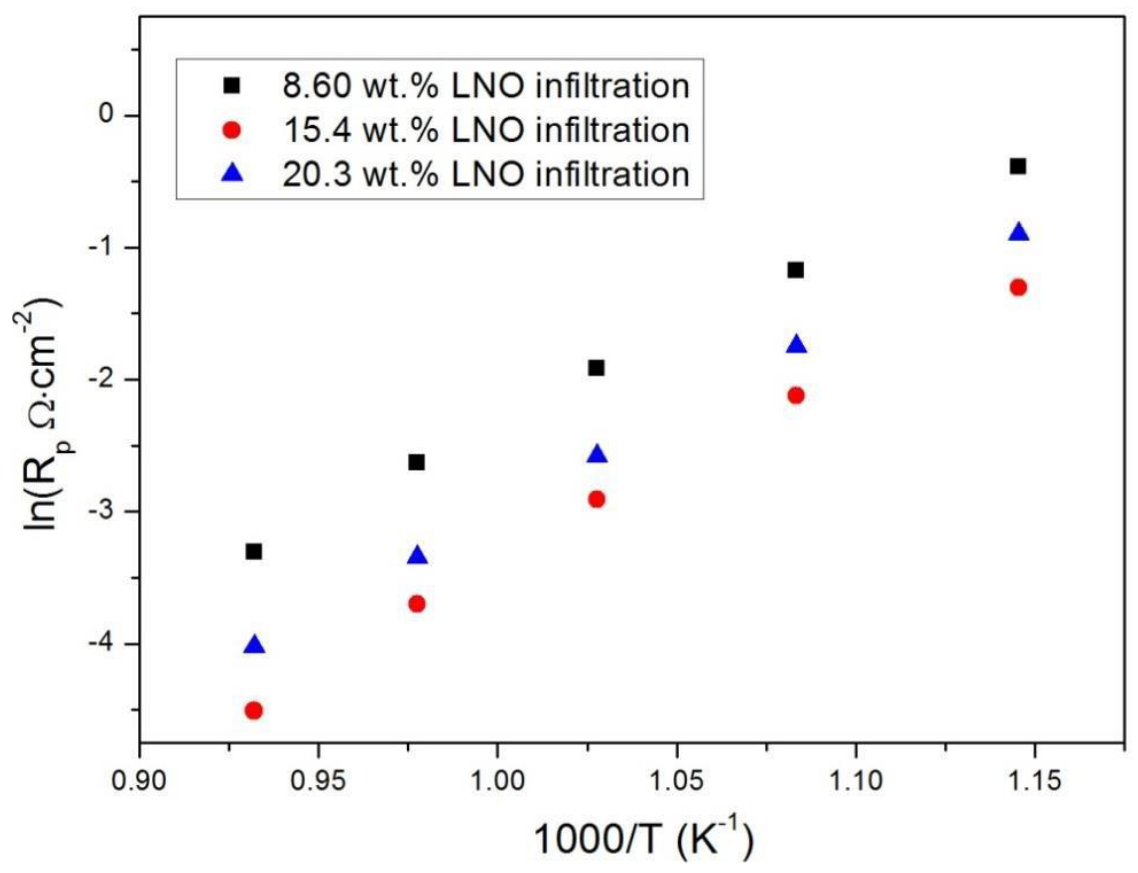

Figure 3.8 Cathode polarization resistant as function of measuring temperature for LSCF cathode with different LNO loading 3.3.3Electrochemical Performance of Infiltrated Cathode 
Representative EIS data for symmetric cells using bare LSCF or LNO-infiltrated LSCF cathodes collected at OCV in air at temperature of 700 and $750^{\circ} \mathrm{C}$, respectively, are shown in Fig. 3.9. Typical equivalent circuits have been used to deconvolute the measured data for the cathodes, as displayed in the insets of Fig. 3.9 (a) and (b), where $R$ is the ohmic resistance of the YSZ electrolyte, and $R_{n}$ and $\mathrm{Q}_{\mathrm{n}}$ indicate resistance and constant phase element of different electrode processes, respectively. In the case of LSCF cathode, two RQ elements of semi-circle arcs reflect the electrode polarization. An equivalent capacitance value $C_{1}\left(C_{n}=\left(R_{n} Q_{n}\right)^{1 / n} / R\right)$ in the range of $10^{-3}-10^{-2} \mathrm{~F} \cdot \mathrm{cm}^{-2}$ are obtained from high frequency response, which is typically attributed to charge transfer process according to literature $\left[{ }^{214,215}\right]$. For the $\mathrm{C}_{2}$ value of $\sim 10^{-2} \mathrm{~F} \cdot \mathrm{cm}^{-2}$, an intermediate behavior with a similar surface process (e.g. surface diffusion, adsorption/desorption, dissociation, etc.) between gas diffusion and charge-transfer is observed. For LNO-infiltrated LSCF cathode, an additional contribution at low frequency with a $\mathrm{C}_{3}$ value of $10^{0} \sim 10^{1} \mathrm{~F} \cdot \mathrm{cm}^{-2}$ has also been used for fitting, which may be assigned to gas diffusion as reported. Such an equivalent circuit model can well resolve the impedance plots of the symmetric cells using different cathodes. The actual polarization resistance of SOFC cathode $\left(R_{p}\right)$ can thereby be deduced from the electrode area and half sum of $R n$ values (symmetric cell).

The $R_{p}$ values for bare LSCF cathode are $1.34 \Omega \cdot \mathrm{cm}^{-2}$ at $700^{\circ} \mathrm{Cand} 0.62 \Omega \cdot \mathrm{cm}^{-2}$ at $750^{\circ} \mathrm{C}$, respectively, which are higher than those in literature reporting $R_{p} \approx 0.3-0.6 \Omega \cdot \mathrm{cm}^{-2}$ at $700^{\circ} \mathrm{Cand} 0.1-0.3 \Omega \cdot \mathrm{cm}^{-2}$ at $750^{\circ} \mathrm{C}$. This could be explained by the higher sintering temperature of $1150^{\circ} \mathrm{C}$ applied here for the LSCF cathode, leading to decreased surface area and more Sr segregation. For comparison, LNO-infiltrated LSCF cathode decreases the $R_{p}$ values to $0.042 \Omega \cdot \mathrm{cm}^{-2}$ at $700^{\circ} \mathrm{Cand} 0.023 \Omega \cdot \mathrm{cm}^{-2}$ at $750^{\circ} \mathrm{C}$. The reduction in cathode polarization resistance demonstrates that LNO infiltration enhances the electrochemical activity and surface exchange kinetics of LSCF electrodes. Temperature-dependent polarization resistance is characterized in Fig. 3.10, and activation energy (Ea) for the electrode polarization contribution can be calculated from the linear relationship of $\ln R_{p^{-}}$ $1 / \mathrm{T}$ by using the Arrhenius equation. To confirm the intrinsic influence of LNO, the electrode resistance of LSCF-infiltrated LSCF with similar loading was calculated and added in Fig. 3.10. It can be observed that the activation energy drops from $1.38 \mathrm{eV}$ to 1.06 
$\mathrm{eV}$ after the infiltration of LNO into LSCF. Hence, LNO is an effective catalyst to enhance ORR reaction kinetics and electro-catalytic activity of an LSCF cathode. The performance improvement could result from nanoscale LNO fine particles increasing surface active area, hetero-structured LSCF/LNO interface architecture offering more active reaction regions of $2 \mathrm{~PB}$ and $3 \mathrm{~PB}$, and/or favorable cation diffusion from LSCF to LNO facilitating oxygen exchange and transport. Comparing with LSCF self-infiltrated electrode, LNO-infiltrated LSCF cathode shows smaller resistance and activation energy. But it is far less than the reduction of LSC214 decorated LSC113 in resistant, which is consist with reported results of LSC214-decorated LSCF. The layered perovskite decoration has weaker effects on LSCF surface catalytic activity than that on LSC113. In consideration of narrow gap between LSCF-infiltrated LSCF and LNO-infiltrated LSCF electrode resistance in Fig. 3.10 , the largest driving force for the increased performance may relate to the microstructure change with LNO fine particles introducing.
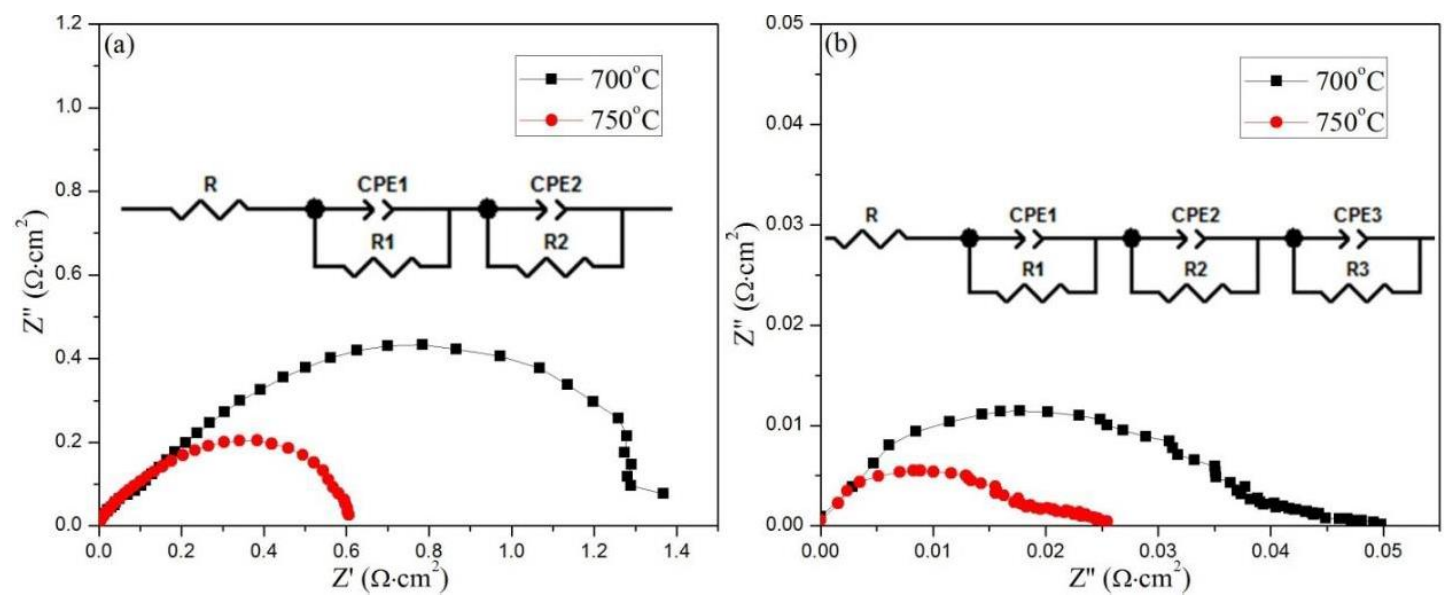

Figure 3.9 Typical electrochemical impedance spectra of symmetrical cells using LSCF cathodes (a) without and (b) with $\mathrm{LNO}$ infiltration under $\mathrm{OCV}$ at 700 and $750^{\circ} \mathrm{C}$ 


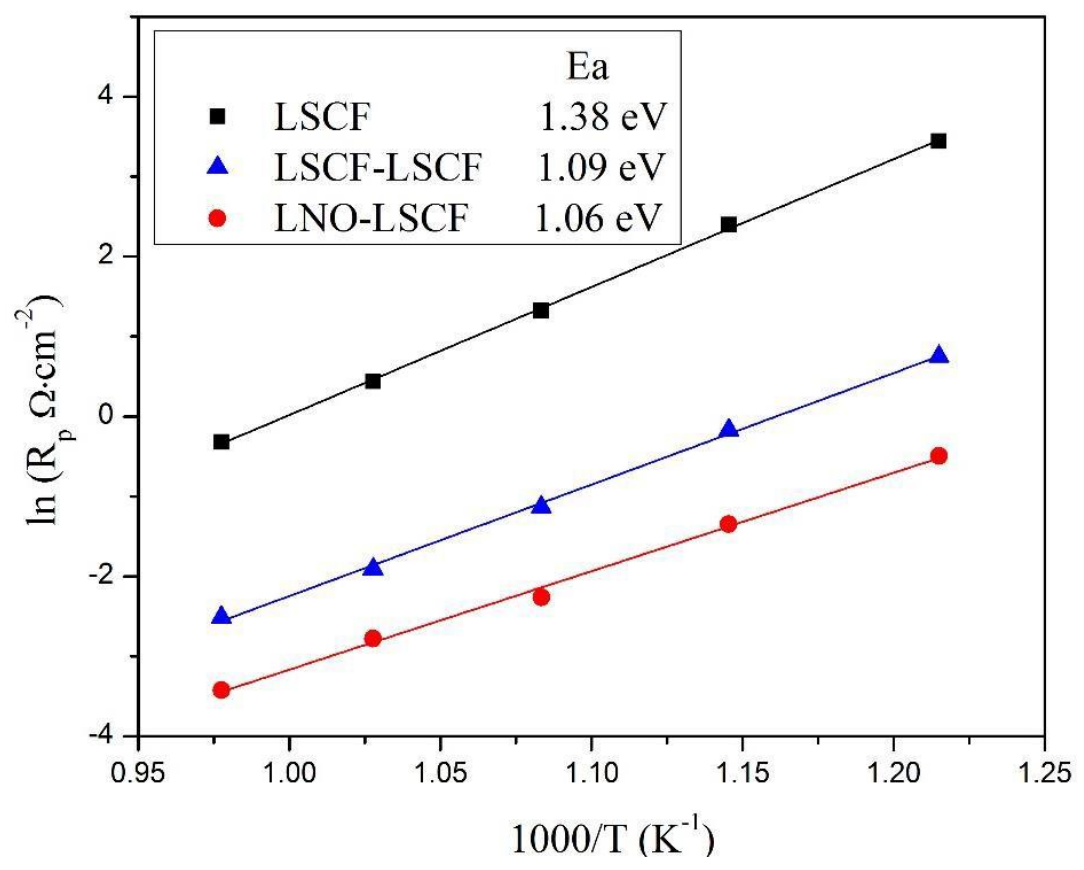

Figure 3.10 Typical electrochemical impedance spectra of symmetrical cells using LSCF cathodes with or without infiltration under OCV in air

Fig.3.11 presents the current-voltage characteristics and the corresponding power density for anode-supported fuel cells using bare LSCF or LNO-infiltrated LSCF cathodes during long-term operation of about $500 \mathrm{~h}$ at $750^{\circ} \mathrm{C}$. The open circuit voltages (OCVs) for both on-cells are very close to the theoretical value derived from the Nernst equation, which demonstrates that the cells are well-sealed. The power densities show an initial increase with time, indicating an activation process of cathode materials. The maximum power densities for the on cells with the cathodes of LSCF and LNO-infiltrated LSCF reach 418 and $697 \mathrm{~mW} \cdot \mathrm{cm}^{-2}$ after duration of around $100 \mathrm{~h}$, respectively. Hence, a $67 \%$ increase in the power density has been achieved through infiltration LNO into LSCF backbone. Even after $500 \mathrm{~h}$, LNO-infiltrated LSCF still delivers a power density of $637 \mathrm{~mW} \cdot \mathrm{cm}^{-2}$, higher than the pure LSCF electrode with power density of $392 \mathrm{~mW} \cdot \mathrm{cm}^{-2}$. 


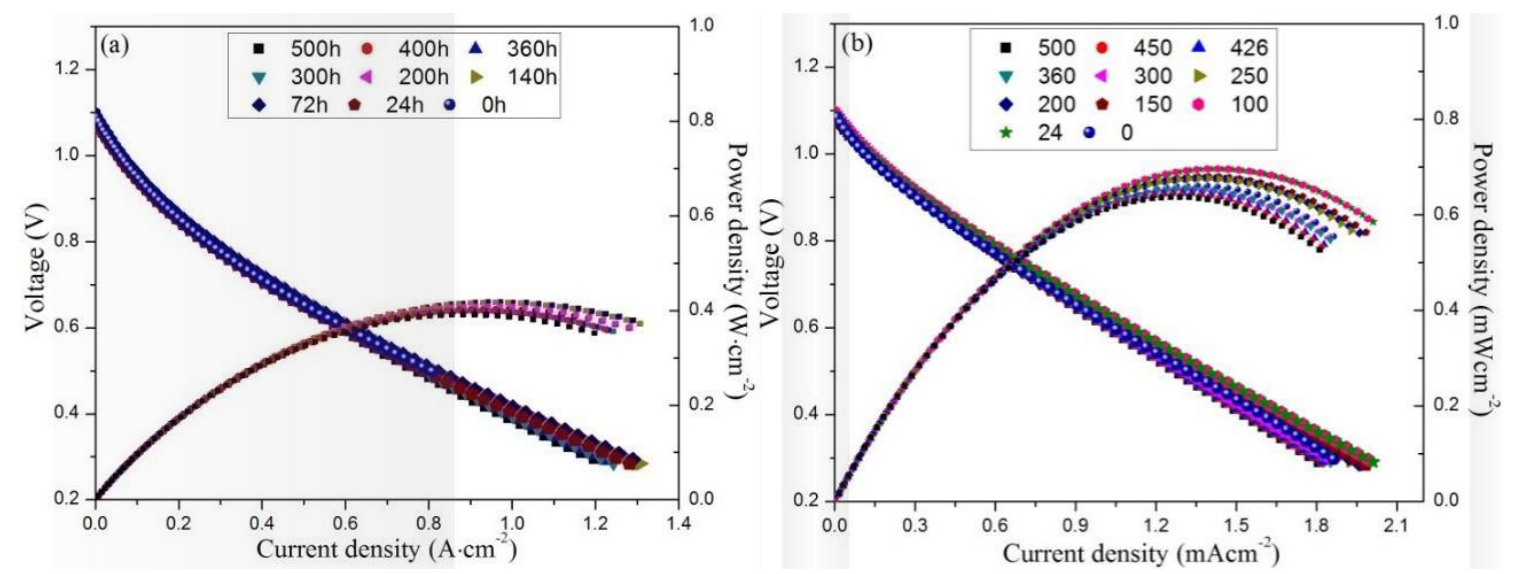

Figure 3.11 Cell voltage and power density as function of current density for (a) pure LSCF and (b) LNO-infiltrated LSCF measured at $750^{\circ} \mathrm{C}$ during cell operation of $500 \mathrm{~h}$

\subsubsection{Surface and Interface Oxygen Exchange Kinetics Behavior}

To further understand the enhancement of LNO-coated LSCF electrode, the surface and interface oxygen exchange kinetics behaviors were calculated using electronic conductivity relaxation (ECR). Conventional ECR technique can only be used for characterizing oxygen transport kinetics in single phase cathode. Based on improvement for the ECR data fitting method in our group's previous work $\left[{ }^{216}\right]$, this technique is further developed to characterize oxygen transport in the hetero-structured cathode.

Sandwich structure samples were fabricated for characterization of oxygen exchange behavior between the hetero-structured surface and bulk of LSCF cathode. After the system reaches equilibrium under the new oxygen partial pressure, the saturated concentration are $C_{\infty}^{\prime}$ and $C_{\infty}$ separately for the infiltrated material and LSCF. Oxygen flux was assumed as a constant and diffusion process inside the infiltrated layer was ignored due to its very small thickness. Oxygen transport can then be described with the three equations listed below.

$$
\begin{gathered}
C_{1}-C_{\infty}^{\prime}=\frac{J}{k_{\text {surface }}} \\
C_{2}-C_{\infty}=\frac{J}{k_{\text {interface }}}
\end{gathered}
$$




$$
C_{3}-C_{2}=\frac{J a}{D}
$$

Equation 3.1 is the surface exchange occurring on the gas/hetero-structured surface. Equation 3.2 represents the exchange occurring at the hetero-structured surface/LSCF bulk interface and Equation 3.3 showed the diffusion inside LSCF. Therefore, the total surface exchange coefficient can be deduced by combining Equation 3.2 and 3.3. The oxygen exchange coefficient at LNO/LSCF interface will be deduced based on a previous study for $\operatorname{LSCF}\left[{ }^{217}\right]$.

$$
\begin{gathered}
\frac{1}{k}=\frac{1}{k_{\text {interface }}}+\frac{1}{k_{\text {surface }}} \\
k_{\text {surace }}=\frac{c S_{V} k_{L N O}}{1-V}
\end{gathered}
$$

Where, $c$ is the thickness of LNO thin layer, $S_{V}$ is the specific surface area and V is the porosity

In the premier study, the oxygen transport process of SOFC cathodes including dense $\mathrm{La}_{2} \mathrm{NiO}_{4+\delta}(\mathrm{LNO})$ and $\mathrm{LNO} / \mathrm{LSCF} / \mathrm{LNO}$ has been studied to describe the change of oxygen chemical surface exchange coefficient $(k)$ and chemical diffusion coefficient $(D)$ as a function of oxygen partial pressure, based on the perturbation of oxygen stoichiometry during ECR measurements. However, the global minimum of the error for oxygen exchange coefficient is much larger. To obtain more accurate $\mathrm{k}$ value, the single process control sample is required, which reduces the diffusion equations to a single $k$ dependent equation as shown in Equation

$$
1-\sigma(\infty)-\sigma(0)-\sigma(0)=\exp \left(\begin{array}{r}
k t \\
-t
\end{array}\right)
$$

Where, $\sigma, k, t$ and $a$ represent conductivity, surface oxygen coefficient, time and sample thickness. Since the characteristic length for LNO and LSCF is 50-500 $\mu \mathrm{m}$, the thickness of the $\mathrm{k}$ single process control sample should less than the characteristic length. In our experiment, the dense LNO and LNO/LSCF thin film based on GDC substrate were prepared by air brush spraying a thickness of about $50 \mu \mathrm{m}$. Fig. 3.12 shows a typical set of normalized conductivity relaxation data and the correspondingly fitted curves of 
$\mathrm{LSCF} / \mathrm{GDC}$ and $\mathrm{LNO} / \mathrm{LSCF} / \mathrm{GDC}$ samples at $700^{\circ} \mathrm{C}$, respectively, for reduction steps 
switching $\mathrm{PO}_{2}$ from 0.20 atm to 0.18 atm as function of time. The fitted oxygen surface exchange $k$ of LSCF and LNO/LSCF at different $\mathrm{PO}_{2}$ are listed in Table 3.1.From Fig. 3.12, the total relaxation time for $\mathrm{LSCF}$ is about $120-160 \mathrm{~s}$ when lowering $\mathrm{PO}_{2}$ from 0.21 to 0.18 atm, which is longer than LNO/LSCF sample about 40-60 s. The total surface oxygen exchange coefficient of LSCF and LNO/LSCF is about $9.02 \times 10^{-5} \mathrm{~cm} / \mathrm{s}$ and $1.9 \times 10^{-4} \mathrm{~cm} / \mathrm{s}$

at $700^{\circ} \mathrm{C}$ under air condition, respectively, which means LNO layer on LSCF substrate involved with elements inter-diffusion, would enhance the surface ORR rate. Elemental inter-diffusion in LNO layer may enhance the surface oxygen exchange rate compared with the pure LNO one. Meanwhile, surface oxygen exchange coefficient of the LSCF and total LNO/LSCF decreases as a function of the square root of oxygen particle pressure. According to the thermodynamic properties and oxygen exchange kinetics, the dependence of surface oxygen exchange coefficient on temperature and oxygen particle pressure reflects the oxygen reduction mechanism. The $k$ order in $\mathrm{PO}_{2}$ is about $1 / 2$ in the experiment, which usually means that the rate limiting step in ORR is oxygen incorporation into surface oxygen defect site $\left[{ }^{218}\right]$. According to the $k_{\mathrm{La} 2 \mathrm{NiO} 4+\delta}$ and $k_{\mathrm{LNO}-\mathrm{LSCF} \text { total }}$ it can be deduced from Equation 3.5 that the interface oxygen exchange coefficient between LNO and LSCF is about $3 \times 10^{-4} \mathrm{~cm} / \mathrm{s}$. The higher $k_{\text {interface }}$ and $k_{\text {surface }}$ with respect to $k_{\mathrm{LSCF}}\left(9.02 \times 10^{-5} \mathrm{~cm} / \mathrm{s}\right)$, indicates that the incorporation rate of oxygen into LSCF is enhanced when using LNO/LSCF cathode. The enhancement of electrochemical properties for LNO-infiltrated LSCF cathode not only results from the high surface area of nanoparticles, but also from the rapid intrinsic oxygen incorporation rate on the surface and LNO/LSCF interface.
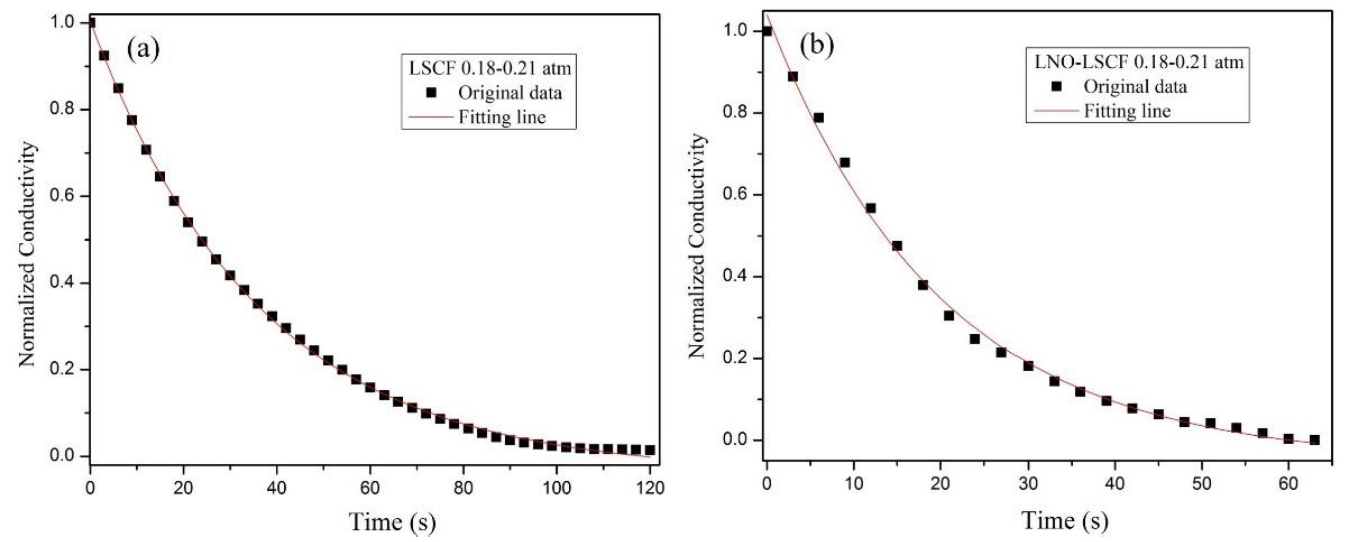
Figure 3.12 Typical ECR data and fitting results of (a) LSCF and (b) LNO-coated LSCF (LNO/LSCF) samples at $700^{\circ} \mathrm{C}$ under air conditions
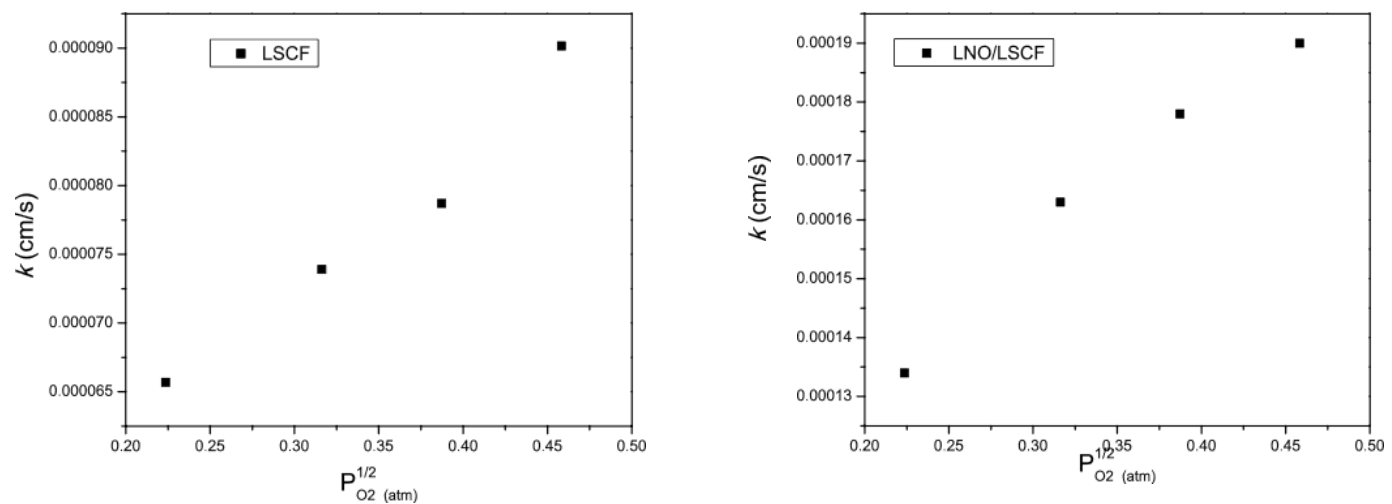

Figure 3.13 Surface oxygen exchange coefficient of (a) LSCF and (b) LNO-coated LSCF (LNO/LSCF) samples as a function of $\mathrm{PO}_{2}{ }^{1 / 2}$ at $700^{\circ} \mathrm{C}$

Table 3.1 The surface oxygen exchange coefficient $(k)$ and of $\mathrm{La}_{2} \mathrm{NiO}_{4+\delta}$, LSCF and LNOcoated LSCF (LNO/LSCF) cathode at $700^{\circ} \mathrm{C}$ under different oxygen pressure.

\begin{tabular}{|c|c|c|c|c|}
\hline $\mathrm{PO}_{2}(\mathrm{~atm})$ & 0.21 & 0.15 & 0.10 & 0.05 \\
\hline$k_{\mathrm{La} 2 \mathrm{NiO} 4+\delta \text { surface }}(\mathrm{cm} / \mathrm{s})$ & 3.95 E-04 & $3.54 \mathrm{E}-04$ & $3.26 \mathrm{E}-04$ & $2.83 \mathrm{E}-04$ \\
\hline$k_{\mathrm{LSCF}}(\mathrm{cm} / \mathrm{s})$ & $\begin{array}{l}9.02 \mathrm{E}-05 \\
\pm 7.94 \mathrm{E}-07\end{array}$ & $\begin{array}{l}7.87 \mathrm{E}-05 \\
\pm 5.83 \mathrm{E}-07\end{array}$ & $\begin{array}{l}7.37 \text { E-05 } \\
\pm 6.32 \text { E-07 }\end{array}$ & $\begin{array}{l}6.57 \mathrm{E}-05 \\
\pm 7.26 \mathrm{E}-07\end{array}$ \\
\hline$k_{\text {LNO-LSCF }}$ total $(\mathrm{cm} / \mathrm{s})$ & $\begin{array}{l}1.90 \mathrm{E}-04 \\
\pm 2.08 \mathrm{E}-06\end{array}$ & $\begin{array}{l}1.78 \mathrm{E}-04 \\
\pm 3.95 \mathrm{E}-06\end{array}$ & $\begin{array}{l}1.63 \mathrm{E}-04 \\
\pm 1.34 \mathrm{E}-06\end{array}$ & $\begin{array}{l}1.34 \mathrm{E}-04 \\
\pm 4.32 \mathrm{E}-06\end{array}$ \\
\hline$k_{\text {LNO-LSCFinterface }}(\mathrm{cm} / \mathrm{s})$ & 3.66 E-04 & $3.58 \mathrm{E}-04$ & $3.26 \mathrm{E}-04$ & $2.55 \mathrm{E}-04$ \\
\hline
\end{tabular}

\subsubsection{Surface and Bulk Elements Distribution and Crystal Structure of LNO Layers}

EDAX results of LNO-infiltrated LSCF cathode indicate that the diffusion of $\mathrm{Co}, \mathrm{Sr}$ and $\mathrm{Fe}$ occurs from LSCF to LNO phase. The extraction of the cations from LSCF perovskite lattice reveals the intrinsic instability of the LSCF cathode. As an acceptor, LNO materials could positively take advantage from the cation diffusion to enhance surface catalytic properties and ORR kinetics. The cobaltic electrode usually feature fast oxygen surface exchange and high electro-catalytic activity for oxygen reduction. It has been reported that 
doped-Fe LNO, at least for small amounts of Fe loading have no influence on the electrocatalytic properties of LNO materials. As to Sr doping into LNO, a possible reaction according to charge compensation may appear to increase the concentration of electron hole, as below,

$$
\underset{\mathrm{SrO}}{\mathrm{Si}}+\mathrm{Ni}_{\mathrm{Ni}}^{\times} \longleftrightarrow 2 \mathrm{Sr}_{\mathrm{La}}^{\prime}+\mathrm{Ni} i_{N i}^{\bullet}+\mathrm{O}_{\mathrm{O}}^{\times}
$$

These facts might lead to the improved electrochemical performance and fast surface exchange of the LSCF based cathode. Certainly, evidence to support these hypotheses should be provided by evaluating the valence and amount of the $\mathrm{Fe}(\mathrm{IV}) / \mathrm{Fe}(\mathrm{III}), \mathrm{Co}(\mathrm{IV}) /$ Co (III), Ni(IV)/ Ni (III) and Sr ( II ) in the LNO infiltrant during operation. Further work on detailed characterizations associated with ORR process and kinetics of the composite cathodes are shown below.

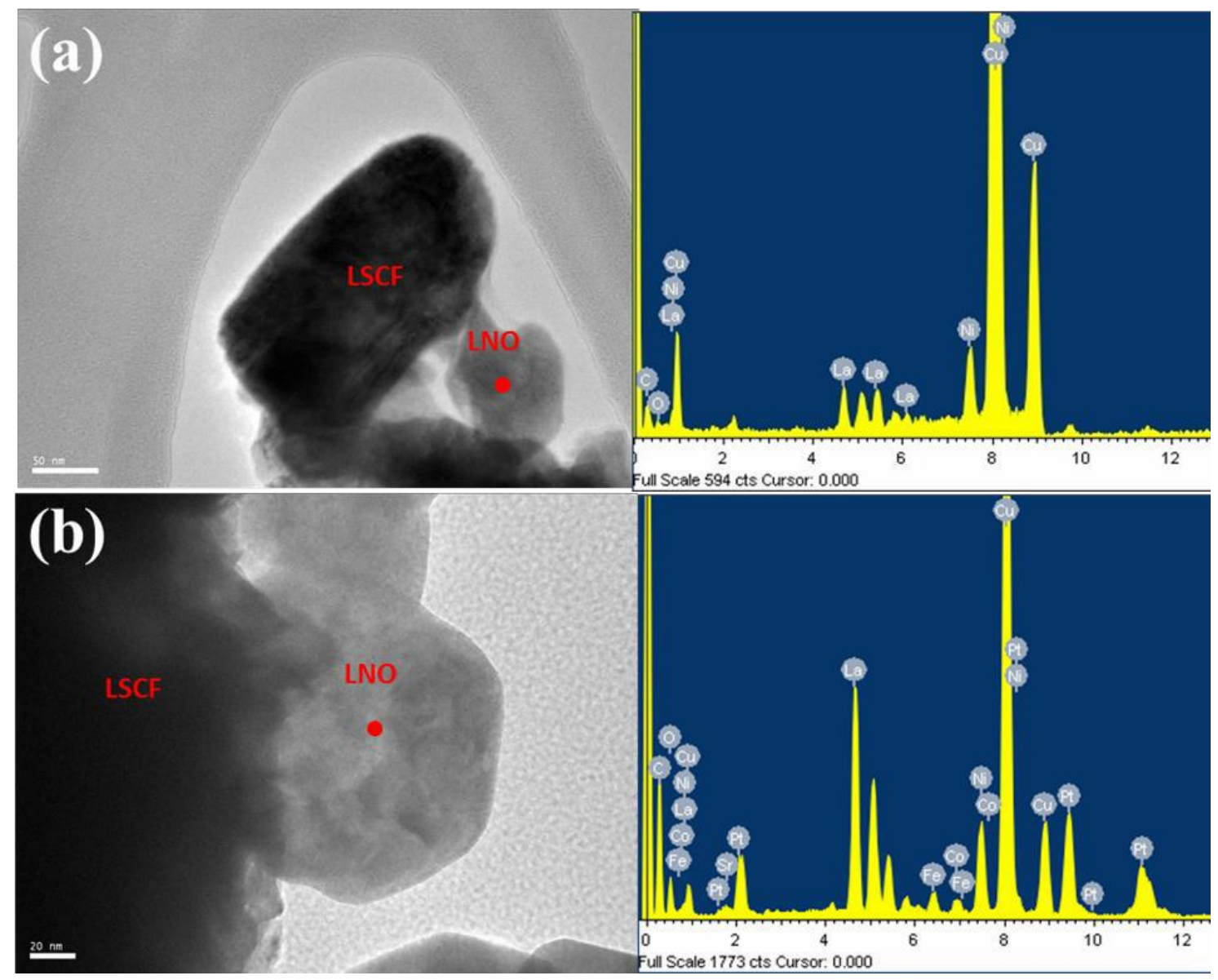


Figure 3.14 TEM images and EDAX spectra of LNO-infiltrated LSCF cathode (a) before on-cell testing and (b) after long term testing

To acquire useful information about LNO/LSCF interface and surface, dense LNO-coated LSCF samples have been prepared by spin-coating and air-brushed dry pressing. The XRD pattern of the LNO-coated LSCF materials shows that no extra lines than pure RuddlesdenPopper (RP) phase (PDF\# 34-0314) are observed in the XRD diagram. Figure 3.15 displays the SEM cross-section of the LNO-coated LSCF sample. It can be observed that a dense LNO layer of about $4.0 \mu \mathrm{m}$ thickness was coated on the LSCF backbone. In the meantime, strong bonding between LNO and LSCF should be expected from the high temperature cofiring. Sr and Co diffusion from LSCF to LNO occurs along with EDX line scans can be detected from Fig. 3.16, which is consistent with the previous TEM/EDX data in infiltrated LSCF cathode. XPS measurements reveal the changes in the surface composition of $\mathrm{La}_{2} \mathrm{NiO}_{4+\delta}$ based layer. Fig. 3.17 shows the XPS spectra of different elements on the coated $\mathrm{La}_{2} \mathrm{NiO}_{4+\delta}$ surface after $1350^{\circ} \mathrm{C}$ sintering. The obvious $\mathrm{Ni} 3 \mathrm{p}$ and $\mathrm{Co} 2 \mathrm{p}$ peaks were detected in accordance with EDX results. The influence of Co diffusion on crystal structure and oxygen defect concentration was investigated by XRD refinement and iodometric titration method discussed later. The weak $\mathrm{Sr} 3 \mathrm{~d}$ peak was found on the coated $\mathrm{La}_{2} \mathrm{NiO}_{4+\delta}$ surface. According to reported reference, two sets of $\mathrm{Sr} 3 \mathrm{~d}$ doublets need to be used in XPS fitting, as shown in Fig. 3.18. The peak with high bonding energy is correspond to the position of $\mathrm{Sr}$ in $\mathrm{SrO} / \mathrm{Sr}(\mathrm{OH})_{2}$, and the peak with low bonding energy can be attributed to $\mathrm{Sr}$ in the octahedron structure. The relative intensity of $\mathrm{SrO} / \mathrm{Sr}(\mathrm{OH})_{2}$ increase for LSCF after $-0.2 \mathrm{~V}$ polarization, while the similar weak $\mathrm{Sr} 3 \mathrm{~d}$ peaks were detected in LNO-LSCF sample after polarization treatment, which indicates suppression effect of LNO addition, and the concentration of $\mathrm{SrO} / \mathrm{Sr}(\mathrm{OH})_{2}$ on coated LNO surface seems to change little after similar polarization treatment. It means that $\mathrm{La}_{2} \mathrm{NiO}_{4+\delta}$ would be an acceptor for $\mathrm{Sr}$ segregation or diffusing from LSCF substrate to relieve the stability problem of LSCF. 


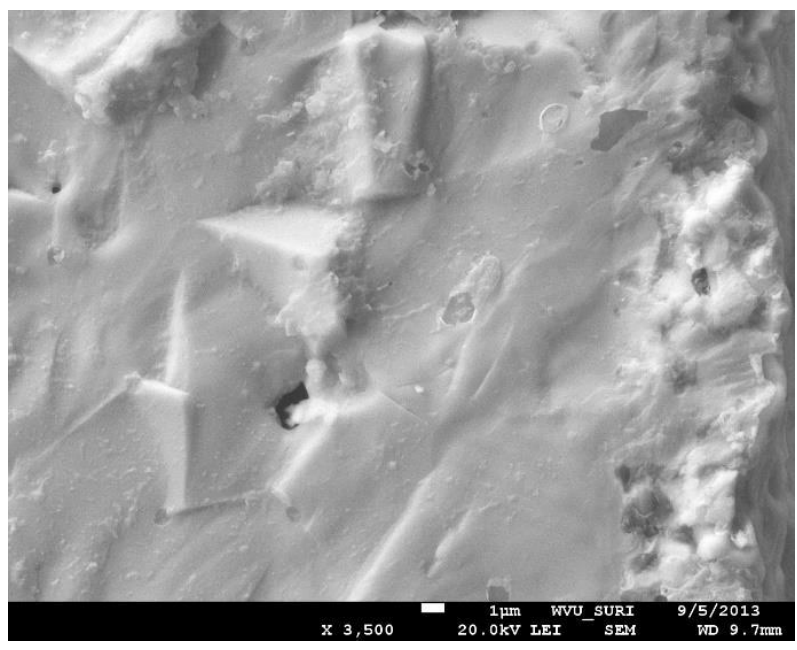

Figure 3.15 Cross-section morphology of LNO-coated LSCF material
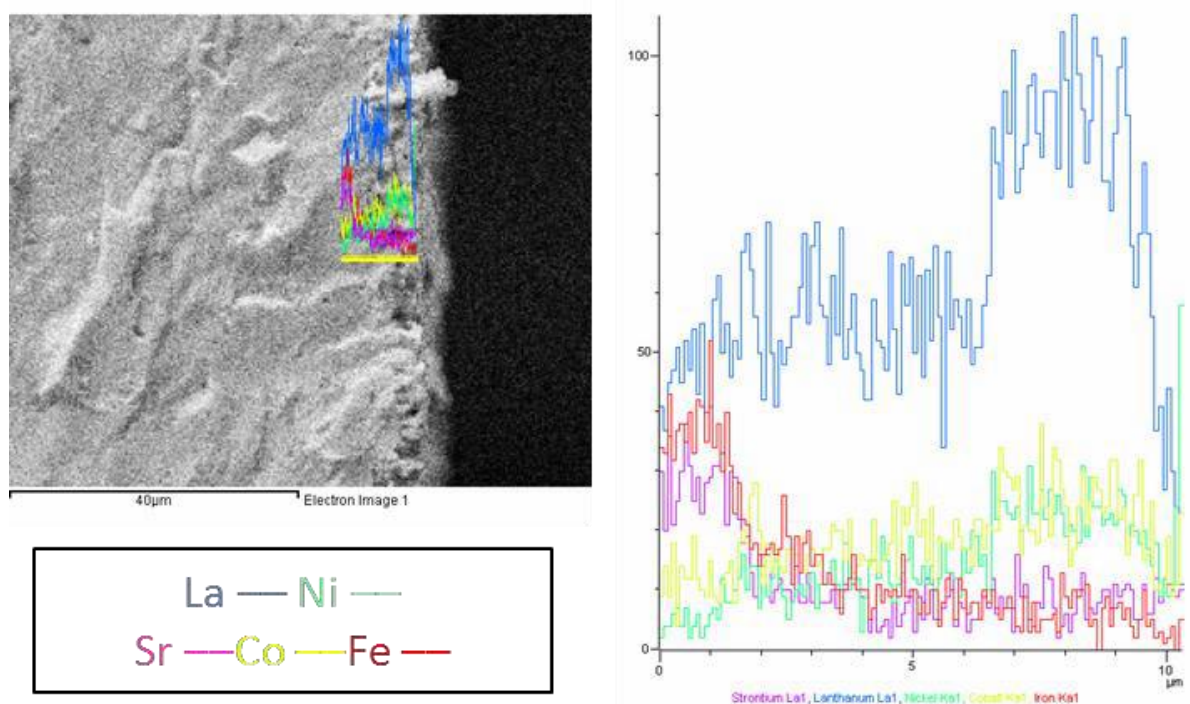

Figure 3.16 EDX line scan results for LNO-coated LSCF 

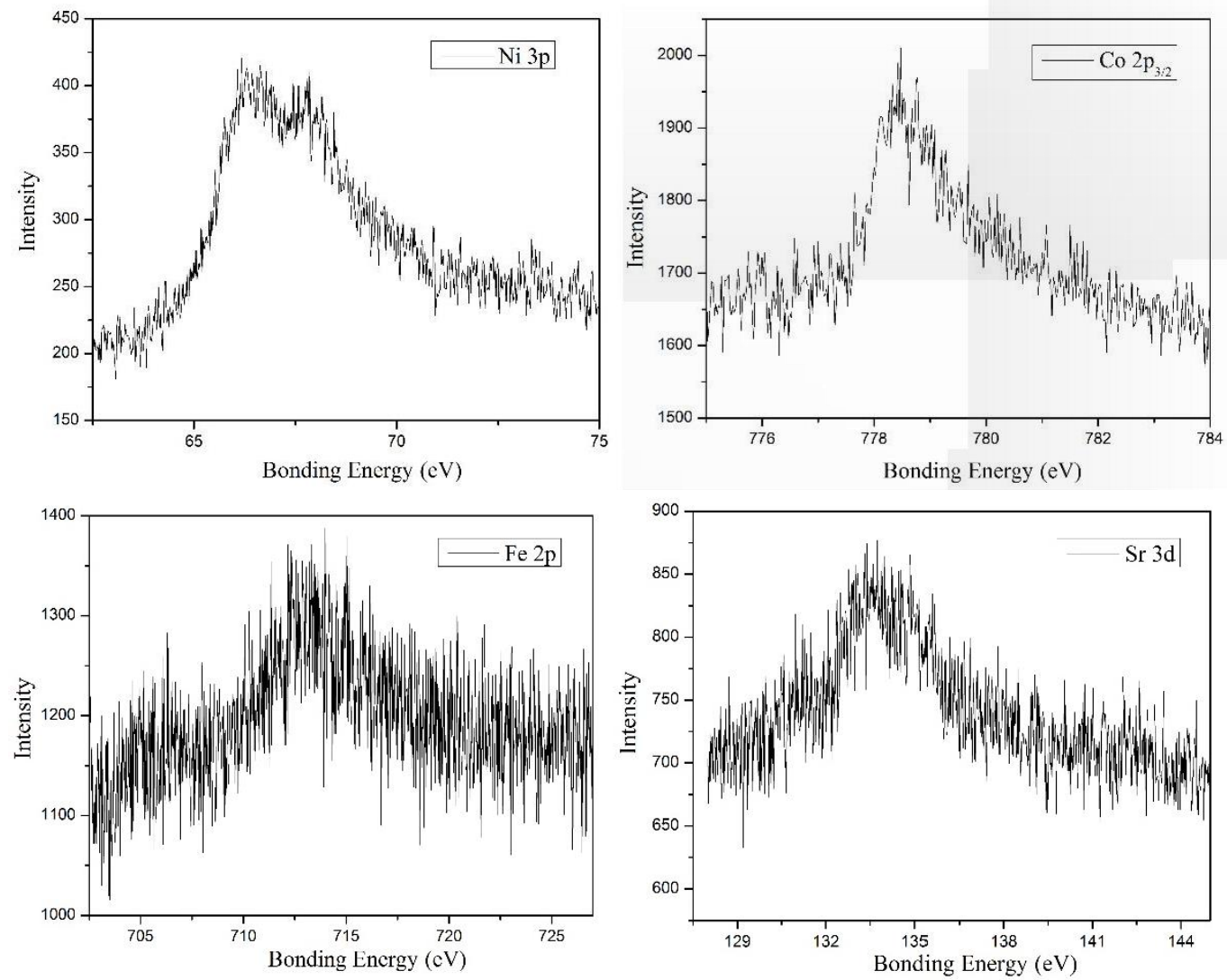

Figure $3.17 \mathrm{Ni} 3 \mathrm{p}$, Co 2p, Fe 2p and Sr 3d XPS spectra of $\mathrm{La}_{2} \mathrm{NiO} 4-\delta$-coated LSCF surface 

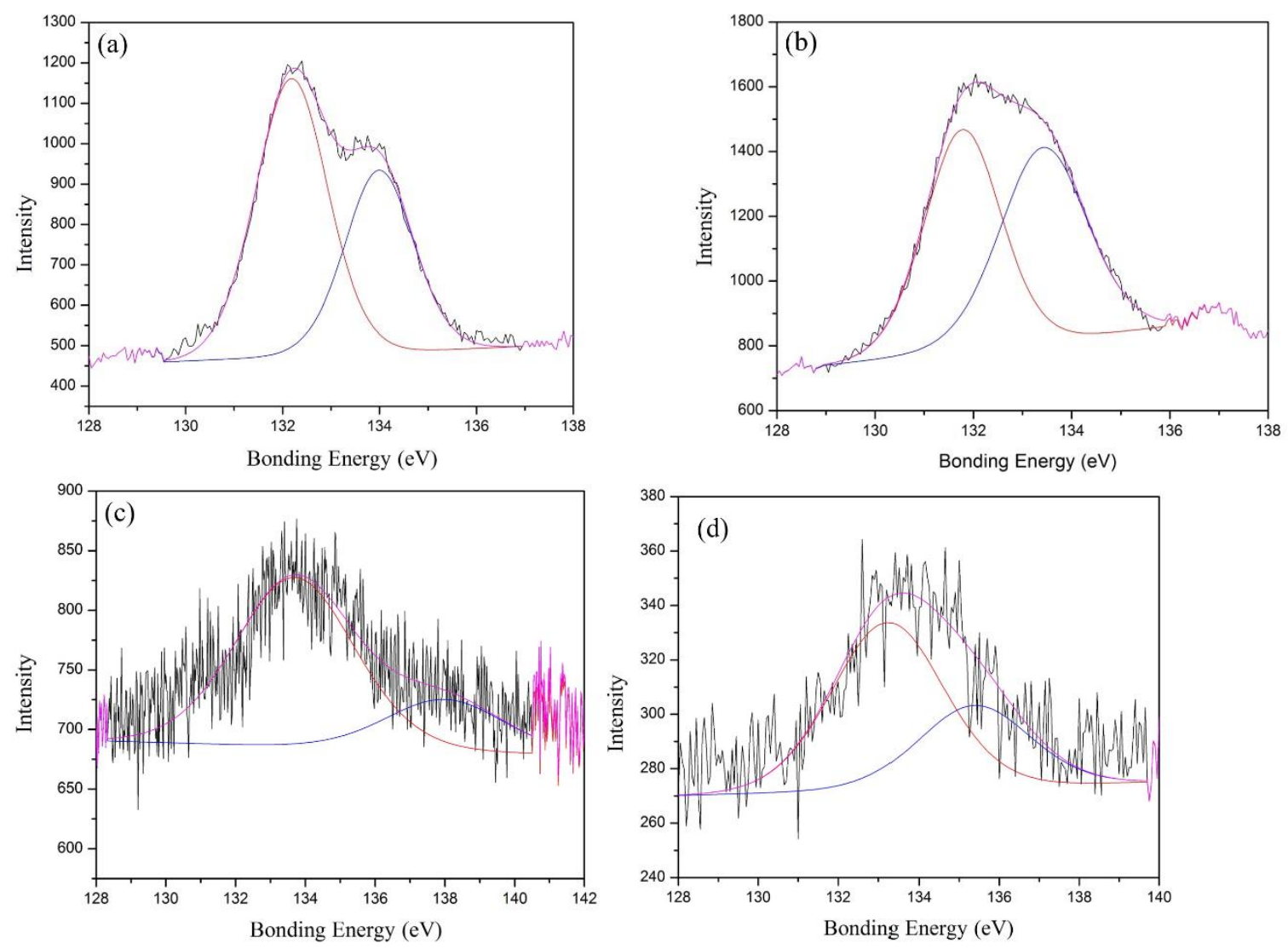

Figure 3.18 Sr 3d XPS spectra of LSCF (a) fresh surface; (b) surface after polarization and of $\mathrm{La}_{2} \mathrm{NiO}_{4+\delta}$-coated LSCF (c) fresh surface and (d) surface after polarization

In addition, XRD refinements using GASA system have been carried out for dense LNOLSCF to obtain the detailed crystal structure information and explain the $k$ and $D$ variation, such as cell parameters and Ni-O/La-La bond distance. A shifted Cherbyshev background function and pseudo Voigt peak shape were used in the refinement process. A best fit to the experimental data was obtained in the tetragonal $\mathrm{I} / \mathrm{mmm}$ space group with the La occupying $(0,0, \mathrm{z})$ position, $\mathrm{Ni}$ occupying $(0,0,0)$ position and two kinds of oxygen occupying $(0,0, \mathrm{z})$ and $(0,1 / 2,0)$ position. Refined crystal structure information was shown in Fig. 3.27 and Table 3.2-3.5. For the bond length analysis, we can find that the La-O2 $(* 1)$ bond length decreases after LNO applied on LSCF surface. Fig. 3.28 shows the crystal structure of $\mathrm{LNO}$, the distance of $\mathrm{La}-\mathrm{O} 2(* 1)$ bond represent the length of AO rock salt layer which is critical for the oxygen interstitial transport located in the oxygen or lanthanum tetrahedron. The decrease of $\mathrm{La}-\mathrm{O} 2(* 1)$ will reduce the space of interstitial 
oxygen transport channels and further influence the oxygen transport properties. The oxygen atomics occupy in two positions shown in Table. 3.3 and 3.4. It indicates that the concentration of oxygen vacancy decreases in LNO layer coated on LSCF surface. If the interstitial oxygen atomics were added in the crystal model occupying the $16 \mathrm{n}$ site in tetragonal, after modification, the occupancy of interstitial is higher in LNO-LSCF than that in pure LNO phase. Most researchers believed that the oxygen incorporation processes in LNO are controlled by interstitial oxygen state in AO rock salt layer. The higher interstitial oxygen and oxygen vacancy concentration can enhance surface oxygen incorporation and transport rate on LSCF/LNO surface and interface. The oxygen defect concentrations were determined with iodometric titration based on the oxidation of iodide into iodine. In iodometric titration, the oxidizing agent (transition metal) reacts with the excessive $\mathrm{KI}$ in neutral or weak acidic medium firstly based on equation 3.8 , and then the valence of oxidizing agent is identified by titration of oxidized iodine with thiosulfate according to equation 3.9. Oxygen defect concentrations are therefore calculated according to charge compensation effects. Starch is used as indicator. At the end point, the blue or violet color of starch indicator disappears due to change of iodine to iodide. The total chemical reactions are listed below:

$$
\begin{gathered}
\mathrm{I}^{-} \leftrightarrow \mathrm{I}_{2}+2 e \\
2 \mathrm{Na}_{2} \mathrm{~S}_{2} \mathrm{O}_{3}+\mathrm{I}_{2} \rightarrow \mathrm{Na}_{2} \mathrm{~S}_{4} \mathrm{O}_{6}+2 \mathrm{NaI}
\end{gathered}
$$

The reducing $\mathrm{Na}_{2} \mathrm{~S}_{2} \mathrm{O}_{3}$ solution needs to be standardized at the beginning in accordance with equation 3.8 and 3.9 by using a fixed amount of strong oxidizer $\left(\mathrm{KMnO}_{4}, \mathrm{~K}_{2} \mathrm{Cr}_{2} \mathrm{O}_{7}\right)$ as the oxidizing agent.

$$
6 \mathrm{KI}+\mathrm{K}_{2} \mathrm{Cr}_{2} \mathrm{O}_{7}+7 \mathrm{H}_{2} \mathrm{SO}_{4} \rightarrow 3 \mathrm{I}_{2}+\mathrm{Cr}_{2}\left(\mathrm{SO}_{4}\right)_{3}+4 \mathrm{~K}_{2} \mathrm{SO}_{4}+7 \mathrm{H}_{2} \mathrm{O}
$$

The results of iodometric titration, as shown in Table 3.6, also indicated that the adsorption of $\mathrm{Co}$ and $\mathrm{Sr}$ in LNO increases the concentration of oxygen defects, and then influences the oxygen exchange and transport properties of LNO. 

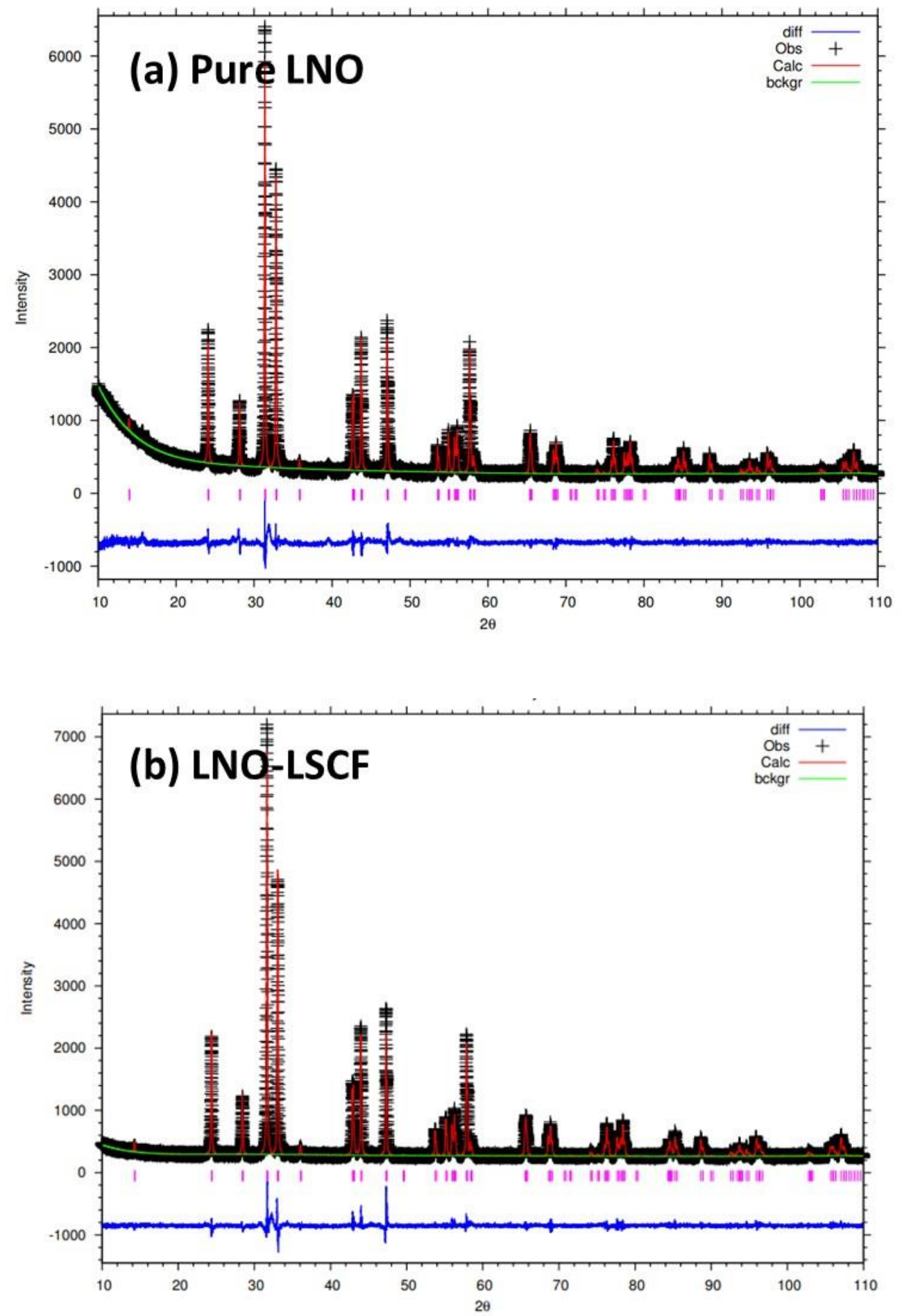

Figure 3.19 XRD data for (a) pure LNO pellet and (b) LNO layer coated on LSCF surface recorded at room temperature. (+) represent the experimental data points, solid red line is refined data fit 


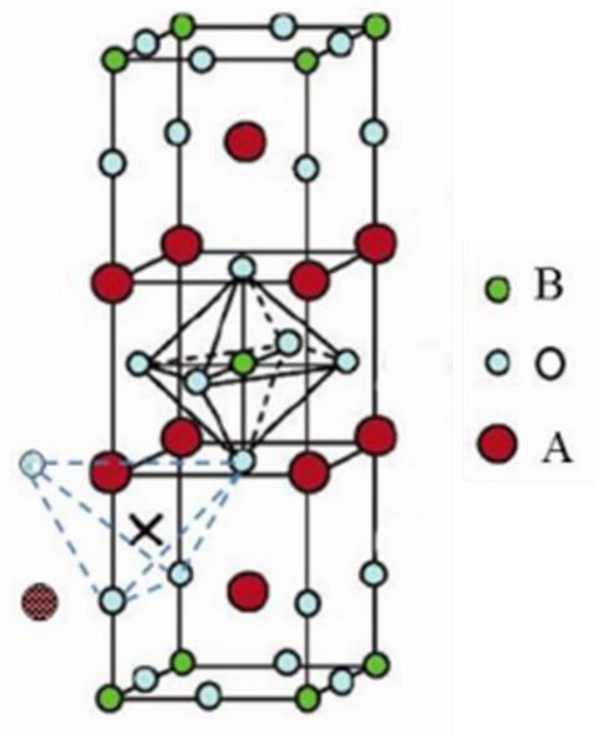

Figure 3.20 The crystal structure of $\mathrm{A}_{2} \mathrm{BO}_{4}(*)$ represent the interstitial oxygen atomics

Table 3.2 Refined cell parameters and error value in I4/mmm space group

\begin{tabular}{|l|l|l|l|l|l|l|}
\hline & $\mathrm{a}$ & $\mathrm{b}$ & $\mathrm{c}$ & $\mathrm{R}_{\mathrm{wp}}(\%)$ & $\mathrm{R}_{\mathrm{p}}(\%)$ & $\chi^{2}$ \\
\hline Pure LNO & 3.86127 & 3.86127 & 12.68641 & 6.39 & 4.68 & 1.689 \\
\hline LNO-LSCF & 3.86768 & 8.36768 & 12.69597 & 6.64 & 4.96 & 1.541 \\
\hline
\end{tabular}

Table 3.3 Refined atomic position for pure LNO in I4/mmm space group

\begin{tabular}{|c|c|c|c|c|c|}
\hline & $\mathrm{x}$ & $\mathrm{y}$ & $\mathrm{z}$ & occupy & UISO \\
\hline La1 & 0.00000 & 0.00000 & 0.36058 & 1 & 0.01147 \\
\hline Ni1 & 0.00000 & 0.00000 & 0.00000 & 1 & 0.01131 \\
\hline O1 & 0.00000 & 0.50000 & 0.00000 & 0.9432 & 0.02833 \\
\hline O2 & 0.00000 & 0.00000 & 0.16961 & 0.9832 & 0.02833 \\
\hline & $*$ & & & & \\
\hline O3 & 0.74087 & 0.00000 & 0.15985 & 0.154 & 0.06283 \\
\hline
\end{tabular}

Table 3.4 Refined atomic position for pure LNO in I4/mmm space group

\begin{tabular}{|c|c|c|c|c|c|}
\hline & $\mathrm{x}$ & $\mathrm{y}$ & $\mathrm{z}$ & occupy & UISO \\
\hline La1 & 0.00000 & 0.00000 & 0.36058 & 1 & 0.01147 \\
\hline Ni1 & 0.00000 & 0.00000 & 0.00000 & 1 & 0.01131 \\
\hline O1 & 0.00000 & 0.50000 & 0.00000 & 0.9432 & 0.02833 \\
\hline
\end{tabular}




\begin{tabular}{|c|c|c|c|c|c|}
\hline O2 & 0.00000 & 0.00000 & 0.16961 & 0.9832 & 0.02833 \\
\hline & $*$ & & & & \\
\hline O3 & 0.74087 & 0.00000 & 0.15985 & 0.154 & 0.06283 \\
\hline
\end{tabular}

Table 3.5 Refined band length in I4/mmm space group

\begin{tabular}{|l|l|l|l|l|l|}
\hline & La-O1 (4) & La-O2 (1) & La-O2 (4) & Ni-O1 (4) & Ni-O2 (2) \\
\hline Pure LNO & 2.618 & 2.423 & 2.757 & 1.931 & 2.153 \\
\hline LNO-LSCF & 2.625 & 2.366 & 2.769 & 1.934 & 2.207 \\
\hline
\end{tabular}

Table 3.6 The valence of transition metal and the amount of nonstoichiometric oxygen in

LNO-based materials by iodometric titration

\begin{tabular}{|c|c|c|c|c|}
\hline & $\mathrm{La}_{2} \mathrm{NiO}_{4+\delta}$ & $\mathrm{La}_{1.9} \mathrm{Sr}_{0.1} \mathrm{NiO}_{4+\delta}$ & $\mathrm{La}_{2} \mathrm{Ni}_{0.9} \mathrm{Co}_{0.1} \mathrm{O}_{4+\delta}$ & $\mathrm{La}_{2} \mathrm{Ni}_{0.7} \mathrm{Co}_{0.3} \mathrm{O}_{4+\delta}$ \\
\hline Ni/Co valence & 2.296 & 2.332 & 2.350 & 2.436 \\
\hline Nonstoichiometric Oxygen & 0.148 & 0.116 & 0.175 & 0.218 \\
\hline
\end{tabular}

3.3.6 Long-term stability and Degradation mechanisms

Fig. 3.21 shows the voltage of the test cells as a function of time at a constant current density of $250 \mathrm{~mW} \cdot \mathrm{cm}^{-2}$ at $750^{\circ} \mathrm{C}$. The obviously higher voltage achieved by using LNOinfiltrated LSCF, as compared to pure LSCF cathode, further confirm that the LNO nanoparticles can serve as high-performance electro-catalyst. However, both of them have experienced a degradation of voltage with the extension of time. The degradation of each cell was calculated by equation $\frac{V_{\text {final }}-V_{\text {peak }}}{V_{\text {peak }}} \times 100 \%$. In the case of bare LSCF cathode, the voltage drops from $0.841-0.839$ to $0.839-0.836 \mathrm{~V}$, showing a degradation rate of $0.36 \%$ over $500 \mathrm{~h}$. This is lower than the values reported in the literature (e.g. 0.9-1.5\% per 1,000 hour) for LSCF cathodes operated under similar conditions $\left[{ }^{123}\right]$. The relatively high stability of pure LSCF cathode provides us a good baseline and high standard for the study of LNO infiltration.

In contrast, the cell using LNO-infiltrated LSCF cathode exhibits performance degradation of about $0.39 \%$ with a voltage drop from0.952-0.949 to $0.949-0.946 \mathrm{~V}$ after $500 \mathrm{~h}$. Thus, a 
slightly higher degradation rate is obtained for LNO-infiltrated LSCF cathode than LSCF itself. Compared to the reports of LSCF based cathodes including modified LSCF with other surface catalysts, as showed much higher degradation rate of the cathodes, the LNOinfiltrated LSCF cathodes with good long-term stability over $500 \mathrm{~h}$ seems to be promising candidates for SOFC application. As far as the similar degradation rate for both LSCF and LNO-infiltrated LSCF cathodes is concerned, the extended durability measurements will be carried out in the future.

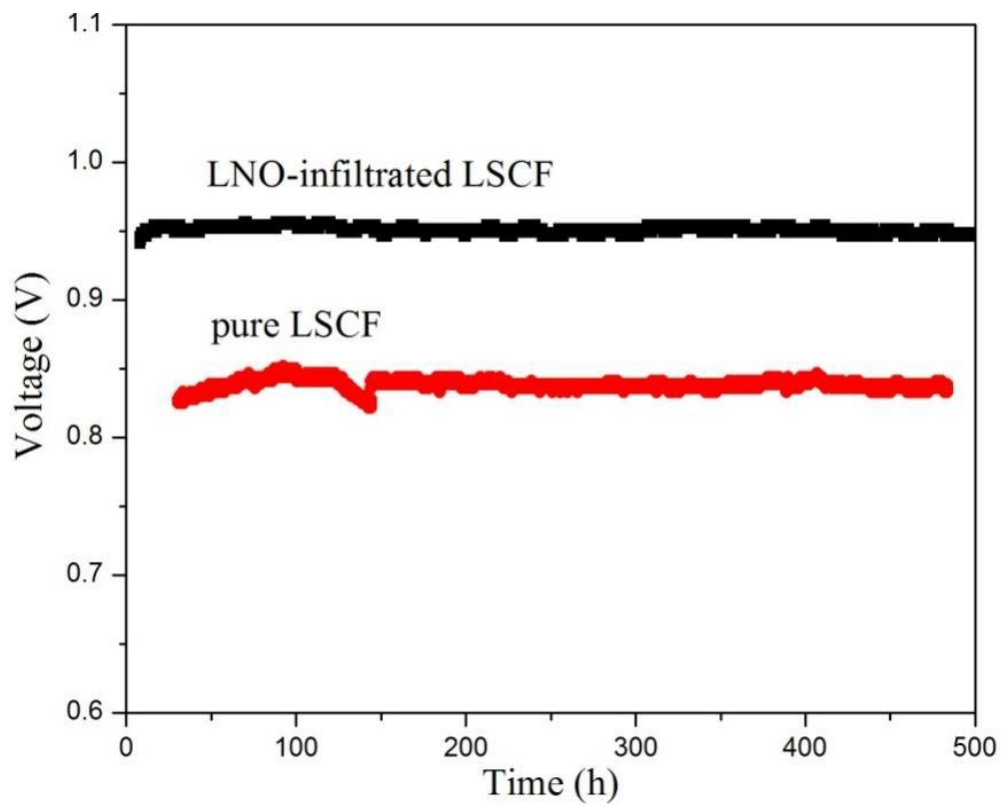

Figure 3.21 Cell voltage as function of time at a constant current density of $250 \mathrm{~mA} \mathrm{~cm}^{-2}$ for both bare LSCF and LNO-infiltrated LSCF

It has to be emphasized that the degradations observed for bare LSCF and LNO-infiltrated LSCF cathodes should be ascribed to different mechanisms. The performance loss with time for the bare LSCF could be associated with cation segregation, like the diffusion of $\mathrm{Sr}$ and La into cathode surface, as widely suggested in the literature. While nanoparticle thermal coarsening or low-performance phase formation resulted from chemical instability between bulk substrate and surface catalyst is usually responsible for long-term operation degradation of cathode containing surface catalysts. In order to explain relatively higher degradation rate of LNO-infiltrated LSCF cathode, the SEM, TEM and EDAX characterization analyses after the on-cell testing are depicted in Fig.3.14 and Fig. 3.22. It can be observed that the growth of LNO nanoparticles from around 50nm to 100-150 nm 
occur and LNO nanoparticles are delaminate from LSCF backbone. Correspondingly after on-cell testing of $500 \mathrm{~h}$, the loss of interface, surface area and $2 \mathrm{~PB} / 3 \mathrm{~PB}$ active regions during the intermediate-temperature measurements could be responsible, at least in part, for the performance degradation the LNO-infiltrated LSCF cathode.

At the same time, the surface chemistry states of LNO-infiltrated LSCF have been investigated to study the degradation mechanism of LNO-infiltrated LSCF further by XPS. Figure 3.23 shows the La $3 \mathrm{~d}$ and $\mathrm{Ni} 3 \mathrm{p}$ spectra on the surface of LNO-coated LSCF samples. The La $3 d$ peak were fitted by at least two sets of La $3 d 5 / 2$ and $3 \mathrm{~d} 3 / 2$ doublets with an energy separation of $1.79 \mathrm{eV}$ and a branching ration of 0.67 . And $\mathrm{Ni} 3 p$ were also fitted by at least two sets of Ni3p 3/2 and 3p1/2 doublets. They represent the surface and bulk chemistry state of $\mathrm{La}$ and $\mathrm{Ni}$. Based on the area of peaks, the ratio of $\mathrm{La}$ and $\mathrm{Ni}$ was calculated. The atomic percentages were calculated according to

$$
\text { Atomic } \%=\frac{I_{a} / S_{a}}{\sum I_{m} / S_{m}}
$$

Where $I$ is the peak intensity and $S$ is the relative elemental sensitivity factor for the Auger line used. The ratio of $\mathrm{La}$ to $\mathrm{Ni}$ changes from 2.25 to 3.94 after $0.2 \mathrm{~V}$ cathodic polarization. It needs to be mentioned that the calculated $\mathrm{La} / \mathrm{Ni}$ ratio may larger than the actual value, because the La 3d peaks area used in calculation contains a part of Ni $2 \mathrm{p}$ peaks except for the real La $3 \mathrm{~d}$ peaks. But the variation tendency of $\mathrm{La} / \mathrm{Ni}$ still indicates that lanthanum enrichment exists on the surface after cathodic polarization treatment, which occupies the surface efficient site instead of active nickel elements. It is possible another reason for the degradation of LNO-infiltrated LSCF cathode. 

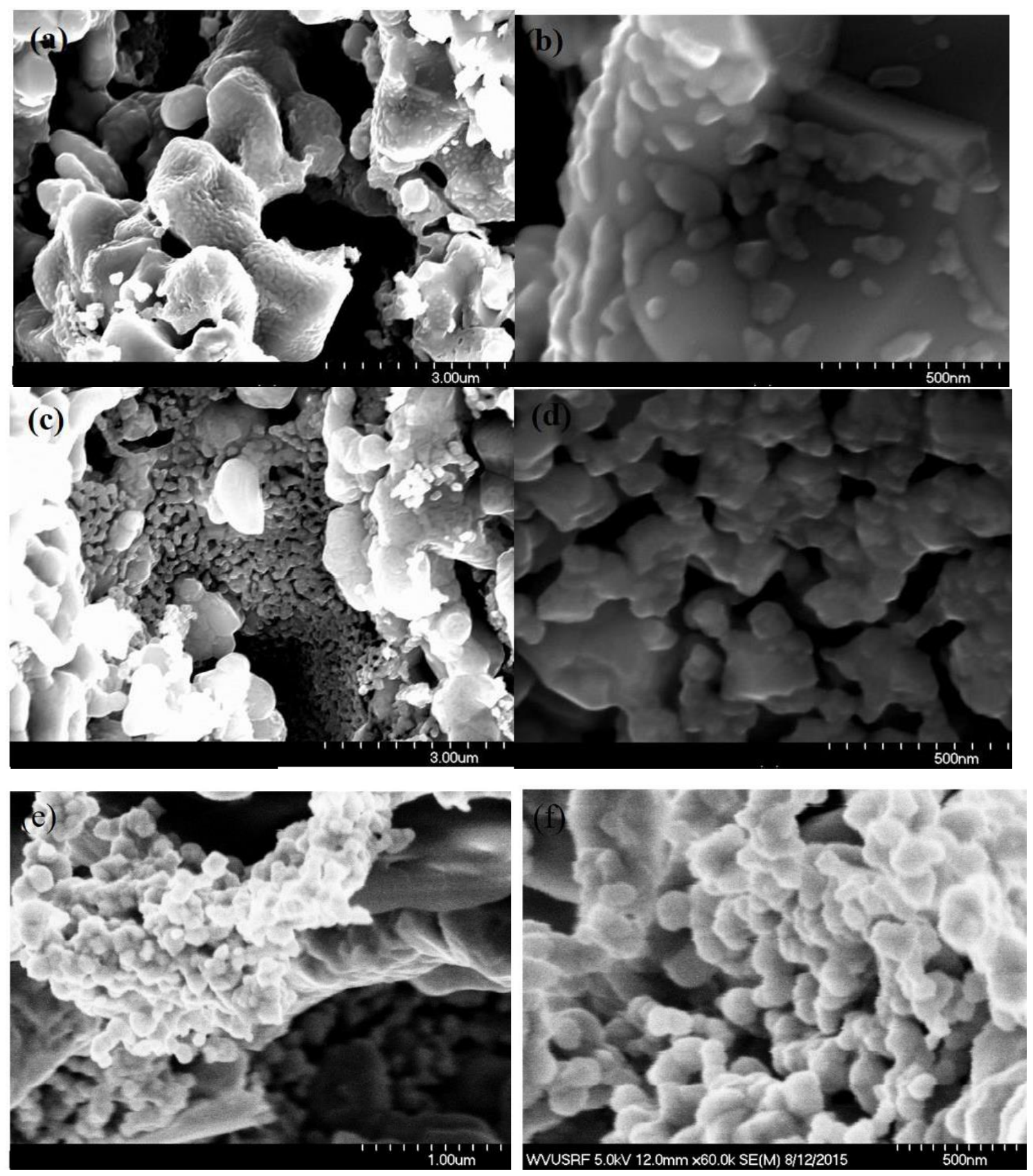

Figure 3.22 SEM image of LSCF cathode with LNO infiltration (a) and (b) without longterm operation; (c) and (d) with $1000 \mathrm{~h}$ long-term heat treatment at $750^{\circ} \mathrm{C}$; (e) and (f) with $500 \mathrm{~h}$ long-term operation under a constant current density of $250 \mathrm{~mA} \cdot \mathrm{cm}^{-2}$ 

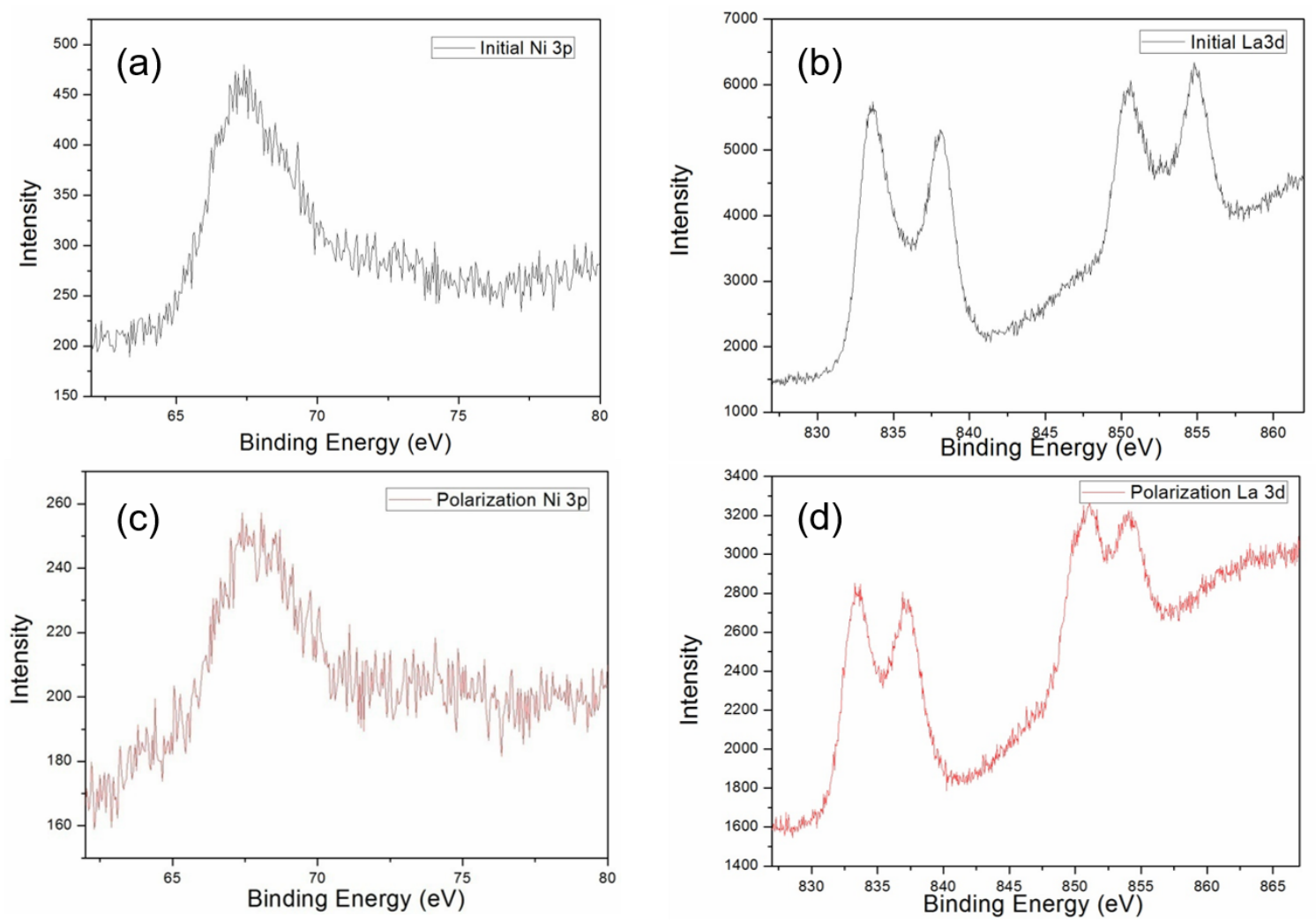

Figure 3.23 Ni 3p and La 3d XPS spectra of $\mathrm{La}_{2} \mathrm{NiO}_{4+\delta}$-coated LSCF (a) and (b) fresh surface; (c) and (d) surface after polarization

\subsection{Summary}

The LNO nanoparticles can be successfully infiltrated into LSCF backbone to obtain a new SOFC cathode with improved ORR kinetics. The currently comparative study of LSCF and LNO-infiltrated LSCF shows a significant reduction in polarization resistance and a $67 \%$ increase in maximum power density by using LNO-infiltrated LSCF cathode at $750^{\circ} \mathrm{C}$. A power density of $637 \mathrm{~mW} \cdot \mathrm{cm}^{-2}$ for the fuel cell using LNO-infiltrated LSCF cathode can be still achieved after long-term durability of about $500 \mathrm{~h}$ at $750^{\circ} \mathrm{C}$, compared with the power density of $392 \mathrm{~mW} \cdot \mathrm{cm}^{-2}$ for the baseline LSCF sample. The LNO-infiltrated LSCF materials can act as a more high active surface oxygen exchange cathode with promising electrochemical properties and fast ORR behavior. . Both ECR and LSCF self-infiltrated electrode results indicate that the performance improvement also results from the intrinsic LNO surface/ hetero-structured LSCF/LNO interface architecture offering more active 
reaction regions of $2 \mathrm{~PB}$ and $3 \mathrm{~PB}$, except for nanoscale $\mathrm{LNO}$ fine particles increasing surface active area. In consideration of relatively narrow gap between LSCF-infiltrated LSCF and LNO-infiltrated LSCF electrode resistance, the largest driving force for the increased performance may relate to the microstructure change with LNO fine particles introducing. Meanwhile, the cation segregation of LSCF could facilitate the surface catalytic properties and ORR kinetics of the LNO-infiltrated LSCF cathode with respect to favorable acceptor of LNO from $\mathrm{Sr} / \mathrm{Co}$ doping. Although particle coarsening of LNO and cation diffusion from LSCF are observed, the preliminary stability results show a good long-term stability of the LNO-infiltrated LSCF cathode with a low degradation rate of $0.39 \%$ over $\sim 500 \mathrm{~h}$ at a constant current density of $250 \mathrm{~mA} \cdot \mathrm{cm}^{-2}$. The weak $\mathrm{Sr}$ peaks before and after polarization demonstrate the inhibition of LNO for $\mathrm{Sr}$ segregation. The nanoparticles aggregation/growth and lanthanum enrichment on surface can be observed in SEM images, which decrease either the effective surface reaction area or heterogeneous interface area. Therefore, to further enhance the long-term stability of infiltrated ( $\left.\mathrm{La}_{0.6} \mathrm{Sr}_{0.4}\right)_{0.95} \mathrm{Co}_{0.2} \mathrm{Fe}_{0.8} \mathrm{O}_{3-\delta}$ (LSCF) cathode, two kinds of new hetero-structured cathodes, $\mathrm{CeO}_{2} \&$ LNO co-infiltrated LSCF and $\mathrm{La}_{2-x} \mathrm{NiO}_{4+\delta}$-infiltrated LSCF, have been designed and characterized in next work. 


\section{Chapter 4: Effect of A-site Deficient $\mathrm{La}_{2} \mathrm{NiO}_{4+\delta}$ on Stability of Hetero-structured Cathode}

\subsection{Introduction}

In this work, the A-site deficient $\mathrm{LNO}$ were prepared in our recent experiment to inhibit the lanthanum segregation from LNO that dominates the surface kinetic activity of these $\mathrm{A}_{n+1} \mathrm{~B}_{\mathrm{n}} \mathrm{O}_{3 n+1}$ structures materials after long-term operation $\left[{ }^{219,220}\right]$. The absence of nickel were detected after polarization treatment on LNO/LSCF surface reported in previous work chapter 3. And furthermore, it has been recently demonstrated that surface segregation takes place in a very short time for a number of different $\mathrm{A}_{\mathrm{n}+1} \mathrm{~B}_{\mathrm{n}} \mathrm{O}_{3 \mathrm{n}+1}$ electrode materials, even at low temperatures, giving rise to structural rearrangements [135,221-223]. A similar absence of $\mathrm{Ni}$ and $\mathrm{Ni}$ : La non-stoichiometry was obtained in these RP phase surface. No Ni was found in the low energy ion scattering (LEIS) spectra of the surfaces within the detection limit $\left.{ }^{224}\right]$. The $\mathrm{Ni}$ : $\mathrm{La}(\mathrm{Sr})$ ratio was also found to be far from stoichiometry down to a depth of about $7 \mathrm{~nm}$. This predominant surface structure implies that the transition metals (B site cations), usually invoked as the active sites for the interaction with the gaseous oxygen species, are hidden below the first atomic surface layers, and thus, their presence at the outermost surface does not seem to directly control the catalytic activity of these materials. The recent simulation work carried out by $\mathrm{Wu}$ et al. $\left[{ }^{225}\right]$ has provided some plausible answers to the open question of why $\mathrm{Ni}$ is absent from the surface of $\mathrm{La}_{2} \mathrm{NiO}_{4+\delta}$. The authors found that the decomposition of $\mathrm{La}_{2} \mathrm{NiO}_{4}$ to produce $\mathrm{La}_{2} \mathrm{O}_{3}$ and higher-order RP phases is thermodynamically favorable and would lead to a La-terminated and Ni-deficient surface. Therefore, two possible ways were considered to solve this problem. One is adjustment of elements composition to form more stable layered perovskite $\mathrm{K}_{2} \mathrm{NiF}_{4}$ phases based on the phase diagram calculation. Another one is simply introduce La deficient in the stoichiometry LNO infiltration solution to inhibit the formation of lanthanum oxide, which was widely used in perovskite materials (LSCF/LSC) to suppress the cations segregation. The latter way was tried out in this work.

Meanwhile, the A-site deficient introduction has another advantage on the electronic conductivity of LNO materials. It is well known that even though LNO-based materials 
exhibit high oxygen bulk diffusion and surface exchange coefficient, its lower electronic conductivity, as one of key factors, still limits the practical use as SOFC cathode [ ${ }^{226-228}$ ]. To improve the electronic conductivity of LNO, lower valence element, such as $\mathrm{Sr}$ and $\mathrm{Ca}$, is often introduced on A-site to produce extra electronic holes as charge compensation. Based on the similar charge compensation mechanism, the deficiency of trivalent lanthanum could produce more $\mathrm{Ni}^{3+}$ and less interstitial oxygen defects. The increase of $\mathrm{Ni}^{2+}-\mathrm{O}-\mathrm{Ni}^{3+}$ can improve the total conductivity of the materials.

Based on these two points, in this work, the La-deficiency was introduced into the stoichiometry LNO infiltration solution. A-site deficient LNO has the possibility to enhance the electronic conductivity and inhibit lanthanum segregation from LNO. A-site deficient LNO cathode materials were prepared with different concentration of deficiency. The effects of A-site deficient on the lattice structure, electronic conductivity, the electrochemical performance were detected to confirm the intrinsic properties of A-site deficient LNO, and the possible ORR mechanisms were discussed. Then the electrochemical performance and stability of A-site deficient LNO infiltrated/coated LSCF, especially the surface composition in LNO, were systematically investigated.

\subsection{Experimental}

$\mathrm{La}_{2-\mathrm{x}} \mathrm{NiO}_{4}(\mathrm{x}=0-0.2)$ powders were fabricated by sol-gel method. Stoichiometric nitrates $\mathrm{La}\left(\mathrm{NO}_{3}\right)_{3} \cdot 6 \mathrm{H}_{2} \mathrm{O}$ and $\mathrm{Ni}\left(\mathrm{NO}_{3}\right)_{2} \cdot 6 \mathrm{H}_{2} \mathrm{O}$ (Alfa Aesar) were dissolved into distilled water separately with complexant citric acid (Alfa Aesar). The ratio of citric acid to metal ions in sol-gel solution is 1.2 . The mixed solution was held at $80^{\circ} \mathrm{C}$ and stirred by magneton until gelation. The gel was heated up to $400^{\circ} \mathrm{C}$ to decompose the nitrates and residual organics. The resultant powders were then sintered at $1100^{\circ} \mathrm{C}$ for $4 \mathrm{~h}$ in air. Phase purity and the limitation of A-site deficient in LNO were examined by X-ray diffraction (XRD) patterns and the detail information of the crystal structure was analyzed by GASA XRD refinement software. The concentration of oxygen defect in different A-site deficient LNO materials was detected by the iodometric titration method.

The conductivity testing is tested by Van der pauw methods. The dense A-site deficient LNO pellets for conductivity measurement were dry pressed using dies with the diameters 
of $17 \mathrm{~mm}$ followed by sintering at $1300^{\circ} \mathrm{C}$ for $4 \mathrm{~h}$. Four silver wires were bonded to the surface of samples using silver paste symmetrically, followed by heating to $750^{\circ} \mathrm{C}$. The measurement was carried out in a temperature-programed tube furnace. The electrical conductivity data was collected using Keithley, with sourcing current of $10 \mathrm{~mA}$ and monitoring the voltage.

A symmetric cell configuration of $\mathrm{La}_{2-\mathrm{x}} \mathrm{NiO}_{4}|\mathrm{GDC}| \mathrm{YSZ}|\mathrm{GDC}| \mathrm{La}_{2-\mathrm{x}} \mathrm{NiO}_{4}$ were assembled with the similar processes described in chapter 3. Finally, the green symmetrical electrodes were also co-fired at $1150^{\circ} \mathrm{C}$ for $3 \mathrm{~h}$ with an active surface area of about $0.7 \mathrm{~cm}^{2}$. The electrochemical performance of $\mathrm{La}_{2} \mathrm{NiO}_{4}$ were characterized by EIS under OCV conditions. For comparison and performance evaluation, $\mathrm{La}_{2-\mathrm{x}} \mathrm{NiO}_{4}$ was infiltrated into the both LSCF sides in the LSCF|GDC|YSZ|GDC|LSCF cell, as described in chapter 3, to form a symmetric cell using $\mathrm{La}_{2-x} \mathrm{NiO}_{4}$-infiltrated LSCF cathode. Silver rings were attached to both electrode sides of the symmetric cells by using Pt paste as current collectors.

To avoid the influence of microstructure, the dense $\mathrm{La}_{2-x} \mathrm{NiO}_{4}$ was coated on LSCF surface to identify the surface elements composition by air brush method. In this process, $\left(\mathrm{La}_{0.6} \mathrm{Sr}_{0.4}\right)_{0.95} \mathrm{Co}_{0.2} \mathrm{Fe}_{0.8} \mathrm{O}_{3-\delta}$ and $\mathrm{La}_{2-\mathrm{x}} \mathrm{NiO}_{4+\delta}(\mathrm{x}=0-0.2)$ powders were added into ethanol $(5 \mathrm{~g}$ powders to $30 \mathrm{ml}$ ethanol) separately along with polyvinyl butyral (PVB) and fish oil at the weight ratio of 100:2:1.5, then ball-milled for $24 \mathrm{~h}$ to produce suspensions which were orderly applied onto one sides of electrolyte to prepare dense cathode layers. The electrolyte GDC green pellets $(2.5 \mathrm{~g}$ ) were prepared by dry pressing method in the die with diameter $16.5 \mathrm{~mm}$ under $50 \mathrm{MPa}$. The certain volume suspensions were evenly sprayed onto the surface of green electrolyte pellets, followed by a final pressing of $200 \mathrm{MPa}$. The green hetero-structured pellets $\left(\mathrm{GDC}|\mathrm{LSCF}| \mathrm{La}_{2-\mathrm{x}} \mathrm{NiO}_{4}\right)$ were sintered up to $1300^{\circ} \mathrm{C}$ for $4 \mathrm{~h}$ to obtain dense pellets. To avoid any possible initial element enrichment on the surface, the sintered pellets were polished manually using sandpaper then $2 \mu \mathrm{m}$ polishing cloth. Pt paste was applied on the opposite side to form the counter electrode and a silver ring was fixed on the middle of electrode cylindrical surface. Silver rings were attached to both electrode sides of the symmetric cells by using Pt paste as current collectors.

The electrochemical impedance spectroscopy (EIS) measurements for the symmetrical cells were carried out by an Auto lab station (AUT85484) in ambient air under open circuit 
voltage $(\mathrm{OCV})$ with or without $-0.2 \mathrm{~V}$ polarization. The frequency range was within 0.01 to $100 \mathrm{kHz}$ at an amplitude of alternating circuit (AC) signal of $10 \mathrm{mV}$, and the measured temperature was controlled from 550 to $750^{\circ} \mathrm{C}$. All impedance data were collected after keeping the desired temperature for more than $30 \mathrm{~min}$.

\subsection{Results and Discussion}

\subsubsection{Phase Composition and Structure}

Fig. 4.1 gives the XRD patterns of as-prepared A-site deficient LNO powders. The patterns confirmed the existence of single Ruddlesden-Popper phase (PDF\# 34-0314) for $\mathrm{x}<0.2$, and no detectable perovskite $\mathrm{LaNiO} 3$ phase shows in the XRD patterns. However, for the sample $\mathrm{La}_{1.80} \mathrm{NiO}_{4+\delta}$, a great amount of impurity peaks is visible in the XRD pattern. Therefore, the lanthanum deficiency limit is $x<0.2$ in $\mathrm{La}_{2-x} \mathrm{NiO}_{4+\delta}$. To give an insight into the specified information of $\mathrm{La}_{2-x} \mathrm{NiO}_{4+\delta}$ crystal structure, GASA refinement was performed on the XRD data of the A-site deficient LNO. A shifted Cherbyshev background function and pseudo Voigt peak shape were used in the refinement process. A best fit to the experimental data was obtained in the tetragonal $\mathrm{I} / \mathrm{mmm}$ space group with the $\mathrm{La}$ occupying $(0,0, \mathrm{z})$ position, $\mathrm{Ni}$ occupying $(0,0,0)$ position and two kinds of oxygen occupying $(0,0, \mathrm{z})$ and $(0,1 / 2,0)$ position. Refined crystal structure information was shown in Fig. 4.2 and Table 4.1 and 4.2. The lattice parameters $a, b$ and $c$ of samples are listed in Table 4.1. All the lattice parameters increase with an increase in $\mathrm{x}$. The refinement results, as shown in Table 4.2 indicate that the lattice parameters increase is attributed to the distorted octahedron in LNO with the existence of A-site deficiency. For the bond length analysis, the La-O2 (*1) bond length decreases after LNO applied on LSCF surface, which represents the length of $\mathrm{AO}$ rock salt layer which is critical for the oxygen interstitial transport located in the oxygen or lanthanum tetrahedron. The decrease of La-O2 $(* 1)$ will reduce the space of interstitial oxygen transport channels and further influence the oxygen transport properties. 


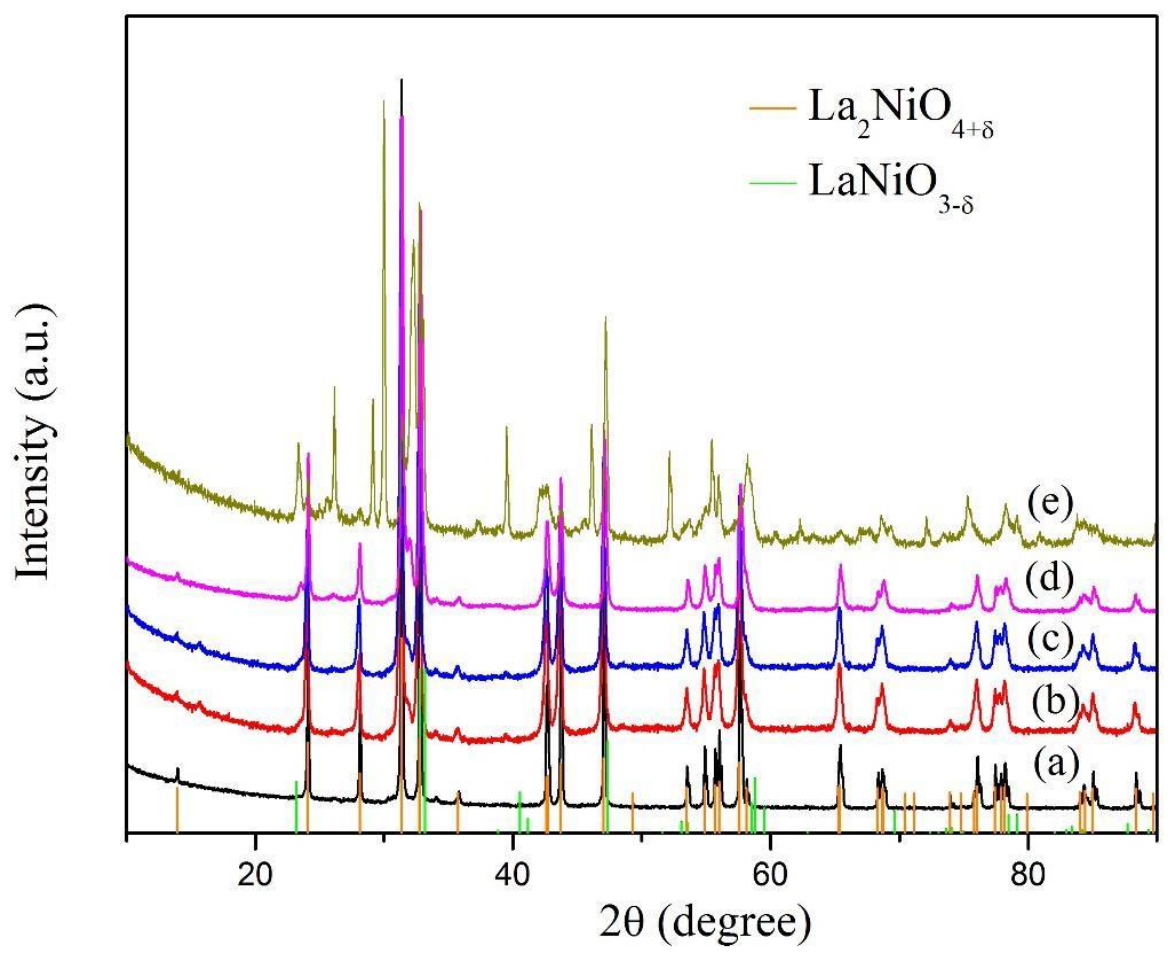

Figure 4.1 XRD pattern of $\mathrm{La}_{-x} \mathrm{NiO}_{4+\delta}(\mathrm{x}=0-0.2)$ powders after sintering $1000^{\circ} \mathrm{C}$ for $6 \mathrm{~h}$ in air: (a) $\mathrm{x}=0$; (b) $\mathrm{x}=0.05$; (c) $\mathrm{x}=0.10$; (d) $\mathrm{x}=0.15$; (e) $\mathrm{x}=0.2$.

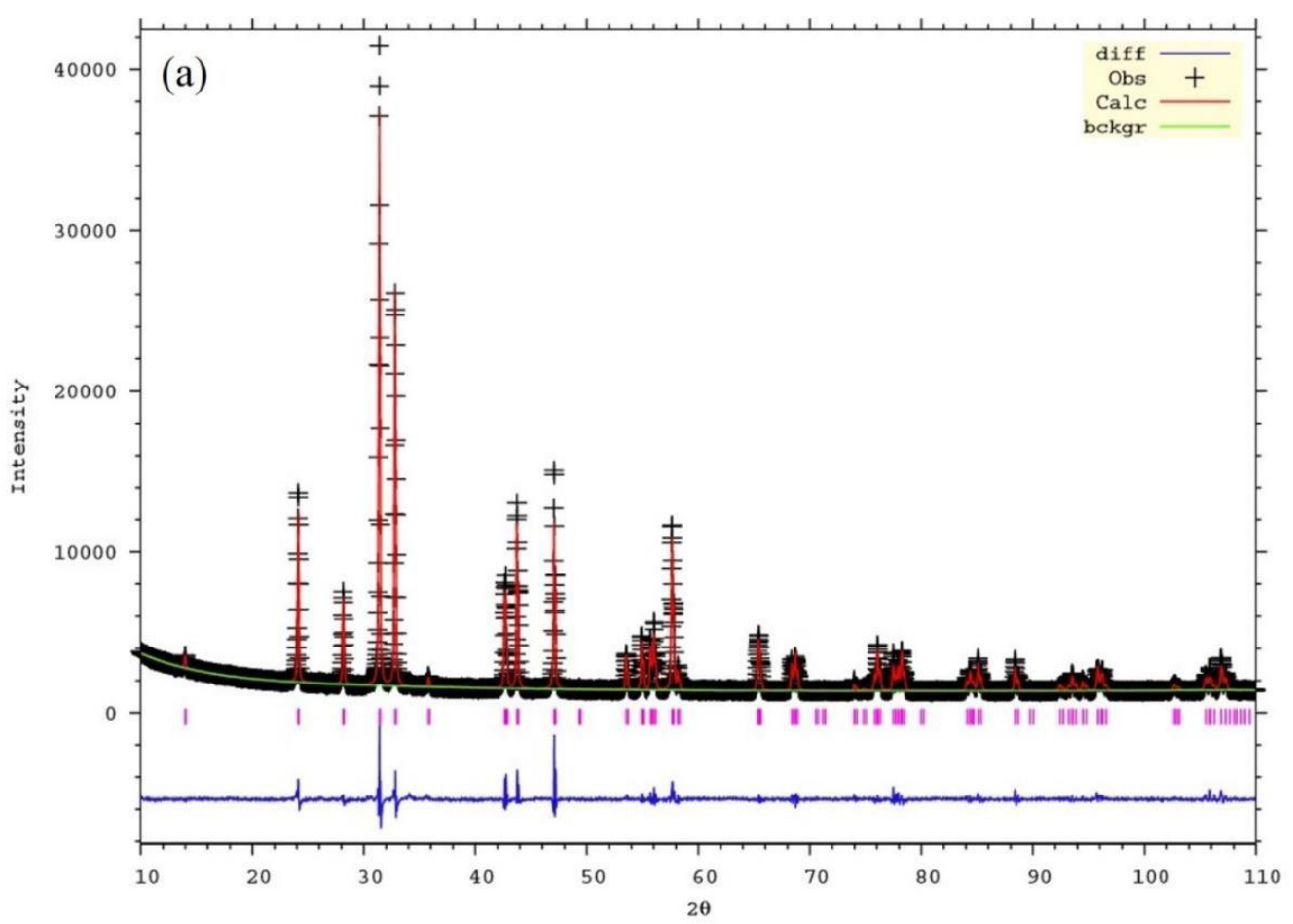



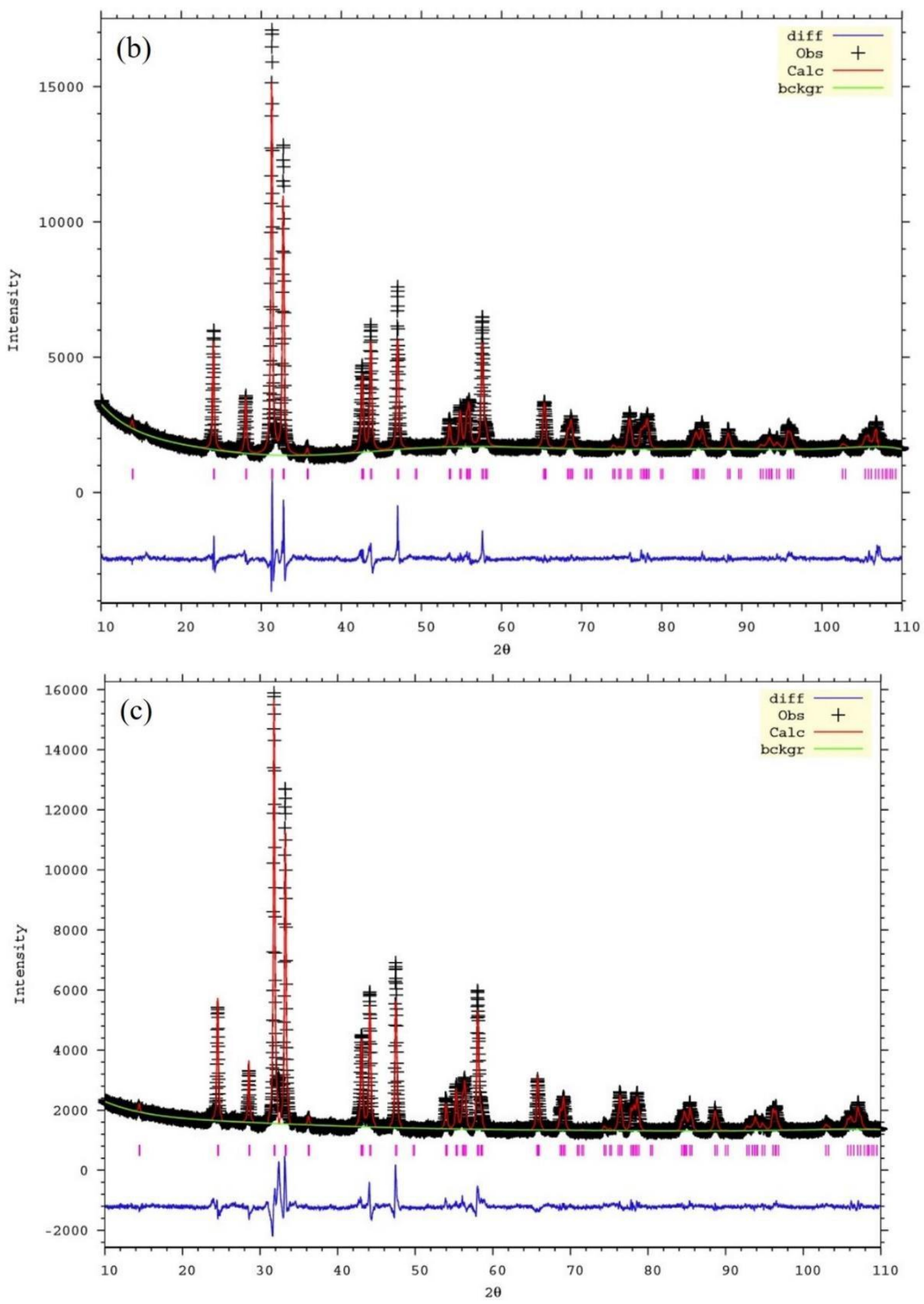


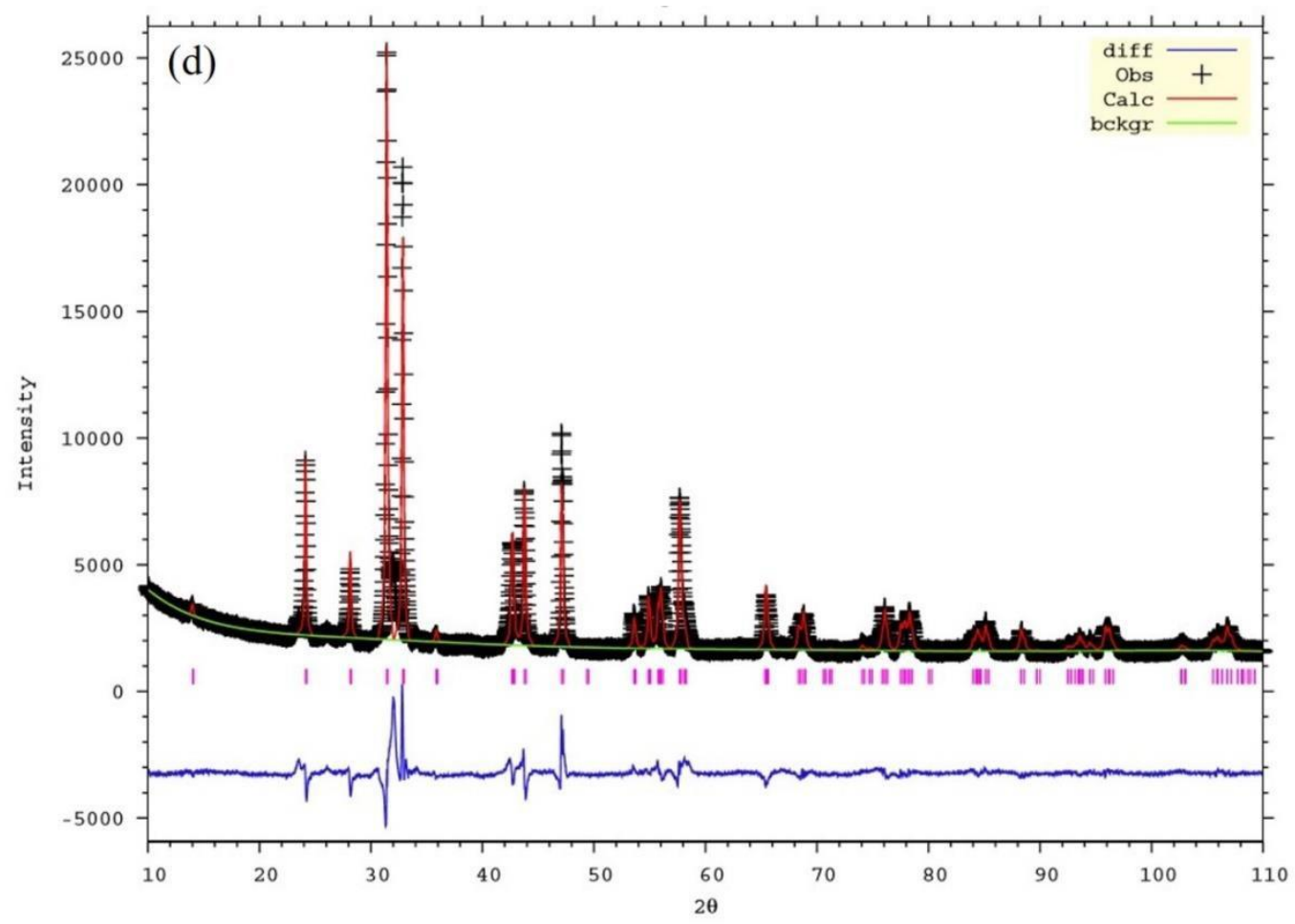

Figure 4.2 Rietveld refinement profiles of A-site deficient $\mathrm{La}_{2-\mathrm{x}} \mathrm{NiO}_{4+\delta}$ after being sintered at $1100^{\circ} \mathrm{C}$ (space group I4/mmm): (a) $\mathrm{x}=0$; (b) $\mathrm{x}=0.05$; (c) $\mathrm{x}=0.10$; (d) $\mathrm{x}=0.15$. (+) represent the experimental data points, solid red line is refined data fit

Table 4.1 Refined cell parameters and error value of A-site deficient LNO in I4/mmm space group

\begin{tabular}{|l|l|l|l|l|l|l|}
\hline & $a$ & $b$ & $c$ & $R_{w p}(\%)$ & $R_{p}(\%)$ & $\chi^{2}$ \\
\hline $\mathrm{La}_{2} \mathrm{NiO}_{4+\delta}$ & 3.86215 & 3.86215 & 12.68875 & 5.16 & 3.35 & 5.205 \\
\hline $\mathrm{La}_{1.95} \mathrm{NiO}_{4+\delta}$ & 3.86347 & 3.86347 & 12.70346 & 5.12 & 3.34 & 5.106 \\
\hline $\mathrm{La}_{1.90} \mathrm{NiO}_{4+\delta}$ & 3.86868 & 3.86868 & 12.723816 & 5.90 & 3.69 & 5.946 \\
\hline $\mathrm{La}_{1.85} \mathrm{NiO}_{4+\delta}$ & 3.86518 & 3.86518 & 12.718372 & 7.92 & 4.73 & 13.96 \\
\hline
\end{tabular}

Table 4.2 Refined band length of A-site deficient LNO in I4/mmm space group

\begin{tabular}{|l|l|l|l|l|l|}
\hline & La-O1 (4) & La-O2 (1) & La-O2 (4) & Ni-O1 (4) & Ni-O2 (2) \\
\hline $\mathrm{La}_{2} \mathrm{NiO}_{4+\delta}$ & 2.618 & 2.423 & 2.757 & 1.931 & 2.153 \\
\hline $\mathrm{La}_{1.95} \mathrm{NiO}_{4+\delta}$ & 2.626 & 2.422 & 2.757 & 1.932 & 2.152 \\
\hline
\end{tabular}




\begin{tabular}{|l|l|l|l|l|l|}
\hline $\mathrm{La}_{1.90} \mathrm{NiO}_{4+\delta}$ & 2.631 & 2.379 & 2.767 & 1.934 & 2.199 \\
\hline $\mathrm{La}_{1.85} \mathrm{NiO}_{4+\delta}$ & 2.631 & 2.369 & 2.762 & 1.931 & 2.198 \\
\hline
\end{tabular}

\subsubsection{Conductivity, Oxygen Non-Stoichimetry and Electrochemical Performance}

Fig. 4.3 indicates the electrical conductivities of as-prepared A-site deficient LNO as a function of temperature $\left(250-800^{\circ} \mathrm{C}\right)$. The conductivities of all powders have a similar trend with temperature, the conductivity initially increases and then decreases with temperature. The conductivity transition temperature of $\mathrm{La}_{2-x} \mathrm{NiO}_{4+\delta}$ increases with increasing A-site deficiency, and the conductivities of A-site deficient LNO are more stable than LNO materials under low temperature, both of which could be beneficial to the application at low-temperature for A-site deficient $\mathrm{LNO}$ as cathode materials. The $\mathrm{La}_{2-x} \mathrm{NiO}_{4+\delta}$ with higher deficient exhibits the slightly higher conductivity below $800^{\circ} \mathrm{C}$. The maximum conductivities of $\mathrm{La}_{1.85} \mathrm{NiO}_{4+\delta}, \mathrm{La}_{1.95} \mathrm{NiO}_{4+\delta}$ and $\mathrm{La}_{2} \mathrm{NiO}_{4+\delta}$ are 105, 100 and $97 \mathrm{Scm}^{-1}$, respectively. Focusing on lanthanum deficient in LNO, the charge compensation mechanisms can be represented by two defect equations involving either oxygen vacancy or hole formation. The positive holes are treated in localized terms as $\mathrm{Ni}^{3+}$ and $\mathrm{O}^{-}$small polaron species to form Ni-O-Ni transport way of electrons, which could increase p-type conductivity and promote overall reactivity of the catalyst material. The iodometric titration results shown in Table 4.3 indicate that part of lanthanum deficient was compensated by holes and increase the concentration of $\mathrm{Ni}^{3+}-\mathrm{O}^{-} \mathrm{Ni}^{2+}$ transport way, thereby increasing the electronic conductivity. Meanwhile, the charge introduced by lanthanum deficiency is compensated by an oxygen vacancy increase in perovskite and a reduction of interstitial oxygen in AO salt layer. 


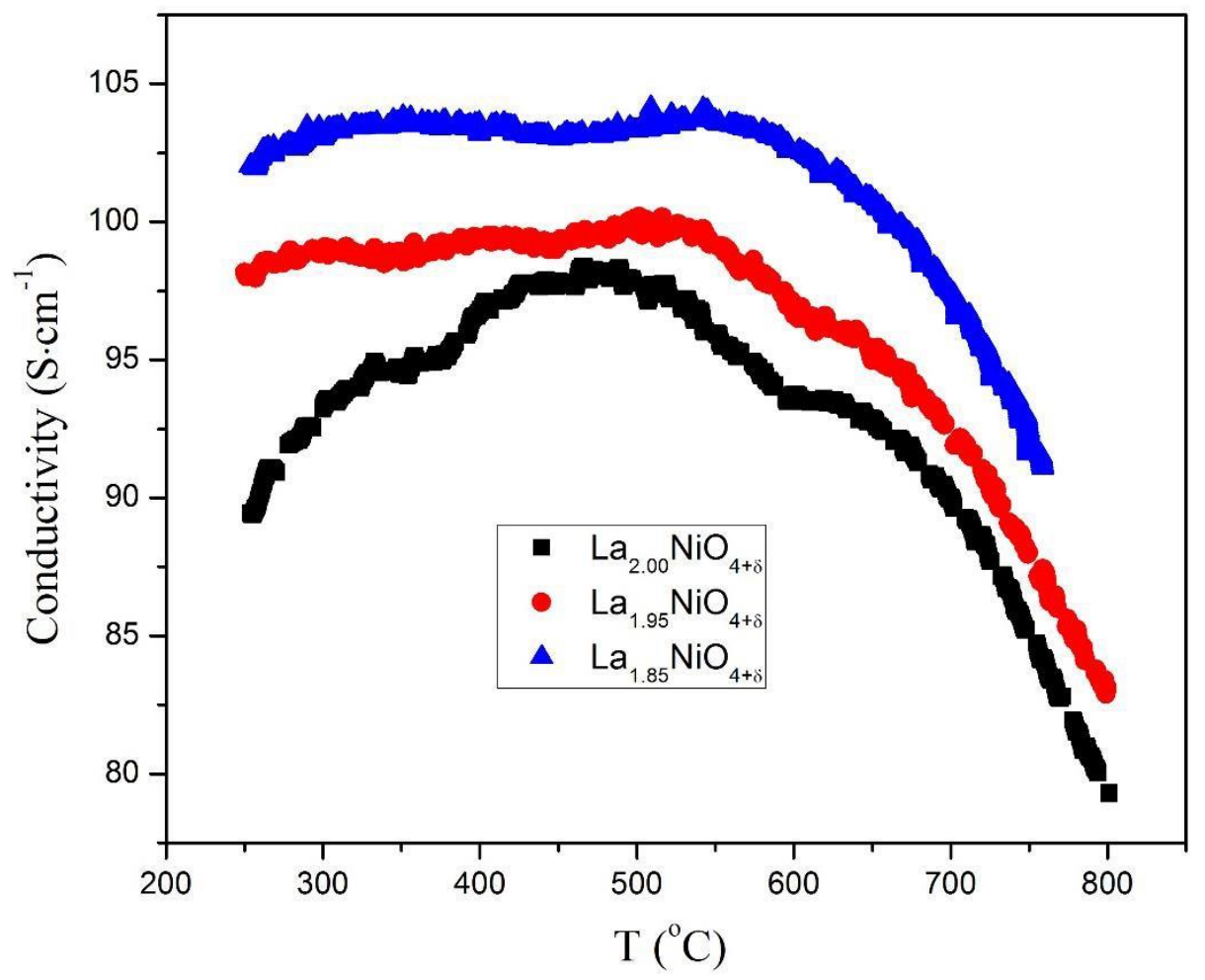

Figure 4.3 The total conductivity of $\mathrm{La}_{2}-\mathrm{NiO}{ }_{4+\delta}$ pellets

Table 4.3 The valence of transition metal and the amount of nonstoichiometric oxygen in A-site deficient LNO materials by iodometric titration

\begin{tabular}{|c|c|c|c|c|}
\hline & $\mathrm{La}_{2} \mathrm{NiO}_{4+\delta}$ & $\mathrm{La}_{1.95} \mathrm{NiO}_{4+\delta}$ & $\mathrm{La}_{1.90} \mathrm{NiO}_{4+\delta}$ & $\mathrm{La}_{1.85} \mathrm{NiO}_{4+\delta}$ \\
\hline $\mathrm{Ni}^{2+} / \mathrm{Ni}^{3+}$ valence & 2.296 & 2.329 & 2.354 & 2.38 \\
\hline Nonstoichiometric Oxygen & 0.148 & 0.0893 & 0.0269 & -0.0350 \\
\hline
\end{tabular}

Representative EIS data for symmetric cells using $\mathrm{La}_{2-x} \mathrm{NiO}_{4+\delta}(\mathrm{x}=0-0.2)$ cathodes collected at $\mathrm{OCV}$ in air at temperature of $750^{\circ} \mathrm{C}$, respectively, are shown in Fig. 4.4. Typical equivalent circuits $\mathrm{R}_{\mathrm{s}}\left(\mathrm{R}_{\mathrm{HF}} \mathrm{Q}_{\mathrm{HF}}\right)\left(\mathrm{R}_{\mathrm{LF}} \mathrm{Q}_{\mathrm{LF}}\right)$ have been used to deconvolute the measured data for the cathodes, where $R_{s}$ is the ohmic resistance of the symmetrical cells, and $R_{n}$ and $Q_{n}$ indicate resistance and constant phase element of different electrode processes, respectively. In the all case of $\mathrm{La}_{2-\mathrm{x}} \mathrm{NiO}_{4+\delta}(\mathrm{x}=0-0.2)$ cathodes, two RQ elements of semi-circle arcs reflect the electrode polarization. A equivalent capacitance value $\operatorname{CHF}\left(\underset{n}{C}=(\underset{n}{Q})^{1 / n} / R\right)$ 
in the range of $10^{-4}-10^{-3} \mathrm{~F} \cdot \mathrm{cm}^{-2}$ are obtained from high frequency response and corresponding to $\mathrm{C}_{\mathrm{LF}}$ value of $\sim 10^{-2} \mathrm{~F} \cdot \mathrm{cm}^{-2}$, both of which is typically attributed to charge transfer process and an intermediate behavior with a similar surface process. The $R_{p}$ values of $\mathrm{La}_{2-\mathrm{x}} \mathrm{NiO}_{4+\delta}(\mathrm{x}=0-0.2)$ cathodes are $0.37,0.42,0.8$ and $1.18 \Omega \cdot \mathrm{cm}^{2}$ at $750{ }^{\circ} \mathrm{C}$, respectively. The increase in cathode polarization resistance demonstrates that lanthanum deficient decreases the electrochemical activity and surface exchange kinetics of LNO electrodes.. Temperature-dependent polarization resistance is characterized in Fig. 4.5. At $550^{\circ} \mathrm{C}$, an additional arc shows in A-site deficient LNO cathode as shown in Fig. 4.6. Considering the difference of oxygen ionic conductivity for electrolyte and $\mathrm{La}_{2-x} \mathrm{NiO}_{4+\delta}$ $(\mathrm{x}=0-0.2)$ materials, the additional arc is proposed to represent the interface charge transfer between electrode and electrolyte. Comparing the Nyquist plots $750^{\circ} \mathrm{C}$ to $550^{\circ} \mathrm{C}$, the high frequency arcs have a larger variation for $\mathrm{La}_{2-\mathrm{x}} \mathrm{NiO}_{4+\delta}(\mathrm{x}=0-0.2)$ cathodes than that of the low frequency arcs, as shown in Fig. 4.7 and 4.8. Combined with reduction of oxygen defect concentration detected by iodometric titration method in Table 4.3, the narrow channel of interstitial oxygen transport will suppress the oxygen incorporation rate on $\mathrm{La}_{2}$ ${ }_{x} \mathrm{NiO}_{4+\delta}$ surface by introduction of lanthanum deficiency. Although the $\mathrm{La}_{2} \times \mathrm{NiO}_{4+\delta}$ with higher deficient have higher conductivity and rapid bulk oxygen transport properties, the surface charge transfer still gradually dominates the impedance with lanthanum deficient increasing. The surface charge transfer process for LNO materials is thus possibly controlled by surface interstitial oxygen concentration along with transition metal states. 


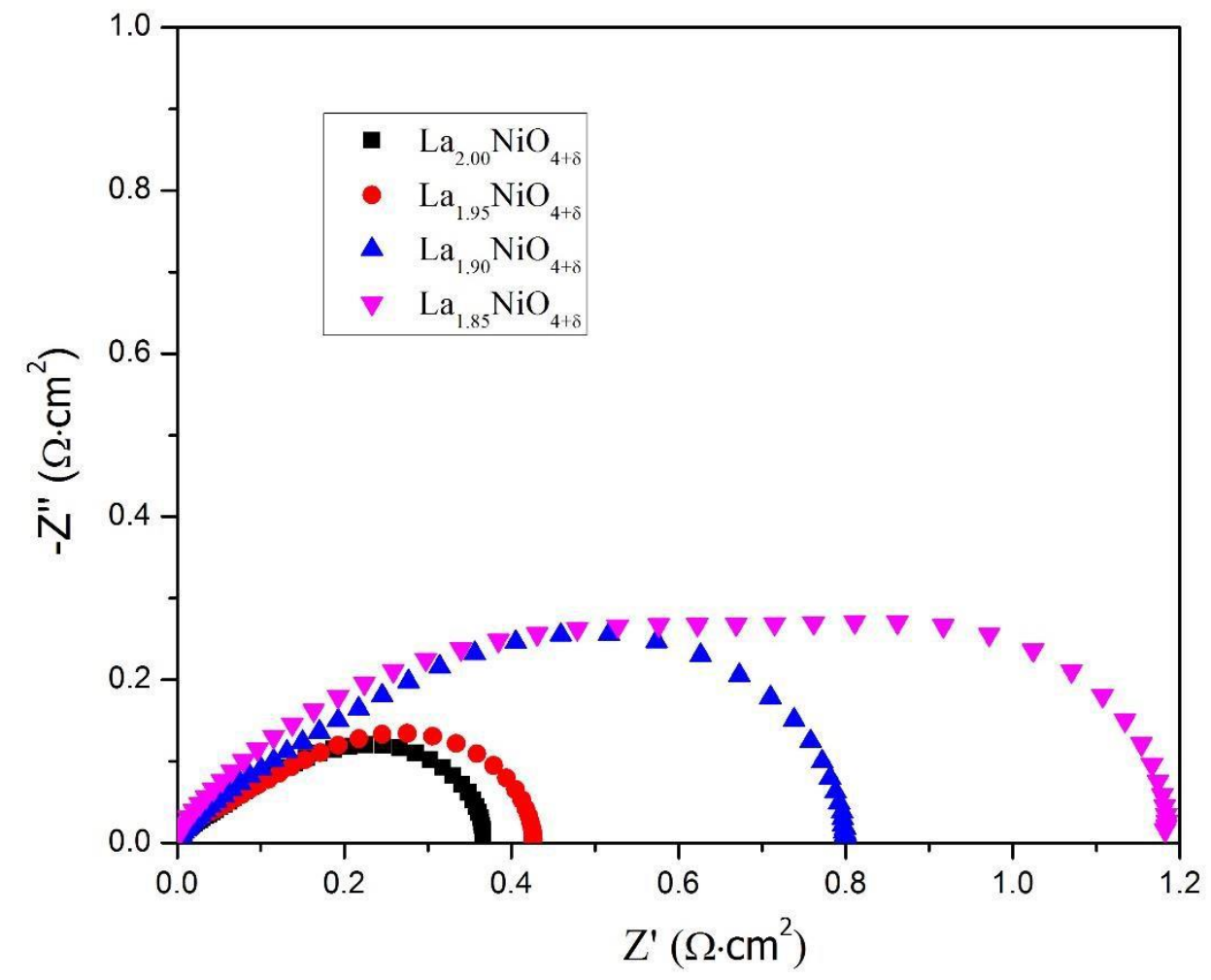

Figure 4.4 Temperature-dependent polarization cathode resistance of $\mathrm{La}_{2-\mathrm{x}} \mathrm{NiO}_{4+\delta}(\mathrm{x}=0-0.2)$

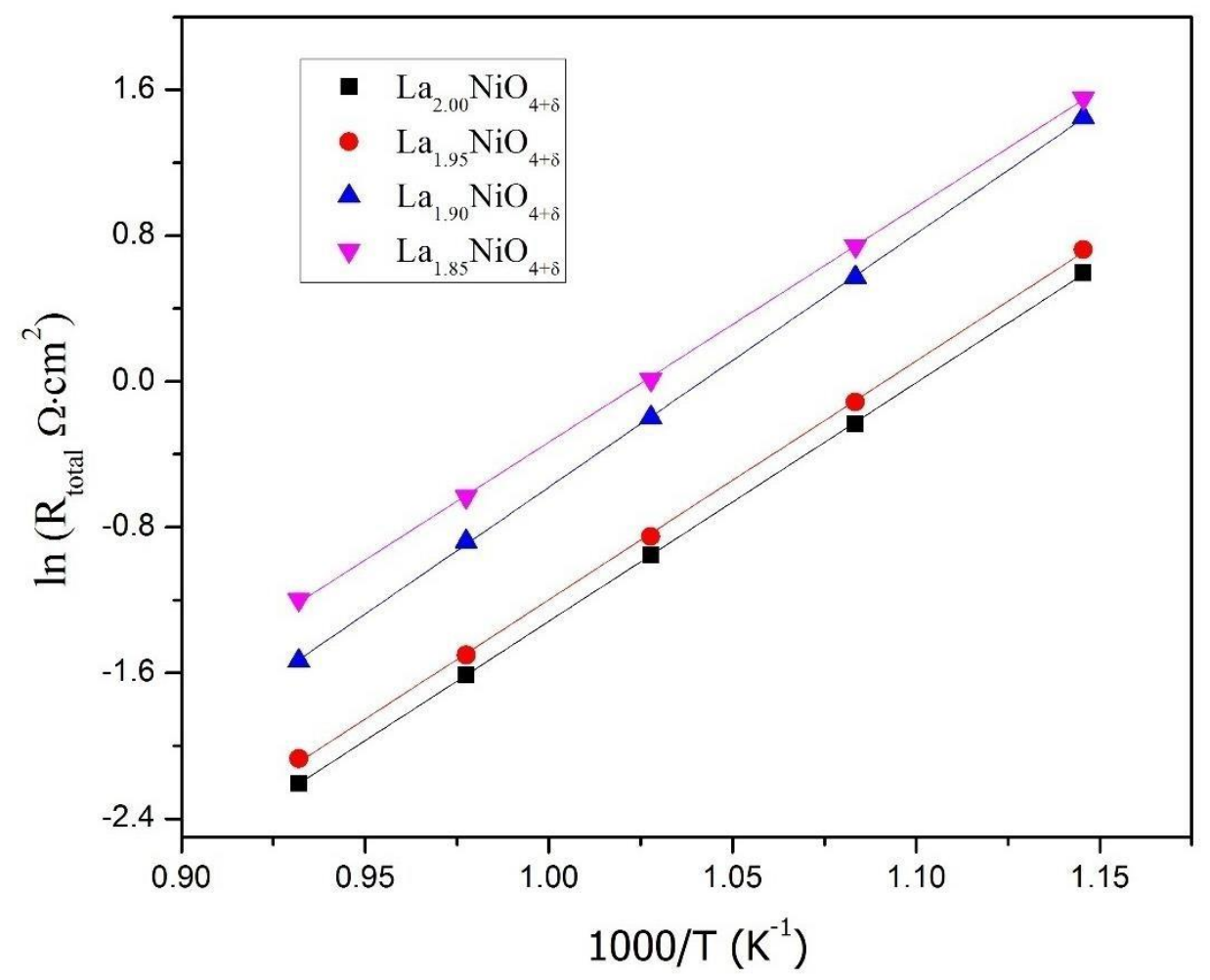

Figure 4.5 Temperature-dependent polarization cathode resistance of $\mathrm{La}_{2-\mathrm{x}} \mathrm{NiO}_{4+\delta}(\mathrm{x}=0-0.2)$ 


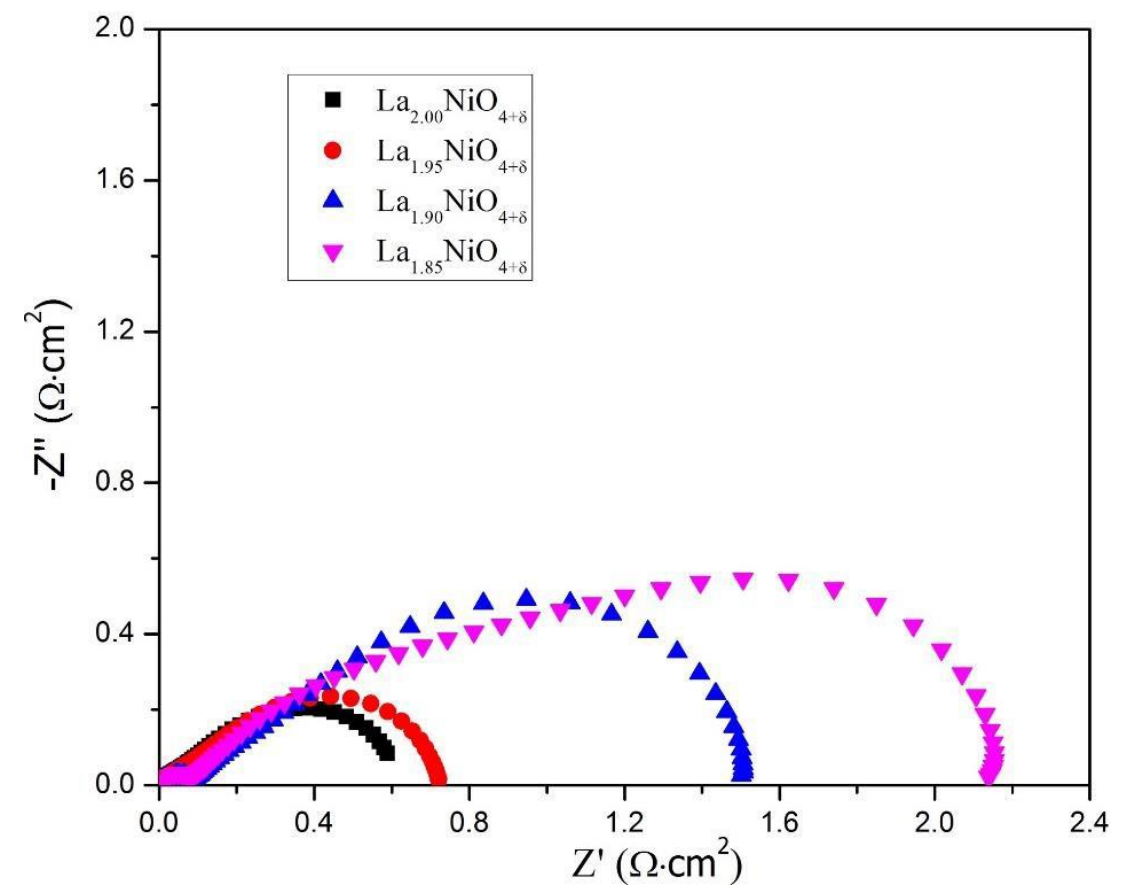

Figure 4.6 Temperature-dependent polarization cathode resistance of $\mathrm{La}_{2-\mathrm{x}} \mathrm{NiO}_{4+\delta}(\mathrm{x}=0-0.2)$ for high frequency arcs

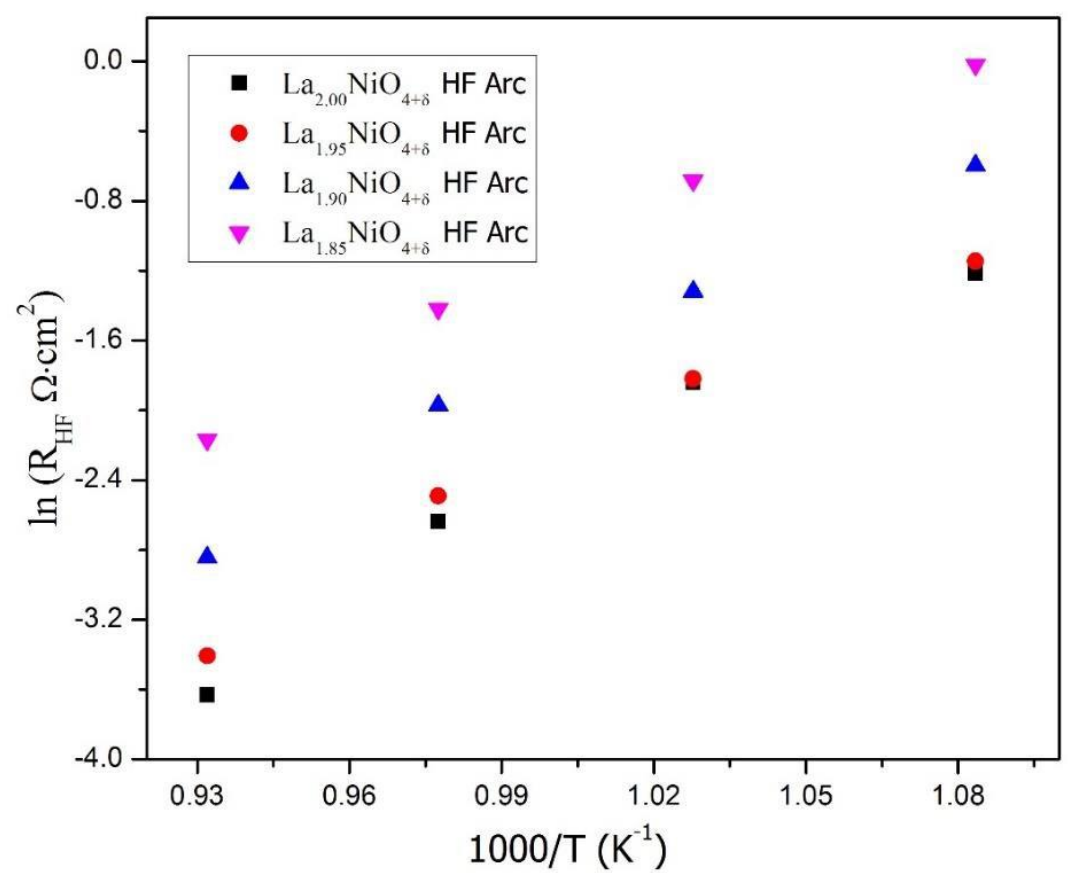

Figure 4.7 Temperature-dependent polarization cathode resistance of $\mathrm{La}_{2-\mathrm{x}} \mathrm{NiO}_{4+\delta}(\mathrm{x}=0-0.2)$ for high frequency arcs 


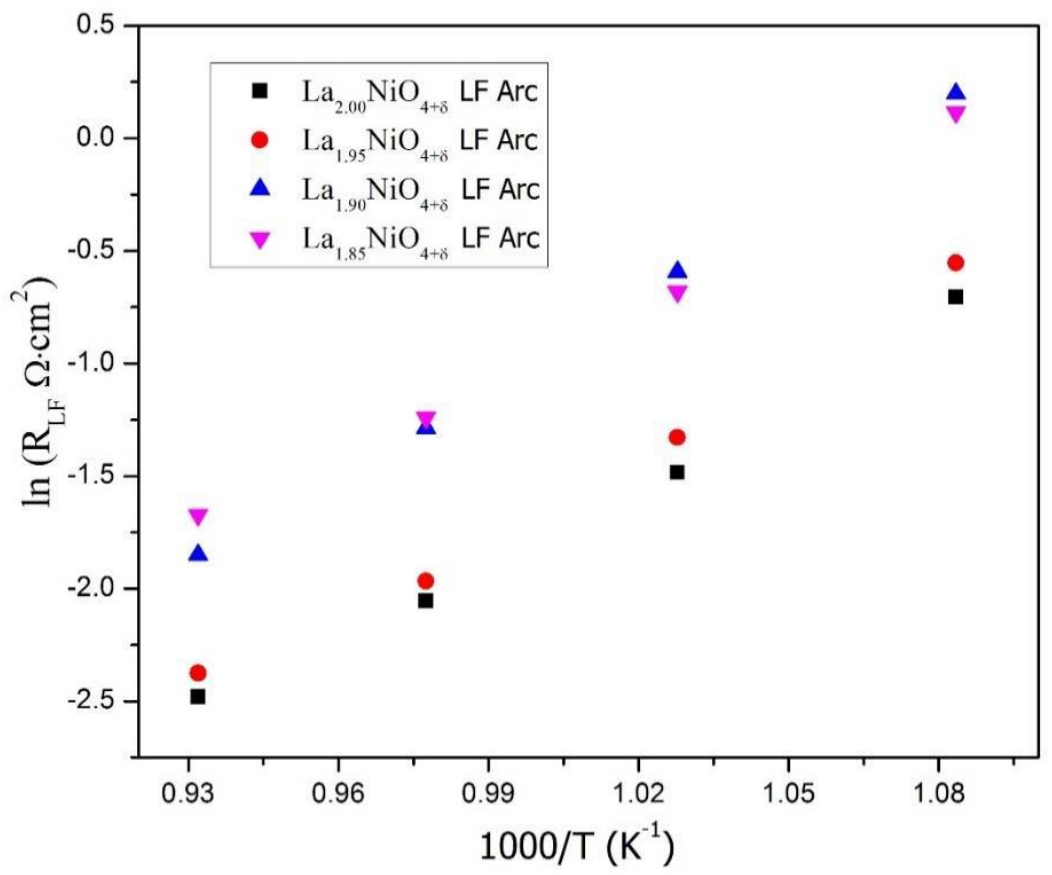

Figure 4.8 Temperature-dependent polarization cathode resistance of $\mathrm{La}_{2-\mathrm{x}} \mathrm{NiO}_{4+\delta}(\mathrm{x}=0-0.2)$ for low frequency arcs

\subsubsection{Infiltrated Hetero-structure A-site deficient LNO-LSCF Cathode}

For comparison and performance evaluation, $\mathrm{La}_{2-\mathrm{x}} \mathrm{NiO}_{4+\delta}(\mathrm{x}=0-0.2)$ was infiltrated into the both sides of LSCF layers in the LSCF|GDC|YSZ|GDC|LSCF cell to form a symmetric cell using LNO infiltrated LSCF cathode. Representative EIS data for symmetric cells using $\mathrm{La}_{2-\mathrm{x}} \mathrm{NiO}_{4+\delta}(\mathrm{x}=0-0.2)$ cathodes are collected at different temperature under OCV in air. Temperature-dependent polarization resistance is characterized in Fig. 4.9. The $R_{p}$ values of $\mathrm{La}_{2-\mathrm{x}} \mathrm{NiO}_{4+\delta}(\mathrm{x}=0-0.2)$ infiltrated LSCF cathodes are $0.025,0.028$ and $0.035 \Omega \cdot \mathrm{cm}^{2}$ at $750{ }^{\circ} \mathrm{C}$, respectively. The cathode electrochemical performance reduces with increasing the concentration of lanthanum deficient, which is consistent with the pure $\mathrm{La}_{2-\mathrm{x}} \mathrm{NiO}_{4+\delta}(\mathrm{x}=0-$ 0.2) cathode properties because of its surface electro-catalytic. The obvious one order of magnitude decrease in cathode polarization resistance after infiltration demonstrates that Asite deficient $\mathrm{La}_{2-x} \mathrm{NiO}_{4+\delta i}$ s still one potential infiltration materials for bare LSCF cathode. The cells with $\mathrm{La}_{2-x} \mathrm{NiO}_{4+\delta}$ infiltrated LSCF cathode will be assembled, and the stability of the cathode will be characterized in the future. 
To avoid the influence of microstructure variation of infiltrated nanoparticles, the stabilities of electrochemical performance of infiltrated LSCF were examined by dense $\mathrm{La}_{2-\mathrm{x}} \mathrm{NiO}_{4+\delta}$ coated LSCF thin cathode fabricated by air-brushed dry pressing method. The electrochemical impedance data were collected after different polarization times from 30960mins. In the all case of $\mathrm{La}_{2-x} \mathrm{NiO}_{4+\delta}(x=0-0.2)$ cathodes, two RQ elements of semi-circle arcs reflect the electrode polarization. The small high frequency arcs are correspond with electrode/electrolyte interface process based on the high frequency $10^{5} \mathrm{~Hz}$, and the large low frequency arcs reflect surface ORR process. The initial $\mathrm{R}_{\mathrm{p}}$ values of $\mathrm{La}_{2-\mathrm{x}} \mathrm{NiO}_{4+\delta}(\mathrm{x}=0-$ 0.2) coated LSCF cathodes increase with increasing the concentration of lanthanum deficient, which is consistent with the pure $\mathrm{La}_{2-\mathrm{x}} \mathrm{NiO}_{4+\delta}(\mathrm{x}=0-0.2)$ cathode properties because of its surface electro-catalytic. But after $-0.2 \mathrm{~V}$ cathodic polarization for $960 \mathrm{~min}$, the degradation of $\mathrm{La}_{2-\mathrm{x}} \mathrm{NiO}_{4+\delta}(\mathrm{x}=0-0.2)$ coated LSCF reduces with increasing lanthanum deficient concentration. The enhanced stability of $\mathrm{La}_{2-\mathrm{x}} \mathrm{NiO}_{4+\delta}(\mathrm{x}=0-0.2)$ coated LSCF demonstrates that A-site deficient $\mathrm{La}_{2-x} \mathrm{NiO}_{4+\delta}$ is one potential infiltration materials for bare LSCF cathode to improve the surface stability. The La/Ni ratio determined by surface XPS also proved that A-site cation segregation can be suppressed by the introduction of A-site deficiency slightly. 


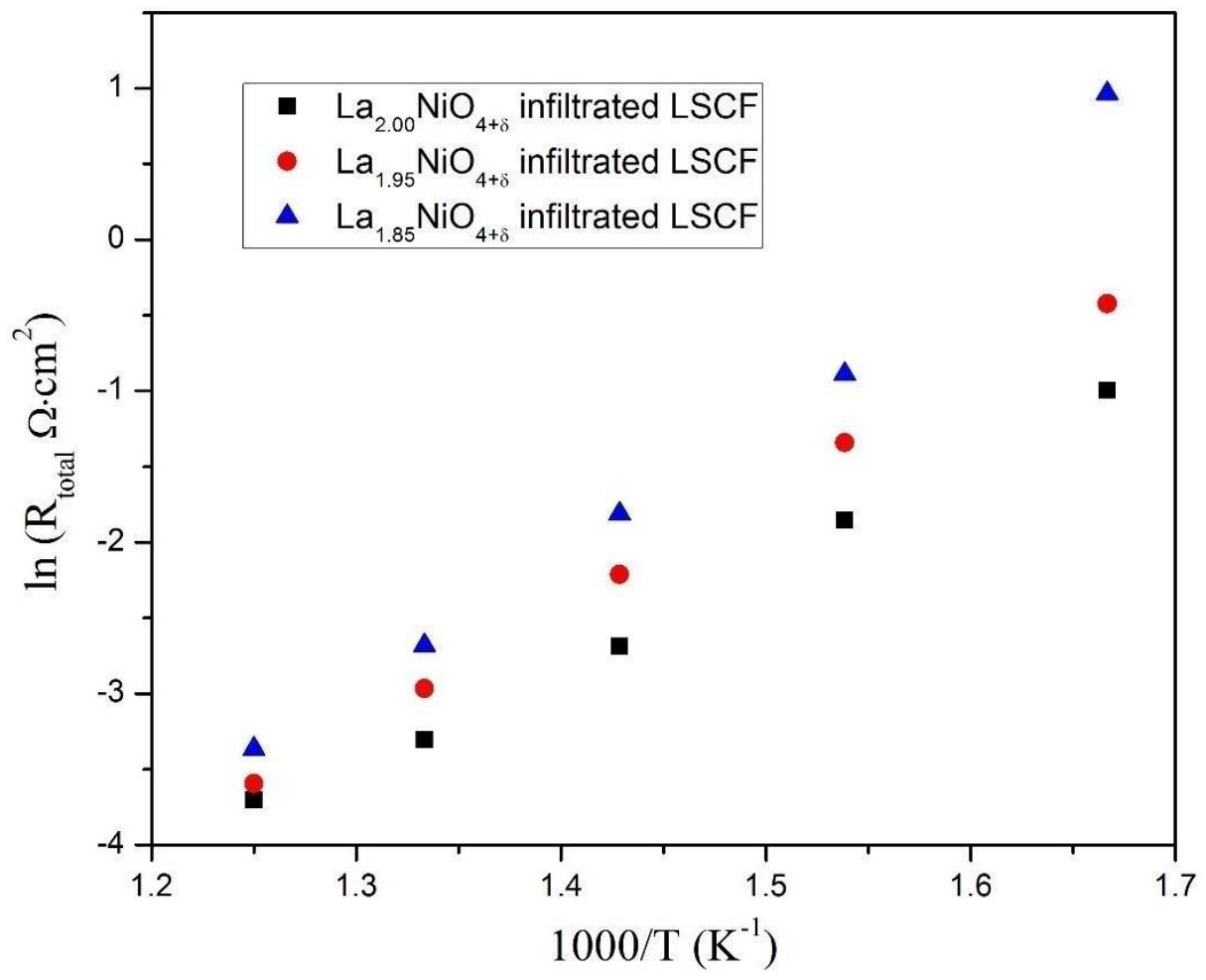

Figure 4.9 Temperature-dependent polarization cathode resistance of $\mathrm{La}_{2-\mathrm{x}} \mathrm{NiO}_{4+\delta}(\mathrm{x}=0-0.2)$ infiltrated LSCF
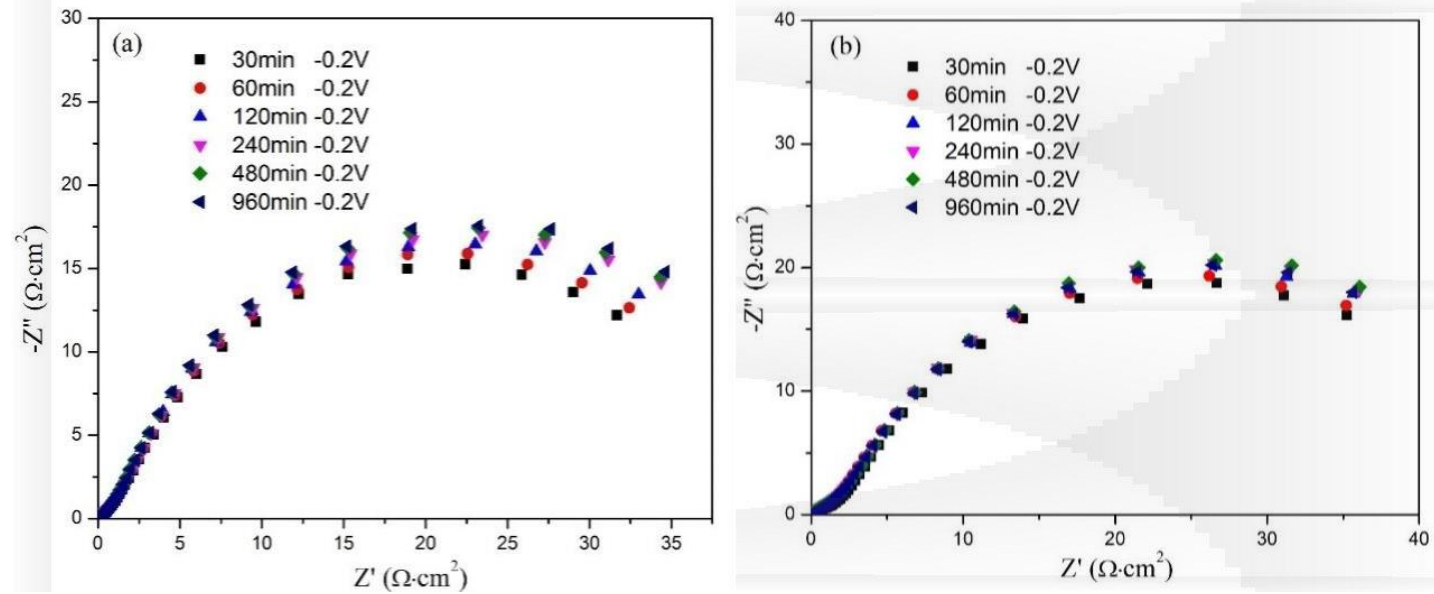


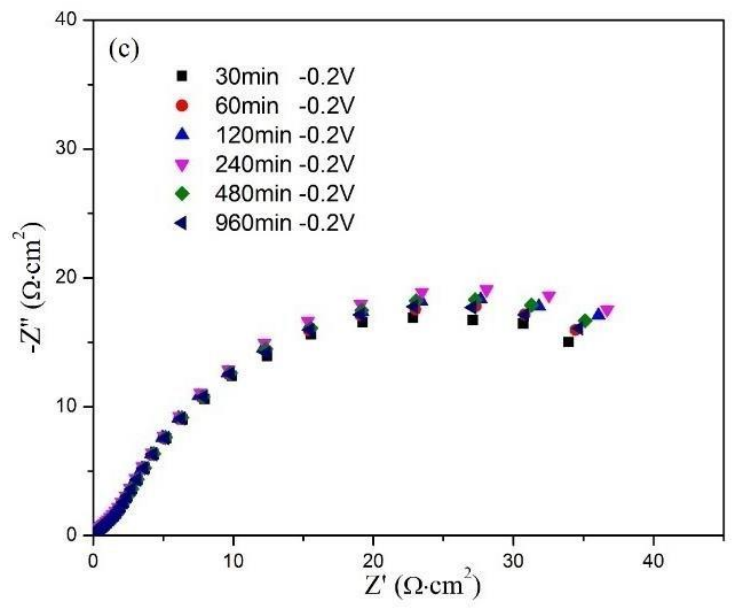

Figure 4.10 Nyquist plot of three electrode cell with A-site deficient $\mathrm{La}_{2-x} \mathrm{NiO}_{4+\delta}$ coated $\mathrm{LSCF}$ cathode at $700^{\circ} \mathrm{C}$ after $-0.2 \mathrm{~V}$ cathodic polarization (a) $\mathrm{La}_{2} \mathrm{NiO}_{4+\delta}$, (b) $\mathrm{La}_{1.95} \mathrm{NiO}_{4+\delta}$ and (c) $\mathrm{La}_{1.85} \mathrm{NiO}_{4+\delta}$

Table 4.4 The ratio of $\mathrm{La} / \mathrm{Ni}$ in $\mathrm{La}_{2-x} \mathrm{NiO}_{4+\delta}$ with and without long-term operation

\begin{tabular}{|c|c|c|c|c|}
\hline & $\mathrm{La}_{2} \mathrm{NiO}_{4+\delta}$ & $\mathrm{La}_{1.95} \mathrm{NiO}_{4+\delta}$ & $\mathrm{La}_{1.90} \mathrm{NiO}_{4+\delta}$ & $\mathrm{La}_{1.85} \mathrm{NiO}_{4+\delta}$ \\
\hline Initial $\mathrm{La}^{3+} / \mathrm{Ni}^{2+}$ ratio & 2.29 & 2.10 & 1.94 & 1.83 \\
\hline $\mathrm{La}^{3+} / \mathrm{Ni}^{2+}$ ratio after long-term operation & 3.94 & 3.43 & 3.14 & 2.61 \\
\hline
\end{tabular}

\subsection{Summary}

A-site deficient LNO were prepared to inhibit the lanthanum segregation on the LNOLSCF surface, since some lanthanum oxide and lanthanum nickel perovskite phase were detected after polarization treatment on LNO/LSCF surface in previous work. The intrinsic properties of $\mathrm{La}_{2-\mathrm{x}} \mathrm{NiO}_{4+\delta}(\mathrm{x}=0-0.2)$ were measured. The maximum deficient concentration of lanthanum is less than 0.2 in $\mathrm{La}_{2-x} \mathrm{NiO}_{4+\delta}$. The $\mathrm{La}_{2-x} \mathrm{NiO}_{4+\delta}$ with higher deficient exhibits a slightly higher conductivity below $800^{\circ} \mathrm{C}$. Part of lanthanum deficient was compensated by holes and increase the concentration of $\mathrm{Ni}^{3+}-\mathrm{O}-\mathrm{Ni}^{2+}$ transport way. The concentration of interstitial oxygen defects and the paths of interstitial oxygen transport decrease with the addition of A-site deficiency. Although the $\mathrm{La}_{2-\mathrm{x}} \mathrm{NiO}_{4+\delta}$ with higher deficiency has higher conductivity, the surface charge transfer still dominates the impedance with increasing lanthanum deficiency. The surface charge transfer process for LNO materials is possibly 
controlled by surface interstitial oxygen concentration along with transition metal states. A one order of magnitude decrease in cathode polarization resistance after infiltration has been observed, and after $-0.2 \mathrm{~V}$ cathodic polarization for $960 \mathrm{~min}$, the degradation of $\mathrm{La}_{2}$ ${ }_{x} \mathrm{NiO}_{4+\delta}(\mathrm{x}=0-0.2)$ coated LSCF reduces with increasing lanthanum deficient concentration. The enhanced stability of $\mathrm{La}_{2} \mathrm{x} \mathrm{NiO}_{4+\delta}(\mathrm{x}=0-0.2)$ coated LSCF demonstrates that A-site deficient $\mathrm{La}_{2-\mathrm{x}} \mathrm{NiO}_{4+\delta}$ is one potential infiltration materials for bare LSCF cathode to improve the surface stability. But more investigation needs to be done to further solve the problem of lanthanum enrichment. 


\section{Chapter 5: $\mathrm{CeO}_{2} \& \mathrm{La}_{2} \mathrm{NiO}_{4+\delta} \mathrm{Co}$-Infiltrated $\left(\mathrm{La}_{0.6} \mathrm{Sr}_{0.4}\right)_{0.95} \mathrm{Co}_{0.2} \mathrm{Fe}_{0.8} \mathrm{O}_{3-\delta}$ Cathode}

\subsection{Introduction}

Reducing the operation temperature range of solid oxide fuel cells (SOFCs) from 800$1000^{\circ} \mathrm{C}$ to $600-800^{\circ} \mathrm{C}$ is currently attracting the attention of researchers, because it is beneficial to the long-term stability, the flexibility of material choices and thus the reduction of the cost of SOFCs systems $\left[{ }^{9,10,229}\right]$. As the performance of the typical hightemperature strontium-doped lanthanum manganite (LSM) electrode is not satisfactory in this intermediate-temperature region, new composite cathode materials with rapid oxygen exchange and transport properties, such as LSCF and LNO, have been developed to promote the electrode performance. Besides the exploration of novel materials, another efficient approach to improve the performance is infiltration. The infiltration technique has been extensively utilized in the composite cathode fabrication based on its unique advantages among other composite techniques. As results of the low phase formation temperature and nano-sized deposit particles, the chemical reaction between the infiltrated materials and the electrolyte can be diminished, and large triple-phase boundary (TPB) areas and high catalytic activity can be acquired for cathode reaction[ $\left.{ }^{191-193}\right]$. Moreover, infiltration may benefit the performance by avoiding an inert surface. In our previous study, it has been demonstrated that an LNO infiltration layer can suppress the surface segregation of $\mathrm{Sr}$ on the LSCF backbone $\left.{ }^{230}\right]$. In fact, numerous cathode infiltration investigations indicate that the electrode polarization resistance of common electrolyte infiltrated LSM was one or two orders of magnitude smaller than the value of the pure LSM under intermediate temperature. Nonetheless during the long-term operation, the infiltrated cathode usually experiences an abrupt performance drop in the early stage, which compromises the advantage of such method in the commercial application. The agglomeration/growth and delamination of the infiltrant particles due to low melt point and/or high surface energy are believed to be the main reasons for the degradation of the electrode performance under SOFCs operation temperature $\left.{ }^{205,207,231-234}\right]$.

It is well-known that melting-point depression phenomenon plays a critical role in the growth of nano-sized particles. The melting point of materials reduces with decreasing 
particle size. For the particle growth process, three major mechanisms have been developed and applied in the investigations of the initial stage of solid sintering according to the characteristic of mass transfer, solid state atomic diffusion, liquid phase transport and evaporation condensation $\left[{ }^{235-238}\right]$. Vapor involved evaporation condensation process is common to be observed in metal particles growth, but not in ceramics particles considering the high evaporation temperature for ceramics. While, solid and liquid related mass transfer processes are widely applied in ceramics growth and sintering, as shown in Fig. 5.1. For the solid-state mass transfer, atomic diffusion in the solid state produces joining of the particles and enhancement of particle growth, and usually the particles growth and aggregation happen when the temperature reaches to typically $0.5-0.9$ of the melting point $\left[{ }^{237}\right]$. For the liquid phase mass transfer, a small amount of liquid or glass phases flow from particles surface to the grain boundary and lead to boundary growth of the particles. The present of liquid or glass phase is controlled by the melting point or the softening temperature of particles. Both atomic diffusion mechanism and liquid viscous flow mechanisms, are mostly related with the melting point of particles. .

Consequently, the nanoparticle growth and aggregation take place in the infiltrated cathode during operation even though the temperature is still much lower than the melting point. Therefore, suppression of melting-point depression is key factor to inhibit the nanoparticles aggregation/growth and enhance the stability of infiltration electrode. In this sense, a possible way to depress the nanoparticles growth and aggregation is to add a second phase with higher melting point and poor atomic diffusion property. The common cubic fluorite structure electrolytes used in SOFCs, such as doped $\mathrm{ZrO}_{2}$ or $\mathrm{CeO}_{2}$-based materials, show a up to $500^{\circ} \mathrm{C}$ higher melting point than the common cathode materials $\left[{ }^{239}\right]$. Compared with doped $\mathrm{ZrO}_{2}$-based materials, $\mathrm{CeO}_{2}$-based electrolytes display lower inter-diffusion tendency with cathode elements, and thereby have been widely used as an interlayer between YSZ and cathode to restrict the diffusion of lanthanum and strontium etc. [ $\left.{ }^{240,241}\right]$. Meanwhile, it has been proved that the wettability of $\mathrm{CeO}_{2}$-based materials is much greater towards LSCF and LSC cathode materials. Therefore, a resultant good adhesion with cathode backbone would probably prevent the delamination of infiltration layer $\left[{ }^{242}\right]$. In regard of this, $\mathrm{CeO}_{2}$ could be a desirable second phase to reduce nanoparticles growth and 
enhance the long term stability of infiltrated electrode. The high conductivity and oxygen defect concentration $\mathrm{CeO}_{2}$-based materials are desired to mitigate the problem of the conductivity shortage of infiltration phases and improve the effective oxygen kinetics in $\mathrm{CeO}_{2} / \mathrm{LNO} /$ gas triple phase boundary. Researchers have reported that the substitution of lanthanide series elements in $\mathrm{CeO}_{2}$ enhance the defect concentration and the total conductivity. The total conductivities of (Gd, Sm, La)-doped $\mathrm{Ce}_{1-\mathrm{x}} \mathrm{MO}_{2}(\mathrm{M}=\mathrm{Gd}, \mathrm{Sm}, \mathrm{La})$ are two orders of magnitude higher than pure $\mathrm{CeO}_{2}\left[{ }^{243},{ }^{244}\right]$. In the consideration of the coexistence of lanthanum and cerium nitrates in the infiltration solution, it is assumed that La-doped $\mathrm{CeO}_{2}$ (LDC) could be formed in one-step co-infiltration process. Therefore, no Gd or Sm was added into infiltration solution to avoid complexity of infiltration solution. In the present study, in continuation of our previous study on LNO-infiltrated $\mathrm{LSCF}, \mathrm{CeO}_{2}$ was co-infiltrated into the LSCF backbone along with $\mathrm{La}_{2} \mathrm{NiO}_{4+\delta}$ to prevent the particle growth/ aggregation and delamination from LSCF surface. The morphology and electrochemical performance of co-infiltration cathodes were monitored to reveal the effect of $\mathrm{CeO}_{2}$ on the long term aging process.

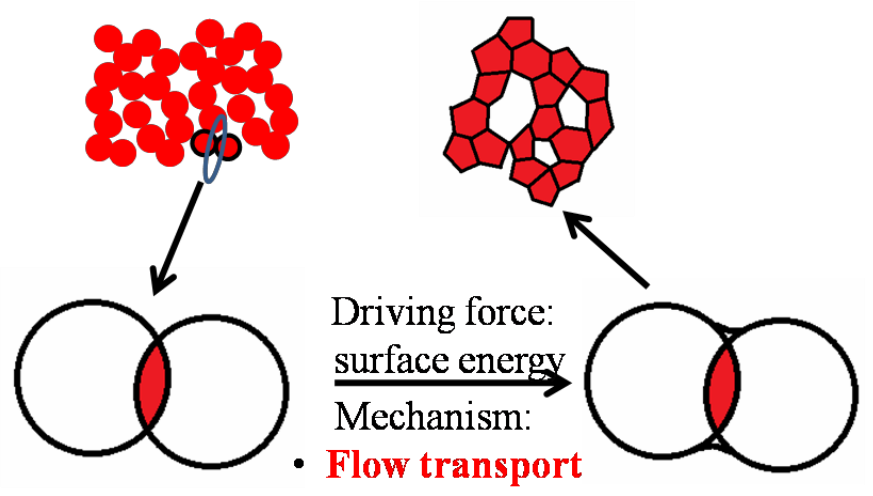

Figure 5.1 Schemic of the aggregation and sintering process of particles

\subsection{Experimental}

A symmetric cell configuration was adopted. Gadolinium doped ceria (10\% Gd, GDC, Fuel Cell Material Co.) powders were dry-pressed under 300MPa in a $16.5 \mathrm{~mm}$ die, followed by sintering at $1450^{\circ} \mathrm{C}$ for $4 \mathrm{~h}$ to fabricate dense electrolyte pellets. The thickness of these pellets is $\sim 1 \mathrm{~mm}$. A-site deficiency $\left(\mathrm{La}_{0.6} \mathrm{Sr}_{0.4}\right)_{0.95} \mathrm{Co}_{0.2} \mathrm{Fe}_{0.8} \mathrm{O}_{3-\delta}$ (LSCF, Fuel Cell Material 
Co.) powders were dispersed into an organic vehicle ink (VHE, Fuel Cell Material Co. in US.) with a weight ratio of 1:0.7 to form a homogeneous slurry. Then, the slurry was screen-printed on both sides of the GDC electrolyte symmetrically, and sintered at $1150^{\circ} \mathrm{C}$ for $3 \mathrm{~h}$. The LSCF cathodes have a thickness of $20 \mu \mathrm{m}$ and an active surface area of 0.7 $\mathrm{cm}^{2}$. $\mathrm{CeO}_{2}$ and LNO were infiltrated into the both sides of the LSCF|GDC|LSCF cell with different molar ratio, as described below, to form $\mathrm{CeO}_{2} \& \mathrm{LNO}$ co-infiltrated LSCF cathodes.

The $\mathrm{CeO}_{2} \& \mathrm{LNO}$ precursor solution with a concentration of $1 \mathrm{~mol} / \mathrm{L}$ was composed of $\mathrm{La}\left(\mathrm{NO}_{3}\right)_{3} \cdot 6 \mathrm{H}_{2} \mathrm{O}, \mathrm{Ni}\left(\mathrm{NO}_{3}\right)_{2} \cdot 6 \mathrm{H}_{2} \mathrm{O}$ and $\mathrm{Ce}\left(\mathrm{NO}_{3}\right)_{2} \cdot 6 \mathrm{H}_{2} \mathrm{O}(99.9 \%$, Alfa Aeser) as solute, a mixture of ethanol and deionized water with a volume ratio of 1:0.6 as solvent. Citric acid and TX100 was added into the solution as complexing agent and dispersant to promote the formation of $\mathrm{LNO}$ phase and improve $\mathrm{CeO}_{2}$ phase distribution status on the LSCF substrate. After infiltrating the nitrate solution into the porous LSCF cathode on each side of the symmetric cells, the samples were dried at $250^{\circ} \mathrm{C}$ for $1 \mathrm{~h}$. Repeating infiltration process followed by drying process was carried out to increase the amount of infiltration phase in the cathode backbone. Eventually the cells were sintered at $900^{\circ} \mathrm{C}$ for $1 \mathrm{~h}$ to crystallize the $\mathrm{CeO}_{2}$ and LNO phases. Afterward, silver ring was attached to the electrodes of the symmetric cells as current collector by silver paste and sintered at $700 \mathrm{oC}$ for half an hour.

To confirm the existence of $\mathrm{CeO}_{2}$ and $\mathrm{LNO}$ phases and the compatibility between $\mathrm{CeO}_{2}$ and $\mathrm{LNO}, \mathrm{CeO}_{2}$ and $\mathrm{LNO}$ particles were co-fabricated on flat LSCF plate to avoid the inconvenient XRD detection for porous electrode with similar infiltration solution. The resultant basic LSCF plate were sintered in air at $900^{\circ} \mathrm{C}$ for $1 \mathrm{~h}$. X-ray diffraction (XRD) patterns were gathered by a Panalytical X'Pert Pro diffractometer using $\mathrm{Cu} \mathrm{K} \alpha$ radiation $(\lambda=1.5406 \AA)$ to identify the phase composition. The cross section microstructure of infiltrated cathode with or without ageing at $750^{\circ} \mathrm{C}$ for $1080 \mathrm{~h}$ were recorded by scanning electron microscope (SEM, JEOL JSM-7600F). A Metrohm Autolab station (AUT85484) was applied on the symmetrical cells for electrochemical impedance spectroscopy (EIS) measurement under open circuit voltage (OCV) in ambient air from $550^{\circ} \mathrm{C}$ to $750^{\circ} \mathrm{C}$. The frequency range was from 0.01 to $100 \mathrm{kHz}$ with a $10 \mathrm{mV}$ ac amplitude. All impedance data were collected after a stabilization time of more than $30 \mathrm{~min}$ and analyzed using Z-view 3.1. 


\subsection{Results and Discussion}

\subsubsection{X-ray Diffraction Pattern for phase composition}

Fig. 5.2 shows the XRD pattern of the $\mathrm{CeO}_{2} \&$ LNO co-infiltrated LSCF electrode on GDC electrolyte. Although the main characteristic peaks of LNO can be identified in the XRD patterns, the characteristic peaks of $\mathrm{CeO}_{2}$ will be eclipsed by the GDC electrolyte peaks. The intensity of the LNO peaks were found much weak than the LSCF backbone and some characteristic peaks may merge with the backbone peaks also. In short, the crystal structure of the in-situ formed $\mathrm{LNO}$ and $\mathrm{CeO}_{2}$ cannot be clearly verified through the pellet XRD. Therefore, to further confirm the existence of $\mathrm{CeO}_{2}$ and $\mathrm{LNO}$ phases, the metal nitrate infiltration solutions of $\mathrm{CeO}_{2} \& \mathrm{LNO}$ were also dipped on flat LSCF plate. After drying at $250^{\circ} \mathrm{C}$, the precursor powders was co-sintered at $900^{\circ} \mathrm{C}$ for $1 \mathrm{~h}$ to obtain final product. XRD pattern of the co-synthesized powders was collected, as shown in Fig. 5.3. Only LNO peaks with tetragonal structure and $\mathrm{CeO}_{2}$ peaks were seen in the patterns. But the position of $\mathrm{CeO}_{2}$ peaks move to the smaller angle, which means larger lanthanum ion was possibly doped into $\mathrm{CeO}_{2}$ lattice when mixing cerium and lanthanum ions together. Trivalent lanthanum doping in $\mathrm{CeO}_{2}$ improves the total conductivity of pure ceria by two orders of magnitude due to the generation of intrinsic oxygen vacancies $\left[{ }^{244}\right]$. The enhancement of the total conductivity could supply more oxygen transport paths and improve the concentration of oxygen incorporation sites in the cathode. And valence alternation of cerium could offer a high catalytic activity for oxygen reduction reaction. Meanwhile, the doping of $\mathrm{La}$ in $\mathrm{CeO}_{2}$ results in A-site deficient in $\mathrm{LNO}$ phase since the stoichiometric lanthanum nitrite and nickel nitrite were mixed in infiltration solution preparation. It could enhance the total conductivity of LNO that is the main disadvantage for LNO-based materials, and may suppress the surface absence of Ni problems 


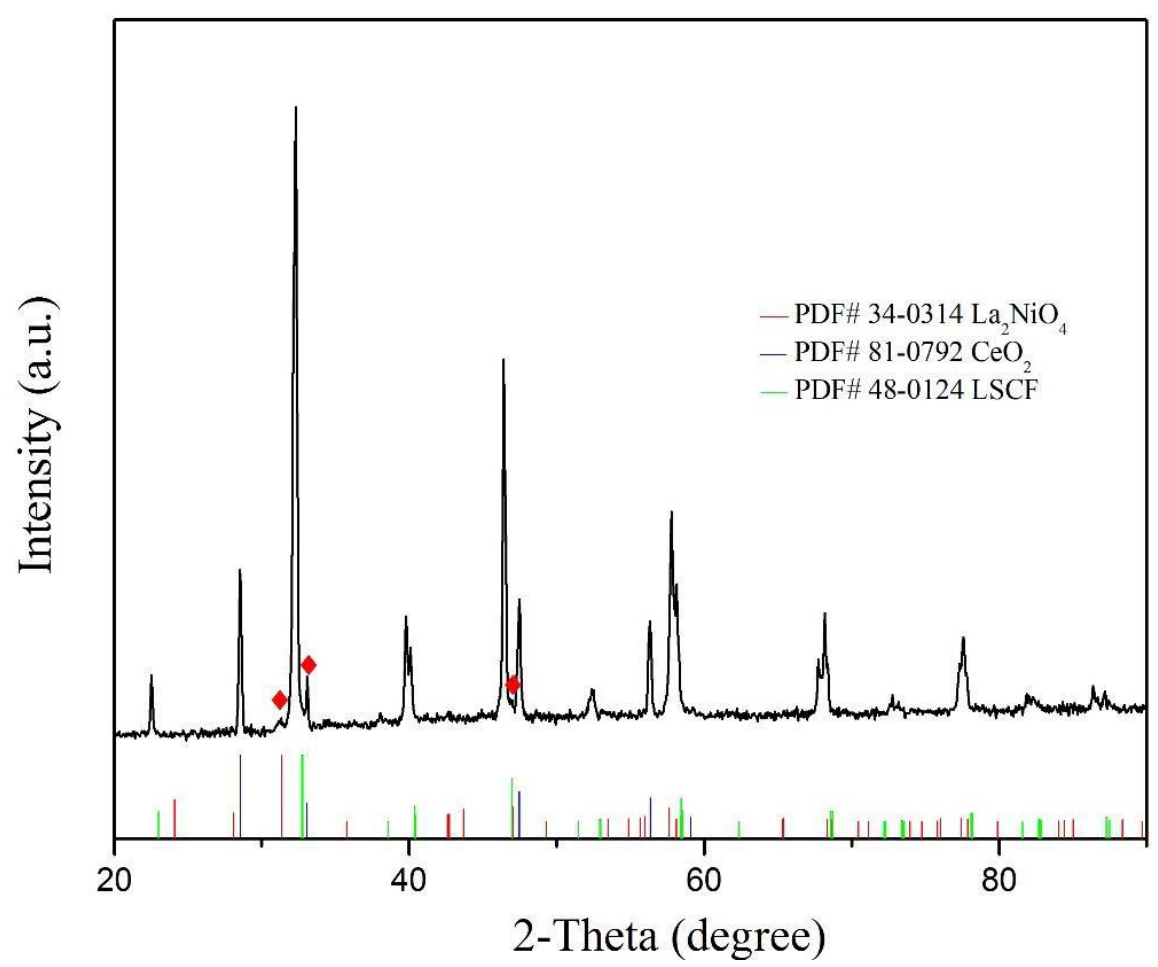

Figure 5.2 XRD pattern of $\mathrm{CeO}_{2} \& \mathrm{LNO}$ co-infiltrated LSCF/GDC/LSCF cells

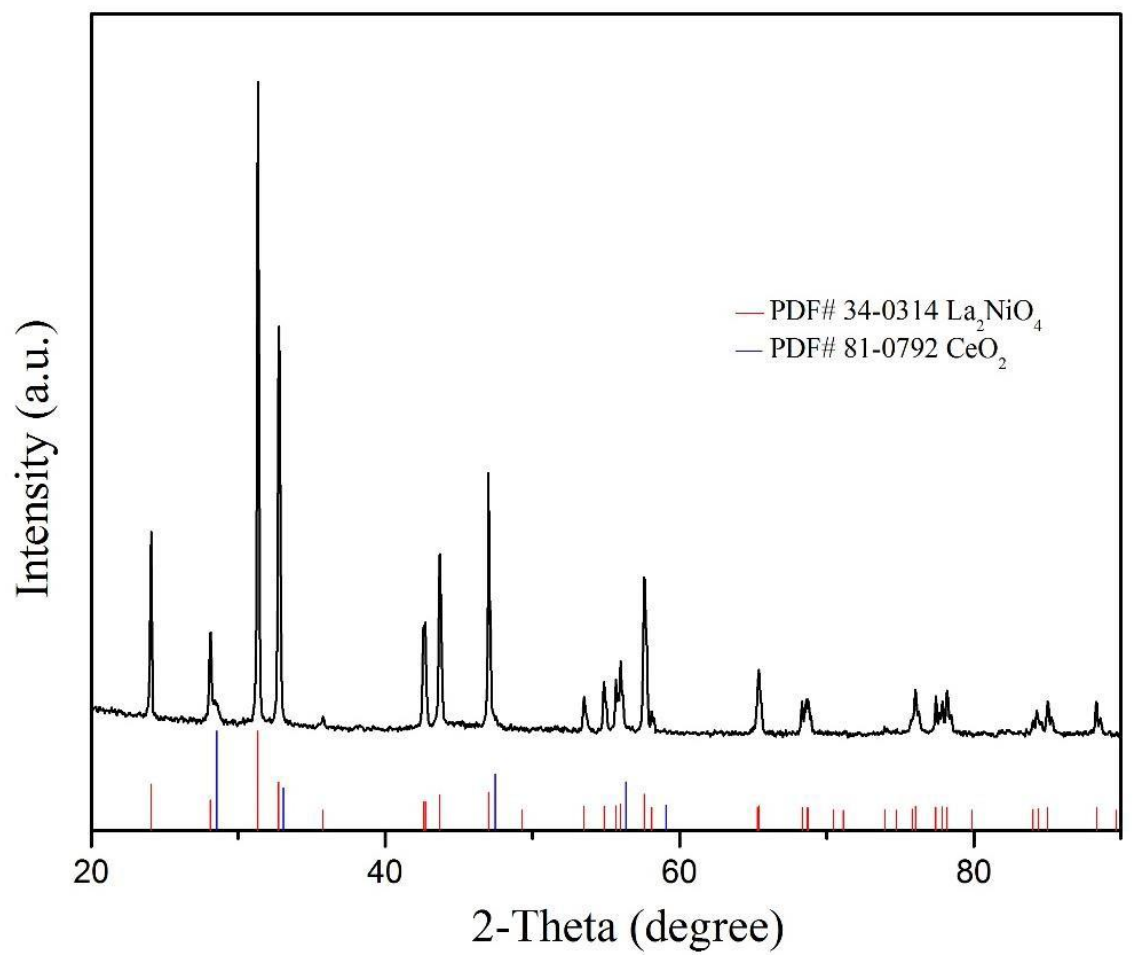

Figure 5.3 XRD pattern of $\mathrm{CeO}_{2} \& \mathrm{LNO}$ powders co-synthesized by sol-gel solution 
5.3.2 Effect of microstructure before and after long-term stability

Fig.5.4 (a)-(d) show the SEM microstructures of the as-prepared pure LNO-infiltrated LSCF and the as-prepared $20 \mathrm{~mol}$. $\% \mathrm{CeO}_{2} \& \mathrm{LNO}$ co-infiltrated LSCF. For both asprepared cathodes, the nanoparticles are uniformly distribute on the surface of the LSCF backbone and closely connect to the LSCF backbone. In LNO-only sample, the clear particles boundary can be seen between the LNO nanoparticles with a diameter about 50$100 \mathrm{~nm}$ in the magnified SEM figure. And comparing with the pure LNO infiltration, the addition of $\mathrm{CeO}_{2}$ increases the uniformity and wettability of infiltration solutions and reduces the particles size slightly, thereby enlarging the surface and nanoparticles/backbone interface areas with a flattened morphology.

However, Fig. 5.4 (e)- (h) the microstructures of LNO-only infiltrated LSCF and $\mathrm{CeO}_{2}$ \& LNO co-infiltrated LSCF after $1080 \mathrm{~h}$ at $750^{\circ} \mathrm{C}$ treatment. It can be seen that the LNO particles aggregate with each other and form a continuous network of solid nanoparticles and porosity with ambiguous grain boundary. The diameter of some isolated grown particles reach to $200-300 \mathrm{~nm}$, and much larger size of the compact particles with ambiguous boundary can be detected in the Fig. 5.4 (e) and (f). It looks like the typical intermediate stage of particles sintering. In that stage, the high curvatures of the initial state have been moderated and the microstructure consists of a three-dimensional interpenetrating network of solid particles and continuous, channel-like pores. And grain growth of the compact particles starts to become significant by grain boundary integration in the next process. Such aggregation and the following grain growth could reduce the surface areas of LNO particles, and then will probably impair the performance of the electrode. Besides, these LNO nanoparticles are more loosely adhered on the LSCF backbone in the LNO-only sample as shown in Fig. 4 (c). And even some nanoparticles delaminated from the LSCF surface and formed their own networks. Both phenomena reduce the effective $\mathrm{LNO} / \mathrm{LSCF}$ interface that is in favor of the enhancement of the electrochemical performance $\left[{ }^{162,245}\right]$. In contrast, for $\mathrm{CeO}_{2} \&$ LNO co-infiltrated LSCF samples, Fig. 5.4 (g) and (h) show an inhomogeneous size distribution of the nanoparticles with clear grain boundary. A part of nanoparticles kept the similar particle size with a diameter of 50-100nm after $1080 \mathrm{~h}$ aging, and a few nanoparticles surrounded by small 
particles grows to large particles with a diameter of less than $200 \mathrm{~nm}$. It seems that the second phase has the pinning effect on the movement of the grain boundary, resulting in the reduction of the grain boundary energy and the capacity of grain boundary movement. The particle size reaches steady state with a diameter of less than $200 \mathrm{~nm}$ and the particles growth process is completed. Hence, the existence of the second phase $\mathrm{CeO}_{2}$ nanoparticles suppress the atomic diffusion between LNO particles and further inhibits the nanoparticles growth/aggregation. It is benefit for the control of the reduction of surface $2 \mathrm{~PB}$ and $3 \mathrm{~PB}$ area after long-term operation. Meanwhile, the co-infiltrated nanoparticles are still tightly attached to the backbone surface uniformly. It avoids the exposition of LSCF backbone and restrains possible segregation of strontium on LSCF surface during long-term operation.
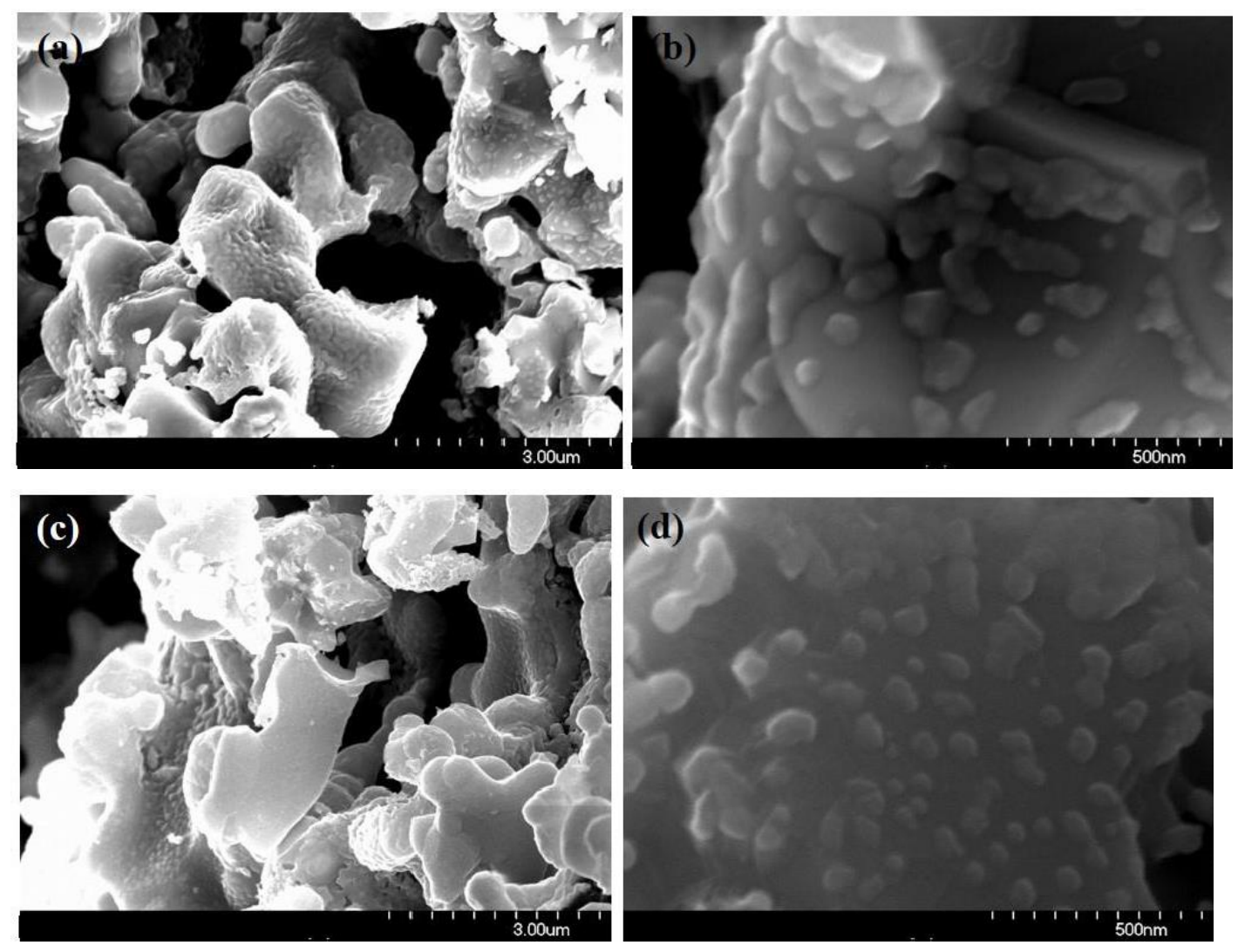


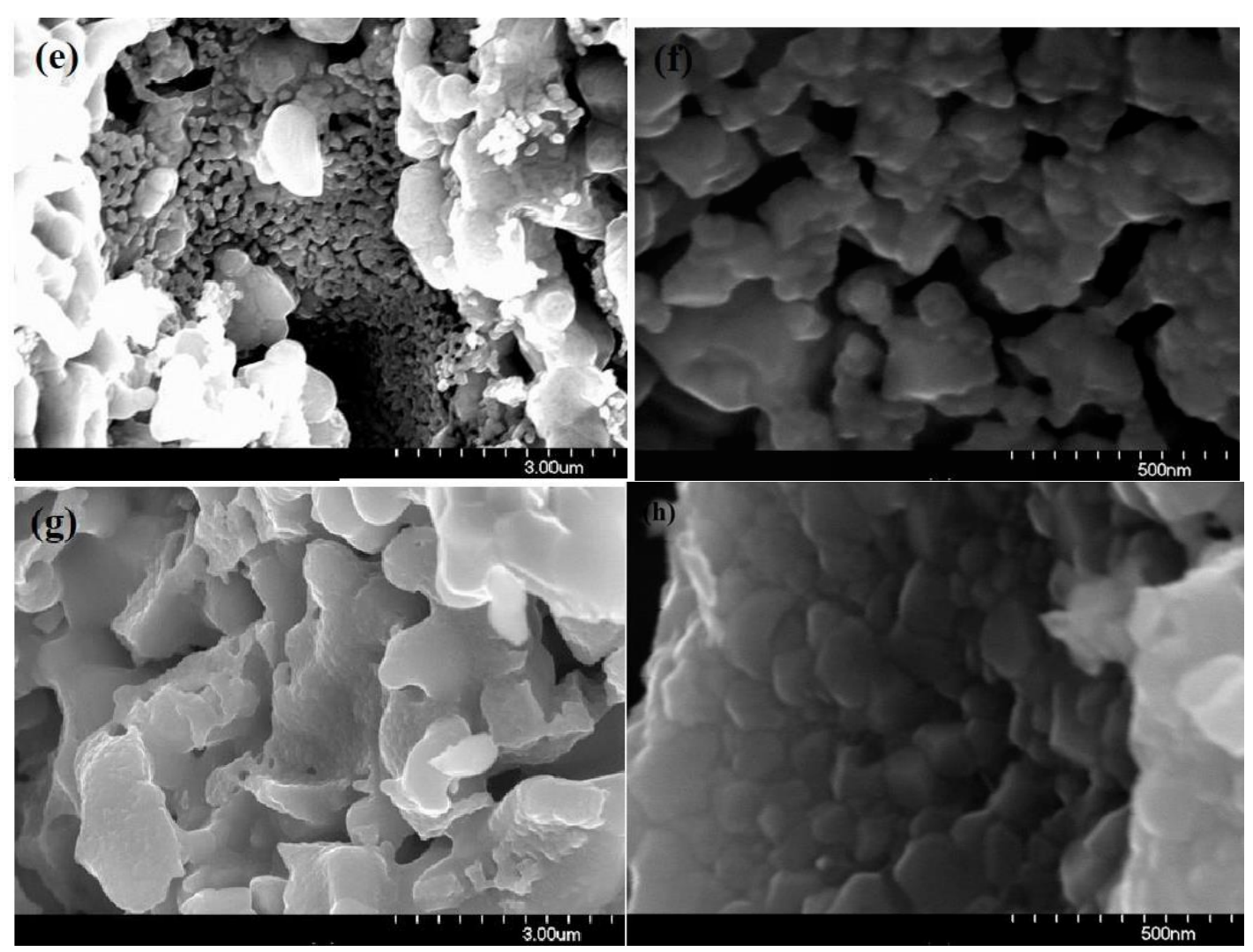

Figure 5.4 SEM image of (a) and (b) as-prepared LNO-infiltrated LSCF electrode, (c) and (d) LNO-infiltrated LSCF electrode with $1080 \mathrm{~h}$ heat treatment at $750^{\circ} \mathrm{C}$, (e) and (f) asprepared 20 mol. \% $\mathrm{CeO}_{2} \& \mathrm{LNO}$ co-infiltrated electrode, (g) and (h) $20 \mathrm{~mol}$ \% $\mathrm{CeO}_{2}$ \& LNO co-infiltrated LSCF electrode with $1080 \mathrm{~h}$ heat treatment at $750^{\circ} \mathrm{C}$

\subsubsection{Electrochemical Performance}

Firstly, in order to confirm the optimal loading of infiltration phase, the electrochemical performance of LNO-LSCF/GDC/LNO-LSCF symmetrical cells with different LNO loading were characterized in our preliminary work. With increasing the loading of LNO, the distribution of LNO nanoparticles in the LSCF substrate are of island, film or multiple sheet. Therefore, 15 wt. \% LNO loading, which covers on LSCF surface completely with a single uniform LNO nano-layer, was applied in our next work.

The electrochemical performances for these infiltrated LSCF cathodes were characterized by electrochemical impedance spectroscopy. The Arrhenius plots of the polarization resistance $\left(R_{\mathrm{p}}\right)$ are shown in Fig. 5.5 for samples with different $\mathrm{CeO}_{2}$ weight ratio. It 
indicates that $R_{\mathrm{p}}$ increases with increasing amount of $\mathrm{CeO}_{2}$ in the infiltration solution. The Rp values for as-prepared LNO-infiltrated LSCF cathode is $0.02 \Omega \mathrm{cm}^{2}$ at $750^{\circ} \mathrm{C}$. In comparison, Rp for the as-prepared co-infiltrated LSCF cathode is 0.05 and $0.12 \Omega \mathrm{cm}^{2}$ for $10 \mathrm{~mol} . \%$ and $20 \mathrm{~mol} . \% \mathrm{CeO}_{2}$ at $750^{\circ} \mathrm{C}$, respectively. Although, $\mathrm{CeO}_{2}$-containing electrode shows inferior performance than the LNO-only sample, their Rp are still one order of magnitude smaller than the blank LSCF cathode with less than $35 \mathrm{~mol} . \% \mathrm{CeO}_{2}$ addition. The increase of the resistance should be related to the low oxygen catalytic activity and total conductivity of $\mathrm{CeO}_{2}$-based materials. The surface exchange coefficient of $\mathrm{CeO}_{2}$-based materials is much lower than LSCF and LNO materials by several orders of magnitude $\left[{ }^{246-248}\right]$. Meanwhile, as reported by Zhu $\left[{ }^{249}\right]$, the total conductivity decreases significantly with the increase of the $\mathrm{CeO}_{2}$-based content in the cathode material. The values dropped from $115.5 \mathrm{~S} \mathrm{~cm}^{-1}$ to 11.6 rapidly when $50 \mathrm{wt}$ \% LDC was introduced into LSF cathode. Combining the original low electrical conductivity of LNO-based materials, the addition of $\mathrm{CeO}_{2}$-based materials could make the electrochemical performance of infiltration electrode deteriorates.

In the case of as-prepared co-infiltrated LSCF, equivalent circuit $\mathrm{R}_{5}\left(\mathrm{Q}_{1} \mathrm{R}_{1}\right)\left(\mathrm{Q}_{2} \mathrm{R}_{2}\right)\left(\mathrm{Q}_{3} \mathrm{R}_{3}\right)$ has been used to fit the EIS data and monitor the alternation of different elementary processes in different infiltration electrodes, where $R_{s}$ is the ohmic resistance, and $R_{n}$ and $\mathrm{Q}_{\mathrm{n}}$ the polarization resistance and constant phase element of different elementary processes, respectively. An equivalent capacitance value $\mathrm{Q}_{1}$ in the range of $10^{-3-} 10^{-2} \mathrm{~F} \mathrm{~cm}^{-2}$ is obtained from high frequency response. The considerable process is most probably the charge transfer process related with ionization reaction of adsorbed oxygen according to literature $\left[{ }^{94,215,250}\right]$. Based on the $\mathrm{Q}_{2}$ and characteristic frequency values, the oxygen surface adsorption/desorption and bulk oxygen diffusion processes are believed to relate with this intermediate behavior. In consideration of rapid oxygen transport properties of LSCF, LNO and $\mathrm{CeO}_{2}$, the resistance at the intermediate frequency should be attributed to the oxygen adsorption/desorption process. And an additional smaller arc at low frequency $10^{-1} \mathrm{~Hz}$ may be assigned to gas diffusion as reported elsewhere. In comparison with the pure LNOinfiltrated LSCF, both Rp at high and intermediate-frequency increase with increasing amount of $\mathrm{CeO}_{2}$. And the intermediate-frequency arcs gradually transfer to the rate limiting 
step, which is closely related to the surface areas. The significant increase in the intermediate-frequency resistance results from the reduced surface areas caused by flatted morphology and lower surface catalytic of $\mathrm{CeO}_{2}$.

After $1080 \mathrm{~h}$ heat treatment at $750^{\circ} \mathrm{C}$, both LNO-infiltrated LSCF and co-infiltrated LSCF showed increased $\mathrm{Rp}$ values. In the case of LNO-only sample, the cathode resistance increased from 0.02 to $0.53 \Omega \mathrm{cm}^{2}$ (26.5 times). In contrast, the co-infiltrated LSCF cathode exhibited a gradually enhanced stability as the addition of $\mathrm{CeO}_{2}$, with cathode resistance increased from 0.05 to 0.44 (8.8 times) for $10 \mathrm{~mol}^{\circ} \mathrm{CeO}_{2}$, and 0.12 to $0.61 \Omega$ $\mathrm{cm}^{2}$ (5.1 times) for $20 \mathrm{~mol} \% \mathrm{CeO}_{2}$ infiltrated samples. The much smaller intermediatefrequency resistance of the $10 \mathrm{~mol} . \% \mathrm{CeO}_{2} \& \mathrm{LNO}$ co-infiltrated LSCF indicates that the addition of $\mathrm{CeO}_{2}$ inhibits the particles growth of the infiltrated nanoparticles effectively. $\mathrm{A}$ much larger resistance at high-frequency in the LNO infiltrated LSCF when compared with the co-infiltrated sample could be caused by the strontium segregation on the LSCF backbone surface, since the delamination of LNO nanoparticles in the LNO-only sample leads to more bare LSCF surface which is not resistant to Sr segregation.

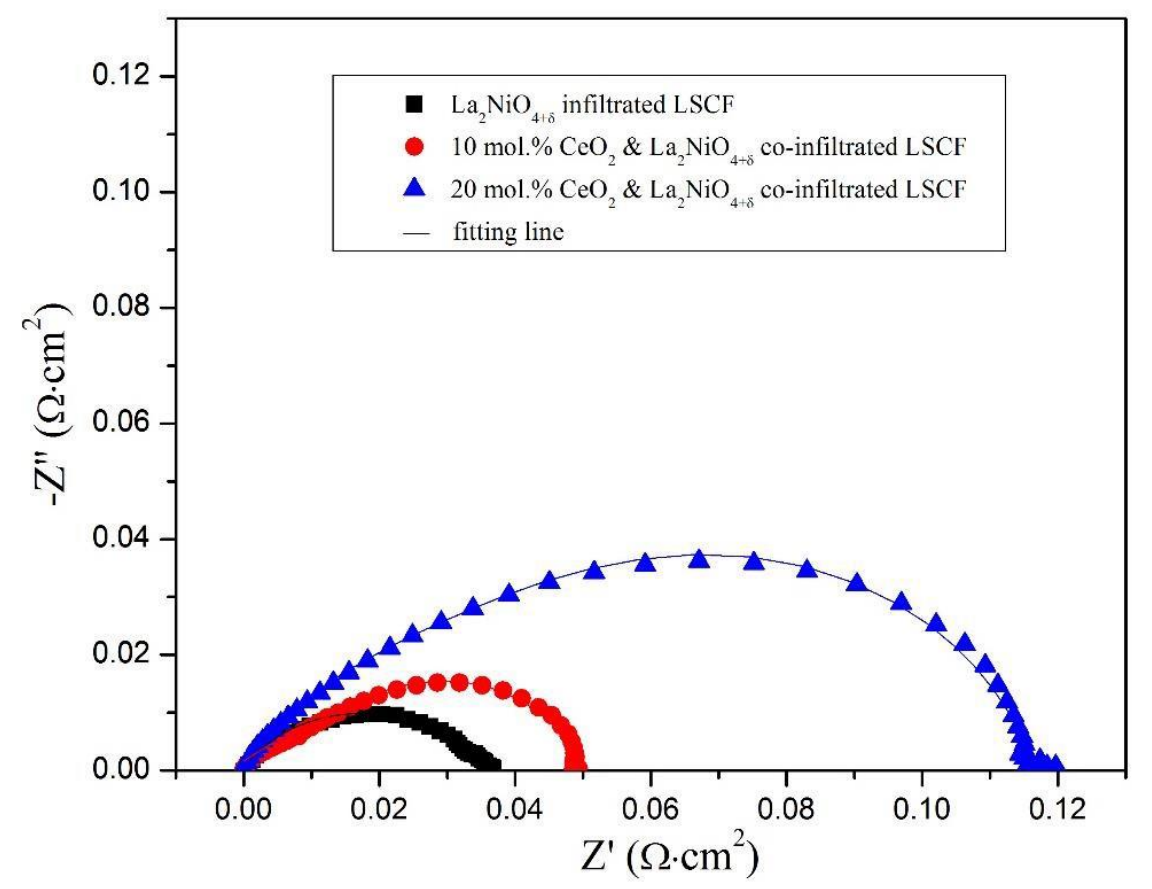

Figure 5.5 Nyquist diagram of electrochemical impedance spectra for LNO infiltrated LSCF symmetrical cells with and without $\mathrm{CeO}_{2}$ co-infiltration 


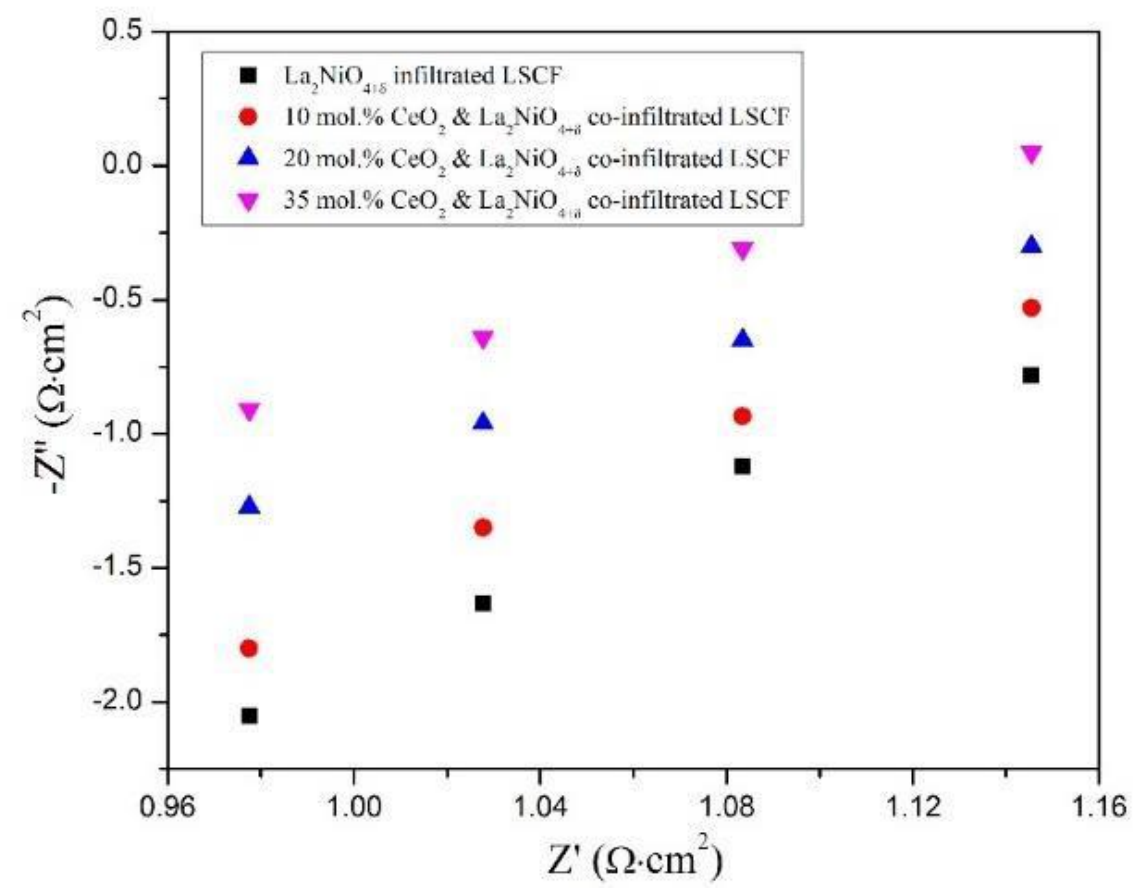

Figure 5.6 Arrhenius plots of fitted electrochemical impedance spectra for LNO infiltrated LSCF symmetrical cells with and without $\mathrm{CeO}_{2}$ co-infiltration

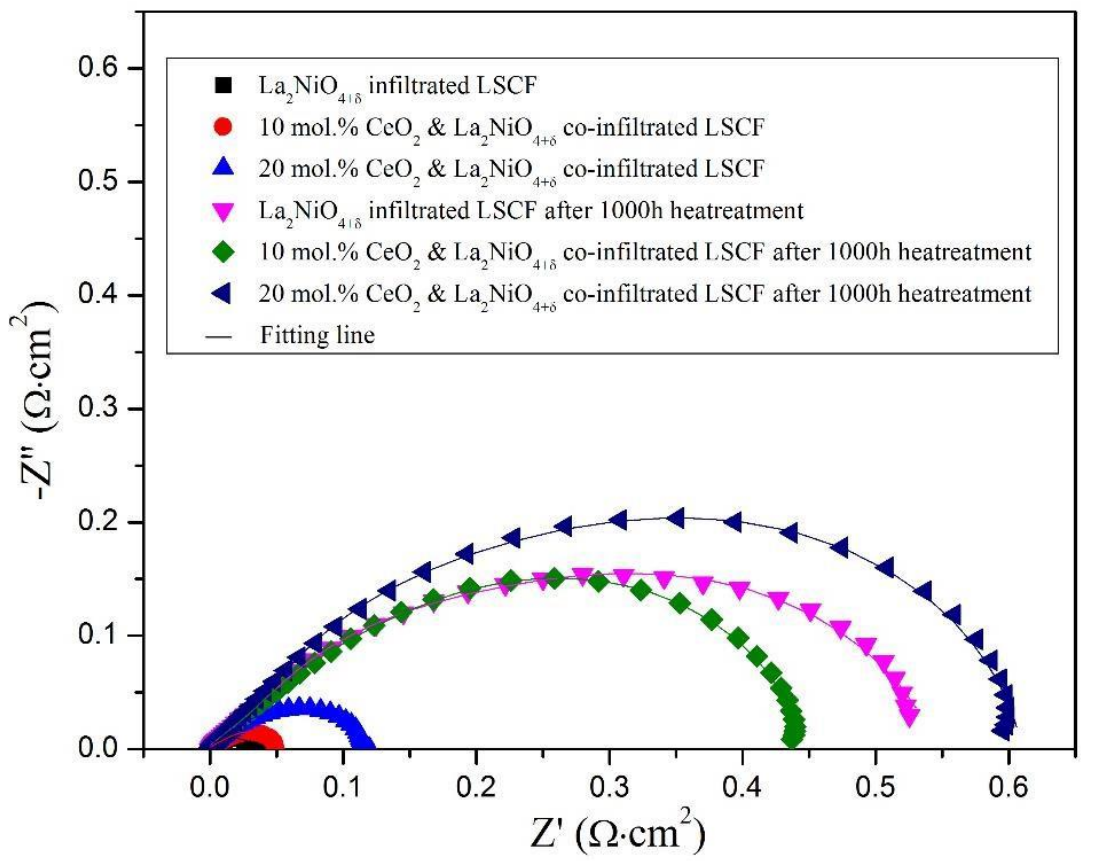

Figure 5.7 Nyquist diagram of electrochemical impedance spectra for LNO infiltrated LSCF symmetrical cells with and without $\mathrm{CeO}_{2}$ co-infiltration 


\subsection{Summary}

In this work, the chemically stable $\mathrm{CeO}_{2}$ with high melting point and good wettability, along with $\mathrm{La}_{2} \mathrm{NiO}_{4+\delta}$, was successfully co-infiltrated into LSCF backbone in one step. The characteristic peaks of $\mathrm{LNO}$ and $\mathrm{CeO}_{2}$ phase present in XRD patterns without impurity phase. The $\mathrm{CeO}_{2}$ addition in infiltration solution improve the contact between nanoparticles and LSCF backbone. Much flatter nano-layer was obtained in $\mathrm{CeO}_{2} \& \mathrm{LNO}$ co-infiltrated LSCF cathode, which enhance nanoparticles/backbone interface and reduce nanoparticles surface areas. And the cathode polarization resistance increases with increasing the amount of $\mathrm{CeO}_{2}$ addition in the infiltration solution because of reduced surface areas and lower catalytic and conductivity of $\mathrm{CeO}_{2}$. The cathode resistance of $20 \mathrm{~mol}$. \% $\mathrm{CeO}_{2} \&$ LNO coinfiltrated LSCF is still one magnitude less than pure LSCF. After $1080 \mathrm{~h}$ aging at $750^{\circ} \mathrm{C}$, the aggregation/growth and delamination of LNO can be observed in pure LNO-infiltrated LSCF cathode. The diameter of some isolated grown particles reach to 200-300nm, and much larger size of the compact particles with ambiguous boundary can be detected. In contrast, the $\mathrm{CeO}_{2}$ addition improve the wettability of infiltration solution between nanoparticles and LSCF backbone. Infiltrated nanoparticles are still attached on LSCF backbone tightly and uniformly. Compared with pure LNO infiltration, both less reduced surface areas and fewer exposed areas of LSCF with $\mathrm{CeO}_{2}$ addition allow the enhancement of cathode stability. Meanwhile, a part of nanoparticles kept the similar particle size with a diameter of 50-100nm after $1080 \mathrm{~h}$ aging, and a few nanoparticles surrounded by small particles grows to large particles with a diameter of less than $200 \mathrm{~nm}$. The particle size reaches steady state with a diameter of less than $200 \mathrm{~nm}$ and the particles growth process is completed. EIS data show that the degradation rate of $\mathrm{CeO}_{2} \& \mathrm{LNO}$ co-infiltrated LSCF cathode is much smaller than that of pure LNO infiltration. In comparison with as-prepared electrode, the cathode polarization resistance of pure LNO-infiltrated LSCF increases to over 26 times, but 5 times for infiltrated cathode with 20 mol. \% $\mathrm{CeO}_{2}$ addition. Both impedance measurements and SEM examinations suggested that addition of a second phases with higher melting point and good wettability are one potential way to decelerate particles growth and aggregation issues in infiltration electrode. 


\section{Chapter 6: Conclusions}

The $\mathrm{La}_{2} \mathrm{NiO}_{4+\delta}$ (LNO) nanoparticles have been successfully infiltrated into LSCF backbone to obtain a new hetero-structured SOFC cathode for ORR kinetic consideration. The currently comparative study of LSCF and LNO-infiltrated LSCF shows a significant reduction in polarization resistance and a $67 \%$ increase in maximum power density by using LNO-infiltrated LSCF cathode. The rapid surface and interface oxygen exchange properties of LNO/LSCF $\left(1.9 \times 10^{-4} \mathrm{~cm} / \mathrm{s}\right.$ and $3 \times 10-4 \mathrm{~cm} / \mathrm{s}$, respectively) was detected at

$700^{\circ} \mathrm{C}$ under air condition, which indicates that the incorporation rate of oxygen into LSCF is enhanced when using LNO/LSCF cathode. The benefits from the presence of the LNO nanoparticles demonstrate that LNO-infiltrated LSCF materials can act as high active surface oxygen exchange cathode with promising electrochemical properties and fast ORR behavior. The $\mathrm{Sr}$ and Co diffusion have been observed in our experiment, which is consistent with our assumption. A power density of $637 \mathrm{~mW} \cdot \mathrm{cm}^{-2}$ and a low degradation rate of $0.39 \%$ at a constant current density of $250 \mathrm{~mA} \cdot \mathrm{cm}^{-2}$ for the fuel cell using LNOinfiltrated LSCF cathode can be still achieved after long-term durability of about $500 \mathrm{~h}$ at $750^{\circ} \mathrm{C}$. Both ECR and LSCF self-infiltrated electrode results indicate that the performance improvement also results from the intrinsic LNO surface/ hetero-structured LSCF/LNO interface architecture offering more active reaction regions of $2 \mathrm{~PB}$ and $3 \mathrm{~PB}$, except for nanoscale LNO fine particles increasing surface active area. In consideration of relatively narrow gap between LSCF-infiltrated LSCF and LNO-infiltrated LSCF electrode resistance, the largest driving force for the increased performance may relate to the microstructure change with LNO fine particles introducing. Meanwhile, the cation segregation of LSCF could facilitate the surface catalytic properties and ORR kinetics of the LNO-infiltrated LSCF cathode with respect to favorable acceptor of LNO from Sr/Co doping. Although particle coarsening of LNO and cation diffusion from LSCF are observed, the preliminary stability results show a good long-term stability of the LNO-infiltrated LSCF cathode with a low degradation rate of $0.39 \%$ over $\sim 500 \mathrm{~h}$ at a constant current density of $250 \mathrm{~mA} \cdot \mathrm{cm}^{-2}$. The weak Sr peaks before and after polarization demonstrate the inhibition of LNO for $\mathrm{Sr}$ segregation. The nanoparticles aggregation/growth and lanthanum enrichment on surface 
can be observed in SEM images, which decrease either the effective surface reaction area or heterogeneous interface area and are responsible for the degradation of LNO-infiltrated LSCF cathode. Therefore, to further enhance the long-term stability of infiltrated ( $\left(\mathrm{La}_{0.6} \mathrm{Sr}_{0.4}\right)_{0.95} \mathrm{Co}_{0.2} \mathrm{Fe}_{0.8} \mathrm{O}_{3-8}$ (LSCF) cathode, two kinds of new hetero-structured cathodes, $\mathrm{La}_{2-\mathrm{x}} \mathrm{NiO}_{4+\delta}$-infiltrated LSCF and $\mathrm{CeO}_{2} \&$ LNO co-infiltrated LSCF, were developed. Firstly, A-site deficient LNO were prepared to inhibit the lanthanum segregation on the LNO-LSCF surface. The intrinsic properties ofLa $2-x \mathrm{NiO}_{4+\delta}(\mathrm{x}=0-0.2)$ were measured. The maximum deficient concentration of lanthanum is less than 0.2 in $\mathrm{La}_{2-x} \mathrm{NiO}_{4+\delta}$. The $\mathrm{La}_{2}-$ ${ }_{x} \mathrm{NiO}_{4+\delta}$ with higher deficient exhibits a slightly higher conductivity below $800^{\circ} \mathrm{C}$. Although the $\mathrm{La}_{2-x} \mathrm{NiO}_{4+\delta}$ with higher deficiency has higher conductivity, the surface charge transfer still dominates the impedance with increasing lanthanum deficiency. The surface charge transfer process for LNO materials is possibly controlled by surface interstitial oxygen concentration along with transition metal states. A one order of magnitude decrease in cathode polarization resistance after infiltration has been observed, and after $-0.2 \mathrm{~V}$ cathodic polarization for $960 \mathrm{~min}$, the degradation of $\mathrm{La}_{2-\mathrm{x}} \mathrm{NiO}_{4+\delta}(\mathrm{x}=0-0.2)$ coated LSCF reduces with increasing lanthanum deficient concentration. The enhanced stability of $\mathrm{La}_{2-\mathrm{x}} \mathrm{NiO}_{4+\delta}(\mathrm{x}=0-0.2)$ coated LSCF demonstrates that A-site deficient $\mathrm{La}_{2}$ ${ }_{x} \mathrm{NiO}_{4+\delta}$ is one potential infiltration materials for bare LSCF cathode to improve the surface stability.

The chemically stable $\mathrm{CeO}_{2}$ with high melting point and good wettability, along with $\mathrm{La}_{2} \mathrm{NiO}_{4+\delta}$, was successfully co-infiltrated into LSCF backbone in one step to suppress the nanoparticles aggregation/growth and delamination in infiltration electrode The cathode polarization resistance increases with the amount of $\mathrm{CeO}_{2}$ addition in the infiltration solution increasing because of reduced surface areas and lower catalytic and conductivity of $\mathrm{CeO}_{2}$. But the cathode resistance of $20 \mathrm{~mol} . \% \mathrm{CeO}_{2} \& \mathrm{LNO}$ co-infiltrated LSCF is still one magnitude less than pure LSCF. After $1080 \mathrm{~h}$ aging at $750^{\circ} \mathrm{C}$, the degradation rate of $\mathrm{CeO}_{2} \&$ LNO co-infiltrated LSCF cathode is smaller than that of pure LNO infiltration. In comparison with as-prepared electrode, the cathode polarization resistance of pure LNOinfiltrated LSCF increases to over 26 times, but 5 times for infiltrated cathode with $20 \mathrm{~mol}$. \% $\mathrm{CeO}_{2}$ addition. In LNO-only infiltration electrode, the LNO particles aggregate with each 
other and form a continuous network of solid nanoparticles and porosity with ambiguous grain boundary. The diameter of some isolated grown particles reach to $200-300 \mathrm{~nm}$, and much larger size of the compact particles with ambiguous boundary can be detected. In contrast, the $\mathrm{CeO}_{2}$ addition improve the wettability of infiltration solution between nanoparticles and LSCF backbone. Infiltrated nanoparticles are still attached on LSCF backbone tightly and uniformly. Meanwhile, a part of nanoparticles kept the similar particle size with a diameter of 50-100 $\mathrm{nm}$ after $1080 \mathrm{~h}$ aging, and a few nanoparticles surrounded by small particles grows to large particles with a diameter of less than $200 \mathrm{~nm}$. The particle size reaches steady state with a diameter of less than $200 \mathrm{~nm}$ and the particles growth process is completed. Hence, the existence of the second phase $\mathrm{CeO}_{2}$ nanoparticles suppress the atomic diffusion between LNO particles and further inhibits the nanoparticles growth/aggregation. It is beneficial for the control of the reduction of surface $2 \mathrm{~PB}$ and $3 \mathrm{~PB}$ area after long-term operation.

The enhanced stability of $\mathrm{La}_{2-x} \mathrm{NiO}_{4+\delta}(\mathrm{x}=0-0.2)$ and $\mathrm{CeO}_{2}$ \& LNO infiltrated LSCF demonstrates that A-site deficient $\mathrm{La}_{2-x} \mathrm{NiO}_{4+\delta}$ and $\mathrm{CeO}_{2}$-based materials are potential infiltration materials for bare LSCF cathode to improve the surface stability. 


\section{Reference}

1. Adler, S. B. Mechanism and kinetics of oxygen reduction on porous La1-xSrxCoO3- $\delta$ electrodes. Solid State Ionics (1998). doi:10.1016/S01672738(98)00179-9

2. Kubicek, M. et al. Electrochemical properties of La $0.6 \mathrm{Sr} 0.4 \mathrm{CoO} 3-\delta$ thin films investigated by complementary impedance spectroscopy and isotope exchange depth profiling. Solid State Ionics 256, 38-44 (2014).

3. Lee, D. et al. Oxygen surface exchange kinetics and stability of (La,Sr)2CoO4+/[small delta]/La1-xSrxMO3-[small delta] $(\mathrm{M}=\mathrm{Co}$ and $\mathrm{Fe})$ hetero-interfaces at intermediate temperatures. J. Mater. Chem. A 3, 2144-2157 (2015).

4. Lee, D. et al. Oxygen surface exchange kinetics and stability of (La,Sr)2CoO4+/[small delta]/La1-xSrxMO3-[small delta] $(\mathrm{M}=\mathrm{Co}$ and $\mathrm{Fe})$ hetero-interfaces at intermediate temperatures. J. Mater. Chem. A 3, 2144-2157 (2015).

5. Stambouli, A. B. Fuel cells: The expectations for an environmental-friendly and sustainable source of energy. Renew. Sustain. Energy Rev. 15, 4507-4520 (2011).

6. Stambouli, A. B. \& Traversa, E. Solid oxide fuel cells (SOFCs): A review of an environmentally clean and efficient source of energy. Renew. Sustain. Energy Rev. 6, 433-455 (2002).

7. Lee, T. S., Chung, J. N. \& Chen, Y. C. Design and optimization of a combined fuel reforming and solid oxide fuel cell system with anode off-gas recycling. Energy Convers. Manag. 52, 3214-3226 (2011).

8. Singhal, S. C. Science and Technology of Solid-Oxide Fuel Cells. MRS Bull. 25, 16-21 (2011).

9. Ormerod, R. M. Solid oxide fuel cells. Chem. Soc. Rev. 32, 17-28 (2003).

10. Brett, D. J. L. et al. Intermediate temperature solid oxide fuel cells. Chem. Soc. Rev. 37, 1568-78 (2008).

11. Kobayashi, Y. et al. Extremely High-efficiency Thermal Power System-Solid Oxide Fuel Cell (SOFC) Triple Combined-cycle System. Mitsubishi Heavy Ind. Tech. Rev. 48, 9-15 (2011). 
12. Brouwer, J. On the role of fuel cells and hydrogen in a more sustainable and renewable energy future. Curr. Appl. Phys. 10, S9-S17 (2010).

13. Ono, T., Miyachi, I., Suzuki, M. \& Higaki, K. Development of Residential SOFC Cogeneration System. IOP Conf. Ser. Mater. Sci. Eng. 18, 132007 (2011).

14. Sun, C. \& Stimming, U. Recent anode advances in solid oxide fuel cells. J. Power Sources 171, 247-260 (2007).

15. Mahato, N., Banerjee, A., Gupta, A., Omar, S. \& Balani, K. Progress in material selection for solid oxide fuel cell technology: A review. Prog. Mater. Sci. 72, 141337 (2015).

16. Chan, S. ., Low, C. . \& Ding, O. . Energy and exergy analysis of simple solid-oxide fuel-cell power systems. J. Power Sources 103, 188-200 (2002).

17. Mogensen, M. \& Skaarup, S. Kinetic and geometric aspects of solid oxide fuel cell electrodes. Solid State Ionics 86-88, 1151-1160 (1996).

18. Virkar, A. V., Chen, J., Tanner, C. W. \& Kim, J. W. Role of electrode microstructure on activation and concentration polarizations in solid oxide fuel cells. Solid State Ionics 131, 189-198 (2000).

19. Decorse, P., Caboche, G. \& Dufour, L. A comparative study of the surface and bulk properties of lanthanum-strontium-manganese oxides La 12 x $\mathrm{Sr}$ x MnO 3 6d as a function of Sr-content, oxygen potential and temperature. 117, 161-169 (1999).

20. Shaikh, S. P. S., Muchtar, A. \& Somalu, M. R. A review on the selection of anode materials for solid-oxide fuel cells. Renew. Sustain. Energy Rev. 51, 1-8 (2015).

21. Shri Prakash, B., Senthil Kumar, S. \& Aruna, S. T. Properties and development of Ni/YSZ as an anode material in solid oxide fuel cell: A review. Renew. Sustain. Energy Rev. 36, 149-179 (2014).

22. Koide, H. Properties of Ni/YSZ cermet as anode for SOFC. Solid State Ionics 132, 253-260 (2000).

23. Gong, M., Liu, X., Trembly, J. \& Johnson, C. Sulfur-tolerant anode materials for solid oxide fuel cell application. J. Power Sources 168, 289-298 (2007).

24. Laurencin, J., Delette, G., Morel, B., Lefebvre-Joud, F. \& Dupeux, M. Solid Oxide Fuel Cells damage mechanisms due to Ni-YSZ re-oxidation: Case of the Anode 
Supported Cell. J. Power Sources 192, 344-352 (2009).

25. Goodenough, J. B. \& Huang, Y.-H. Alternative anode materials for solid oxide fuel cells. J. Power Sources 173, 1-10 (2007).

26. Marina, O. a. \& Mogensen, M. High-temperature conversion of methane on a composite gadolinia-doped ceria-gold electrode. Appl. Catal. A Gen. 189, 117-126 (1999).

27. Huang, Y.-H. et al. Double-Perovskite Anode Materials Sr2MMoO6 (M = Co, Ni) for Solid Oxide Fuel Cells. Chem. Mater. 21, 2319-2326 (2009).

28. Sengodan, S. et al. Layered oxygen-deficient double perovskite as an efficient and stable anode for direct hydrocarbon solid oxide fuel cells. Nat. Mater. 14, 205-209 (2014).

29. Kharton, V. V., Marques, F. M. B. \& Atkinson, A. Transport properties of solid oxide electrolyte ceramics: A brief review. Solid State Ionics 174, 135-149 (2004).

30. Lee, J.-H. et al. Improvement of Grain-Boundary Conductivity of 8 mol YttriaStabilized Zirconia by Precursor Scavenging of Siliceous Phase. J. Electrochem. Soc. 147, 2822 (2000).

31. Badwal, S. Scandia-zirconia electrolytes for intermediate temperature solid oxide fuel cell operation. Solid State Ionics 136-137, 91-99 (2000).

32. Badwal, S. P. S. Zirconia-based solid electrolytes: microstructure, stability and ionic conductivity. Solid State Ionics 52, 23-32 (1992).

33. Mogensen, M. Physical, chemical and electrochemical properties of pure and doped ceria. Solid State Ionics 129, 63-94 (2000).

34. Inaba, H. Ceria-based solid electrolytes. Solid State Ionics 83, 1-16 (1996).

35. Goodenough, J. B., Ruiz-Diaz, J. E. \& Zhen, Y. S. Oxide-ion conduction in Ba , In205 and Ba, In , M08 ( M = Ce , Hf , or Zr ). Solid State Ionics 44, 21-31 (1990).

36. Shuk, P. Oxide ion conducting solid electrolytes based on Bi2O3. Solid State Ionics 89, 179-196 (1996).

37. Stevenson, J. W., Armstrong, T. R., McCready, D. E., Pederson, L. R. \& Weber, W. J. Processing and electrical properties of alkaline earth-doped lanthanum gallate. $J$. Electrochem. Soc. 144, 3613-3620 (1997). 
38. Huang, P. Superior Oxygen Ion Conductivity of Lanthanum Gallate Doped with Strontium and Magnesium. J. Electrochem. Soc. 143, 1644 (1996).

39. Berastegui, P., Hull, S., Garcı Garc1, F. J. \& Grins, J. A Structural Investigation of La2(GeO4)O and Alkaline-Earth-Doped La9.33(GeO4)6O2. J. Solid State Chem. 168, 294-305 (2002).

40. Arikawa, H., Nishiguchi, H., Ishihara, T. \& Takita, Y. Oxide ion conductivity in Srdoped La10Ge6O27 apatite oxide. Solid State Ionics 136-137, 31-37 (2000).

41. Pirzada, M., Grimes, R. W., Minervini, L., Maguire, J. F. \& Sickafus, K. E. Oxygen migration in A2B2O7 pyrochlores. Solid State Ionics 140, 201-208 (2001).

42. Chen, Y. et al. Impact of $\mathrm{Sr}$ segregation on the electronic structure and oxygen reduction activity of SrTi1-xFexO3 surfaces. Energy Environ. Sci. 5, 7979-7988 (2012).

43. Finsterbusch, M., Lussier, A., Schaefer, J. A. \& Idzerda, Y. U. Electrochemically driven cation segregation in the mixed conductor $\mathrm{La} 0.6 \mathrm{Sr} 0.4 \mathrm{Co} 0.2 \mathrm{Fe} 0.8 \mathrm{O} 3-\delta$. Solid State Ionics 212, 77-80 (2012).

44. Kubicek, M. et al. Cation diffusion in $\mathrm{La}(0.6) \operatorname{Sr}(0.4) \mathrm{CoO}(3-\delta)$ below $800{ }^{\circ} \mathrm{C}$ and its relevance for Sr segregation. Phys. Chem. Chem. Phys. 16, 2715-26 (2014).

45. Siskin, M. Degradation Mechanisms of La-Sr-Co-Fe SOFC cathodes. J. Anal. Appl. Pyrolysis 287, 173-196 (1983).

46. Adler, S. B., Lane, J. A. \& Steele, B. C. H. Electrode Kinetics of Porous Mixed Conducting Oxygen Electrodes. J. Electrochem. Soc. 143, 3554-3564 (1996).

47. Zhu, C., Liu, X., Yi, C., Yan, D. \& Su, W. Electrochemical performance of PrBaCo $2 \mathrm{O} 5+1$ layered perovskite as an intermediate-temperature solid oxide fuel cell cathode. 185, 193-196 (2008).

48. Li, W. et al. Enhanced surface exchange activity and electrode performance of $(\mathrm{La} 2-2 \mathrm{xSr} 2 \mathrm{x})(\mathrm{Ni1}-\mathrm{xMnx}) \mathrm{O} 4+\delta$ cathode for intermediate temperature solid oxide fuel cells. J. Power Sources 318, 178-183 (2016).

49. Li, W. et al. New mechanistic insight into the oxygen reduction reaction on Ruddlesden-Popper cathodes for intermediate-temperature solid oxide fuel cells. Phys. Chem. Chem. Phys. 18, 8502-11 (2016). 
50. Shao, Z. \& Haile, S. M. A high-performance cathode for the next generation of solid-oxide fuel cells. Nature 431, 170-173 (2004).

51. Lee, H. Y., Huang, K. \& Goodenough, J. B. Sr- and Ni-Doped LaCoO3 and LaFeO3 Perovskites. J. Electrochem. Soc. 145, 3220-3227 (1998).

52. Adler, S. Limitations of charge-transfer models for mixed-conducting oxygen electrodes. Solid State Ionics 135, 603-612 (2000).

53. Adler, S., Lane, J. \& Steele, B. Electrode Kinetics of Porous Mixed- Conducting Oxygen Electrodes. J. Electrochem. Soc. 143, 3554-3564 (1996).

54. Trimaille, I. et al. Yttria-Stabilized Zirconia. 144, 717-720 (1997).

55. Coffey, G. W., Pederson, L. R. \& Rieke, P. C. Competition Between Bulk and Surface Pathways in Mixed Ionic Electronic Conducting Oxygen Electrodes. $J$. Electrochem. Soc. 150, A1139 (2003).

56. Fleig, J. On the current-voltage characteristics of charge transfer reactions at mixed conducting electrodes on solid electrolytes. Phys. Chem. Chem. Phys. 7, 2027-2037 (2005).

57. Kopasakis, G., Brinson, T., Credle, S. \& Xu, M. A Theoretical Solid Oxide Fuel Cell Model for System Controls and Stability Design. Vol. 4 Cycle Innov. Electr. Power; Ind. Cogener. Manuf. Mater. Metall. 5, 565-575 (2006).

58. Wang, J. H., Liu, M. \& Lin, M. C. Oxygen reduction reactions in the SOFC cathode of Ag/CeO2. Solid State Ionics 177, 939-947 (2006).

59. Chan, S. H., Chen, X. J. \& Khor, K. a. Cathode Micromodel of Solid Oxide Fuel Cell. J. Electrochem. Soc. 151, A164 (2004).

60. Svensson, A. M. Mathematical Modeling of Oxygen Exchange and Transport in Air-Perovskite-Yttria-Stabilized Zirconia Interface Regions. J. Electrochem. Soc. 145, 1390 (1998).

61. Liu, M. Fundamental issues in modeling of mixed ionic-electronic conductors (MIECs). Solid State Ionics 118, 11-21 (1999).

62. Mizusaki, J. Nonstoichiometry, diffusion, and electrical properties of perovskitetype oxide electrode materials. Solid State Ionics 52, 79-91 (1992).

63. Boukamp, B. A. Fuel cells: The amazing perovskite anode. Nat. Mater. 2, 294-296 
(2003).

64. Trofimenko, N. E. \& Ullmann, H. Oxygen stoichiometry and mixed ionic-electronic conductivity of Sr1-aCeaFe1-bCobO3-x perovskite-type oxides. J. Eur. Ceram. Soc. 20, 1241-1250 (2000).

65. Ullmann, H. \& Trofimenko, N. Composition, structure and transport properties of perovskite-type oxides. Solid State Ionics 119, 1-8 (1999).

66. Ullmann, H., Trofimenko, N., Tietz, F., Stöver, D. \& Ahmad-Khanlou, A. Correlation between thermal expansion and oxide ion transport in mixed conducting perovskite-type oxides for SOFC cathodes. Solid State Ionics 138, 79-90 (2000).

67. Yokokawa, H., Sakai, N., Kawada, T. \& Dokiya, M. Thermodynamic stabilities of perovskite oxides for electrodes and other electrochemical materials. Solid State Ionics 52, 43-56 (1992).

68. Cheng, J., Navrotsky, A., Zhou, X.-D. \& Anderson, H. U. Enthalpies of Formation of LaMO3 Perovskites (M = Cr, Fe, Co, and Ni). J. Mater. Res. 20, 191-200 (2005).

69. Tanasescu, S., Totir, N. D. \& Neiner, D. Correlations between the nonstoichiometry and the thermodynamic data of perovskite-type compounds in the La-Sr-Mn-O system. J. Optoelectron. Adv. Mater. 3, 101-106 (2001).

70. Tanasescu, S., Totir, N. D. \& Marchidan, D. I. Thermodynamic data of the perovskite-type state electrochemical technique. Science (80-. ). 43, 3-9 (1998).

71. Tanasescu, S., Totir, N. D. \& Marchidan, D. I. Thermodynamic properties of some perovskite type oxides used as SOFC cathode materials. Solid State Ionics 119, 311315 (1999).

72. Dusastre, V. \& Kilner, J. A. Optimisation of composite cathodes for intermediate temperature SOFC applications. Solid State Ionics 126, 163-174 (1999).

73. Steele, B. C. H. Survey of materials selection for ceramic fuel cells: II. Cathodes and anodes. Solid State Ionics 86-88, 1223-1234 (1996).

74. Steele, B. Appraisal of Ce1-yGdyO2-y/2 electrolytes for IT-SOFC operation at $500^{\circ}$ C. Solid State Ionics 129, 95-110 (2000).

75. Kostogloudis, G. \& Ftikos, C. Properties of A-site-deficient La0.6Sr0.4Co0.2Fe08O3- $\delta$-based perovskite oxide. Solid State Ionics 126, 143-151 
(1999).

76. Haanappel, V. A. C., Mertens, J. \& Mai, A. Performance Improvement of (La,Sr)MnO[sub 3] and (La,Sr)(Co,Fe)O[sub 3]-Type Anode-Supported SOFCs. $J$. Fuel Cell Sci. Technol. 3, 263 (2006).

77. Uhlenbruck, S., Moskalewicz, T., Jordan, N., Penkalla, H. J. \& Buchkremer, H. P. Element interdiffusion at electrolyte-cathode interfaces in ceramic high-temperature fuel cells. Solid State Ionics 180, 418-423 (2009).

78. Mori, M. Mechanisms of Thermal Expansion and Shrinkage of $\mathrm{La} 0.8 \mathrm{Sr} 0.2 \mathrm{MnO} 3+\delta$ Perovskites with Different Densities during Thermal Cycling in Air. J. Electrochem. Soc. 152, A732-A739 (2005).

79. Jiang, S. P. \& Love, J. G. Origin of the initial polarization behavior of Sr-doped LaMnO3 for O2 reduction in solid oxide fuel cells. Solid State Ionics 138, 183-190 (2001).

80. Wang, W. \& Jiang, S. P. A mechanistic study on the activation process of (La, Sr)MnO3 electrodes of solid oxide fuel cells. Solid State Ionics 177, 1361-1369 (2006).

81. Jiang, S. P. \& Love, J. G. Observation of structural change induced by cathodic polarization on ( $\mathrm{La}, \mathrm{Sr}$ ) $\mathrm{MnO} 3$ electrodes of solid oxide fuel cells. Solid State Ionics 158, 45-53 (2003).

82. Mekki, A. X-ray photoelectron spectroscopy of $\mathrm{CeO} 2-\mathrm{Na} 2 \mathrm{O}-\mathrm{SiO} 2$ glasses. $J$. Electron Spectros. Relat. Phenomena 142, 75-81 (2005).

83. Grossin, D. \& Noudem, J. G. Synthesis of fine La0.8Sr0.2MnO3 powder by different ways. Solid State Sci. 6, 939-944 (2004).

84. Poirson, A., Decorse, P. \& Caboche, G. A Dilatometric Study of the La0.8Sr0.2MnO3 SIntering Behaviour. Solid State Ionics 99, (1997).

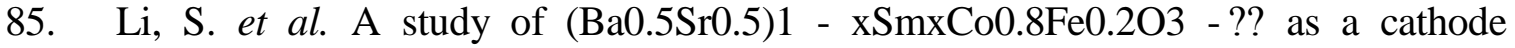
material for IT-SOFCs. J. Alloys Compd. 426, 408-414 (2006).

86. Raccah, P. M. \& Goodenough, J. B. A Localized-electron to collective-electron transition in the system (La, Sr)CoO3. J. Appl. Phys. 39, 1209-1210 (1968).

87. Petrov, A. N., Kononchuk, O. F., Andreev, A. V., Cherepanov, V. A. \& Kofstad, P. 
Crystal structure, electrical and magnetic properties of La1 - xSrxCoO3 - y. Solid State Ionics 80, 189-199 (1995).

88. Weber, A. \& Ivers-Tiffée, E. Materials and concepts for solid oxide fuel cells (SOFCs) in stationary and mobile applications. J. Power Sources 127, 273-283 (2004).

89. Riza, F., Ftikos, C., Tietz, F. \& Fischer, W. Preparation and characterization of Ln0.8Sr0.2Fe0.8Co0.2O3-x (Ln=La, Pr, Nd, Sm, Eu, Gd). J. Eur. Ceram. Soc. 21, 1769-1773 (2001).

90. Lee, K. T. \& Manthiram, A. Comparison of $\operatorname{Ln}(0.6) \operatorname{Sr}(0.4) \mathrm{CoO}$ (3-delta) ( $\mathrm{Ln}=\mathrm{La}$, $\mathrm{Pr}, \mathrm{Nd}, \mathrm{Sm}$, and Gd) as cathode materials for intermediate temperature solid oxide fuel cells. J. Electrochem. Soc. 153, A794-A798 (2006).

91. Yasumoto, K., Inagaki, Y., Shiono, M. \& Dokiya, M. An ( $\mathrm{La}, \mathrm{Sr})(\mathrm{Co}, \mathrm{Cu}) \mathrm{O} 3-$ ?? cathode for reduced temperature SOFCs. Solid State Ionics 148, 545-549 (2002).

92. Zhu, C. et al. Preparation and performance of $\mathrm{Pr} 0.7 \mathrm{Sr} 0.3 \mathrm{Co} 1-\mathrm{yCuyO} 3-\delta$ as cathode material of IT-SOFCs. Solid State Ionics 179, 1470-1473 (2008).

93. Chen, W., Wen, T., Nie, H. \& Zheng, R. Study of Ln0.6Sr0.4Co0.8Mn 0.2O3-?? $(\mathrm{Ln}=\mathrm{La}, \mathrm{Gd}, \mathrm{Sm}$ or $\mathrm{Nd})$ as the cathode materials for intermediate temperature SOFC. Mater. Res. Bull. 38, 1319-1328 (2003).

94. Fukunaga, H., Koyama, M., Takahashi, N., Wen, C. \& Yamada, K. Reaction model of dense $\mathrm{Sm} 0.5 \mathrm{Sr} 0.5 \mathrm{CoO} 3$ as SOFC cathode. Solid State Ionics 132, 279-285 (2000).

95. Pearson, D. J. \& Films, T. S. Intermediate Temperature Solid Oxide Fuel Cells Using a New LaGaO3 Based Oxide Ion Conductor. 145, 3177-3183 (1998).

96. Tu, H. Y., Takeda, Y., Imanishi, N. \& Yamamoto, O. $\operatorname{Ln}(1-\mathrm{x}) \operatorname{Sr}(\mathrm{x}) \mathrm{CoO}(3)(\mathrm{Ln}=\mathrm{Sm}$, Dy) for the electrode of solid oxide fuel cells. Solid State Ionics 100, 283-288 (1997).

97. Xia, C., Rauch, W., Chen, F. \& Liu, M. Sm0.5Sr0.5CoO3 cathodes for lowtemperature SOFCs. Solid State Ionics 149, 11-19 (2002).

98. Tietz, F. Thermal expansion of SOFC materials. Ionics (Kiel). 5, 129-139 (1999).

99. Ralph, J. M., Rossignol, C. \& Kumar, R. Cathode Materials for Reduced- 
Temperature SOFCs. J. Electrochem. Soc. 150, A1518 (2003).

100. Simner, S. P. et al. Development of lanthanum ferrite SOFC cathodes. J. Power Sources 113, 1-10 (2003).

101. Bongio, E. V et al. Microstructural and High-Temperature Electrical Characterization of La 1 - x Sr x FeO $3-\delta$. Fuel Cells 193-198 (2005).

102. Simner, S. P., Anderson, M. D., Pederson, L. R. \& Stevenson, J. W. Performance Variability of $\mathrm{La}(\mathrm{Sr}) \mathrm{FeO}[\mathrm{sub} 3] \mathrm{SOFC}$ Cathode with $\mathrm{Pt}, \mathrm{Ag}$, and $\mathrm{Au}$ Current Collectors. J. Electrochem. Soc. 152, A1851 (2005).

103. Coffey, G. et al. Copper doped lanthanum strontium ferrite for reduced temperature solid oxide fuel cells. Solid State Ionics 175, 73-78 (2004).

104. Simner, S., Anderson, M., Bonnett, J. \& Stevenson, J. Enhanced low temperature sintering of ( $\mathrm{Sr}, \mathrm{Cu})$-doped lanthanum ferrite SOFC cathodes. Solid State Ionics $\mathbf{1 7 5}$, 79-81 (2004).

105. Tsipis, E. V. \& Kharton, V. V. Electrode materials and reaction mechanisms in solid oxide fuel cells: A brief review: I Electrochemical behavior vs. materials science aspects. J. Solid State Electrochem. 12, 1367-1391 (2008).

106. Ferrite-based perovskites as cathode materials for anode-supported solid oxide fuel cells: Part I. Variation of composition. Solid State Ionics (2005).

107. Yokokawa, H., Tu, H., Iwanschitz, B. \& Mai, A. Fundamental mechanisms limiting solid oxide fuel cell durability. J. Power Sources 182, 400-412 (2008).

108. Tietz, F. et al. Materials Development for Advanced Planar Solid Oxide Fuel Cells. Int. J. Appl. Ceram. Technol. 445, 436-445 (2007).

109. Tietz, F., Mai, A. \& Stöver, D. From powder properties to fuel cell performance - A holistic approach for SOFC cathode development. Solid State Ionics 179, 15091515 (2008).

110. Tietz, F., Arul Raj, I., Zahid, M. \& St??ver, D. Electrical conductivity and thermal expansion of $\mathrm{La0} .8 \mathrm{Sr} 0.2(\mathrm{Mn}, \mathrm{Fe}, \mathrm{Co}) \mathrm{O} 3-? ?$ perovskites. Solid State Ionics 177, 1753-1756 (2006).

111. Meng, X., Lü, S., Ji, Y., Wei, T. \& Zhang, Y. Characterization of $\operatorname{Pr} 1-\mathrm{xSrxCo} 0.8 \mathrm{Fe} 0.2 \mathrm{O} 3-\delta(0.2 \leq \mathrm{x} \leq 0.6)$ cathode materials for intermediate- 
temperature solid oxide fuel cells. J. Power Sources 183, 581-585 (2008).

112. Adler, A. \& Stuart, B. 5/12/2016 EBSCOhost. i, 1-7 (2016).

113. Lane, J. Oxygen transport in $\mathrm{La} 0.6 \mathrm{Sr} 0.4 \mathrm{Co} 0.2 \mathrm{Fe} 0.8 \mathrm{O} 3-\delta$. Solid State Ionics 121, 201-208 (1999).

114. Lane, J. Measuring oxygen diffusion and oxygen surface exchange by conductivity relaxation. Solid State Ionics 136-137, 997-1001 (2000).

115. Mai, A. et al. Time-dependent performance of mixed-conducting SOFC cathodes. Solid State Ionics 177, 1965-1968 (2006).

116. Araki, W., Yamaguchi, T., Arai, Y. \& Malzbender, J. Strontium surface segregation in $\mathrm{La} 0.58 \mathrm{Sr} 0.4 \mathrm{Co} 0.2 \mathrm{Fe} 0.8 \mathrm{O} 3-\delta$ annealed under compression. Solid State Ionics $\mathbf{2 6 8}$, 1-6 (2014).

117. Liu, Y. et al. Performance stability and degradation mechanism of $\mathrm{La} 0.6 \mathrm{Sr} 0.4 \mathrm{Co} 0.2 \mathrm{Fe} 0.8 \mathrm{O} 3-\delta$ cathodes under solid oxide fuel cells operation conditions. Int. J. Hydrogen Energy 39, 15868-15876 (2014).

118. Zhao, L., Drennan, J., Kong, C., Amarasinghe, S. \& Jiang, S. P. Insight into surface segregation and chromium deposition on $\mathrm{La} 0.6 \mathrm{Sr} 0.4 \mathrm{Co} 0.2 \mathrm{Fe} 0.8 \mathrm{O} 3$-[small delta] cathodes of solid oxide fuel cells. J. Mater. Chem. A 2, 11114-11123 (2014).

119. Liu, R. R. et al. Influence of water vapor on long-term performance and accelerated degradation of solid oxide fuel cell cathodes. J. Power Sources 196, 7090-7096 (2011).

120. Liu, M. et al. Enhanced performance of LSCF cathode through surface modification. Int. J. Hydrogen Energy 37, 8613-8620 (2012).

121. Kim, J. Y., Sprenkle, V. L., Canfield, N. L., Meinhardt, K. D. \& Chick, L. A. Effects of Chrome Contamination on the Performance of $\mathrm{La}$ [sub 0.6$] \mathrm{Sr}[\mathrm{sub} 0.4] \mathrm{Co}[\mathrm{sub}$ 0.2]Fe[sub 0.8]O[sub 3] Cathode Used in Solid Oxide Fuel Cells. J. Electrochem. Soc. 153, A880 (2006).

122. Pan, Z., Liu, Q., Zhang, L., Zhang, X. \& Chan, S. H. Effect of Sr Surface Segregation of $\mathrm{La}_{0.6} \mathrm{Sr}{ }_{0.4} \mathrm{Co}{ }_{0.2} \mathrm{Fe}_{0.8} \mathrm{O}_{3-\delta}$ Electrode on Its Electrochemical Performance in SOC. J. Electrochem. Soc. 162, F1316-F1323 (2015).

123. Tietz, F., Haanappel, V. \& Mai, A. Performance of LSCF cathodes in cell tests. J. 
Power ... (2006).

124. Kharton, V. V, Viskup, A. P., Naumovich, E. N. \& Marques, F. M. B. Oxygen ion transport in La $2 \mathrm{NiO} 4$-based ceramics. J. Mater. Chem. 9, 2623-2629 (1999).

125. Takayanagi, T., Kogure, M. \& Terasaki, I. Single Crystals. J. Phys. Condens. Matter 14, 1361-1370 (2002).

126. Garcia, G., Burriel, M., Bonanos, N. \& Santiso, J. Electrical Conductivity and

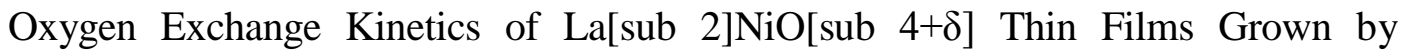
Chemical Vapor Deposition. J. Electrochem. Soc. 155, P28 (2008).

127. Butorin, S. et al. Electronic structure of $\mathrm{La} 2-x \mathrm{SrxNiO} 4+\delta$ studied by soft X-ray absorption and emission spectroscopies. Phys. C Supercond. 235-240, 1047-1048 (1994).

128. Munnings, C., Skinner, S., Amow, G., Whitfield, P. \& Davidson, I. Oxygen transport in the LaNiCoO system. Solid State Ionics 176, 1895-1901 (2005).

129. BOEHM, E. et al. Oxygen diffusion and transport properties in non-stoichiometric LnNiO oxides. Solid State Ionics 176, 2717-2725 (2005).

130. Villesuzanne, A., Demourgues, A., Grenier, J., Doumerc, J. \& Wattiaux, A. Band structure of La NiO ( La Ni O ). 91, 953-957 (1997).

131. Kim, G., Wang, S., Jacobson, a \& Chen, C. Measurement of oxygen transport kinetics in epitaxial $\mathrm{La} 2 \mathrm{NiO} 4+\delta$ thin films by electrical conductivity relaxation. Solid State Ionics 177, 1461-1467 (2006).

132. Zhao, H. et al. New cathode materials for ITSOFC: Phase stability, oxygen exchange and cathode properties of La2-xNiO4+ $\delta$. Solid State Ionics 179, 2000 2005 (2008).

133. Skinner, S. Oxygen diffusion and surface exchange in La2-xSrxNiO4+ $\delta$. Solid State Ionics 135, 709-712 (2000).

134. Kilner, J. A., De Souza, R. A. \& Fullarton, I. C. Surface exchange of oxygen in mixed conducting perovskite oxides. Solid State Ionics 86-88, 703-709 (1996).

135. Read, M. S. D., Islam, M. S., Watson, G. W. \& Hancock, F. E. Surface structures and defect properties of pure and doped La2NiO4. J. Mater. Chem. 11, 2597-2602 (2001). 
136. Kilner, J. A. \& Shaw, C. K. M. Mass transport in La2Ni1-xCoxO4+ $\delta$ oxides with the K2NiF4 structure. Solid State Ionics 154-155, 523-527 (2002).

137. Ferkhi, M., Ringued??, A., Khaled, A., Zerroual, L. \& Cassir, M. La 1.98Ni0 4????, a new cathode material for solid oxide fuel cell: Impedance spectroscopy study and compatibility with gadolinia-doped ceria and yttria-stabilized zirconia electrolytes. Electrochim. Acta 75, 80-87 (2012).

138. Tarancón, A., Burriel, M., Santiso, J., Skinner, S. J. \& Kilner, J. a. Advances in layered oxide cathodes for intermediate temperature solid oxide fuel cells. J. Mater. Chem. 20, 3799 (2010).

139. Choi, S., Yoo, S., Shin, J.-Y. \& Kim, G. High Performance SOFC Cathode Prepared by Infiltration of Lan $+1 \mathrm{NinO} 3 n+1(n=1,2$, and 3) in Porous YSZ. J. Electrochem. Soc. 158, B995 (2011).

140. Shin, W. S. Notice warning concerning copyright restrictions. Scand. J. For. Res. (2007).

141. Read, M. S. D., Islam, M. S., Watson, G. W. \& Hancock, F. E. Surface structures and defect properties of pure and doped La2NiO4. J. Mater. Chem. 11, 2597-2602 (2001).

142. Kammer, K. An EIS study of La2- x $\mathrm{Sr} x \mathrm{NiO} 4+\delta$ SOFC cathodes. Ionics (Kiel). (2009).

143. Zhao, H., Li, Q. \& Sun, L. Ln2MO4 cathode materials for solid oxide fuel cells. Sci. China Chem. 54, 898-910 (2011).

144. Caronna, T., Fontana, F., Natali Sora, I., Pelosato, R. \& Vigan??, L. Chemical compatibility of Sr-doped $\mathrm{La} 2 \mathrm{CuO} 4$ cathode material with LSGM solid oxide electrolyte. Solid State Ionics 181, 1355-1358 (2010).

145. Huang, X. et al. Preparation and characteristics of $\operatorname{Pr} 1.6 \mathrm{Sr} 0.4 \mathrm{NiO} 4+\mathrm{YSZ}$ as composite cathode of solid oxide fuel cells. J. Phys. Chem. Solids 70, 665-668 (2009).

146. Nie, H. W. et al. Preparation, thermal expansion, chemical compatibility, electrical conductivity and polarization of A2-??A?????MO4 $(\mathrm{A}=\mathrm{Pr}, \mathrm{Sm} ; \mathrm{A} ? ? ?=\mathrm{Sr} ; \mathrm{M}=$ Mn, Ni; ? $=0.3,0.6)$ as a new cathode for SOFC. Solid State Ionics 177, 1929- 
1932 (2006).

147. Cao, Y. et al. Preparation and characterization of $\mathrm{Nd} 2-\mathrm{xSrxCoO} 4+\delta$ cathodes for intermediate-temperature solid oxide fuel cell. Int. J. Hydrogen Energy 35, 55945600 (2010).

148. Aksenova, T. V., Gavrilova, L. Y., Yaremchenko, a. a., Cherepanov, V. a. \& Kharton, V. V. Oxygen nonstoichiometry, thermal expansion and high-temperature electrical properties of layered $\mathrm{NdBaCo} 2 \mathrm{O} 5+\delta$ and $\mathrm{SmBaCo} 2 \mathrm{O} 5+\delta$. Mater. Res. Bull. 45, 1288-1292 (2010).

149. Tsvetkov, D. S., Saricheva, N. S., Sereda, V. V. \& Zuev, A. Y. Oxygen Nonstoichiometry and Electrochemical Properties of $\mathrm{GdBaCo}[\mathrm{sub} 2-\mathrm{x}] \mathrm{Fe}[\mathrm{sub}$ x]O[sub 6- $\delta$ ] Double Perovskite Cathodes. J. Fuel Cell Sci. Technol. 8, 041006 (2011).

150. Tsvetkov, D. S., Sereda, V. V. \& Zuev, A. Y. Oxygen nonstoichiometry and defect structure of the double perovskite GdBaCo2O6 - ?? Solid State Ionics 180, 16201625 (2010).

151. Kim, Y. N. \& Manthiram, A. Layered $\mathrm{LnBaCo} 2-\mathrm{x} \mathrm{Cu} \times \mathrm{O} 5+\delta(\mathrm{x}=0 . .1)$ Perovskite Cathodes for Intermediate-Temperature Solid Oxide Fuel Cells. J. Electrochem. Soc. 158, B276-B282 (2011).

152. Kim, J.-H. et al. High Temperature Crystal Chemistry and Oxygen Permeation Properties of the Mixed Ionic-Electronic Conductors LnBaCo[sub 2]O[sub 5+ 5$]$ (Ln=Lanthanide). J. Electrochem. Soc. 156, B1376 (2009).

153. Chen, Y. C., Yashima, M., Peña-Martínez, J. \& Kilner, J. A. Experimental visualization of the diffusional pathway of oxide ions in a layered perovskite-type cobaltite PrBaCo2O5+ $\delta$. Chem. Mater. 25, 2638-2641 (2013).

154. Taranc??n, A., Marrero-L??pez, D., Pe??a-Mart??nez, J., Ruiz-Morales, J. C. \& N????ez, P. Effect of phase transition on high-temperature electrical properties of GdBaCo2O5 + x layered perovskite. Solid State Ionics 179, 611-618 (2008).

155. Lo Presti, L. et al. Crystal structure and structural phase transitions in the GdBaCo $\langle$ math display='inline' $\rangle\langle$ msub $\rangle\langle$ mrow $/\rangle\langle\mathrm{mn}\rangle 2</ \mathrm{mn}\rangle\langle$ msub $\rangle\langle$ math $\rangle \mathrm{O}$ $\langle$ math display='inline' $>\langle$ msub $\rangle\langle$ mrow $/\rangle\langle$ mrow $\rangle\langle\mathrm{mn}\rangle 5.0\langle/ \mathrm{mn}\rangle\langle$ mrow $\rangle$ 
</msub > </math> cobaltite. Phys. Rev. B 84, 104107 (2011).

156. Aksenova, T. V., Gavrilova, L. Y., Tsvetkov, D. S., Voronin, V. I. \& Cherepanov, V. a. Crystal structure and physicochemical properties of layered perovskite-like phases LnBaCo2O5 + $\delta$. Russ. J. Phys. Chem. A 85, 427-432 (2011).

157. Taskin, A. A., Lavrov, A. N. \& Ando, Y. Transport and magnetic properties of GdBa Co2 O5+x single crystals: A cobalt oxide with square-lattice Co O2 planes over a wide range of electron and hole doping. Phys. Rev. B - Condens. Matter Mater. Phys. 71, 1-28 (2005).

158. Zhang, K., Ge, L., Ran, R., Shao, Z. \& Liu, S. Synthesis, characterization and evaluation of cation-ordered $\mathrm{LnBaCo} 2 \mathrm{O} 5+?$ ? as materials of oxygen permeation membranes and cathodes of SOFCs. Acta Mater. 56, 4876-4889 (2008).

159. Taranc??n, A. et al. Stability, chemical compatibility and electrochemical performance of $\mathrm{GdBaCo} 2 \mathrm{O} 5+\mathrm{x}$ layered perovskite as a cathode for intermediate temperature solid oxide fuel cells. Solid State Ionics 179, 2372-2378 (2008).

160. van Doorn, R. Kinetic decomposition of $\mathrm{La} 0.3 \mathrm{Sr} 0.7 \mathrm{CoO} 3-\delta$ perovskite membranes during oxygen permeation. Solid State Ionics 111, 263-272 (1998).

161. Eth, D. W. Oxygen Reduction at Cathodes of Solid Oxide Fuel Cells presented by Reduction. Interchange (1966).

162. Sase, M. et al. Enhancement of oxygen exchange at the hetero interface of (La,Sr)CoO3/(La,Sr)2CoO4 in composite ceramics. Solid State Ionics 178, 1843 1852 (2008).

163. Sase, M. et al. Enhancement of Oxygen Surface Exchange at the Hetero-interface of $(\mathrm{La}, \mathrm{Sr}) \mathrm{CoO}[\mathrm{sub}$ 3]/(La,Sr)[sub 2] CoO[sub 4] with PLD-Layered Films. $J$. Electrochem. Soc. 155, B793 (2008).

164. Kushima, A. et al. Interstitialcy diffusion of oxygen in tetragonal $\mathrm{La} 2 \mathrm{CoO} 4+\delta$. Phys. Chem. Chem. Phys. 13, 2242-2249 (2011).

165. Zhou, J. et al. Density functional theory study on oxygen adsorption in LaSrCoO4: An extended cathode material for solid oxide fuel cells. Appl. Surf. Sci. 258, $3133-$ 3138 (2012).

166. Hong, W. T. et al. Tuning the Spin State in LaCoO 3 Thin Films for Enhanced 
High- Temperature Oxygen Electrocatalysis. J. Phys. Chem. Lett. 4, 2493-2499 (2013).

167. Tsvetkov, N., Chen, Y. \& Yildiz, B. Reducibility of Co at the $\mathrm{La} 0.8 \mathrm{Sr} 0.2 \mathrm{CoO} 3 /(\mathrm{La} 0.5 \mathrm{Sr} 0.5) 2 \mathrm{CoO} 4$ hetero-interface at elevated temperatures. $J$. Mater. Chem. A 2, 14690-14695 (2014).

168. Ma, W. et al. Vertically aligned nanocomposite $\mathrm{La} 0.8 \mathrm{Sr} 0.2 \mathrm{CoO} 3 /(\mathrm{La} 0.5 \mathrm{Sr} 0.5$ ) $2 \mathrm{CoO} 4$ cathodes - electronic structure, surface chemistry and oxygen reduction kinetics. J. Mater. Chem. A 3, 207-219 (2015).

169. Lee, D. et al. Strontium influence on the oxygen electrocatalysis of $\mathrm{La} 2-\mathrm{xSrxNiO} 4 \pm \delta(0.0 \leq \mathrm{xSr} \leq 1.0)$ thin films. J. Mater. Chem. A 2, 6480 (2014).

170. Crumlin, E. J. et al. Oxygen Electrocatalysis on Epitaxial La0.6Sr0.4CoO3Perovskite Thin Films for Solid Oxide Fuel Cells. J. Electrochem. Soc. 159, F219F225 (2012).

171. Warning, N. \& Copyright, C. DocuServe.

172. Fallis, A. . No Title No Title. J. Chem. Inf. Model. 53, 1689-1699 (2013).

173. a B. 519, 2012 (2012).

174. Feng, Z. et al. Anomalous Interface and Surface Strontium Segregation in (La 1- y Sr y ) $2 \mathrm{CoO} 4 \pm \delta / \mathrm{La} 1-\mathrm{x} \mathrm{Sr}$ x $\mathrm{CoO} 3-\delta$ Heterostructured Thin Films. J. Phys. Chem. Lett. 5, 1027-1034 (2014).

175. Transactions, E. C. S. \& Society, T. E. Surface Chemistry and Non-Stoichiometry of $\mathrm{Nd} 2 \mathrm{NiO} 4+.57,1743-1752$ (2013).

176. La, À. \& Coo, À. Terms of Use Tensile Lattice Strain Accelerates Oxygen Surface Exchange and. 3276-3286 (2013).

177. Stoerzinger, K. a., Choi, W. S., Jeen, H., Lee, H. N. \& Shao-Horn, Y. Role of Strain and Conductivity in Oxygen Electrocatalysis on $\mathrm{LaCoO} \quad 3 \quad$ Thin Films. J. Phys. Chem. Lett. 6, 487-492 (2015).

178. Zhou, J., Chen, G., Wu, K. \& Cheng, Y. Interaction of La 2 NiO 4 (100) Surface with Oxygen Molecule: A First-Principles Study. J. Phys. Chem. C 117, 1299112999 (2013).

179. Transactions, E. C. S. \& Society, T. E. C to intermediate range (500 to 700. 58, $265-$ 
274 (2013).

180. Han, J. W. \& Yildiz, B. Mechanism for enhanced oxygen reduction kinetics at the $(\mathrm{La}, \mathrm{Sr}) \mathrm{CoO} 3-\delta /(\mathrm{La}, \mathrm{Sr}) 2 \mathrm{CoO} 4+\delta$ hetero-interface. Energy Environ. Sci. 5, 8598 (2012).

181. Tsvetkov, N., Lu, Q., Chen, Y. \& Yildiz, B. Accelerated Oxygen Exchange Kinetics on Nd $2 \mathrm{NiO} 4$ p $\delta$ Thin Films with Tensile Strain along c - Axis. (2015).

182. Lee, D. et al. Strain In fl uence on the Oxygen Electrocatalysis of the (100)-Oriented Epitaxial La $2 \mathrm{NiO} 4+$. (2013).

183. Abstract \# 1902 , Honolulu PRiME 2012 , (C) 2012 The Electrochemical Society. 17696 (2012).

184. Cai, Z., Kubicek, M., Fleig, J. \& Yildiz, B. Chemical Heterogeneities on La 0.6 Sr $0.4 \mathrm{CoO} 3-\delta$ Thin Films - Correlations to Cathode Surface Activity and Stability. Chem. Mater. 24, 1116-1127 (2012).

185. Feng, Z. et al. Perovskite Thin Films. (2013).

186. Warning, N. \& Copyright, C. Notice warning concerning copyright restrictions.

187. Crumlin, E. J. et al. Oxygen Electrocatalysis on Epitaxial La0.6Sr0.4CoO3Perovskite Thin Films for Solid Oxide Fuel Cells. J. Electrochem. Soc. 159, F219F225 (2012).

188. Lee, D. et al. Strontium influence on the oxygen electrocatalysis of $\mathrm{La} 2-\mathrm{xSrxNiO} 4 \pm \delta(0.0 \leq \mathrm{xSr} \leq 1.0)$ thin films. J. Mater. Chem. A 2, 6480 (2014).

189. Burriel, M. et al. Anisotropic oxygen diffusion properties in epitaxial thin films of La2NiO4+ס. J. Mater. Chem. 18, 416 (2008).

190. Vohs, J. M. \& Gorte, R. J. High-performance SOFC cathodes prepared by infiltration. Adv. Mater. 21, 943-956 (2009).

191. Jiang, S. P. Nanoscale and nano-structured electrodes of solid oxide fuel cells by infiltration: Advances and challenges. Int. J. Hydrogen Energy 37, 449-470 (2012).

192. Jiang, Z., Xia, C. \& Chen, F. Nano-structured composite cathodes for intermediatetemperature solid oxide fuel cells via an infiltration/impregnation technique. Electrochim. Acta 55, 3595-3605 (2010).

193. Jiang, S. P. A review of wet impregnation - An alternative method for the fabrication 
of high performance and nano-structured electrodes of solid oxide fuel cells. Mater. Sci. Eng. A 418, 199-210 (2006).

194. Kim, J.-S. et al. Highly Active and Thermally Stable Core-Shell Catalysts for Solid Oxide Fuel Cells. J. Electrochem. Soc. 158, B596 (2011).

195. Jiang, S. P. \& Wang, W. Fabrication and performance of GDC-impregnated (La,Sr)MnO3 cathodes for intermediate temperature solid oxide fuel cells. $J$. Electrochem. Soc. 152, A1398-A1408 (2005).

196. Zhao, E. et al. Electrospinning La0.8Sr0.2Co0.2Fe0.8O3- $\delta$ tubes impregnated with Ce0.8Gd0.2O1.9 nanoparticles for an intermediate temperature solid oxide fuel cell cathode. Int. J. Hydrogen Energy 38, 6821-6829 (2013).

197. Hansen, K. K., Wandel, M., Liu, Y. L. \& Mogensen, M. Effect of impregnation of $\mathrm{La} 0.85 \mathrm{Sr} 0.15 \mathrm{MnO} 3 / \mathrm{yttria}$ stabilized zirconia solid oxide fuel cell cathodes with La0.85Sr0.15MnO3 or Al2O3 nano-particles. Electrochim. Acta 55, 4606-4609 (2010).

198. Leng, Y. J., Chan, S. H., Khor, K. A. \& Jiang, S. P. Development of LSM / YSZ composite cathode for anode-supported solid oxide fuel cells. J. Appl. electochemistry 3, 409-415 (2004).

199. Jiang, S. P., Leng, Y. J., Chan, S. H. \& Khor, K. A. Development of (La,Sr)MnO[sub 3]-Based Cathodes for Intermediate Temperature Solid Oxide Fuel Cells. Electrochem. Solid-State Lett. 6, A67 (2003).

200. Chen, K., Ai, N. \& Jiang, S. P. Development of (Gd, Ce) O2-Impregnated ( La, Sr) MnO3 Anodes of High Temperature Solid Oxide Electrolysis Cells. J. Electrochem. Soc. 157, P89-P94 (2010).

201. Jiang, Z., Zhang, L., Cai, L. \& Xia, C. Bismuth oxide-coated (La,Sr)MnO3 cathodes for intermediate temperature solid oxide fuel cells with yttria-stabilized zirconia electrolytes. Electrochim. Acta 54, 3059-3065 (2009).

202. Huang, Y., Ahn, K., Vohs, J. M. \& Gorte, R. J. Characterization of Sr-Doped LaCoO[sub 3]-YSZ Composites Prepared by Impregnation Methods. J. Electrochem. Soc. 151, A1592 (2004).

203. Shah, M., Voorhees, P. W. \& Barnett, S. A. Time-dependent performance changes 
in LSCF-infiltrated SOFC cathodes: The role of nano-particle coarsening. Solid State Ionics 187, 64-67 (2011).

204. Zhu, X. et al. Development of La0.6Sr0.4Co0.2Fe 0.8O3- $\delta$ cathode with an improved stability via La0.8Sr0.2MnO3-film impregnation. Int. J. Hydrogen Energy 38, 5375-5382 (2013).

205. Wang, W., Gross, M. D., Vohs, J. M. \& Gorte, R. J. The Stability of LSF-YSZ Electrodes Prepared by Infiltration. J. Electrochem. Soc. 154, B439 (2007).

206. Liu, Y. et al. A stability study of impregnated LSCF-GDC composite cathodes of solid oxide fuel cells. J. Alloys Compd. 578, 37-43 (2013).

207. Buyukaksoy, A., Petrovsky, V. \& Dogan, F. Stability and Performance of Solid Oxide Fuel Cells with Nanocomposite Electrodes. J. Electrochem. Soc. 159, B666 (2012).

208. Zhang, Y. \& Xia, C. A durability model for solid oxide fuel cell electrodes in thermal cycle processes. J. Power Sources 195, 6611-6618 (2010).

209. Zhao, F., Peng, R. \& Xia, C. A La0.6Sr0.4CoO3-??-based electrode with high durability for intermediate temperature solid oxide fuel cells. Mater. Res. Bull. 43, 370-376 (2008).

210. Lynch, M. E. et al. Enhancement of La0.6Sr0.4Co0.2Fe0.8O3- $\delta$ durability and surface electrocatalytic activity by $\mathrm{La} 0.85 \mathrm{Sr} 0.15 \mathrm{MnO} 3 \pm \delta$ investigated using a new test electrode platform. Energy Environ. Sci. 4, 2249 (2011).

211. Chun, W. C. Lawrence Berkeley National Laboratory Lawrence Berkeley National Laboratory. (2014).

212. Sholklapper, T. Z., Jacobson, C. P., Visco, S. J. \& Dejonghe, L. C. Synthesis of dispersed and contiguous nanoparticles in solid oxide fuel cell electrodes. Fuel Cells 8, 303-312 (2008).

213. Yamahara, K., Jacobson, C. P., Visco, S. J. \& De Jonghe, L. C. Catalyst-infiltrated supporting cathode for thin-film SOFCs. Solid State Ionics 176, 451-456 (2005).

214. He, F., Wu, T., Peng, R. \& Xia, C. Cathode reaction models and performance analysis of $\mathrm{Sm} 0.5 \mathrm{Sr} 0.5 \mathrm{CoO} 3-$ ??-BaCe0.8Sm0.2O3-?? composite cathode for solid oxide fuel cells with proton conducting electrolyte. J. Power Sources 194, 263-268 
(2009).

215. Shah, M. \& Barnett, S. A. Solid oxide fuel cell cathodes by infiltration of La0.6Sr0.4Co0.2Fe0.8O3 - ?? into Gd-Doped Ceria. Solid State Ionics 179, 2059 2064 (2008).

216. Li, Y., Gerdes, K., Diamond, H. \& Liu, X. An improved method to increase the predictive accuracy of the ECR technique. Solid State Ionics 204-205, 104-110 (2011).

217. No Title. (2012).

218. Li, Y., Gerdes, K., Horita, T. \& Liu, X. Surface Exchange and Bulk Diffusivity of LSCF as SOFC Cathode: Electrical Conductivity Relaxation and Isotope Exchange Characterizations. J. Electrochem. Soc. 160, F343-F350 (2013).

219. Chen, Y. et al. Advances in Cathode Materials for Solid Oxide Fuel Cells: Complex Oxides without Alkaline Earth Metal Elements. Adv. Energy Mater. 5, (2015).

220. Gao, Z., Mogni, L. V., Miller, E. C., Railsback, J. G. \& Barnett, S. A. A perspective on low-temperature solid oxide fuel cells. Energy Environ. Sci. 9, 1602-1644 (2016).

221. Druce, J. et al. Surface termination and subsurface restructuring of perovskite-based solid oxide electrode materials. Energy Environ. Sci. 7, 3893-3599 (2014).

222. Druce, J., Ishihara, T. \& Kilner, J. Surface composition of perovskite-type materials studied by Low Energy Ion Scattering (LEIS). Solid State Ionics 262, 893-896 (2014).

223. Burriel, M. et al. Absence of $\mathrm{Ni}$ on the outer surface of $\mathrm{Sr}$ doped La2NiO4 single crystals. Energy Environ. Sci. 7, 311 (2014).

224. Burriel, M. et al. Influence of Crystal Orientation and Annealing on the Oxygen Diffusion and Surface Exchange of La $2 \mathrm{NiO} 4+\delta$. J. Phys. Chem. C 120, 1792717938 (2016).

225. Wu, J., Pramana, S. S., Skinner, S. J., Kilner, J. a \& Horsfield, A. P. Why Ni is absent from the surface of $\mathrm{La} 2 \mathrm{NiO} 4+\delta$ ? J. Mater. Chem. A 3, 23760-23767 (2015).

226. Vashook, V. . et al. Oxygen nonstoichiometry and electrical conductivity of the solid solutions La2-xSrxNiOy $(0 \leq x \leq 0.5)$. Solid State Ionics 110, 245-253 (1998). 
227. Huang, D. P., Xu, Q., Chen, W., Zhang, F. \& Liu, H. X. Sintering, microstructure and conductivity of La2NiO4+?? ceramic. Ceram. Int. 34, 651-655 (2008).

228. Shen, Y., Zhao, H., Liu, X. \& Xu, N. Preparation and electrical properties of Cadoped $\mathrm{La}(2) \mathrm{NiO}(4+\delta)$ cathode materials for IT-SOFC. Phys. Chem. Chem. Phys. 12, 15124-31 (2010).

229. Minh, N. Q. Solid oxide fuel cell technology — features and applications. 174, 271277 (2004).

230. Zhang, X., Zhang, H. \& Liu, X. High performance La2NiO4 $+\delta$-infiltrated (La0.6Sr0.4)0.995 Co0.2Fe0.8O3- $\delta$ cathode for solid oxide fuel cells. J. Power Sources 269, 412-417 (2014).

231. Liu, Y., Chi, B., Pu, J. \& Li, J. Performance degradation of impregnated $\mathrm{La} 0.6 \mathrm{Sr} 0.4 \mathrm{Co} 0.2 \mathrm{Fe} 0.8 \mathrm{O} 3+\mathrm{Y} 2 \mathrm{O} 3$ stabilized $\mathrm{ZrO} 2$ composite cathodes of intermediate temperature solid oxide fuel cells. Int. J. Hydrogen Energy 37, 43884393 (2012).

232. Zhou, Y. et al. Performance and degradation of metal-supported solid oxide fuel cells with impregnated electrodes. Int. J. Hydrogen Energy 39, 2279-2285 (2014).

233. Lee, S., Miller, N. \& Gerdes, K. Long-Term Stability of SOFC Composite Cathode Activated by Electrocatalyst Infiltration. J. Electrochem. Soc. 159, F301-F308 (2012).

234. Chen, J. et al. Performance of large-scale anode-supported solid oxide fuel cells with impregnated $\mathrm{La} 0.6 \mathrm{Sr} 0.4 \mathrm{Co} 0.2 \mathrm{Fe} 0.8 \mathrm{O} 3-? ?+\mathrm{Y} 2 \mathrm{O} 3$ stabilized $\mathrm{ZrO} 2$ composite cathodes. J. Power Sources 195, 5201-5205 (2010).

235. De Jonghe, L. C. \& Rahaman, M. N. Sintering of Ceramics. Handbook of Advanced Ceramics: Materials, Applications, Processing and Properties 1-2, 187-264 (2003).

236. German, R. M. Fundamentals of sintering. ASM Int. Eng. Mater. Handb. 4, 260-269 (1991).

237. Farrell, H. H. \& Van Siclen, C. D. Binding energy, vapor pressure, and melting point of semiconductor nanoparticles. J. Vac. Sci. Technol. B Microelectron. Nanom. Struct. 25, 1441 (2007).

238. Olevsky, Eugene A (San Diego State University, California, U. Sintering theory: a 
Brief Introduction. Present. Tech. Univ. Darmstadt (2011).

239. Tian, C., Du, Y. \& Chan, S.-W. Preparation and microstructural study of $\mathrm{CeO} 2$ thin films. J. Vac. Sci. Technol. A Vacuum, Surfaces, Film. 15, 85-92 (1997).

240. Nguyen, T. L. et al. Preparation and evaluation of doped ceria interlayer on supported stabilized zirconia electrolyte SOFCs by wet ceramic processes. Solid State Ionics 174, 163-174 (2004).

241. Shiono, M. et al. Effect of $\mathrm{CeO} 2$ interlayer on $\mathrm{ZrO} 2$ electrolyte/La(Sr)CoO3 cathode for low-temperature SOFCs. Solid State Ionics 170, 1-7 (2004).

242. Lou, X. et al. Controlling the morphology and uniformity of a catalyst-infiltrated cathode for solid oxide fuel cells by tuning wetting property. J. Power Sources 195, 419-424 (2010).

243. Mi, X. et al. Synthesis and luminescence of Ca $9 \mathrm{Eu} 1 \mathrm{À} x \operatorname{Ln} \times(\mathrm{VO} 4) 7(\mathrm{Ln}=\mathrm{Y}$, La , Gd , Lu ) phosphors. 60, 72-78 (2014).

244. Mori, T., Drennan, J., Lee, J. H., Li, J. G. \& Ikegami, T. Oxide ionic conductivity and microstructures of Sm- or La-doped CeO2-based systems. Solid State Ionics 154-155, 461-466 (2002).

245. Sase, M. et al. Enhancement of Oxygen Surface Exchange at the Hetero-interface of (La,Sr)CoO3/(La,Sr)2CoO4 with PLD-Layered Films. 155, 4-9 (2008).

246. Armstrong, E. N., Duncan, K. L., Oh, D. J., Weaver, J. F. \& Wachsman, E. D. Determination of Surface Exchange Coefficients of LSM, LSCF, YSZ, GDC Constituent Materials in Composite SOFC Cathodes. J. Electrochem. Soc. 158, B492 (2011).

247. De Souza, R. Oxygen transport in La1-xSrxMn1-yCoyO3 $\pm \delta$ perovskites Part I. Oxygen tracer diffusion. Solid State Ionics 106, 175-187 (1998).

248. Sirman, J. D. \& Kilner, J. A. with La._SrFeCo1 -y³. 143, 229-231 (1996).

249. Sun, S., Zhu, X. \& Yuan, Y. Electrochemical properties of $\mathrm{La} 0.8 \mathrm{Sr} 0.2 \mathrm{FeO} 3-\delta-$ $\mathrm{La} 0.45 \mathrm{Ce} 0.55 \mathrm{O} 2-\delta$ composite cathodes for intermediate temperature SOFC. $J$. Solid State Electrochem. 14, 2257-2260 (2010).

250. Koyama, M. et al. The Mechanism of Porous Sm[sub 0.5]Sr[sub 0.5]CoO[sub 3] Cathodes Used in Solid Oxide Fuel Cells. J. Electrochem. Soc. 148, A795 (2001). 
